National Library

of Canada

Acquisitions and

Bibliographic Services Branch

395 Wellinglon Street

Ottawa. Onlario

KIA ON4
Bibliothèque nationale

du Canada

Direction des acquisitions el

des services bibliographiques

395, ne Wellington

Ottawa (Ontario)

KIA ON4
Yourtice vorretelerince

Outhe Notre retisinge
The quality of this microform is heavily dependent upon the quality of the original thesis submitted for microfilming. Every effort has been made to ensure the highest quality of reproduction possible.

If pages are missing, contact the university which granted the degree.

Some pages may have indistinct print especially if the original pages were typed with a poor typewriter ribbon or if the university sent us an inferior photocopy.

Reproduction in full or in part of this microform is governed by the Canadian Copyright Act, R.S.C. 1970, c. C-30, and subsequent amendments.
La qualité de cette microforme dépend grandement de la qualité de la thèse soumise au microfilmage. Nous avons tout fait pour assurer une qualité supérieure de reproduction.

S'il manque des pages, veuillez communiquer avec l'université qui a conféré le grade.

La qualité d'impression de certaines pages peut laisser à . désirer, surtout si les pages originales ont été dactylographiées à l'aide d'un ruban usé ou si l'université nous a fait parvenir une photocopie de qualité inférieure.

La reproduction, même partielle, de cette microforme est soumise à la Loi canadienne sur le droit d'auteur, SRC 1970, c. C-30, et ses amendements subséquents. 


\title{
The Effect of Turbulence on the Collision Rates of Small Cloud Drops
}

\author{
Anna S. Koziol \\ Department of Atmospheric and Oceanic Sciences \\ McGill University \\ Montréal
}

March 1995

A Thesis submitted to the Faculty of Graduate Studies and Research in partial fulfillment of the requirements for the degree of the DOCTOR of PHILOSOPHY in Atmospheric and Oceanic Sciences 
National Library

of Canada

Acquisitions and

Bibliographic Services Branch

395 Wellington Street

Ottawa, Ontario

KIA ONA
Bibliothèque nationale

du Canada

Direction des acquisitions et des services bibliographiques

395, ne Weilington

Otawa (Ontario)

KIA ON4
Your fite Volie rolference

Our five Notre rellerence
THE AUTHOR HAS GRANTED AN IRREVOCABLE NON-EXCLUSIVE LICENCE ALLOWING THE NATIONAL LIBRARY OF CANADA TO

REPRODUCE, LOAN, DISTRIBUTE OR SELL COPIES OF HIS/HER THESIS BY ANY MEANS AND IN ANY FORM OR FORMAT, MAKING THIS THESIS AVAILABLE TO INTERESTED PERSONS.
L'AUTEUR A ACCORDE UNE LICENCE IRREVOCABLE ET NON EXCLUSIVE PERMETTANT A LA BIBLIOTHEQUE NATIONALE DU CANADA DE REPRODUIRE, PRETER, DISTRIBUER OU VENDRE DES COPIES DE SA THESE DE QUELQUE MANIERE ET SOUS QUELQUE FORME QUE CE SOIT POUR METTRE DES EXEMPLAIRES DE CETTE THESE A LA DISPOSITION DES PERSONNE INTERESSEES.

L'AUTEUR CONSERVE LA PROPRIETE DU DROIT D'AUTEUR QUI PROTEGE SA THESE. NI LA THESE NI DES EXTRAITS SUBSTANTIELS DE CELLECI NE DOIVENT ETRE IMPRIMES OU AUTREMENT REPRODUITS SANS SON AUTORISATION.

ISBN $\quad 0-612-05734-8$ 


\section{To my late Father and B. G.}




\section{$\Delta_{\text {cknowledgements }}$}

Despite numerous obstacles or perhaps because of them I had a marvelous time while working on the subject of this thesis. However, it would be impossibly to conquer all the adverse currents which I encountered during iny research without. wise advice and help of many people.: Prof. Henry Leighton, my thesis supervisor is one of them. During years, I came to appreciate his unbiased physical intuition which saved me from many blunders. An invaluable scientific advice on a continuous basis in the areas of low Reynolds number flows and turbulence was given to me respectively by Prof. Raymond G. Cox from the Department of Civil Engineering and Applied Mechanics, and by Prof. Tom Warn from the Department of Atmospheric and Oceanic Sciences. Special thanks to Prof. David J. Jeffrey from the Department; of Applied Mathematics at the University of Western Ontario who helped me with the method of calculating the scalar resistance function coefficients and also provided me with a FORTRAN code to perform this task. Prof. Isztar Zawadzki from the Department of Atmospheric and Oceanic Sciences is gratefully acknowledged for many informal scientific chats from the very onset of this research as well as for enabling me to access the IDL graphics package which was used in this thesis.

The Atmospleric Environment Service, a nurturing mother of Canadian meteorologists is gratefully acknowledged for facilitating computer time on SX-3 NEC supercomputer. The help of Dr. Janusz Pudykiewicz from the AES who supported the use SX-3 NEC for my research is particularly appreciated. 


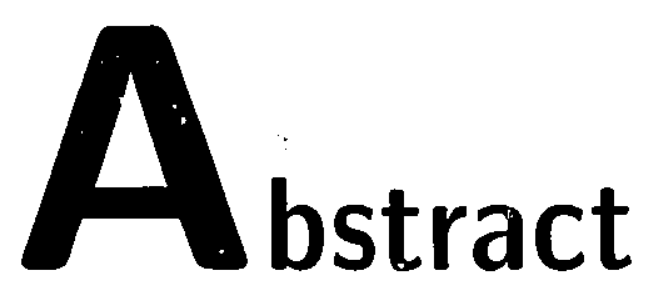

The role of turbulence in the process of collision and coalescencr of small cloud droplets is still an outstanding problem in the area of cloud physics. In particular, the growth of droplets in the radius range from 10 to $15 \mu \mathrm{m}$ is not well understocd. The present research has been motivated by the curiosity whether or not turbulence affects the growth rate of such small drops.

We developed a method to calculate collision rates of small hydrodynamically interacting drops embedded in an external flow field; we call it the flux method. Then, the method was tested for simple cases of laminar flows such as linear shear and a two-dimensional deformation field. The tests were designed not only to validate the Inethod but also to examine the mechanisms associated with the simplified types of external flows which may be equally important for real turbulent flows.

In order to obtain estimates of collision rates for turbulent flows, the flux method was used in conjunction with a probabilistic approach. Numerous simulations of trajectories of two hydrodynamically interacting droplets in a turbulent field were carried out. The ratio of the number of collisions to the total number of simulations gave the probability of collision for different relative positions of the drops. Because the Reynolds number of the flow around droplets (based on the drop radius and terminal velocity) is small, the trajectorics were calculated with the help of a model based on the linear Stokes hydrodynamics. Turbulence was modelled in the form 
of random Fourier modes with both the space and the time spectrum prescribed. Both spectra were characterized by Koimogorov scaling. The space spectrum was modelled in the inertial and dissipation subranges. On the basis of scale analysis, only small scale time variations were allowed, and, the so called Eulerian-Lagrangian time spectrum was applied.

The results show that most coliision rates increase muderately in a turbulent flow characterized by a rate of energy dissipation of the order of 1,10 , and $100 \mathrm{~cm}^{2}$ $\sec ^{-3}$. The estimated increase in collision efficiencies, however, is not uniform, and a rather complicated relation between the increase in the collision efficiency and the parameters-the drop radii, and the rate of energy dissipation-can be observed. 


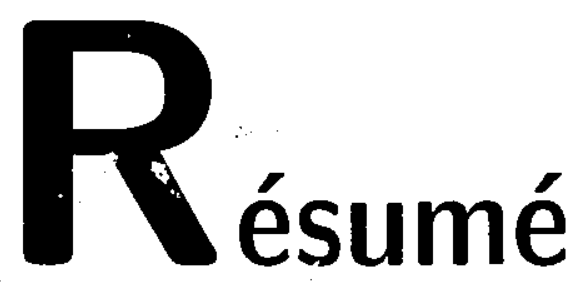

Le rôle de la turbulence dans le processus de collision et de coalescence des goutt.elettes nuageuses demeurc un problème important dans le domaine de la physique des nuages. En particulier, la croissance des petites gouttes de rayon variant de 10 à 15 $\mu \mathrm{m}$ reste mal comprise. La motivation prèmiere du présent travail est de déterminer si la turbulence a un effet sur la croissance des gouttelettes nuageuses.

Nous avons développé une méthode dite 'flux' pour calculer le taux de collisions de gouttelettes qui interagissent hydrodynamiquement dans un écoulement externe. La méthode fut testée pour des écoulements laminaires simples tels qu'un cisaillement linéaire et un écoulement bi-dimensionnel déformant. Le but de ces tests fut non seulement de valider la méthode mais aussi d'examiner les mécanismes de collisions dans les écoulements simples, lesquels peuvent être aussi importants que ceux règnant dans les écoulements turbulents réels.

Afin d'obtenir des estimations du taux de collision dans les écoulements turbulentes, la méthode flux fut utilisée dans un contexte probabiliste. Un grand nombre de simulations de trajectoires de deux gouttelettes interagissant hydrodynamiquement dans un écoulement turbulent a été réalisé. La probabilité de collision pour diverses positions relatives des gouttelettes fut calculée à partir du rapport entre le nombre de collisions et le nombre total de simulations. Dû au faible nombre de Reynolds de l'écoulement autour des gouttelettes (calculé à partir du rayon des gouttelettes et 
de leur vitesse terminale), les trajectoires furent calculées à l'aide d'un modìle basé sur l'approximation linéaire hydrodynamique de Stokes. L'ćcoulement turbulent f.tt. simulé en utilisant des modes de Fourier aléatoires prédéteiminés dans ic teirps et dans l'espace. Les modes ont été établis dans les échelles inertielle et dissipative. D'après une analyse d'échelle, seules les variations temporelles des petites échelles sont permises, et par conséquent, le spectre temporel Eulérien-lagrangien fut utilisé.

Les résultats montrent que la plupart des taux de collisions augmente modérément lorsque l'écoulement turbulent est caractérisé par un taux de dissipation de l'ordre de 1, 10 et $100 \mathrm{~cm}^{2} \mathrm{sec}^{-3}$. Toutefois. l'estimation de l'augmentation d'efficacité de collision est non-uniforme et on observe une relation plutôt compliquće entre l'augmentation d'efficacité de collision et les paramètres (rayon des goutteletities et: le taux de dissipation énergitique). 


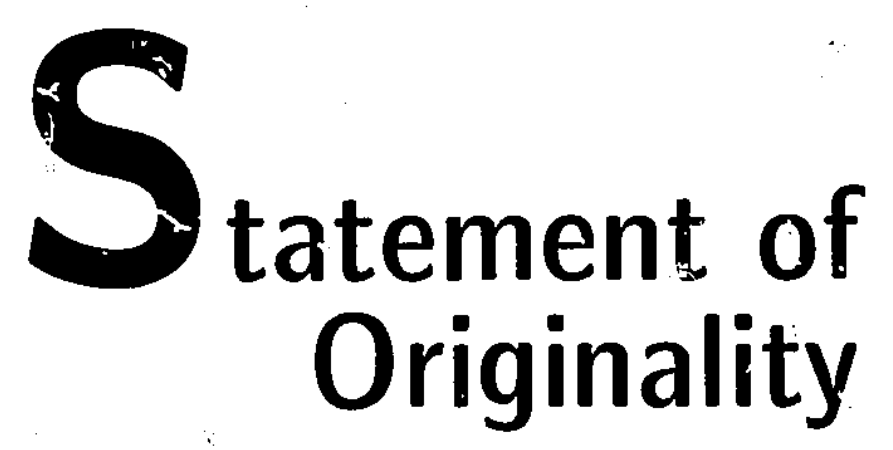

In this thesis, original research directed towards the understanding of the effect of turbulence cn collision rates of drops in developing cumulus clouds is presented.

The following are the original elements of the present research which were developed, and, later, successfully applied:

- The flux method was introduced as a tool with which to quantitatively determine the collision kernels and collision efficiencies.

- A numerical model to calculate trajectories of hydrodynamically interacting drops embedded in an external turbulent field was developed.

- The nnethod of random Fourier modes for turbulence modelling was modified, and, for the first time, used to model the motion field in a cloud. 


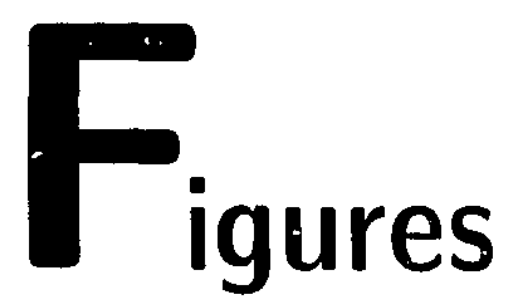

Fig. 4.1 The nondimensional wavenumber $(E)$ and frequency $\left(\chi_{i i}\right)$ spec-

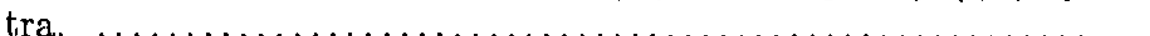

Fig. 5.1 Scalar resistance functions $X_{\alpha \beta}^{A}, Y_{\alpha \beta}^{A}$, and $Y_{\alpha \beta}^{B}$ vs. the nondimensional separation between drop surfaces $S /\left(r_{1}+r_{2}\right)$ for the radii ratio $\lambda=0.7$.

Fig. 5.2 Scalar resistance functions $X_{\alpha \beta}^{G}, Y_{\alpha \beta}^{G}$, and $Y_{\alpha \beta}^{H}$ vs. the nondimensional separation between drop surfaces $S /\left(r_{1}+r_{2}\right)$ for the radii ratio $\lambda=0.7$.

Fig. 5.3 Scalar resistance functions $X_{\alpha \beta}^{C}$, and $Y_{\alpha \beta}^{C}$ vs. the nondimensional separation between drop surfaces $S /\left(r_{1}+r_{2}\right)$ for the radii ratio $\lambda=0.7$.

Fig. 5.4a The drop coordinates, $x$ and $z$, the relative distance between drops, $\mathcal{D}$, the drop velocity components, $v_{x}$ and $v_{z}$, and the drop angular velocity component, $\Omega_{y}$ vs. time for the laminar settling of 15 and $8 \mu \mathrm{m}$ drops. Numbers indicate radii. No collision occurs.

Fig. 5.4b The drop relative coordinates, $x^{1}-x^{2}$ and $z^{1}-z^{2}$, and the drop relative velocity components, $v_{x}^{1}-v_{x}^{2}$ and $v_{z}^{1}-v_{z}^{2}$ vs. time for the laminar settling of 15 and $8 \mu \mathrm{m}$ drops. No collision occurs. .....

Fig. 5.5a The drop coordinates, $x$ and $z$, the relative distance between drops, $D$, the drop velocity components, $v_{x}$ and $v_{z}$, and the drop angular velocity component, $\Omega_{y}$ vs. time for the laminar settling of 15 and $14 \mu \mathrm{m}$ drops. Numbers indicate radii. No collision occurs.

Fig. 5.5b The drop relative coordinates, $x^{1}-x^{2}$ and $z^{1}-z^{2}$, and the clrop relative velocity components, $v_{x}^{1}-v_{x}^{2}$ and $v_{z}^{1}-v_{z}^{2}$ vs. time for the laminar settling of 15 and $14 \mu \mathrm{m}$ drops. No collision occurs. .... 
Fig. 5.6a The drop coordinates, $x, y$, and $z$, and the drop velocity components, $v_{x}, v_{y}$, and $v_{z}$ vs. time for the turbulent settling of 15 and $8 \mu \mathrm{m}$ drops and the rate of energy dissipation $1 \mathrm{~cm}^{2} \mathrm{sec}^{-3}$. The initial position of the smaller drop: $R=0.03 \mathrm{~cm}$ and $\theta=2.25^{\circ}$. Numbers indicate radii. The calculation results in a collision. ....

Fig. 5.6b The drop angular velocity components, $\Omega_{x}, \Omega_{y}$, and $\Omega_{z}$, and the turbulent velocity components, $u_{x}, u_{y}$, and $u_{z}$ at drop coordinates vs. time for the turbulent, settling of 15 and $8 \mu \mathrm{m}$ drops and the rate of energy dissipation $1 \mathrm{~cm}^{2} \mathrm{sec}^{-3}$. The initial position of the smaller drop: $R=0.03 \mathrm{~cm}$ and $\theta=2.25^{\circ}$. Numbers indicate radii. The calculation results in a collision.

Fig. 5.6c The drop relative coordinates, $x^{1}-x^{2}, y^{1}-y^{2}$, and $z^{1}-z^{2}$, and the drop relative velocity components, $v_{x}^{1}-v_{x}^{2}, v_{y}^{1}-v_{y}^{2}$, and $v_{z}^{1}-v_{z}^{2}$ vs. time for the turbulent settling of 15 and $8 \mu \mathrm{m}$ drops and the rate of energy dissipation $1 \mathrm{~cm}^{2} \mathrm{sec}^{-3}$. The initial position of the smaller drop: $R=0.03 \mathrm{~cm}$ and $\theta=2.25^{\circ}$. The calculation results in a collision

Fig. 5.7a The drop coordinates, $x, y$, and $z$, and the drop velocity components, $v_{x}, v_{y}$, and $v_{z}$ vs. time for the turbulent settling of 15 and $14 \mu \mathrm{m}$ drops and the rate of energy dissipation $1 \mathrm{~cm}^{2} \mathrm{sec}^{-3}$. The initial position of the smaller drop: $R=0.03 \mathrm{~cm}$ and $\theta=2^{\circ}$. Numbers indicate radii. The calculation results in a collision. ....

Fig. 5.7b The drop angular velocity components, $\Omega_{x}, \Omega_{y}$, and $\Omega_{z}$, and the turbulent velocity components, $u_{x}, u_{y}$, and $u_{z}$ at drop coordinates vs. time for the turbulent settling of 15 and $14 \mu \mathrm{m}$ drops and the rate of energy dissipation $1 \mathrm{~cm}^{2} \mathrm{sec}^{-3}$. The initial position of the smaller drop: $R=0.03 \mathrm{~cm}$ and $\theta=2^{\circ}$. Numbers indicate radii. The calculation results in a collision.

Fig. 5.7c The drop relative coordinates, $x^{1}-x^{2}, y^{1}-y^{2}$, and $z^{1}-z^{2}$, and the drop relative velocity components, $v_{x}^{1}-v_{x}^{2}, v_{y}^{1}-v_{y}^{2}$, and $v_{z}^{1}-v_{z}^{2}$ vs. time for the turbulent settling of 15 and $14 \mu \mathrm{m}$ drops and the rate of energy dissipation $1 \mathrm{~cm}^{2} \mathrm{sec}^{-3}$. The initial position of the smaller drop: $R=0.03 \mathrm{~cm}$ and $\theta=2^{\circ}$. The calculation results in a collision.

Fig. 5.8a The drop coordinates, $x, y$, and $z$, and the drop velocity components, $v_{x}, v_{y}$, and $v_{z}$ vs. time for the turbulent settling of 15 and $8 \mu \mathrm{m}$ drops and the rate of energy dissipation $10 \mathrm{~cm}^{2} \mathrm{sec}^{-3}$. The initial position of the smaller drop: $R=0.03 \mathrm{~cm}$ and $\theta=1^{\circ}$. Numbers indicate radii. The calculation results in a collision. .... 
Fig. 5.8b The drop angular velocity components, $\Omega_{x}, \Omega_{y}$, and $\Omega_{z}$, and the turbulent velocity components, $u_{x}, u_{y}$, and $u_{z}$ at drop coordinates vs. time for the turbulent settling of 15 and $S \mu \mathrm{m}$ drops and the rate of energy dissipation $10 \mathrm{~cm}^{2} \mathrm{sec}^{-3}$. The initial position of the smaller drop: $R=0.03 \mathrm{~cm}$ and $\theta=1^{\circ}$. Numbers indicate radii. The calculation results in a collision.

Fig. 5.8c The drop relative coordinates, $x^{1}-x^{2}, y^{1}-y^{2}$, and $z^{1}-z^{2}$, and the drop relative velocity components, $v_{x}^{1}-v_{x}^{2}, v_{y}^{1}-v_{y}^{2}$, and $v_{z}^{1}-v_{z}^{2}$ vs. time for the turbulent settling of 15 and $8 \mu \mathrm{m}$ drops and the rate of energy dissipation $10 \mathrm{~cm}^{2} \mathrm{sec}^{-3}$. The initial position of the smaller drop: $R=0.03 \mathrm{~cm}$ and $\theta=1^{\circ}$. The calculation results in a collision.

Fig. 5.9a The drop coordinates, $x, y$, and $z$, and the drop velocity components, $v_{x}, v_{y}$, and $v_{z}$ vs. time for the turbulent settling of 15 and $14 \mu \mathrm{m}$ drops and the rate of energy dissipation $10 \mathrm{~cm}^{2} \mathrm{sec}^{-3}$. The initial position of the smaller drop: $R=0.03 \mathrm{~cm}$ and $\theta=5^{\circ}$. Numbers indicate radii. The calculation results in a collision. ....

Fig. 5.9b The drop angular velocity components, $\Omega_{x}, \Omega_{y}$, and $\Omega_{z}$, and the turbulent velocity components, $u_{x}, u_{y}$, and $u_{z}$ at drop coordinates vs. time for the turbulent settling of 15 and $14 \mu \mathrm{m}$ drops and the rate of energy dissipation $10 \mathrm{~cm}^{2} \mathrm{sec}^{-3}$. The initial position of the smaller drop: $R=0.03 \mathrm{~cm}$ and $\theta=5^{\circ}$. Numbers indicate radii. The calculation results in a collision.

Fig. 5.9c The drop relative coordinates, $x^{1}-x^{2}, y^{1}-y^{2}$, and $z^{1}-z^{2}$, and the drop relative velocity components, $v_{x}^{1}-v_{x}^{2}, v_{y}^{1}-v_{y}^{2}$, and $v_{z}^{1}-v_{z}^{2}$ vs. time for the turbulent settling of 15 and $14 \mu \mathrm{m}$ drops and the rate of energy dissipation $10 \mathrm{~cm}^{2} \mathrm{sec}^{-3}$. The initial position of the smaller drop: $R=0.03 \mathrm{~cm}$ and $\theta=5^{\circ}$. The calculation results in a collision.

Fig. 5.10a The drop coordinates, $x, y$, and $z$, and the drop velocity components, $v_{x}, v_{y}$, and $v_{z}$ vs. time for the turbulent settling of 15 and $8 \mu \mathrm{m}$ drops and the rate of energy dissipation $100 \mathrm{~cm}^{2} \mathrm{sec}^{-33}$. The initial position of the smaller drop: $R=0.03 \mathrm{~cm}$ and $\theta=5^{\circ}$. Numbers indicate radii. The calculation results in a collision. ....

Fig. 5.10b The drop angular velocity components, $\Omega_{x}, \Omega_{y}$, and $\Omega_{z}$, and the turbulent velocity components, $u_{x}, u_{y}$, and $u_{z}$ at drop coordinates vs. time for the turbulent settling of 15 and $8 \mu \mathrm{m}$ drops and the rate of energy dissipation $100 \mathrm{~cm}^{2} \mathrm{sec}^{-3}$. The initial position of the smaller drop: $R=0.03 \mathrm{~cm}$ and $\theta=5^{\circ}$. Numbers indicate radii. The calculation results in a collision. 
Fig. 5.10c The drop relative coordinates, $x^{1}-x^{2}, y^{1}-y^{2}$, and $z^{1}-z^{2}$, and the drop relative velocity components, $v_{x}^{1}-v_{x}^{2}, v_{y}^{1}-v_{y}^{2}$, and $v_{z}^{1}-v_{z}^{2}$ vs. time for the turbulent settling of 15 and $8 \mu \mathrm{m}$ drops and the rate of energy dissipation $100 \mathrm{~cm}^{2} \mathrm{sec}^{-3}$. The initial position of the smaller drop: $R=0.03 \mathrm{~cm}$ and $\theta=5^{\circ}$. The calculation results in a collision.

Fig. 5.11a The drop coordinates, $x, y$, and $z$, and the drop velocity components, $v_{x}, v_{y}$, and $v_{z}$ vs. time for the turbulent settling of 15 and $14 \mu \mathrm{m}$ drops and the rate of energy dissipation $100 \mathrm{~cm}^{2} \mathrm{sec}^{-3}$. The initial position of the smaller drop: $R=0.03 \mathrm{~cm}$ and $\theta=15^{\circ}$. Numbers indicate radii. The calculation results in a collision. ....

Fig. 5.11b The drop angular velocity components, $\Omega_{x}, \Omega_{y}$, and $\Omega_{z}$, and the turbulent velocity components, $u_{x}, u_{y}$, and $u_{z}$ at drop coordinates vs. time for the turbulent settling of 15 and $14 \mu \mathrm{m}$ drops and the rate of energy dissipation $100 \mathrm{~cm}^{2} \mathrm{sec}^{-3}$. The initial position of the smaller drop: $R=0.03 \mathrm{~cm}$ and $\theta=15^{\circ}$. Numbers indicate radii. The calculation results in a collision.

Fig. 5.11c The drop relative coordinates, $x^{1}-x^{2}, y^{1}-y^{2}$, and $z^{1}-z^{2}$, and the drop relative velocity components, $v_{x}^{1}-v_{x}^{2}, v_{y}^{1}-v_{y}^{2}$, and $v_{z}^{1}-v_{z}^{2}$ vs. time for the turbulent settling of 15 and $14 \mu \mathrm{m}$ drops and the rate of energy dissipation $100 \mathrm{~cm}^{2} \mathrm{sec}^{-3}$. The initial position of the smaller drop: $R=0.03 \mathrm{~cm}$ and $\theta=15^{\circ}$. The calculation results in a collision.

Fig. 6.1 Collision in a still fluid. Schematic representation of the grazing trajectory and the impact parameter $y$.

Fig. 6.2 Schematic view of the geometry for the method of collection volume.

Fig. 6.3 Schematic view of the geometry for the flux method.

Fig. 6.4 Schematic view of the geometry for the flux method. The laminar case

Fig. 7.1 Collision efficiencies in still fluid calculated with the Stokes approxinnation and with the Oseen approximation in the limit of zero Reynolds number.

Fig. 7.2 The flux method for the case of a laminar shear flow. .......... 104

Fig. 7.3 The flux method for the case of a two-dimensional deformation field. 
Fig. 8.1 Probability $P(\theta)$ and $P(\theta) \sin 2 \theta$ for the typical case of: a) a 'weak turbulence' case $\left(r_{1}=10 \mu \mathrm{m}, r_{2}=6.5 \mu \mathrm{m}, \epsilon=1 \mathrm{~cm}^{2} \mathrm{sec}^{-3}\right)$, and b) a 'strong turbulence' case $\left(r_{1}=10 \mu \mathrm{m}, r_{2}=8 \mu \mathrm{m}, \epsilon=100\right.$ $\left.\mathrm{cm}^{2} \mathrm{sec}^{-3}\right)$.

Fig. 8.2 Comparison of the average radial 'in' component of the drop relative velocity for turbulent and laminar cases: a) $r_{1}=10 \mu \mathrm{m}$, $r_{2}=9.5 \mu \mathrm{m}, \epsilon=100 \mathrm{~cm}^{2} \mathrm{sec}^{-3}$, b) $r_{1}=10 \mu \mathrm{m}, r_{2}=5 \mu \mathrm{m}, \epsilon=1$ $\mathrm{cm}^{2} \mathrm{sec}^{-3}$.

Fig. 8.3 Comparison of the r.m.s. relative horizontal displacements due to turbulence and hydrodynamic forces for two pair of drops: 10 and $9 \mu \mathrm{m}$, and 15 and $5 \mu \mathrm{m}$. Solid lines represent the displacements due to turbulence for the energy dissipation rates from 0.01 to 100 $\mathrm{cm}^{2} \mathrm{sec}^{-3}$. Dashed lines represent the displacements due to the hydrodynamic forces in the laminar still-air settling. ......... 11

Fig. 8.1 Comparison of the laminar collision efficiencies with the turbulent collision efficiencies for the rate of energy dissipation equal to 0.01 $\mathrm{cm}^{2} \mathrm{sec}^{-3}$

Fig. 8.5 Schematic sketch for error calculation.

Fig. 9.1 Collision efficiencies for turbulent flows with different rates of energy dissipation $\left(\epsilon=100,10,1 \mathrm{~cm}^{2} \mathrm{sec}^{-3}\right)$, and for laminar flow. ..

Fig. 9.2 Comparison of the collision efficiencies for different strengths of turbulence with the laminar collision efficiencies for $10 \mu \mathrm{m}$ collector drops.

Fig. 9.3 Comparison of the collision efficiencies for different strengths of turbulence with the laminar collision efficiencies for $15 \mu \mathrm{m}$ collector drops.

Fig. 9.4 Comparison of the collision efficiencies for different, strengths of turbulence with the laminar collision efficiencies for $20 \mu \mathrm{m}$ collector drops.

Fig. 9.5 Comparison of the two-drop length scales for different drops (solid lines) with the Kolmogorov length scales for different strengths of turbulence (dashed lines).

Fig. 9.6 Comparison of the two-drop time scales for different drops (solid lines) with the Kolmogorov time scales for different strengths of turbulence (dashed lines), and the relaxation times for different collector drops (dotted lines). 
Fig. 9.7 Comparison of the terminal velocity differences for different pairs of drops (solid lines) with the Kolmogorov velocity scales for different strengths of turbulence (dashed lines).

Fig. 9.8 Probability $P(\theta)$ and $P(\theta) \sin 2 \theta$ illustrating the small perturbation mechanism: $\left(r_{1}=20 \mu \mathrm{m}, r_{2}=12 \mu \mathrm{m}, \epsilon=1 \mathrm{~cm}^{2} \mathrm{sec}^{-3}\right)$, and $\left(r_{1}=10 \mu \mathrm{m}, r_{2}=6.5 \mu \mathrm{m}, \epsilon=1 \mathrm{~cm}^{2} \mathrm{sec}^{-3}\right)$; increase of the collision efficiency. Dotted lines represent the laminar cases. . . .. 136

Fig. 9.9 Collision efficiencies for very weak turbulent flows with different rates of energy dissipation $\left(\epsilon=0.1,0.01 \mathrm{~cm}^{2} \mathrm{sec}^{-3}\right) . \ldots \ldots \ldots 137$

Fig. 9.10 Comparison of the collision efficiencies for different strengths of weak turbulence with the laminar collision efficiencies for the 10 $\mu \mathrm{m}$ collector drop. (The laminar collision efficiencies were obtained for the same drop separations as those for turbulent flows.) . . 138

Fig. 9.11 Comparison of the collision efficiencies for different strengths of weak turbulence with the laminar collision efficiencies for the 15 $\mu \mathrm{m}$ collector drop. (The laminar collision efficiencies were obtained for the same drop separations as those for turbulent flows.) .. 138

Fig. 9.12 Probability $P(\theta)$ and $P(\theta) \sin 2 \theta$ illustrating 'large' scale mixing: $\left(r_{1}=10 \mu \mathrm{m}, r_{2}=3.5 \mu \mathrm{m}, \epsilon=100 \mathrm{~cm}^{2} \mathrm{sec}^{-3}\right)$, and $\left(r_{1}=20 \mu \mathrm{m}\right.$, $\left.r_{2}=19 \mu \mathrm{m}, \epsilon=10 \mathrm{~cm}^{2} \mathrm{sec}^{-3}\right)$. Dotted lines represent angles $\theta$ corresponding to the grazing trajectories.

Fig. 9.13 Collision kernels for turbulent flows with different rates of energy dissipation $\left(\epsilon=100,10,1 \mathrm{~cm}^{2} \mathrm{sec}^{-3}\right)$, and for laminar flow. Dotted lines mark the extension of the collision kernels for identical drops. 


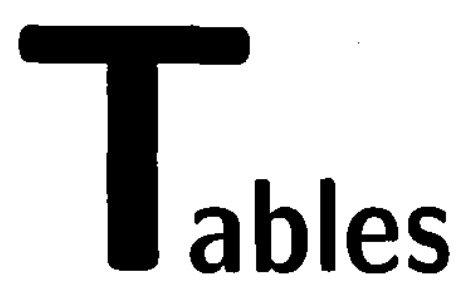

TABLE 2.1 Characteristic Scales of a Single Drop $\ldots \ldots \ldots \ldots \ldots \ldots \ldots \ldots .23$

TABLE 2.2 Characteristic Scales of Two-Drop Interactions ............. 23

TABLE 2.3 Characteristic Scales of Turbulence $\ldots \ldots \ldots \ldots \ldots \ldots \ldots \ldots . \ldots \ldots$

TABLE 7.1 Collision Kernels for Two-Dimensional Deformation Field ...... 109

TABLE 8.1 The Dependence of the Collision Efficiency on the Initial Dis-

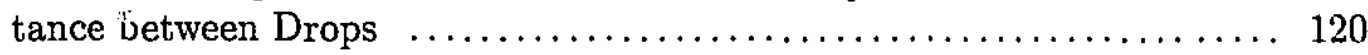

TABLE 8.2 The Dependence of the Collision Efficiency on Turbulence Truncation $\ldots \ldots \ldots \ldots \ldots \ldots \ldots \ldots \ldots \ldots \ldots \ldots \ldots \ldots \ldots \ldots \ldots, 121$

TABLE 9.1 Turbuleit Collision Efficiencies for Different Radii and Different Rates of Energy Dissipation ......................... 127

TABLE 9.2 Turbulent Collision Efficiencies for Very Weak Turbulence ...... 139 


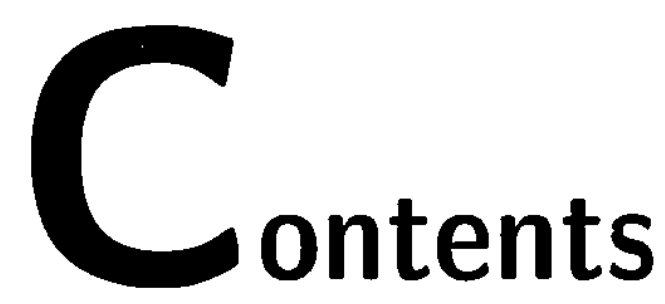

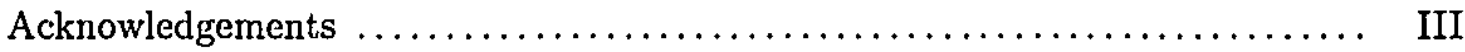

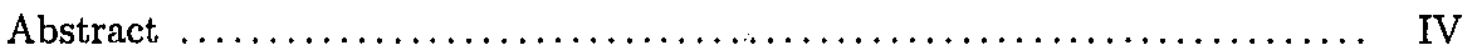

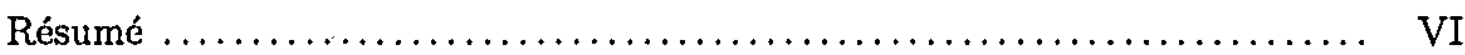

Statement of Originality $\ldots \ldots \ldots \ldots \ldots \ldots \ldots \ldots \ldots \ldots \ldots \ldots \ldots \ldots \ldots \ldots \ldots$ VIII

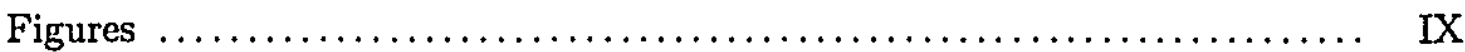

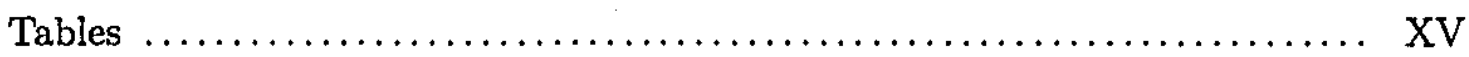

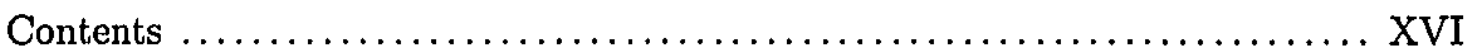

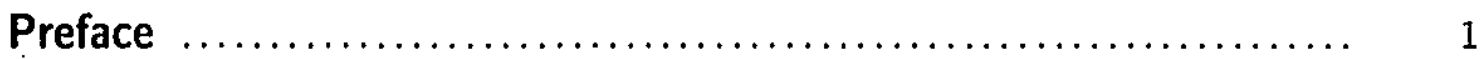

1 Turbulence and Collisions. A Critical Review of Literature ...... 4

1.1 The Effect of Inertia-Early Research $\ldots \ldots \ldots \ldots \ldots \ldots \ldots \ldots \ldots, 4$

1.2 The Rate of Energy Dissipation-A Fundamental Parameter ...... 6

1.3 The Diffusion Equation $\ldots \ldots \ldots \ldots \ldots \ldots \ldots \ldots \ldots \ldots \ldots \ldots, 10$

1.4 Modelling Drop Trajectories $\ldots \ldots \ldots \ldots \ldots \ldots \ldots \ldots \ldots \ldots \ldots . \ldots \ldots \ldots \ldots$

1.5 Experimental Evidence $\ldots \ldots \ldots \ldots \ldots \ldots \ldots \ldots \ldots \ldots \ldots, \quad 17$

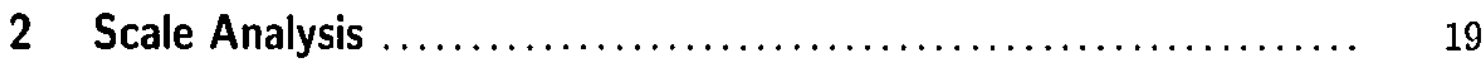

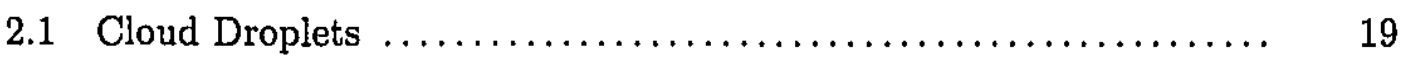

2.2 Cloud Turbulence $\ldots \ldots \ldots \ldots \ldots \ldots \ldots \ldots \ldots \ldots \ldots \ldots \ldots \ldots \ldots, 24$

3 Microhydrodynamics of Cloud Droplets .................. 26

3.1 The Low-Reynolds Number Approach $\ldots \ldots \ldots \ldots \ldots \ldots \ldots \ldots \ldots .26$

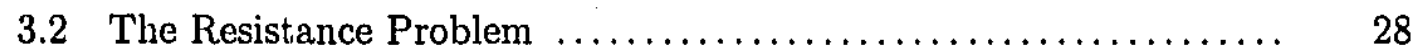

3.3 Solutions of the Stokes Equation $\ldots \ldots \ldots \ldots \ldots \ldots \ldots \ldots \ldots \ldots, \quad 30$

3.3.1 Method of Bispherical Coordinates ................. 31

3.3.1.1 Axisymmetric Problem ..................... 32

3.3.1.2 Problems with a Plane of Symmetry .......... 32 
3.3.2 Method of Reflections $\ldots \ldots \ldots \ldots \ldots \ldots \ldots \ldots \ldots \ldots \ldots, 33$

3.3.3 Asymptotic Methods ........................... 34

3.4 Molecular Effects ................................... 35

4 Modelling of the Turbulent Velocity Field ................. 37

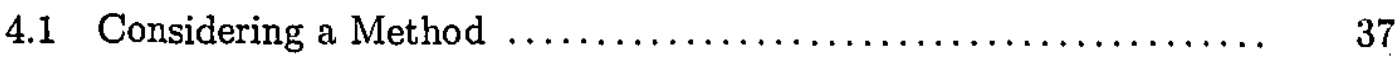

4.2 Some Aspects of Statistical Description of Turbulence .......... 39

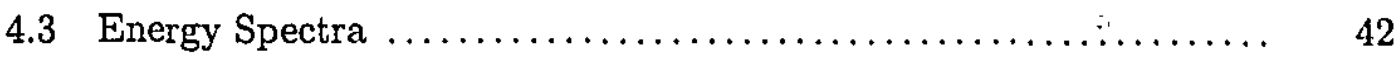

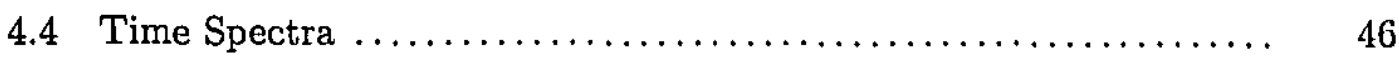

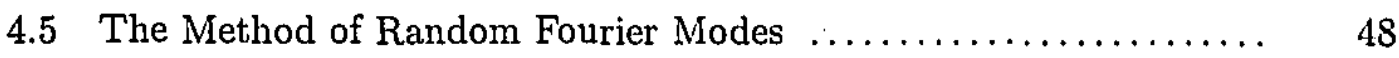

4.6 Realizations of the Turbulent Field $\ldots \ldots \ldots \ldots \ldots \ldots \ldots \ldots \ldots \ldots \ldots \ldots \ldots$

5 Modelling Drop Trajectories .......................... 55

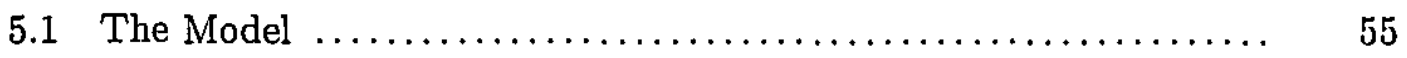

5.1 .1 The Scalar Resistance Functions ................ 56

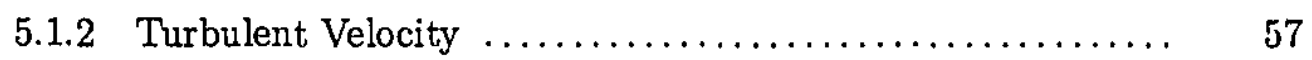

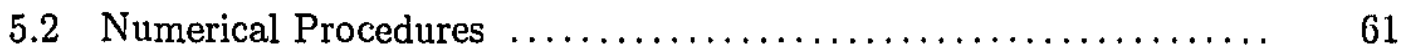

5.3 Trajectory Simulations $\ldots \ldots \ldots \ldots \ldots \ldots \ldots \ldots \ldots \ldots \ldots \ldots \ldots \ldots \ldots$

6 Collision Kernels and Collision Efficiencies ................... 88

6.1 The Stochastic Coalescence Equation $\ldots \ldots \ldots \ldots \ldots \ldots \ldots \ldots . \ldots \ldots$

6.2 Turbulent Kernels and Collision Efficiencies ................ 91

6.2.1 The Method of Collection Volume ................ 91

6.2 .2 The Flux Method ........................ 92

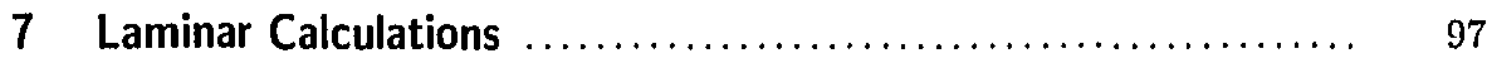

7.1 An Overview of Methods of Calculating Collision Efficiencies

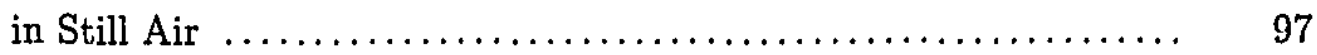

7.1.1 The Superposition of Flow Fields ............... 97

7.1.2 Direct Calculation of Hydrodynamic Forces ........... 98

7.1.3 The Choice of the Hydrodynamic Representation ........ 100

7.2 The Case of a linear Shear $\ldots \ldots \ldots \ldots \ldots \ldots \ldots \ldots \ldots \ldots \ldots \ldots . \ldots \ldots$

7.3 The Case of a Two-Dimensional Deformation Field ............ 105

7.4 Collision Efficiency for Almost Equal Drops ............... 107 


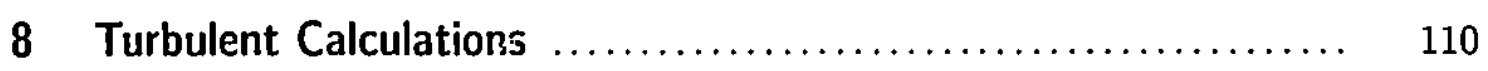

8.1 The Operational Details of the Model ................... 110

8.2 Calculation of the Average 'in' Component of the Radial

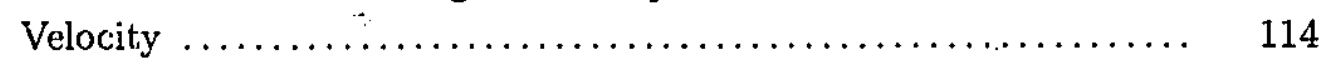

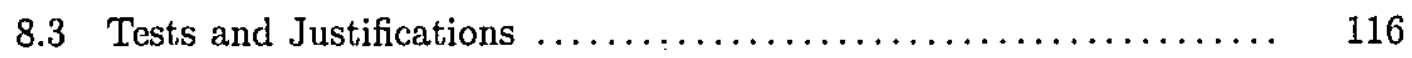

8.3.1 The Dependence of the Collision Efficiency on the Initial Distance between Drops ....................... 116

8.3.2 The Dependence of the Collision Efficiency on the Truncation of the Turbülent Field ................. 120

8.3.3 The no-Effect Limit of the Rate of Energy Dissipation .... 121

8.4 Error Analysis $\ldots \ldots \ldots \ldots \ldots \ldots \ldots \ldots \ldots \ldots \ldots \ldots \ldots \ldots, 122$

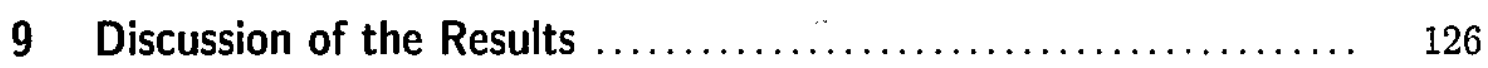

9.1 Presentation of the Basic Results $\ldots \ldots \ldots \ldots \ldots \ldots \ldots \ldots \ldots . \ldots \ldots$

9.2 Results from the Point of View of Scale Analysis ............. 133

9.2.1 Small Perturbations to Gravitational Laminar

Settling ................................... 135

9.2 .2 Turbulent Mixing $\ldots \ldots \ldots \ldots \ldots \ldots \ldots \ldots \ldots \ldots \ldots \ldots . \quad 140$

9.3 Collision Kernels and the Extension of the Results for Identical Drops .................................. 142

9.4 Suggestions for the Improvement of the Results $\ldots \ldots \ldots \ldots \ldots \ldots . \quad 144$

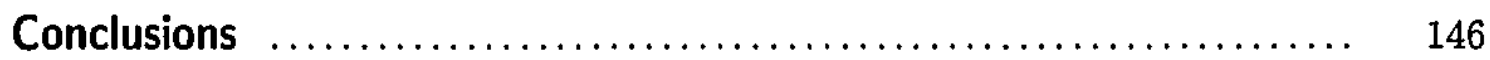

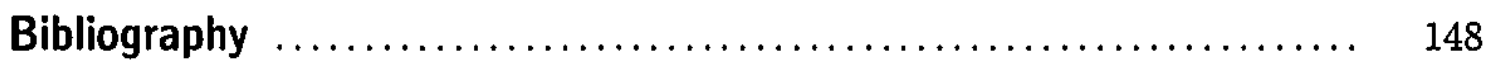




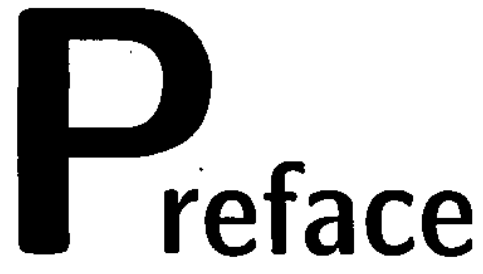

How rain forms-a basic part of the education of a meteorology student, yet, surprisingly enough, a subject of serious research of only the past. 50 years; even more surprisingly, a subject still not well understood. Although, precipitation is governed by large scale weather systems, microphysics of clouds plays a significant role, and as such needs to be properly addressed. The process of rain formation under consideration here-collision-coalescence-is one of the two recognized mechanisms of rain formation. The initial growth of cloud droplets is entirely due to condensation. Condensation produces droplets as big as $10 \mu \mathrm{m}$ in radius but above that size condensational growth rates become so slow as to be ineffective, and other processes must take over in order to generate large rain-size drops. One possibility is that the next stage of formation of precipitation involves the ice phase. Water vapour which is saturated with respect to liquid water become supersaturated with respect to icethus diffusional growth of ice crystals is vigorous when ice crystals formed in such a way encounter higher temperatures, they melt, and fall down in the form of rain. This is the, so called, Wegener-Bergeron-Findeisen process. The alternative scenario does not involve the solid phase. Due to their different terminal velocities, drops of different sizes will collide and possibly coalesce which is predictably termed as collision-coalescence.† Metaphorically speaking; 'collision-coalescence is a 'Cinderella' among the rain formation mechanisms, since for many years the Wegener-Bergeron-

$\dagger$ In mechanics of aerosols the analogous process is called coagulation while in the early years metcorologists tend to use the word coalescence. We prefer the term collision-coalescence as it reflects the double nature of the effect. 
Findeisen process was the principal concern of meteorologists. However, the idea that collisions between drops of different sizes are responsible for the production of large, rain-size drops, was introduced for the first time in the eighteenth centuryindependently, in 1715 by Barlow, and in 1739 by Musschenbroek [Pruppacher \& Klett, 1980.] Later, Reynolds [1876] even predicted that droplets above a certain size may grow more effectively by collisions than by diffusion. The concept was then abandoned for half of a century. Finally, in 1941, Simpson in his presidential address to the Royal Meteorological Society started a new era by bringing the forgotten idea of collisional growth to the attention of the meteorological community. (This move was strongly supported by the observations by pilots flying over India.) Presently, the observational evidence suggests that the collision-coalescence process occurs not only in clouds with cloud-top temperatures above zero Celsius but also in clouds where supercooled droplets are present, at temperatures of the order of $-20 \mathrm{C}$ (often referred to as non-freezing clouds) [Rogers \& Yau, 1989.]

There are many aspects of the collision-coalescence process which are not well explained. In particular, little is known about coalescence. (Most of the research has been geared toward the much easier to model process of collision.) Experiments show that collisions of drops do not always result in a permanent union-a thin layer of air between drops preventing coalescence. For larger drops, the coalescence efficiency may be then lower than unity [Ochs et al., 1986] which is assumed in most applications. There is no consensus about the 'easier' collision part. Droplets smaller than $10 \mu \mathrm{m}$ radii grow efficiently through diffusion, while, according to the laminar theory, droplets larger than $20 \mu \mathrm{m}$ radii grow efficiently through collisions. Clearly, there is a size gap where drops do not grow vigorously by either of these processes. Also, for the same range of radii, the results of empirical 'in vitro' measurements of collision rates do not converge with theoretical results [Jonas \& Goldsmith, 1972] 
(this is except for settling in a still fluid.) The fact that there are links missing somewhere has been known for some time. In particular, in 1939, while Europe was facing World War II, in the quiet Mt. Washington Observatory in New Hampshire, Arenberg [1939] was writing: "Turbulence as the major factor in the growth of cloud drops." Whether that statement is correct or not has been under investigation ever since. Incidentally, this is also the subject, of this dissertation.

The very basic question posed at the beginning of the research presented here was that concerning methodology-how to estimate the effect of turbulence, and, in particular, how to compare it with that of the laminar approximation. Upon consideration, the estimate of collision rates for pairs of drops by direct examination of their trajectories was found to be the most viable method. The unavoidable tasks brought by the above approach were to obtain an instantaneous turbulent velocity field, and to calculate trajectories of two hydrodynamically interacting drops. By introducing a concept of turbulent collision efficiency (as in de Almeida [1975]), the comparison between collision efficiencies in laminar and turbulent flows as well as a determination of the role of turbulence were possible. Certainly, the results do not, answer once and for all the question of how important is turbulence as a factor in the growth of cloud drops. Hopefully, however, they will serve as a step for the future development of the theories of rain formation. 


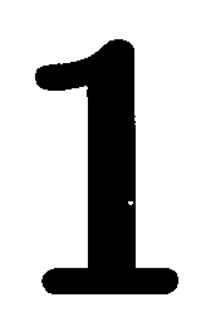

\section{Turbulence and Collisions A Critical Review of Literature}

A wide variety of approaches have been applied in order to find the possible role of turbulence in the process of collision-coalescence. In this chapter, we present the major trends as well as emphasize the results which are significant from the point of view of the present work.

\subsection{The Effect of Inertia-Early Research}

Most early work looked at the effect of drop inertia as the exclusive mechanism to enhance collisions in turbulent media. From a modern point of view, the, so called, inertial effect can be briefly defined [Yudine, 1959] as a property of particles having higher densities than that of the surrounding fluid to leave trajectories followed by fluid parcels. The noticeable effects of the above are: the lower velocity of the particle in comparison to the surrounding fluid as well as the retention of velocity correlations by particles for longer periods of time. Particles (or droplets in our case) of different sizes exhibit different 'degrees' of the inertial effect. In the works by Arenberg [1939], Gabilly [1949], and East \& Marshall [1954], the relative displacement of two drops due to the inertial effect was compared to the relative displacement due to gravity. 
While 'turbulence' in the two earlier papers was exclusively modelled as a sinusoidal motion, East \& Marshall applied a random velocity field. Although the actual results of East \& Marshall are of historical value now, it is worth presenting both the method and the results because they influenced future thinking.

In a one-dimensional, random ambient velocity field, the relative (vertical) velocity of drops, $w(t)$ has a Gaussian distribution. Because the motion of drops is caused by the random velocity, $u(t)$, the variance of the distribution, $w(t)$, must be solely determined by the random velocity field. Making use of the theory of stationary random functions, the variance of the relative velocity is

$$
\sigma_{w}^{2}=\int_{0}^{\infty}\left|\frac{w}{u}\right| G(\omega) d \omega
$$

where $G(\omega)$ is the spectral density function for the random variable $u(t)$ and

$$
\sigma_{u}^{2}=\int_{0}^{\infty} G(\omega) d \omega
$$

Various forms of $G(\omega)$ have been examined (e.g. Gaussian, uniform, delta, and Markoff distributions.) For most of them, a similar dependence of the variance of the drop relative velocity on the variance of the acceleration of the random field $\left(\sigma_{a}^{2}\right)$ was found

$$
\sigma_{w}^{2}=\sigma_{a}^{2}\left(\frac{m_{1}}{6 \pi \nu r_{1}}-\frac{m_{2}}{6 \pi \nu r_{2}}\right)^{2},
$$

where $r_{1}$ and $r_{2}$, and $m_{1}$ and $m_{2}$ are respectively the radii and the masses of the drops, while $\nu$ is the kinematic viscosity of air. The analogue of the above equation for gravitational coagulation in a still fluid is

$$
w=g\left(\frac{m_{1}}{6 \pi \nu r_{1}}-\frac{m_{2}}{6 \pi \nu r_{2}}\right) .
$$

East \& Marshall pushed the analogy even further, by calculating the relative volume swept by the collector drop as

$$
V=2 \pi\left(r_{1}+r_{2}\right)^{2} \int_{0}^{\infty} E(w) w P(w) d w
$$


where $E(w)$ is the collision efficiency which, in this particular situation, is a function of the relative velocity, $w$, and $P(w)$ is the probability density. Note that for the gravitational case

$$
V=\pi\left(r_{1}+r_{2}\right)^{2} E w
$$

The collision efficiencies $E$ and $E(w)$ were calculated according to Langmuir [1948]. East \& Marshall examined collision rates for the following situations: random motion with the r.m.s. value $\sigma_{a}$, equal to gravity, gravity alone, and random motion with gravity. Two major conclusions of their research were: 1 . The random motion alone enhances the collisions between droplets of small radius ratios as compared to the gravitational case. 2. Any fluctuating component parallel to gravity increases the collision rates. (Note that the fluctuating component which was assumed was of the order of gravity-hence the effect was significant.) The major criticism which East \& Marshall faced was that concerning the actual character of turbulence as opposed to their model of random motion. In particular, no spatial variation of the velocity was allowed [Saffman \& Turner, 1956]. This is a criticism rather difficult to refute. Further, treating hydrodynamic interactions in the same way as for the laminar case was not correct. Still, the qualitative character of the effect of inertia on collision efficiencies was captured [Saffman \& Turner, 1956].

\subsection{The Rate of Energy Dissipation-A Fundamental Parameter}

In order to enlighten the role of turbulence in the process of collisions between small cloud drops, a realistic model of turbulence was applied by Saffman \& Turner [1956]. The fundamental assumption of that research was the isotropic character of the turbulent field which is justified since only the smallest scales can affect collisions 
of small cloud droplets. They recognized that drops may collide due to two possible mechanisms: their different motions relative to the air and different motions with the air. While the first mechanism does not allow for collisions of drops of the same size, the second inherently has no such restrictions. The research delivered by Saffman \& Turner emphasized collisions between nearly identical drops, and, consequently, did not take the hydrodynamic interactions into account. (The experimental evidence available at that time [Manley \& Mason, 1952] suggested that the collection efficiency of almost identical drops is equal to unity.) Two analyses were performed, one concerning exclusively the collisions due to the motion with the air, and another including all three elements: motion with the air, and motion relative to the air duc to turbulence and due to gravity.

The collision rates due to the motion with the air were estimated by calculating the flux of drops through a sphere of radius equal to the sum of the drop radii, $r_{1}+r_{2}$. This flux was estimated as

$$
F=2 \pi\left(r_{1}+r_{2}\right)^{2} \overline{\left|w_{x}\right|}
$$

where $w_{x}$ is the relative radial velocity along the radius parallel to the $x$-axis (note that turbulence is isotropic). Since $r_{1}+r_{2}$ is small with comparison to the small eddies, and, further, using the result obtained by Taylor [1935], the following can be written

$$
\overline{\left|w_{x}\right|}=\left(r_{1}+r_{2}\right) \frac{\overline{\partial u_{x}}}{\partial x}=\left(r_{1}+r_{2}\right)\left(\frac{2 \epsilon}{15 \nu \pi}\right)^{\frac{1}{2}}
$$

where $u_{x}$ is the $x$-component of the turbulent velocity, and $\epsilon$ is the rate of energy dissipation. The collision rate is thus

$$
\mathcal{N}=n_{1} n_{2}\left(r_{1}+r_{2}\right)^{3}\left(\frac{8 \pi \epsilon}{15 \nu}\right)^{\frac{1}{2}}
$$

where $n_{1}$ and $n_{2}$ are the concentrations of drops. Clearly, the collision rates are dependent on radii as well as on the rate of energy dissipation. With the help of 
the 'predecessor' of the stochastic growth equation [Smoluchowski, 1917], Saffman \& Turner concluded that the significant growth of initially uniform droplets may occur for the rate of energy dissipation equal to $1000 \mathrm{~cm}^{2} \mathrm{sec}^{-3}$.

In the second approach, the path of East \& Marshall was roughly followed. From the results of molecular theory, for nonuniform gases in particular [Chapman \& Cowling, 1970], the collision rate is

$$
\mathcal{N}=\pi\left(r_{1}+r_{2}\right)^{2} n_{1} n_{2} \iiint w P(\mathbf{w}) d \mathbf{w}
$$

where $P(\mathbf{w})$ is the probability distribution function of the relative velocity. Saffman \& Turner chose the relatively simple form of a three-dimensional Gaussian distribution. In order to find the variance of the distribution, the velocities of the drops were decomposed into the motion with the air, $\mathbf{u}$, and relative to the air, $\mathbf{q}$

$$
\mathrm{v}_{1}=\mathrm{u}_{1}+\mathrm{q}_{1} \quad \mathrm{v}_{2}=\mathrm{u}_{2}+\mathrm{q}_{2}
$$

Clearly, the variance of the relative droplet velocity $w$ is

$$
\operatorname{var}(\mathbf{w})=\operatorname{var}\left(\mathbf{v}_{2}-\mathbf{v}_{1}\right)=\operatorname{var}\left(\mathbf{u}_{2}-\mathbf{u}_{1}\right)+\operatorname{var}\left(\mathbf{q}_{2}-\mathbf{q}_{1}\right)
$$

In order to evaluate the variance associated with the motion of the air, the properties of turbulence were exploited once again

$$
\operatorname{var}\left(\mathbf{u}_{2}-\mathbf{u}_{2}\right)=\left(r_{1}+r_{2}\right)^{2} \overline{\left(\frac{\partial \mathbf{u}}{\partial x}\right)^{2}}=5\left(r_{1}+r_{2}\right)^{2} \overline{\left(\frac{\partial u_{x}}{\partial x}\right)^{2}}=\frac{1}{3}\left(r_{1}+r_{2}\right)^{2} \frac{\epsilon}{\nu} .
$$

$=$ The variance of the velocities relative to the air was estimated by averaging the drop equations of motion in the form

$$
\frac{d \mathbf{v}_{1}}{d t}=-\frac{9 \mu}{2 r_{1}^{2} \rho_{0}}\left(\mathbf{v}_{1}-\mathbf{u}_{1}\right)+\frac{\rho}{\rho_{0}} \frac{d \mathbf{u}_{1}}{d t}+\left(\frac{\rho}{\rho_{0}}-1\right) \mathbf{g}
$$

where $\mathbf{g}$ is the acceleration due to gravity, and $\rho$ and $\rho_{0}$ are the air and drop densities respectively. (Analogous equation holds for the second drop.) Without going into the 
details of the calculation, let us look at the final expression for the collision rate

$$
\begin{aligned}
& \mathcal{N}=2(2 \pi)^{\frac{1}{2}}\left(r_{1}+r_{2}\right)^{2} n_{1} n_{2}[\underbrace{\left(1-\frac{\rho}{\rho_{0}}\right)^{2} \frac{9 \mu}{2 \rho_{0}}\left(\frac{1}{r_{1}^{2}}-\frac{1}{r_{2}^{2}}\right)^{2} \overline{\left(\frac{D u_{x}}{D t}\right)^{2}}}_{\text {Notion relutive to the air due to turbulence }}+ \\
&\underbrace{\frac{1}{3}\left(1-\frac{\rho}{\rho_{0}}\right)^{2} \frac{9 \mu}{2 \rho_{0}}\left(\frac{1}{r_{1}^{2}}-\frac{1}{r_{2}^{2}}\right)^{2} g^{2}}_{\text {Motion relative to the air due to gravity }}+\underbrace{\frac{1}{9}\left(r_{1}+r_{2}\right)^{2} \frac{\epsilon}{\nu}}_{\text {Motion with the nir }}],
\end{aligned}
$$

where $\frac{D u_{x}}{D t}$ is the Lagrangian time derivative which was assumed to be equal to the Eulerian time derivative $\frac{d u_{x}}{d t}$. Saffman \& Turner concluded the following. In heterogeneous clouds, the collision rates due to the motion with the air are higher than those due to the motion relative to the air (except for very small droplets $1-10 \mu \mathrm{m}$ in radius depending on the strength of turbulence.) The collision rates due to the motion relative to the air will increase significantly for the r.m.s. accelerations of turbulence of the order of gravity which corresponds to the rate of energy dissipation of approximately $2000 \mathrm{~cm}^{2} \mathrm{sec}^{-3}$.

In the paper by Saffman \& Turner, a realistic (up to date in 1956) model of turbulence was used. They assumed a homogeneous and isotropic turbulent field (a justified assumption on such a small scale), and pointed out that the rate of energy dissipation is the only parameter of turbulence which is crucial for the process under consideration. In other words, the effect of turbulence on the collisional growth of cloud drops is solely governed by Kolmogorov scaling. Despite forty years of research, the fundamentals of the theory of homogeneous turbulence have not been discredited. However, the entire approach of Saffman \& Turner is geared toward finding the effect. of an average turbulent field on collisions. As was pointed out by Narashima [1990], the averaged turbulent field (as governed, for example, by the Reynolds equations) is just one of many possible realizations, and, consequently, may not represent the real field. However, the results obtained by Saffman \& Turner should be treated as a 'first order approximation' on which to build more refined methods by considering not, 
average but instantaneous fields. The major shortcoming of this otherwise magnificent paper is not including the hydrodynamic forces, but, this would not be an easy task in the formulation applied by Saffman \& Turner. Finally, the use of the Gaussian distribution of the relative drop velocity seems to be somewhat arbitrary and may have affected the quantitative results.

\subsection{The Diffusion Equation}

The analogy between molecular and turbulent diffusion was pointed out for the first time in the famous paper by Taylor [1921]: "Diffusion by Continuous Movements". Since then, the idea has been widely employed to describe the ability of turbulent motion to mix or transport momentum, kinetic energy, heat, particles, etc. (see for example the text by Tennekes \& Lumley [1980].) In particular, in the area of the mechanics of aerosols, the diffusion equation has been extended to turbulent motion (various applications can be found in the text by Fuchs [1964].) From the mechanics of aerosols, the diffusion equation was then introduced to cloud physics. Smirnov [1968], delivered a through analysis of all the possible factors involved in the process of coagulation of cloud droplets. (We purposely switch to the term coagulation because this is the word preferred by scientists in the field of mechanics of aerosols.) Diffusion onto a sphere streaming with the velocity $\mathbf{v}$ is not governed by Fick's second law

$$
\frac{\partial n}{\partial t}=\nabla \cdot \nabla D n
$$

(where $n$ is the concentration of particles, and $D$ is the coefficient of diffusion) but instead, it, satisfies the Smoluchowski equation [1916]

$$
\frac{\partial n}{\partial t}=\nabla \cdot(\nabla D n-\mathbf{v} n)
$$

In fact Smirnov used both of the above equations to examine a wide range of processes: 
molecular diffusion, molecular diffusion with gravity, turbulent diffusion, molecular and turbulent diffusion, molecular and turbulent diffusion with gravity, molecular diffusion with electrostatic forces, turbulent diffusion with electrostatic forces, and turbulent and molecular diffusion with electrostatic forces. The resulis were obtained for collector drops in the range 1-15 $\mu \mathrm{m}$. Smirnov applied the solution of a steadystate Smoluchowski equation which was derived by Frisch [1954]. (Frisch calculated the flux of aerosol particles onto a streaming sphere. This flux, divided by the concentration of the collector drops gives the collision rate.) For the case of molecular diffusion with gravity, Smirnov used a convenient form of the flux in terms of the nondimensional Reynolds, Knudsen, Stokes, and Péclet numbers. In order to represent turbulence in these nondimensional numbers, the appropriate values of the time, space, and velocity scales were applied. (These depend on the kincmatic viscosity and the rate of energy dissipation.) Also, it was recognized that the drop velocity can be represented by a superposition of the 'inertial' (motion relative to the air), and 'diffusional' (motion with the air) components. Hydrodynamic interactions were not. taken into account.

A brief summary of the results follows. The effect of turbulence (for the rate of energy dissipation equal to $100 \mathrm{~cm}^{2} \mathrm{sec}^{-3}$ ) was found to be significant for both molecular-turbulent and molecular-turbulent-gravitational cases compared with molecular and molecular-gravitational cases respectively. Also, comparisons were made between the molecular and molecular-gravitational cases-the associated increase of collision efficiencies was tenfold. The model clearly demonstrated that turbulence and gravity reinforce each other leading to highest collision rates. Finally, no significant effect of electrostatic forces in the cases including both turbulence and electrostatic forces was observed.

We start the critical discussion about the approach presented by Smirnov with 
the assessment of the assumption of constant diffusivity. The assumption of constant diffusivity, which is a correct approximation for molecular diffusion does not work equally well for turbulent diffusion. This is because the time scales of interest are usually not much longer than the Lagrangian time scale characterizing turbulence. Still, it is reasonable to accept the above as a crude approximation (see Tennekes \& Lumley [1980], p. 226.) Further, Smirnov used the solution of (1.17), where the collector drop is assumed to stream with a constant velocity (may be not quite appropriate for $\epsilon=100 \mathrm{~cm}^{2} \mathrm{sec}^{-3}$ ), and he substituted arbitrarily the 'laminar' parameters by 'turbulent' ones. It is difficult to estimate the possible errors of the above steps.

For all turbulent cases, the inertial effect was neglected because the droplets considered were small. The error due to neglecting inertia should manifest itself the least for high radius ratios. Also, no hydrodynamic forces were included which, again, suggests that the results are more representative for drops of equal than different sizes. In other words, the results for very small drops of similar size should earn our trust.

In order to examine turbulent collision kernels for collector drops in the range 50-800 $\mu \mathrm{m}$ (larger than those which are of our principal interest), the diffusion equation for a stochastic process was applied by Reuter et al. [1988]. The rate of energy dissipation was varied from a few to a few thousand $\mathrm{cm}^{2} \mathrm{sec}^{-3}$. The basic equation of Reuter et al. was the diffusion equation in the frame moving with the collected drop. In cylindrical coordinates, $(r, \phi, z)$ this equation is

$$
\frac{\partial V}{\partial t}+\left(v_{2}-v_{1}\right) \frac{\partial V}{\partial z}+\sigma \frac{\partial^{2} V}{\partial z^{2}}+\sigma \frac{\partial^{2} V}{\partial r^{2}}=0
$$

where $V$ is the probability of collision between two drops in the period of time between $t$ and $T, v_{1}$ and $v_{2}$ the drop terminal velocities, and $\sigma$ the coefficient of turbulent diffusion. $V$ is a function of $t$ and the collector drop position in the moving frame which also represents the distance between drops. Note that because of symmetry 
of the isotropic and homogeneous turbulent field (which was assumed), there is no $\phi$-component of diffusion in the latter equation. (1.18) was solved with constant $\sigma$. Then, the solution was integrated over the cylindrical volume such that no collisions were possible between drops coming from outside of the volume during the specified time period. A slight increase in collision kernels was observed, especially for collector drops $50 \mu \mathrm{m}$ in radius.

In a follow-up article, Cooper \& Baumgardner [1989] criticized some of the Reuter at al. assumptions. In the first place, the assumption of constant diffusivity was disputed on the ground that the diffusion of drops depends on their relative distance. More clearly, the eddies which manipulate the diffusion are smaller than or equal to the distance between drops. Therefore, the size range of the participating eddies changes with the drop separation. Further, the integration of the probability $V$ was questioned. While integrating, Reuter et al. assumed that the concentration of the collector drops is constant. According to Cooper \& Baumgardner this is not the case because in the volume below the collected drop the concentration of the collector drops is lower due to the fact that some of the random trajectories already passed through the collected drop. Finally, Cooper \& Baumgardner pointed out that in the Reuter et al. approach, the motion relative to the air (the inertial effect) was not, included. The general conclusion of Cooper \& Baumgardner was that. Reuter et al. overestimated the effect of turbulence.

We agree with the criticism of Cooper \& Baumgardner. The additional comment about the method of Reuter et al. which we have concerns the representation of hydrodynamic forces. Reuter et al. assumed that the probability $V$ is the product of the probability without hydrodynamic interaction and the laminar collision efficiency. This may be incorrect. The laminar collision efficiency depends not only on the drop sizes but also on their relative velocity. With the ambient turbulent field, 
drops approach each other with velocities determined by both their sizes and masses, and the turbulent field. Also, the effects of shear and rotation due to turbulence were neglected.

\subsection{Modelling Drop Trajectories}

Modelling of drop trajectories is geared toward a proper description of hydrodynamic interactions. Levin \& Sedunov [1966] extended a method which has been traditionally used for a laminar flow (i.e. calculation of the grazing trajectory) to a turbulent case. The electrostatic forces were also included. Levin \& Sedunov assumed that turbulent mixing is effective only at large distances. Thus, turbulence can be neglected when the drops are in close proximity. Once the characteristic distance was defined, the calculation of 'turbulent' grazing trajectory started at the vertical distance between drops equal to the length parameter characterizing turbulence. The smallest scales of turbulence were neglected. The results pointed out the importance of turbulence. However, the turbulence representation seems to be oversimplified, and, in particular, there was no justification given for neglecting the smallest scales of turbulence.

De Almeida [1975, 1976, and 1979], went a very significant 'one step' further. He developed a method to model an instantaneous turbulent velocity field, and, then, calculated the trajectories of two drops. The hydrodynamic interactions between drops were included. The collision rates were calculated on a probabilistic basis by repeating the trajectory calculations numerous times for different realizations of the turbulent field. De Almeida's results were encouraging-the collision rates, for a collector drop $15 \mu \mathrm{m}$ in radius, increased by factor 10 or even 100 for energy dissipation rates as low as 1 or $10 \mathrm{~cm}^{2} \mathrm{sec}^{-3}$. However, there has been a significant amount of 
criticism of the method. Because de Almeida's research was novel in comparison to the other approaches, we will present both the criticism in the literature and our perspective of de Almeida's approach.

The turbulent field was modelled with the help of a Monte Carlo method. The constraints imposed upon the velocity field was that of the second order structure function for the longitudinal and transverse velocity components in the form

$$
\begin{gathered}
B_{r r}(r)=\overline{\left[v_{r}(x+r)-v_{r}(x)\right]}=C \epsilon^{\frac{2}{3} r^{\frac{2}{3}},} \\
B_{n n}(r)=\overline{\left[v_{n}(x+r)-v_{n}(x)\right]}=\frac{4}{3} C \epsilon^{\frac{2}{3} r^{\frac{n}{3}},}
\end{gathered}
$$

where $v_{r}$ and $v_{n}$ are respectively the longitudinal and transverse components of the velocity, and $C$ is an universal constant. Note that the ' $2 / 3$ ' law holds exclusively in the inertial subrange. Note also that de Almeida generated a two-dimensional turbulent field. The chosen value of $r$ was $0.001 \mathrm{~cm}$ which is well within the dissipation subrange for both values of the examined rate of energy dissipation (i.e. 1 and $10 \mathrm{~cm}^{2}$ $\left.\sec ^{-3}\right)$. Therefore, de Almeida's approach was criticized by Pruppacher \& Klett [1980] for using an inappropriate velocity correlation. (Clearly, the velocity field generated in such a way reveals high and chaotic variability within the range where motion should be correlated and velocity should be changing approximately lincarly with distance.) Also, it seems to us that constraining the motion to only two dimensions was not justified. Although, on average, the problem possesses certain symmetries, the individual trajectories are by no means confined to a plane. Finally, in the treatment of turbulence no effort was made to account for velocity-time correlations.

The trajectory calculations were performed by de Almeida with the help of the equation derived by Tchen [1949]. Tchen's equation was the first to describe the 
gravitational settling of a spherical particle in a nonuniform flow. It reads

$$
\begin{gathered}
\vartheta \rho_{p} \frac{d \mathbf{v}}{d t_{p}}=\underbrace{\vartheta\left(\rho_{p}-\rho_{f}\right) \mathrm{g}}_{\text {Gravity and buoyancy }}+\underbrace{\vartheta \rho_{f} \frac{d \mathbf{u}}{d t_{f}}}_{\text {Prcssurc gradient }}+\underbrace{\frac{1}{2} \vartheta \rho_{f}\left(\frac{d \mathbf{u}}{d t_{p}}-\frac{d \mathbf{v}}{d t_{p}}\right)}_{\text {Added mass }}+ \\
\underbrace{6 \pi \mu a(\mathbf{u}-\mathbf{v})}_{\text {Stokes' drag }}+\underbrace{\frac{a}{\sqrt{\pi \nu}} \int_{t_{0 p}}^{t_{p}}\left(\frac{d \mathbf{u}}{d \tau}-\frac{d \mathbf{v}}{d \tau}\right) \frac{d \tau}{\sqrt{t-\tau}}}_{\text {History term }},
\end{gathered}
$$

where $\vartheta$ is the mass of a particle, $a$ its radius, $\mathbf{u}$ and $\mathbf{v}$ are the velocities of the fluid and the particle respectively, and $t_{f}$ and $t_{p}$ denote time along the trajectory of the fluid parcel and the particle respectively. One of the assumptions while deriving this equation was that the density of particles is not much higher than that of the fluid. Because of the latter assumption, the use of Tchen's equation met with criticism from Pruppacher \& Klett [1980]. Here, however, we would like to defend de Almeida. Corrsin \& Lumley [1956], and Maxey \& Riley [1983] derived a similar equation (the latter from first principles.) The major disagreement between Tchen's version and the later versions of the equation was centred around the, so called, pressure gradient term. This term, however, as was shown by de Almeida, is small and can be neglected. (Altogether the pressure gradient, added mass, and history terms were neglected.) The next step of de Almeida seems to be more controversial. He formally substituted the Stokes' drag term in Tchen's equation by the expressions for Oseen drag derived by Klett \& Davis [1973], which represents the hydrodynamic interactions. No formal derivation was given. (Klett \& Davis solved the laminar problem with the inertial effects included in the form of Oseen's correction to the Stokes' drag.) Finally, Pruppacher \& Klett noticed that the results of de Almeida for the laminar case do not agree with those previously obtained by Klett \& Davis. The latter criticism undermines as well the trust for de Almeida's calculations.

The results obtained by de Almeida showed a dramatic increase of the collision efficiencies for the turbulent case, in particular, for small collector drops (of radii 15 
and $20 \mu \mathrm{m}$ ), and for the radius ratio $>0.3$. It is difficult to assess the nature of the errors due to calculation of hydrodynamic interactions. What we can only say is that the method of Klett \& Davis [1973] is inherently not appropriate for very small drops (say $15 \mu \mathrm{m}$ in radius). Further, the effect of turbulence may liave been magnified by the application of the velocity correlations characterizing the inertial subrange in the dissipation subrange. A qualitative lesson to be learnt, however, is that smallamplitude spatial variations of velocity (the rate of energy dissipation equal to $1 \mathrm{~cm}^{2}$ $\mathrm{sec}^{-3}$ ) may be able to influence the relative drop trajectories in such a way that the cumulative effect leads to an increase in collision efficiencies.

\subsection{Experimental Evidence}

One would hope that a reasonably designed experiment could resolve the dilemma whether or not turbulence enhances collisions between drops. In a series of experiments, Woods, Drake \& Goldsmith [1972], and Jonas \& Goldsmith [1972], examined collisions of small drops under the influence of a uniform shear flow. They argued that the uniform shear represents turbulence relatively well when the drops are in close proximity. More precisely, the power spectra of the velocity derivatives have maxima near the wavelength corresponding to the Kolmogorov length scale. If, additionally, those spectra are sufficiently narrow, the uniform shear representation may perform fairly well. The major finding of Jonas \& Goldsmith was that collision efficiencies increase appreciably for small collector drops $(10-15 \mu \mathrm{m})$ for values of shear from 8 to $18 \mathrm{sec}^{-1}$. However, they were not able to reproduce the experimental results by theoretical calculations for laminar shear flow. Later, Tennekes \& Woods [1972] demonstrated that even if the uniform shear represents a 'dissipative filarnent', the velocity distributions inside filaments play a major role in enhancing collision 
rates. Therefore, the experimental results for a shear flow should be interpreted cautiously. There are no other attempts at experimental work in this area known to the author. 


\section{2 \\ Scale Analysis}

Scale analysis is an invaluable first-step approach to the majority of fluid dynamics problems. In this chapter we try to assess the importance of the factors involved in the process of collisions as well as to justify the methods with which to investigate collision rates.

\subsection{Cloud Droplets}

Let us start the analysis with a single drop in still air under the influence of gravity. Clearly, the radius of the drop represents the length scale and the drop terminal velocity, the velocity scale. Small cloud droplets are of the principal concern in this work, therefore, we assume the range of radii from 2 to $20 \mu \mathrm{m}$. An isolated droplet under the above circumstances moves with the, so called, terminal velocity. This results from the equilibrium between the gravity-buoyancy force and the hydrodynamic drag force. The drag for drops as small as those considered is well represented by the Stokes formula

$$
D=6 \pi \mu r v_{t}
$$

where $\mu$ is the dynamic viscosity, $r$ the drop radius, and $v_{\ell}$ the drop terminal velocity. Equating the above with the formula for gravity and buoyancy

$$
G=\frac{4}{3} \pi r^{3}\left(\rho-\rho_{a i r}\right) g
$$


where $\rho$ and $\rho_{\text {air }}$ are the densities of water and air respectively, and $g$ is gravity, leads to the following expression for the drop terminal velocity

$$
v_{\iota}=\frac{2 g}{9 \mu}\left(\rho-\rho_{\text {air }}\right) r^{2}
$$

For example, the terminal velocity for a $15 \mu \mathrm{m}$ drop at standard temperature and pressure is equal to $2.7 \mathrm{~cm} \mathrm{sec}^{-1}$. Table 2.1 lists values for other droplet radii. The Reynolds numbers defined as

$$
\operatorname{Re}=\frac{r \cdot v_{t}}{\mu \rho}
$$

are listed in Table 2.1 as well. Let us just note that for a $15 \mu \mathrm{m}$ drop the Reynolds number is $0.01-$ a small Reynolds number indeed. Certainly the fact that the Reynolds number is small restricts our methods of treating the hydrodynamics to the 'convenient' low-Reynolds-number flows.

The time scale of a drop settling under the influence of gravity is represented by the drop relaxation time, i.e. time needed for the initially non-moving drop to reach roughly $60 \%$ of its terminal velocity $\left(1-\frac{1}{e}\right.$ factor.) The solution of the equation

$$
m \frac{d v}{d t}+6 \pi \mu v r-m g=0
$$

where $m$ is the mass of the drop, is

$$
v=\tau g\left[1-\exp \left(-\frac{\tau}{t}\right)\right] \quad \text { where } \quad \tau=\frac{m}{6 \pi \mu}
$$

$\tau$ is called the relaxation time. For a $15 \mu \mathrm{m}$ drop the relaxation time is $0.003 \mathrm{sec}$ (again, more values can be found in Table 2.1.) The inverse of the relaxation time is often called the response frequency, $\alpha=1 / \tau$. Further, one can introduce the nondimensional Stokes number

$$
\text { Stk }=\frac{\tau U}{L}
$$

where $U$ and $L$ represent respectively the characteristic length and velocity scales of the external flow. Small Stokes numbers characterize situations where the particle 
inertia has a small influence on the particle motion. When the Stokes number is small, the inertia of the drop ir; small and the drop response to the changes in the surrounding fluid is rapid. The opposite is true when the Stokes number is large. For the scales of turbulence which are of interest here, the Stokes numbers are small (for a $15 \mu \mathrm{m}$ drop and the rate of energy dissipation $\left.10 \mathrm{~cm}^{2} \mathrm{sec}^{-3}, \mathrm{Stk}=0.02\right)$ moaning that if not for gravity, drops would follow the flow field closely. More values of the Stokes number can be found in Table 2.1. The time and length scales of turbulence will be defined in the following $s$ cion.

A two-drop ensemble calls for more scales to be defined. In the first place, there is the distance between the centres $(D)$ as well as between the surfaces $(S)$. At. large separations, the Reynolds number based on $D$ is not much smaller than 1 , and higher order, Oseen representation of the drag force should be considered (as in Klett. \& Davis, 1973.) Note that the initial distance between centres required for laminar calculations is of the order of hundred radii of the collector drop. Fortunately, as shown in Chapter 8 , the interactions between droplets at large distances is negligible as compared to their relative motion caused by turbulence. Therefore, it is not, essential that the Oseen corrections be included in this research.

The distance between surfaces requires special attention at close separations. This is when molecular interactions come into the play. The common practice while numerically examining collisions between drops is to define a collision event as occurring when

$$
S=\varepsilon r_{1}
$$

where $r_{1}$ is the radius of the collector drop, and $\varepsilon$ is a small number, usually of the order of $10^{-4}-10^{-3}$. If the small gap between the surfaces of the drops is comparable with the mean molecular free path, $\lambda$, the assumption of continuity breaks down, and hydrodynamic models are no longer valid. The ratio of the free molecular path to 
the characteristic length scale of the flow is referred to as the Knudsen number. For the atmosphere $\lambda=0.06 \mu \mathrm{m}$. Let us calculate the Knudsen number (K) for a $15 \mu \mathrm{m}$ drop and $\varepsilon=10^{-3}$.

$$
K=\frac{\lambda}{S}=\frac{\lambda}{\varepsilon r_{1}}=4
$$

Certainly, we are in the regime where the assumption of continuity may not be valid (see also Table 2.1.) However, experiments showed [Jonas \& Goldsmith, 1972] that for collector drops as small as $20 \mu \mathrm{m}, \varepsilon=10^{-3}$ is the appropriate value (i.e. the assumption of continuity still holds.) Higher values of $\varepsilon$ are perhaps better for $10 \mu \mathrm{m}$ collector drops. Still, in order to facilitate comparisons with other researches, we will apply the lower number.

Finally, let us introduce the length and time scales of interaction between two different sized drops. Due to their different terminal velocities, drops proceeding with their gravity-determined motion are 'in contact' over a finite time-we call it time of interaction. During that time the larger drop relocates itself by the distance which can be calculated from its terminal velocity-this is the distance of interaction. Before proceeding with the arithmetic expressions of the above, we define the distance between drops where they start to feel their mutual presence. According to convention [Jonas \& Goldsmith, 1972], this distance for such small drops as those considered here is of the order of 10 radii of the collector drop. Physically, this means that at that distance, the velocity generated by an isolated drop moving with constant velocity in the still fluid is equal to $1 / 2 e$ of the drop velocity (based upon the Stokes solution for an isolated drop.) Therefore, the time of interaction between drops is

$$
\mathcal{T}=\frac{10 r_{1}}{v_{1}-v_{2}}
$$

where $v_{1}$ and $v_{2}$ are the terminal velocities of the collector and collected drop respectively. (Note that the difference in terminal velocities represents the velocity scale.) 
Further, the distance of interaction is

$$
\mathcal{L}=\frac{\left(10 r_{1}\right) v_{1}}{v_{1}-v_{2}}
$$

In Table 2.2 there are tabulated values of the time and length scales of interaction for different sizes of drops. In the next section we will compare those values with the appropriate scales of turbulence.

TABLE 2.1

Characteristic Scales of a Single Drop

\begin{tabular}{cccccc}
\hline $\begin{array}{c}\text { Radius } \\
(\mu \mathrm{m})\end{array}$ & $\begin{array}{c}\text { Terminal velocity } \\
\left.v_{t}(\mathrm{~cm} \mathrm{sec})^{-1}\right)\end{array}$ & $\begin{array}{c}\text { Reynolds number } \\
\operatorname{Re}\end{array}$ & $\begin{array}{c}\text { Relaxation time } \\
\tau(\mathrm{sec})\end{array}$ & $\begin{array}{c}\text { Stk for } \\
\epsilon=1 \mathrm{~cm}^{2} \mathrm{sec}^{-3}\end{array}$ & $\begin{array}{c}\text { Stk for } \\
\epsilon=100 \mathrm{~cm}^{2} \mathrm{sec}^{-3}\end{array}$ \\
\hline 30 & 10.7 & 0.2 & 0.01 & 0.03 & 0.3 \\
20 & 4.8 & 0.06 & 0.005 & 0.01 & 0.1 \\
15 & 2.7 & 0.03 & 0.003 & 0.007 & 0.07 \\
10 & 1.2 & 0.008 & 0.001 & 0.003 & 0.03 \\
5 & 0.3 & 0.001 & 0.0003 & 0.0008 & 0.008 \\
2 & 0.05 & 0.00006 & 0.00005 & 0.0001 & 0.001 \\
\hline
\end{tabular}

TABLE 2.2

\section{Characteristic Scales of Two-Drop Interactions}

\begin{tabular}{|c|c|c|c|c|c|}
\hline $\begin{array}{l}\text { Radius } r_{1} \\
\qquad(\mu \mathrm{m})\end{array}$ & $\begin{array}{l}\text { Radius } r_{2} \\
\quad(\mu \mathrm{m})\end{array}$ & $\begin{array}{c}\text { Velocity scaje } \\
v_{1}-v_{2}(\mathrm{~cm} \mathrm{sec}-1)\end{array}$ & $\begin{array}{l}\text { Lengtla scale } \\
\qquad \mathcal{L}(\mathrm{cm})\end{array}$ & $\begin{array}{l}\text { Tine scale } \\
\mathcal{T} \text { (sec) }\end{array}$ & $\begin{array}{c}\text { Kutudsen number } \\
\mathrm{K}\end{array}$ \\
\hline 30 & 29.6 & 0.3 & 1.13 & 0.11 & 2 \\
\hline 30 & 15 & 8.0 & 0.040 & 0.0037 & \\
\hline 30 & 4 & 10.5 & 0.031 & 0.0029 & \\
\hline 20 & 19.7 & 0.1 & 0.67 & 0.14 & 3 \\
\hline 20 & 10 & 3.6 & 0.027 & 0.0056 & \\
\hline 20 & 3 & 4.7 & 0.021 & 0.0043 & \\
\hline 15 & 14.8 & 0.07 & 0.57 & 0.21 & 4 \\
\hline 15 & 8 & 1.9 & 0.021 & 0.0078 & \\
\hline 15 & 2 & 2.6 & 0.015 & 0.0057 & \\
\hline 10 & 9.9 & 0.02 & 0.50 & 0.42 & 6 \\
\hline 10 & 5 & 0.9 & 0.013 & 0.011 & \\
\hline 10 & 2 & 1.1 & 0.010 & 0.0088 & \\
\hline
\end{tabular}




\subsection{Cloud Turbulence}

Even to the naked eye clouds appear turbulent. Yet, rigorous measurements of the degree of cloud turbulence have only become available in the last thirty years or so with the help of airborne instruments. With high frequency measurements of velocity, the velocity spectrum, and, consequently, the rate of energy dissipation in clouds have been obtined in a number of flights. The measurements which we are going to refer to are Ackerman [1967 and 1968], MacPherson [1977], and MacPherson $\&$ Isaac [1979]. The values of the rate of energy dissipation obtained vary between a few to a few hundred $\mathrm{cm}^{2} \mathrm{sec}^{-3}$. As expected, the higher values are representative of the turbulence in cumulus storm clouds while the lower values are representative of stratus clouds [Ackerman,1967.] Furthermore, the measurements of MacPherson \& Isaac [1979] show that in summer continental cumulus clouds, the values of $\epsilon$ are generally lower near the cloud base $\left(20-80 \mathrm{~cm}^{2} \mathrm{sec}^{-3}\right)$ than near the cloud top (up to $400 \mathrm{~cm}^{2} \mathrm{sec}^{-3}$.) In this research, the primary goal is to investigate collision rates in developing cumulus clouds in which the buoyancy driven turbulence has not yet reached its maximum strength. The rate of energy dissipation near cloud base seems to better represent young clouds. Therefore, the values from 1 to $100 \mathrm{~cm}^{2} \mathrm{sec}^{-3}$ are investigated. Also, the above values have been considered by other researches thus we will be able to relate our results to previous work. However, in order to find the lower limit of the rate of energy dissipation which still influences the collision rates, the lower values, 0.1 and $0.01 \mathrm{~cm}^{2} \mathrm{sec}^{-3}$ were also included.

It is appropriate for the problem at hand to restrict our interest to only the smallest scales of turbulence. We are exclusively interested how turbulence modifies drop trajectories, and, consequently, collision rates on the scale where drops interact hydrodynamically. (Some researches examined the effect of turbulence on cloud drop 
spectra through large scale mixing processes of cloudy and cloud-free air. These either involved turbulence parameterization alone [Baker \& Latham, 1982] or both turbulence parameterization and gravitational sedimentation [Baker et al., 1984; Jensen \& Baker, 1989]. The influence of large scale, turbulent mixing on collision rates is not considered here.) The characteristic scales, then, are defined respectively by the Kolmogorov length, time, and velocity as follows

$$
\eta_{K}=\left(\frac{\nu^{3}}{\epsilon}\right)^{\frac{1}{4}} \quad \tau_{K}=\left(\frac{\nu}{\epsilon}\right)^{\frac{1}{2}} \quad v_{K}=(\nu \epsilon)^{\frac{1}{d}}
$$

where $\epsilon$ is the rate of energy dissipation. For $\epsilon=100 \mathrm{~cm}^{2} \mathrm{sec}^{-3}$, the above quantities are $\eta_{K}=0.14 \mathrm{~cm}, \tau_{K}=0.12 \mathrm{sec}$, and $v_{K}=1.11 \mathrm{~cm} \mathrm{sec}^{-1}$. More values can be found in Table 2.3.

The comparison of turbulence scales with those characterizing a pair of drops clearly points their similarity. In terms of spectra, the action takes place in the upper range of the inertial subrange and in the dissipation subrange-and this is where our model of turbulence must perform flawlessly.

TABLE 2.3

Characteristic Scales of Turbulence

\begin{tabular}{cccc}
\hline $\begin{array}{c}\text { Energy dissipation rate } \\
\epsilon\left(\mathrm{cm}^{2} \mathrm{sec}^{-3}\right)\end{array}$ & $\begin{array}{c}\text { Kolmogorov length } \\
\eta_{K}(\mathrm{~cm})\end{array}$ & $\begin{array}{c}\text { Kolmogorov time } \\
\tau_{K}(\mathrm{scc})\end{array}$ & $\begin{array}{c}\text { Kolmogorov velocity } \\
v_{K}\left(\mathrm{~cm} \mathrm{sec}^{-1}\right)\end{array}$ \\
\hline 0.01 & 0.76 & 3.87 & 0.20 \\
0.1 & 0.43 & 1.22 & 0.35 \\
1 & 0.24 & 0.39 & 0.62 \\
10 & 0.14 & 0.12 & 1.11 \\
100 & 0.08 & 0.04 & 1.97 \\
1000 & 0.04 & 0.01 & 3.50 \\
\hline
\end{tabular}




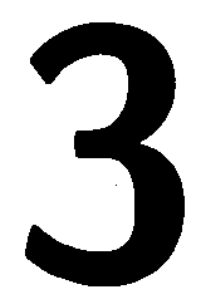

\section{Microhydrodynamics of Cloud Droplets}

In this chapter, we concern ourselves with the motion of the drops. We present a model with which to calculate trajectories of pairs of drops. Our model is based on the assumptions that drops behave like rigid spheres and are small enough to calculate forces acting upon them with help of the low-Reynolds-number approach.

\subsection{The Low-Reynolds-Number Approach}

If the inertia of a drop is small (indeed this is the case as we will show later) gravity and the hydrodynamic resistance are the only forces acting on the drops. These forces, according to Newton's second law, determine the motion. Our task is to find the resistance. The problem has an additional dimension of complexity because of the hydrodynamic interactions between two drops. The hydrodynamic forces can be easily obtained if the solution to the boundary value problem of the form

$$
\frac{\partial \mathbf{u}}{\partial t}+\mathbf{u} \cdot \nabla \mathbf{u}=-\frac{1}{\rho} \nabla p+\nu \nabla^{2} \mathbf{u}, \quad \nabla \cdot \mathbf{u}=0
$$

is known. Because solution of the full Navier-Stokes equation is by no means trivial, we apply scale analysis to prove that our problem can be tackled by solving a linear problem governed by the Stokes equation. Let $U, l$, and $\tau$ be the characteristic 
velocity, length, and time scale respectively. Then, (3.1) in a nondimensional form is

$$
\operatorname{ReSl} \frac{\partial \mathbf{u}^{\prime}}{\partial t^{\prime}}+\operatorname{Re} \mathbf{u}^{\prime} \cdot \nabla^{\prime} \mathbf{u}^{\prime}=-\nabla^{\prime} p^{\prime}+\nabla^{\prime 2} \mathbf{u}^{\prime}, \quad \nabla^{\prime} \cdot \mathbf{u}^{\prime}=0
$$

where prime denotes nondimensional quantities, and the Reynolds and the Strouhal numbers characterizing the problem are

$$
\operatorname{Re}=\frac{U l}{\nu} \quad \text { and } \quad \mathrm{Sl}=\frac{l}{U \tau}
$$

For a drop which settles under the influence of gravity, the characteristic velocity is its terminal velocity while the characteristic length, its radius. Settling in a still fluid is a time independent process, but the external turbulent field introduces time variation which may be characterized by the viscous cutoff of the time spectrum (nota bene equal to the Kolmogorov time scale.) For the rate of encrgy dissipation $\epsilon=100 \mathrm{~cm}^{2} \mathrm{sec}^{-3}$, the associated time scale is $0.04 \mathrm{sec}$. A typical $15 \mu \mathrm{m}$ drop has a terminal velocity equal to $2.7 \mathrm{~cm} \mathrm{sec}^{-1}$. Introducing the above numbers in (3.3), we get $\mathrm{Re}=0.03$ and $\mathrm{Sl}=0.02$. Clearly, the terms on the left hand side of (3.2) are small in comparison to the terms on the right hand side. Therefore, (3.1) takes the form of the time-independent Stokes equation

$$
\mu \nabla^{2} \mathbf{u}=\nabla p \quad \nabla \cdot \mathbf{u}=0
$$

In the presence of potential forces like gravitational or electrostatic forces, Eclu. (3.4) retains its form as these forces can be formally incorporated in the pressure term.

The underlying assumption necessary to proceed with the solution of the Stokes equation which has not been addressed so far is that concerning the rigidity of drops. It is known that internal circulation develops inside drops falling through another immiscible liquid (see for example Pruppacher \& Beard [1970].) Due to internal circulation, the drag on a drop increases. Comparisons of experiments performed with rigid spheres [Perry, 1950] and water drops [Beard \& Pruppacher, 1969] show 
satisfactory agreement between drag on a sphere and on a drop of the same size up to radii of $500 \mu \mathrm{m}$. Therefore, we feel justified to use the rigid-sphere approximation, and in the following section we will refer to spheres rather than to drops.

\subsection{The Resistance Problem}

The linear character of the Stokes equation has beneficial consequences for the process of obtaining hydrodynamic forces, couples, and stresslets. The last are of no interest to us, yet are considered just for the sake of completeness. Let us exparid the ambient flow in a Taylor series. The two first terms of the series are

$$
u_{i}(\mathbf{x})=u_{i}\left(\mathbf{x}_{0}\right)+\frac{\partial u_{i}}{\partial x_{j}} \delta x_{j}+o\left(\delta^{2} x\right)
$$

Upon defining the rate of stress tensor and the solid body angular velocity as

$$
\mathrm{E}_{\infty}=E_{i j}^{\infty}=\frac{1}{2}\left(\frac{\partial u_{i}}{\partial x_{j}}+\frac{\partial u_{j}}{\partial x_{i}}\right) \quad \text { and } \quad \Omega_{\infty}=\Omega_{k}^{\infty}=\frac{1}{2}\left(\frac{\partial u_{i}}{\partial x_{j}}-\frac{\partial u_{j}}{\partial x_{i}}\right)
$$

respectively, (3.5) results in

$$
u_{i}=u_{i}\left(\mathbf{x}_{0}\right)+\mathbf{E}_{\infty} \cdot \delta \mathbf{x}+\Omega_{\infty} \times \delta \mathbf{x}
$$

After Kim \& Karrila [1991], the forces, couples, and stresslets for a flow in the form of $(3.7)$, can be represented by the following matrix equation

$$
\left(\begin{array}{c}
\mathbf{F}_{1} \\
\mathbf{F}_{2} \\
\mathbf{T}_{1} \\
\mathbf{T}_{2} \\
\mathbf{S}_{1} \\
\mathbf{S}_{2}
\end{array}\right)=-\mu\left(\begin{array}{llllll}
\mathbf{A}_{11} & \mathbf{A}_{12} & \widetilde{\mathbf{B}}_{11} & \widetilde{\mathbf{B}}_{12} & \widetilde{\mathbf{G}}_{11} & \widetilde{\mathbf{G}}_{12} \\
\mathbf{A}_{21} & \mathbf{A}_{22} & \widetilde{\mathbf{B}}_{21} & \widetilde{\mathbf{B}}_{22} & \widetilde{\mathbf{G}}_{21} & \widetilde{\mathbf{G}}_{22} \\
\mathbf{B}_{11} & \mathbf{B}_{12} & \mathbf{C}_{11} & \mathbf{C}_{12} & \widetilde{\mathbf{H}}_{11} & \widetilde{\mathbf{H}}_{12} \\
\mathbf{B}_{21} & \mathbf{B}_{22} & \mathbf{C}_{21} & \mathbf{C}_{22} & \widetilde{\mathbf{H}}_{21} & \widetilde{\mathbf{H}}_{22} \\
\mathbf{G}_{11} & \mathbf{G}_{12} & \mathbf{H}_{11} & \mathbf{H}_{12} & \mathbf{M}_{11} & \mathbf{M}_{12} \\
\mathbf{G}_{21} & \mathbf{G}_{22} & \mathbf{H}_{21} & \mathbf{H}_{22} & \mathbf{M}_{21} & \mathbf{M}_{22}
\end{array}\right)\left(\begin{array}{c}
\mathbf{v}_{1}-\mathbf{u}\left(\mathbf{x}_{1}\right) \\
\mathbf{v}_{2}-\mathbf{u}\left(\mathbf{x}_{2}\right) \\
\mathbf{\Omega}_{1}-\mathbf{\Omega}_{\infty} \\
\mathbf{\Omega}_{2}-\mathbf{\Omega}_{\infty} \\
-\mathbf{E}_{\infty} \\
-\mathbf{E}_{\infty}
\end{array}\right),
$$

where $\mathbf{A}, \mathbf{B}$, and $\mathbf{C}$ are second-rank tensors, $\mathbf{G}$ and $\mathbf{H}$ are third-rank tensors, and $\mathbf{M}$ is a fourth-rank tensor. The matrix in (3.8) is called the grand matrix. One can prove that the following symmetry relations hold [Kim \& Karrila, 1991]

$$
A_{i j}^{\alpha \beta}=A_{j i}^{\beta \alpha}
$$




$$
\begin{aligned}
B_{i j}^{\alpha \beta} & =\widetilde{B}_{j i}^{\beta \alpha} \\
C_{i j}^{\alpha \beta} & =C_{j i}^{\beta \alpha} \\
G_{i j k}^{\alpha \beta} & =\widetilde{G}_{k i j}^{\beta \alpha} \\
H_{i j k}^{\alpha \beta} & =H_{k i j}^{\beta \alpha} \\
M_{i j k l}^{\alpha \beta} & =M_{k l i j}^{\beta \alpha} .
\end{aligned}
$$

Note that in the above equations superscripts $\alpha$ and $\beta$ denote droplets and corresponds to subscripts 1 and 2 in (3.8). Also, the grand matrix is positively defined.

The geometry of two drops possesses an intrinsic symmetry-the line through the centres being an axis of symmetry. Because of this the tensors contained in the grand matrix can be easily expressed with the help of scalar resistance functions as follows [Kim \& Karrila, 1991]

$$
\begin{gathered}
A_{i j}^{\alpha \beta}=X_{\alpha \beta}^{A} d_{i} d_{j}+Y_{\alpha \beta}^{A}\left(\delta_{i j}-d_{i} d_{j}\right) \\
B_{i j}^{\alpha \beta}=Y_{\alpha \beta}^{B} \epsilon_{i j k} d_{k} \\
C_{i j}^{\alpha \beta}=X_{\alpha \beta}^{C} d_{i} d_{j}+Y_{\alpha \beta}^{C}\left(\delta_{i j}-d_{i} d_{j}\right) \\
G_{i j k}^{\alpha \beta}=X_{\alpha \beta}^{G}\left(d_{i} d_{j}-\frac{1}{3} \delta_{i j}\right)+Y_{\alpha \beta}^{G}\left(d_{i} \delta_{j k}+d_{j} \delta_{i k}-2 d_{i} d_{j} d_{k}\right) \\
H_{i j k}^{\alpha \beta}=Y_{\alpha \beta}^{H}\left(\epsilon_{i k l} d_{j}+\epsilon_{j k l} d_{i}\right) d_{l},
\end{gathered}
$$

where vector $d_{i}$ is the unit vector along the line of centres. (An analogous expression for $M_{i j k l}$ can be found in Kim \& Karrila [1991].) Now the scalar resistance functions $X_{\alpha \beta}$ and $Y_{\alpha \beta}$ have a very straightforward association with simple types of motion such as motion along the line of centers, perpendicular to the line of centers, rotation, etc.

Before proceeding with the explanation of the methods which are available to calculate the grand matrix coefficients, we formally write the set of equations which 
completely describe the motion of two interacting droplets

$$
\begin{gathered}
m_{\alpha} \frac{d v_{i}^{\alpha}}{d t}=F_{i}^{\alpha}+m_{\alpha} g_{i}, \\
I_{\alpha} \frac{d \Omega_{i}^{\alpha}}{d t}=T_{i}^{\alpha} \\
\frac{d x_{i}^{\alpha}}{d t}=v_{i}^{\alpha}, \\
\text { where } \\
F_{i}^{\alpha}=\mu A_{i j}^{\alpha \alpha}\left[u_{j}\left(\mathbf{x}^{\alpha}\right)-v_{j}^{\alpha}\right]+\mu A_{i j}^{\alpha \beta}\left[u_{j}\left(\mathbf{x}^{\beta}\right)-v_{j}^{\beta}\right]-\mu \widetilde{B}_{i j}^{\alpha \alpha}\left(\Omega_{j}^{\alpha}-\Omega_{j}^{\infty}\right)-\mu \tilde{B}_{i j}^{\alpha \beta}\left(\Omega_{j}^{\beta}-\Omega_{j}^{\infty}\right) \\
+\mu \widetilde{G}_{i j k}^{\alpha \alpha} E_{j k}^{\infty}+\mu \widetilde{G}_{i j k}^{\alpha \beta} E_{j k}^{\infty}, \\
T_{i}^{\alpha}=\mu B_{i j}^{\alpha \alpha}\left[u_{j}\left(\mathbf{x}^{\alpha}\right)-v_{j}^{\alpha}\right]+\mu B_{i j}^{\alpha \beta}\left[u_{j}\left(\mathbf{x}^{\beta}\right)-v_{j}^{\beta}\right]-\mu C_{i j}^{\alpha \alpha}\left(\Omega_{j}^{\alpha}-\Omega_{j}^{\infty}\right)-\mu C_{i j}^{\alpha \beta}\left(\Omega_{j}^{\beta}-\Omega_{j}^{\infty}\right) \\
+\mu \widetilde{H}_{i j k}^{\alpha \alpha} E_{j k}^{\infty}+\mu \tilde{H}_{i j k}^{\alpha \beta} E_{j k}^{\infty} .
\end{gathered}
$$

\subsection{Solutions of the Stokes Equation}

Since the beginning of this century, a wide variety of methods have been applied in order to obtain the flow around two spheres. These are cispherical coordinates methods [Stimson \& Jeffery, 1926; Wakiya, 1967; Davis,1969; O'Neill \& Mujumdar, 1971a, b], methods of reflections [Happel \& Brenner, 1966, Jeffrey \& Onishi, 1984; Jeffrey, 1992], asymptotic methods [O'Neill \& Stewartson, 1967; Jeffrey, 1982], and collocation methods [Ganatos, Pfeffer \& Weinbaum, 1978]. It is not our objective to present a thorough analysis of all these methods, however, the ones which are directly applied in this research will be described in some detail.

In order to find the resistance coefficients, the following steps are commonly undertaken. In the first place, the resistance problem has to be decomposed in such a way that coefficients in the grand matrix (3.8) can be associated with forces and 
torques for the particular type of motion. (Linearity of the Stokes equation assures that this can be done.) Then, the Stokes equation (3.4) is solved with the boundary conditions applied to the surfaces of both spheres. In the solutions presented below no-slip boundary conditions are assumed on the spheres meaning that the velocity on the surface of the body is equal to that of the body surface. The case of slip-flow boundary conditions will be discussed in the next section. With the resolved velocity field around the spheres, forces and torques upon the spheres due to the flow are calculated as surface integrals

$$
\begin{gathered}
\mathbf{F}_{\alpha}=\int_{S_{\alpha}} \sigma \cdot d \mathbf{S}, \\
\mathbf{T}_{\alpha}=\int_{S_{\alpha}}\left(\mathbf{x}-\mathbf{x}_{\alpha}\right) \times \sigma \cdot d \mathbf{S},
\end{gathered}
$$

where $\sigma$ is the stress tensor and $\mathbf{x}_{\alpha}$ is the centre of the sphere.

\subsubsection{Method of Bispherical Coordinates}

This method is based on introducing special orthogonal coordinates $(\eta, \xi, \zeta)$ in such a way that the absolute value of $\eta$ is constant on the surface of each of the spheres. Inherently, $\eta$ is positive on the surface of one of the spheres while negative on the surface of the other. The relations between cylindrical and bispherical coordinates can be found in Happel \& Brenner [1965]. The solution of the Stokes equation in bispherical coordinates is exact, although represented by an infinite series. Note that. the major advantage of special coordinates, in this case bispherical coordinates, is the formulation of the boundary conditions on the surfaces of the spheres. 


\subsubsection{Axisymmetric Problem}

Let us assume that droplets are placed on the $z$-axis. Also, let the drops move along the $z$-axis. Then, the coefficients $X_{\alpha \beta}^{A}$ represent flows axisymmetric about the line of centers. This axisymmetric problem was worked out with the help of bispherical coordinates by Stimson \& Jeffery [1926]. They considered only the case of equal velocities, $v_{1}=v_{2}$. Because the problem is axisymmetric they introduced Stokes' stream function for cylindrical coordinates. The solution, then, amounts to solving the vorticity equation. After introducing special, bispherical coordinates, the analytical solution was represented as an infinite series involving Legendre functions. Maude [1961], showed that the Stimson \& Jeffery [1926] solution can be easily extended to the case of $v_{1}=-v_{2}$, and, therefore, the general case of arbitrary velocities can be solved as well (because of linearity of Stokes equation.)

\subsubsection{Problems with a Plane of Symmetry}

In this case, while $z$-axis is the line of centres, both spheres move in the $x$ direction and rotate about $y$-axis. Clearly, the $z-x$ plane is the plane of symmetry. Notice that considering just translation allows us to calculate coefficients $Y_{\alpha \beta}^{A}$ and $Y_{\alpha \beta}^{C}$ while considering just rotation allows us to calculate coefficients $\tilde{Y}_{\alpha \beta}^{B}$.

The methodology of a solution to the problem possessing a plane symmetry was developed by Dean \& O'Neill [1963] and O'Neill [1964], and later applied to the problem under consideration by Wakiya [1967] and Davis [1969]. In cylindrical coordinates, the solutions for pressure and velocity components are in the form

$$
p=\frac{\mu}{c} \sum_{m=0}^{\infty} Q_{m} \cos m \phi, \quad v_{r}=\frac{1}{2} \sum_{m=0}^{\infty}\left(\frac{r}{c} Q_{m}+u_{m}+v_{m}\right) \cos m \phi
$$




$$
v_{\phi}=\frac{1}{2} \sum_{m=0}^{\infty}\left(u_{m}-v_{m}\right) \sin m \phi, \quad v_{z}=\frac{1}{2} \sum_{m=0}^{\infty}\left(\frac{z}{c} Q_{m}+2 w_{m}\right) \cos m \phi,
$$

where $c$ is a constant, and $Q_{m}, u_{m}, v_{m}$, and $w_{m}$ are functions of $r$ and $z$ only. After the transformation from cylindrical to bispherical coordinates, solutions for the above functions were found in the form of infinite series of Legendre poiynomials.

\subsubsection{Method of Reflections}

Some problems cannot be easily treated with help of special coordinatics. The method of reflections is one of the most commonly applied approximate methods but. only for large separations. The method was developed by Smoluchowski [1911]. The essence of the approach consists of the assumption that the ambient ficld around $\Omega$ particle is composed of the original field and the disturbance produced by the other particle. The iterative process leads to a solution in the form of an infinite series.

Jeffrey [1992] used the method of reflections to obtain the solutions for the case of the uniform straining motion for large drop separations. The solutions were obtained separately for the case of axisymmetric straining motion-along the line of centers, $z$-axis in our notation (coefficients $X_{\alpha \beta}^{G}$ ) and transverse to the line of centers (coefficients $Y_{\alpha \beta}^{G}$ and $X_{\alpha \beta}^{Y}$.) The general Lamb's solution of Stokes equation [1932] served as a starting point. This is a solution in spherical coordinates in the form of the infinite series of solid harmonics. For the problem at hand, Lambs' solution was written for both pressure and velocity outside each of the spheres (hence the method is called twin multipole expansion.) The pressure and velocity fields were then sought, as superpositions of fields around a single sphere

$$
\mathbf{v}=\mathbf{v}^{(1)}+\mathbf{v}^{(2)} \quad p=p^{(1)}+p^{(2)}
$$

Following Happel $\propto$ Brenner [1965], boundary conditions were applied to the following 
three scalar functions

$$
\frac{\mathbf{r}_{\alpha}}{r} \cdot \mathbf{u} \quad r_{\alpha} \nabla \cdot \mathbf{u} \quad \mathbf{r}_{\alpha} \cdot \nabla \times \mathbf{u}
$$

rather than directly to the velocity.

Because of slow convergence for close proximity of the spheres, twin multipole expansion is mainly applicable to cases of large drop separations. In the papers by Jeffrey \& Onishi [1984] and Jeffrey [1992] this method combined with an asymptotic method was used to estimate all the coefficients of the grand matrix for arbitrary separations.

\subsubsection{Asymptotic Methods}

Asymptotic methods were introduced to the problems involving hydrodynamic interactions by O'Neill \& Stewartson [1967] for the case of a sphere moving parallel to a plane. They performed matched asymptotic expansion with the 'inner' solution valid near the small gap between the plane and the sphere and the 'outer' solution valid in the remainder of the fluid. Later, however, it was found that despite the fact that a complete analysis can be obtained only by considering the entire flow, the analysis of the flow in the gap alone is sufficient to find the form of leading singular terms [Cooley \& O'Neill, 1969.]

Jeffrey [1989] analyzed the case of two spheres moving along the line of centers when the spheres are very close. The nondimensional gap between the spheres is small, $\varepsilon \ll 1$. The solution for the stream function was sought in the form of power series in $\varepsilon$. Later [Jeffrey: 1989 and 1991], the asymptotic analysis was applied to the probiem of two spheres in a two-dimensional pure straining motion. Because of symmetries of the grand matrix, the solution was obtained for the equivalent problem 
of the flow between two deforming spheres. (In the most r.h.s. vector in (3.8), the ambient strain was modified to account for the deformation of the sphere: $\mathbf{E}_{1}-\mathbf{E}_{\infty}$ and $\mathbf{E}_{2}-\mathbf{E}_{\infty}$.) In short, the steps required to solve the problem were as follows: transformation to cylindrical coordinates with the line of centers being the $z$-axis, defining stretched variables, and expanding the velocity components and pressure in powers of the nondimensional gap $\varepsilon$. In order to apply boundary conditions the equations for sphere surfaces were expanded in powers of $\varepsilon$ as well.

Coefficients $\mathrm{G}, \mathrm{H}$ and $\mathrm{M}$ in the grand matrix for close separation were estimated with the help of the asymptotic method (see Jeffrey [1992].)

\subsection{Molecular Effects}

In the Stokesiarl formulation, there is a barrier which prevents drops from colliding. This is not in accord with the physical reality because the assumption of the fluid continuity is not valid when the gap between drops is of the order of the molecular free path. (Note that for the atmosphere the molecular free path is of the order of $0.1 \mu \mathrm{m}$ for typical clouds altitudes.) No attempt is known to the author to solve the collision problem from the molecular point of view. On the other hand, some thoughts have been given to incorporate molecular properties into the hydrodynamic treatment. In particular, the so called slip-flow theory was introduced [Davis, 1972; Jonas, 1972].

The slip-flow theory assumes that only the velocity component normal to the surface of the body is equal that of the body surface. The difference of tangential components of the velocity of the body and of the fluid at the surface of the body is proportional to the local tangential stress in the fluid. This velocity difference is 
called fluid slip. Schematically, it can be written as

$$
\beta\left(v_{\xi}-v_{\xi}^{0}\right)= \pm \tau_{\eta \xi}
$$

where $\eta$ and $\xi$ are the bispherical coordinates ( $\eta$ is constant at the surfaces of the sphere), and the sign is positive for one drop and negative for the other. $\beta$ is the coefficient of external friction. The comparison of the experimental [Brown et al., 1946] and theoretical [Lamb, 1932] results for capillary flows resulted in the following expression

$$
\frac{\mu}{\beta}=1.5 \lambda
$$

where $\lambda$ is the mean free path. Davis [1972] used the above expression to calculate hydrodynamic forces for the simplest case of motion along the line of centres. His calculations shows a substantial decrease of hydrodynamic forces compared to no-slip calculations. No other related work is known to us. 


\section{4}

\section{Modelling \\ of the Turbulent Velocity Field}

Turbulence modelling has always been both a very active and a very controversial scientific subject. Yet the modelling results are seldom satisfactory. In this chapter, we present our motivation behind the choice of the method with which we simulate the turbulent velocity field as well as the method itself. Also, we describe some statistical tests which were performed with the obtained velocity field.

\subsection{Considering a Method}

In a significant number of instances, the knowledge of statistical properties of a turbulent velocity field alone is insufficient for modelling purposes. These are situations involving movements of particles or bubbles suspended in fluids as well as flows of chemically reacting fluids. In particular, the treatment of the relative motion of interacting droplets, an inherently nonisotropic process, is not easy without modelling the evolving instantaneous velocity field. (At least, this has not been successfully accomplished so far.) According to the current state-of-the-art knowledge, small-scale turbulent velocity fields which are believed to have an influence on the relative motion of interacting droplets, can be modelled either by the direct numerical simulation or by the method of random Fourier modes [Fung et al., 1992]. 
The direct numerical simulation (DNS) amounts to a numerical solution of the time-dependent Navier-Stokes equations. With more and more powerful computers, direct simulations have become possible. (The first three-dimensional simulation was performed by Orszag \& Patterson [1972].) For the Reynolds number based on the integral scale of atmospheric turbulence, the number of degrees of freedom is $R e^{\frac{9}{4}}$ (three-dimensional flow). Resolutions that high are not possible with the current size of computers, and will not be possible in the near future. At present, spectral methods used in DNS's have, at maximum, $846^{3}$ degrees of freedom [Lesieur, 1990, p. 317]. Consequently, high Reynolds number simulations are impossible. Intrinsically, DNS's are not able to reproduce the pure inertial (zero dissipation) flow as this would require an infinite number of degrees of freedom. Still, the comparison of the results produced by DNS's with experimental data is definitely encouraging, especially for smaller scales. With the appropriate external forcing, Kerr [1990] was even able to reproduce $k^{-\frac{5}{3}}$ spectrum for a substantial period of time (though for a moderate Reynolds number, 86 in particular). With all its limitations, the DNS approach seems to be suitable for investigating particle dispersion (see for example Riley \& Patterson [1974]). However, the computational requirements (both speed and memory) are much beyond what was available for the present research. Thus, we direct our attention to the other previously mentioned method.

The method of random Fourier modes was introduced by Kraichnan [1970] as a tool with which to examine diffusion of particles. This very convenient method represents a turbulent velocity field in the form of a series of random Fourier modes. The 'randomness' of the modes arises from generating the wavenumbers, frequencies, and amplitudes of the Fourier modes with the help of selected random numbers. More specifically, these random numbers are filtered and scaled in such a way that the flow is incompressible, and a specific kinetic energy spectrum, $E(k)$ is realized. The 
original method was later used and modified by a number of authors. Maxey [1987] presented an elegant extension to the method allowing application to an arbitrary energy spectrum. Fung et al. [1992] modified the method to include not only the wavenumber but also the frequency spectrum. Here, the user-friendly formalism of Maxey [1987] will be used, enriched, however, by the representation of the frequency spectrum as in Fung et al. [1992]. Before proceeding with the actual method of turbulence generation, a brief summary of some statistical properties of turbulence is in order.

\subsection{Some Aspects of Statistical Description of Iso- tropic Turbulence}

We make no attempt to present a comprehensive review of various aspects of statistical theories of turbulence. Rather only the basic ideas and formulac, crucial for the understanding of subsequent sections, are briefly summarized. For more in-depth analyses consult Batchelor [1953], Panchev [1971], or Monin \& Yagloin [1975].

Let us recall once again the basic assumptions: turbulence is homogencous, isotropic and stationary; also, the flow is incompressible. The four-dimensional (space-time) two-point correlation function for a velocity field is defined as

$$
R_{i j}(\mathbf{r}, \tau)=\overline{u_{i}(\mathbf{x}, t) u_{j}(\mathbf{x}+\mathbf{r}, t+\tau)}
$$

where $u_{i}(\mathbf{x}, t)$ are the velocity components and the overline represents the cnsemble average. For multidimensional random processes, the following theorem holds [Cramér, 1940]: The necessary and sufficient condition that $R_{i j}(r, \tau)$ be the correlation tensor is that it can be expressed in the form

$$
R_{i j}(\mathbf{r}, \tau)=\iiint_{-\infty}^{\infty} d^{3} \mathbf{k} \int_{-\infty}^{\infty} d \omega \Phi_{i j}(\mathbf{k}, \omega) e^{i(\mathbf{k} \cdot \mathbf{r}+\omega \tau)}
$$


where $\Phi_{i j}(\mathbf{k}, \omega)$ is the tensor, called the space-time spectrum such that

$$
\iiint_{-\infty}^{\infty} d^{3} \mathrm{k} \int_{-\infty}^{\infty} d \omega \Phi_{i j}(\mathrm{k}, \omega)<\infty, \quad \Phi=X_{i} X_{j} \Phi_{i j}(\mathrm{k}, \omega)
$$

and $\Phi \geq 0$ for an arbitrary choice of complex constants $X_{i}$. Clearly,

$$
\Phi_{i j}(\mathbf{k}, \omega)=\frac{1}{(2 \pi)^{4}} \iiint_{-\infty}^{\infty} d \mathbf{r} \int_{-\infty}^{\infty} d \tau R_{i j}(\mathbf{r}, \tau) e^{-i(\mathbf{k} \cdot \mathbf{r}+\omega \tau)}
$$

$\Phi_{i j}(\mathbf{k}, \omega)$ is an isotropic second order tensor and thus can be expressed

$$
\Phi_{i j}(\mathbf{k}, \omega)=A(k, \omega) k_{i} k_{j}+B(k, \omega) \delta_{i j}
$$

Further, the continuity equation for incompressible flow yields

$$
\frac{\partial}{\partial r_{i}} R_{i j}(\mathrm{r}, \tau)=0 \quad \text { and } \quad \frac{\partial}{\partial r_{j}} R_{i j}(\mathrm{r}, \tau)=0 .
$$

Substituting for $R_{i j}(\mathbf{r}, \tau)$ its spectral representation (4.2), gives the following

$$
k_{j} \Phi_{i j}(\mathbf{k}, \omega)=k_{i} \Phi_{i j}(\mathbf{k}, \omega)=0
$$

Multiplying (4.5) by $k_{i}$ and using (4.7) results in the condition

$$
A(k, \omega) k^{2}=-B(k, \omega)
$$

The spectrum can now be written in the form

$$
\Phi_{i j}(\mathbf{k}, \omega)=A(k, \omega)\left(k_{i} k_{i j}-k^{2} \delta_{i j}\right)
$$

or formally,

$$
\Phi_{i j}(\mathbf{k}, \omega)=\phi(k, \omega)\left(\delta_{i j}-\frac{k_{i} k_{j}}{k^{2}}\right)
$$

The turbulent kinetic energy spectrum is probably the most commonly used spectral function. In four-dimensional space, it is defined as

$$
\mathcal{E}(k, \omega)=\frac{1}{2} \int_{|\mathbf{k}|=k} \Phi_{i i}(\mathbf{k}, \omega) d S(\mathbf{k})
$$


Then, the average turbulent kinetic energy is calculated as

$$
\frac{q^{2}}{2}=\int_{0}^{\infty} d k \int_{-\infty}^{+\infty} \varepsilon(k, \omega) d \omega
$$

where $q$ is the r.m.s. velocity. Substituting (4.10) into (4.11) we obtain

$$
\mathcal{E}(k, \omega)=4 \pi k^{2} \phi(k, \omega)
$$

For the three-dimensional spectrum, $\Psi_{i j}(\mathbf{k})$

$$
\Psi_{i j}(\mathbf{k})=\frac{1}{(2 \pi)^{3}} \iiint_{-\infty}^{\infty} \overline{u_{i}(\mathbf{x}, t) u_{j}(\mathbf{x}+\mathbf{r}, t)} e^{-i \mathbf{k} \cdot \mathbf{r}} d^{3} \mathbf{r}
$$

the energy spectrum, $E(k)$ is defined as

$$
E(k)=\frac{1}{2} \int_{|\mathbf{k}|=k} \Psi_{i i}(\mathbf{k}) d S(\mathbf{k}) .
$$

Also, the following relations between quantities defined in the three- and four-dimensional space can be identified

$$
E(k)=\int_{-\infty}^{\infty} \mathcal{E}(k, \omega) d \omega \quad \text { and } \quad \Psi_{i j}(\mathbf{k})=\int_{-\infty}^{\infty} \Phi_{i j}(\mathbf{k}, \omega) d \omega
$$

Finally, we introduce the time spectra: Eulerian, $\chi_{i j}(\omega)$

$$
\chi_{i j}(\omega)=\frac{1}{2 \pi} \int_{-\infty}^{\infty} e^{-i \omega \tau} R_{i j}(\tau) d \tau
$$

where

$$
\overline{u_{i}(\mathbf{x}, t) u_{j}(\mathbf{x}, t+\tau)}=R_{i j}(\tau)=\int_{-\infty}^{\infty} e^{i \omega \tau} \chi_{i j}(\dot{\omega}) d \omega
$$

and Lagrangian, $\vartheta_{i j}(\omega)$

$$
\vartheta_{i j}(\omega)=\frac{1}{2 \pi} \int_{-\infty}^{\infty} e^{-i \omega \tau} \mathcal{R}_{i j}(\tau) d \tau
$$

where

$$
\overline{v_{i}(\mathbf{a}, t) v_{j}(\mathbf{a}, t+\tau)}=\mathcal{R}_{i j}(\tau)=\int_{-\infty}^{\infty} e^{i \omega \tau} \vartheta_{i j}(\omega) d \omega
$$


In the latter expression, $v_{i}(\mathbf{a}, t)$ represents the velocity of a fluid particle (Lagrangian velocity), and $\mathbf{a}$ is the initial position of the fluid particle. Again, $\chi_{i j}(\omega)$ can be obtained by integration of $\Phi_{i j}(k, \omega)$

$$
\chi_{i j}(\omega)=\iiint_{-\infty}^{\infty} \Phi_{i j}(\mathbf{k}, \omega) d^{3} \mathbf{k}
$$

Note that similarly to the energy spectrum, $E(k)$, the trace of $\chi_{i j}(\omega)$ integrates to the total kinetic energy

$$
\frac{q^{2}}{2}=\frac{1}{2} \int_{-\infty}^{\infty} \chi_{i i}(\omega) d \omega
$$

Later, our attention will be dedicated to the energy spectrum, $E(k)$ and the time spectrum, $\chi_{i i}$ because these spectra are frequently modelled and verified experimentally.

\subsection{Energy Spectra}

As the turbulent energy cascades from large to small scales, the anisotropy characterizing larger scales disappears. The small scales are therefore homogeneous, isotropic, and statistically stationary. This idea was for the first time introduced by Kolmogorov [1941] who termed such a small-scale velocity field as locally isotropic. The concept of local isotropy is narrower than that of isotropy introduced by Taylor [1934]. The locally isotropic flow is also statistically stationary, and the restrictions defining isotropy are imposed only on the distribution laws of differences of velocities (not on the distribution laws of velocities themselves.) As a consequence, for high Reynolds number flows, the energy spectrum is distinctively different for small and large wavenumbers. Kolmogorov's first similarity hypothesis states that the smallscale motion, if scaled by $\eta_{K}$ and $v_{K}$ (the Kolmogorov length and velocity respectively), dependi only on the energy dissipation rate and the fluid kinematic viscosity:

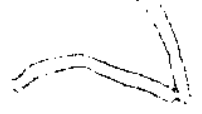


$E(k)=E(k ; \epsilon, \nu)$. (This is the, so called, equilibrium or universal range.) While Kolmogorov's second similarity hypothesis assumes that for scales large in comparison to $\eta_{K}$, the viscosity of the fluid has practically no effect, and $E(k)=E(k ; \epsilon)$. (This defines the inertial subrange.) Scale analysis of the problem yields

$$
E(k)=\alpha \epsilon^{\frac{2}{3}} k^{-\frac{5}{3}} \text {. }
$$

(Nota bene the above expression appeared for the first time in the papers by Obukhov $[1949 \mathrm{a}, \mathrm{b}]$.$) The inertial subrange exists only for large Reynolds numbers. In the case$ of large energy containing eddies it is expected that $E(k)=E(k ; \epsilon, S)$, where $S$ is the average strain of the flow, and the spectrum differs from one flow type to another. (For the details of the scale analysis see Tennekes \& Lumley [1980], pp. 262-265.)

While in the inertial subrange energy is merely transported from larger to smaller scales, most of the dissipation takes place in the vicinity of the wavenumber $k=1 / \eta_{K}$, in the, so called, dissipation subrange. The form of the energy spectrum in the entire equilibrium range has not been established successfully (in the dissipation subrange in particular). Because in the dissipation subrange the similarity hypotheses were of little use, spectral energy-transfer hypotheses were introduced. Let us start with the dynamic equation for the correlation tensor, $R_{i j}(\mathrm{r}, \tau)$. This can be directly derived from the Navier-Stokes equation, and, for the general case of a time-dependent process, has the form

$$
\frac{\partial R_{i j}(\mathbf{r}, t)}{\partial t}=\mathcal{T}_{i j}(\mathbf{r}, t)+\mathcal{P}_{i j}(\mathbf{r}, t)+2 \nu R_{i j}(\mathbf{r}, t)
$$

where

$$
\mathcal{T}_{i j}(\mathrm{r}, t)=\frac{\partial}{\partial r_{k}}\left(\overline{u_{i} u_{k}^{\prime} u_{j}^{\prime}}-\overline{u_{i} u_{k} u_{j}^{\prime}}\right) \quad \text { and } \quad \mathcal{P}_{i j}(\mathrm{r}, t)=\frac{1}{\rho}\left(\frac{\partial \overline{p u_{j}^{\prime}}}{\partial r_{i}}-\frac{\partial \overline{p u_{i}^{\prime}}}{\partial r_{j}}\right) \text {. }
$$

Primes denote field variables taken at $x+r$ as opposed to those taken at, $x$. The application of a Fourier transform in the form $\frac{1}{(2 \pi)^{3}} \iiint_{-\infty}^{\infty} e^{-i \mathbf{k} \cdot \mathbf{r}} d^{3} \mathbf{r}$ to $(4.24)$, and, 
subsequently, the condition of isotropic turbulence lead to

$$
\frac{\partial E(k, t)}{\partial t}=T(k, t)-2 \nu k^{2} E(k, t)
$$

In the above expression, $T(k, t)$ represents the Fourier transform of $\mathcal{T}_{i j}(\mathbf{r}, t)$ and its more detailed form will not be introduced here. (For details see Monin \& Yaglom [1975], p. 123.) The Fourie transform of $\mathcal{P}_{i j}(\mathbf{r}, t)$ vanishes for isotropic turbulence. Now let us integrate the spectral equation (4.26)

$$
\begin{gathered}
\frac{\partial}{\partial t} \underbrace{\int_{0}^{k} E\left(k^{\prime}, t\right) d k^{\prime}}_{\text {I }}=-\underbrace{W(k, t)}_{\text {II }}-\underbrace{2 \nu \int_{0}^{k} k^{2} E\left(k^{\prime}, t\right) d k^{\prime}}_{\text {III }}, \\
\frac{\partial}{\partial t} \underbrace{\int_{k}^{\infty} E\left(k^{\prime}, t\right) d k^{\prime}}_{\text {IV }}=\underbrace{W(k, t)}_{\text {II }}-\underbrace{2 \nu \int_{k}^{\infty} k^{2} E\left(k^{\prime}, t\right) d k^{\prime}}_{\text {V }},
\end{gathered}
$$

where the physical meaning of the terms is as follows:

I. Energy associated with macro scales.

II. Rate of energy transfer from macro to micro scales.

III. Energy dissipation rate of macro scales.

IV. Energy associated with micro scales.

V. Energy dissipation rate of micro scales.

$W(k, t)=\int_{k}^{\infty} T\left(k^{\prime}, t\right) d k^{\prime}$ is the unknown rate of transfer of energy through the wavenumber $k$. Many hypotheses have been introduced in order to model the timeindependent rate of energy transfer, $W(k)$ : Obukhov [1941], Kovasznay [1948], Heisenberg [1948], Pao [1965], and others. All of them have some flaws, the discussion of which is beyond scope of this review. A thorough discussion of these hypotheses can be found in Monin \& Yaglom [1975], pp. 212-235. Here, the Pao hypothesis will be presented because of reasons which become clear later.

For a statistically stationary process, Pao assumes the following form of the rate of energy transfer in the universal range (micro scales)

$$
W(k)=\sigma(k) E(k)
$$


where $\sigma$ is derived using dimensional reasoning as

$$
\sigma(k)=\alpha^{-1} \epsilon^{\frac{1}{3}} h^{\frac{5}{3}}
$$

$\alpha$ being a constant. Substituting (4.29) and (4.30) into the differential equation

$$
-\frac{d W(k)}{d k}=T(k)=2 v k^{2} E(k)
$$

and, then, solving it for $E(k)$ gives

$$
E(k)=A k^{-\frac{5}{3}} \exp \left(-\frac{3}{2} \alpha \nu \epsilon^{-\frac{1}{3}} k^{\frac{4}{3}}\right) \text {. }
$$

An approximate value of the constant $A$ is estimated by substituting the above expression into

$$
\epsilon=2 \nu \int_{0}^{\infty} k^{2} E(k) d k
$$

The final form of the spectrum is

$$
E(k)=\alpha \epsilon^{\frac{2}{3}} k^{-\frac{5}{3}} \exp \left(-\frac{3}{2} \alpha \nu \epsilon^{-\frac{1}{3}} k^{\frac{4}{3}}\right) .
$$

Measurements show that constant $\alpha$ is approximately equal to 1.5 [Grant et al., 1962; Gibson, 1963]. Spectrum (4.34) reduces to the $-\frac{5}{3}$ law, (4.23) for small wavenumbers.

Plenty of experiments have been conducted in order to verify Kolmogorov's hypotheses as well as various forms of the energy spectrum. There is a general consensus that in the majority of flow types both of Kolmogorov's hypotheses are true or, at. least, provide a good first order approximation (see for example Mestayer [1982]). The experimental results overwhelmingly support the $-\frac{5}{3}$ law in both experimental settings and geophysical flows. The form of the energy spectrum in the dissipation subrange is not well established. Instrumental limitations do not allow measurements of the spectrum below the Kolmogorov length. Most measurements of energy spectra have been performed with hot-wire or hot-film anemometers. The ability of such instruments to register small scale variations is limited by both the finite size of the 
instrument and thermal inertia of the wire [Hinze, 1975]. With the scant data available, the Pao spectrum performs better when compared with measurements than the spectra obtained by Kovasznay and Heisenberg [Pao, 1965]. The data sets used in the comparison were the energy spectra in a tidal stream [Grant et al., 1962], and a round air jet [Gibson, 1963]. The more recent data in a tidal flow [Gargett et al., 1984] are similar to those obtained by Grant et al. Because both the spectrum by Pao and the data by Gargett et al. are compared with the data by Grant et al., one can indirectly draw a conclusion that the Pao spectrum is in even better agreement with the data by Gargett et al. than those by Grant et al. Still, one should bear in mind that all the comparisons are made with a very limited and poor data pool.

\subsection{Time Spectra}

A transplantation of Kolmogorov's similarity ideas into the frequency domain lead to the derivation of an expression for the Lagrangian frequency (time) spectrum [Inoue, 1951]. Using purely dimensional reasoning he obtained for the inertial subrange

$$
\vartheta_{11}(\omega)=\beta_{L} \epsilon \omega^{-2}
$$

where $\beta_{L}$ is a constant presumably of the order of unity. The inertial subrange is confined between the energy containing range and the dissipation subrange. The lower limit being defined by the inverse of the integral Lagrangian time scale and the upper by the Kolmogorov frequency [Corrsin, 1963]:

$$
\omega_{L}=\frac{1}{\tau_{L}}=\frac{1}{\frac{1}{q^{2}} \int_{0}^{\infty} \mathcal{R}_{i i}(\tau) d \tau} \quad \text { and } \quad \omega_{K}=\sqrt{\frac{\epsilon}{\nu}}
$$

In a similar way, the Eulerian spectrum was derived by Corrsin [1963]. However, the measurements of Comte-Bellot \& Corrsin [1971] put the Kolmogorov scaling for the 
Eulerian spectrum in question. The estimate of the Eulerian Taylor (time) microscale,

$$
\lambda_{E}^{2}=\frac{2 \overline{u_{1}^{2}}}{\int_{0}^{\infty} \omega^{2} \chi_{i i} d \omega}
$$

exceeded that supplied by the theory. This fact inspired Tennekes [1975] to seek a new theory. He calculated an estimate of the Eulerian microscale assuming that advection of small eddies by large eddies is governed by Taylor hypothesis. The important assumption made in the derivation was that micro scales are statistically independent from the energy containing eddies. The result was in accord with the formerly mentioned measurements. The form of the time spectrum derived be Tennekes is

$$
\chi_{i i}(\omega)=\beta_{E} \epsilon^{\frac{2}{3}} k^{-\frac{2}{3}} \omega^{-\frac{\pi}{3}}
$$

where $\beta_{E}$ is a constant presumably of the order of unity. This hypothesis is often called random sweeping hypothesis, and has been in the centre of controversy since it was introduced (see Nelkin [1992]). However, the experimental evidence seems to support the form of the Eulerian spectrum derived by Tennekes [Praskovsky et, al., 1993]. The lower and upper limit of the inertial subrange for the Eulerian spectrum are

$$
\omega_{E}=\frac{1}{\tau_{E}}=\frac{1}{\frac{1}{q^{2}} \int_{0}^{\infty} R_{i i}(\tau) d \tau} \quad \text { and } \quad \omega_{D}=\frac{q}{\eta_{K}},
$$

where $\tau_{E}$ is the Eulerian integral time scale.

Fortunately for us, because of the small scales which are considered here, there is no need to worry about the sweeping effect of large scales. Therefore, even if the required time spectrum is Eulerian in nature, the mathematical expression similar to the Lagrangian spectrum will be applied. In this we follow Fung ct, al. [1992] who used for such a spectrum the term Eulerian-Lagrangian spectrum. The EulerianLagrangian time spectrum takes then the form

$$
\chi_{i i}^{E L}(\omega)=\beta_{E L} \epsilon \omega^{-2}
$$


One can conclude that the small scales under consideration and the eddy-containing large scales are decorrelated for the purpose of our investigation.

\subsection{The Method of Random Fourier Modes}

In this section, we present the method of random Fourier modes as well as the modifications which apply to the problem at hand. Let the flow be represented by the series of random Fourier modes as follows [Kraichnan, 1970]

$$
u_{i}(\mathbf{x}, t)=\sum_{n=1}^{N}\left\{b_{i}^{(n)} \cos \left(\mathbf{k}^{(n)} \cdot \mathbf{x}+\omega^{(n)} t\right)+c_{i}^{(n)} \sin \left(\mathrm{k}^{(n)} \cdot \mathbf{x}+\omega^{(n)} t\right)\right\} .
$$

After Maxey [1987], let $\mathbf{k}$ and $\omega$ be random variables with the probability density functions $p_{1}(\mathrm{k})$ and $p_{2}(\omega)$ respectively. The coefficients $b_{i}^{(n)}$ and $c_{i}^{(n)}$ must be chosen in such a way that the flow is incompressible, and the two-point velocity correlation corresponds to the desired energy spectrum. (Again we followiMaxey [1987].) The condition of incompressibility

$$
\frac{\partial u_{i}}{\partial x_{i}}=0 \quad \Rightarrow \quad \mathbf{b}^{(n)} \cdot \mathbf{k}^{(n)}=\mathbf{c}^{(n)} \cdot \mathbf{k}^{(n)}=0
$$

is satisfied for coefficients of the form

$$
b_{i}^{(n)}=\Gamma(k, \omega)\left[\delta_{i j}-\frac{k_{i}^{(n)} k_{j}^{(n)}}{k^{(n) 2}}\right] \hat{b}_{j}^{(n)} .
$$

The function $\Gamma(k, \omega)$ scales the coefficients so the desired energy spectrum is obtained while the coefficients $\hat{b}_{j}^{(n)}$ are random Gaussian variables with zero mean and unit variance (similar relations hold for $\left.\hat{c}_{j}^{(n)}\right)$. For $\Gamma(k, \omega)$ to correctly represent the energy content of the modes, not only the value of the energy spectrum for a specified wavenumber and frequiency must be accounted for but also the frequency of occurrence of these modes-this is where the probability distribution functions come to work. Let the velocity correlation tensor be written in the form

$$
R_{i j}(\mathbf{r}, \tau)=\iiint_{-\infty}^{\infty} d^{3} \mathbf{k} \int_{-\infty}^{\infty} d \omega\left\{p_{1}(\mathbf{k}) p_{2}(\omega) u_{i}(\mathbf{x}, t) u_{j}(\mathbf{x}+\mathbf{r}, t+\tau)\right\}
$$


Substituting (4.41) in (4.44) results in the following expression

$$
R_{i j}(\mathbf{r}, \tau)=N \iiint_{-\infty}^{\infty} d^{3} \mathbf{k} \int_{-\infty}^{\infty} d \omega\left\{p_{1}(\mathbf{k}) p_{2}(\omega) \Gamma^{2}(k, \omega)\left[\delta_{i j}-\frac{k_{i} k_{j}}{k^{2}}\right] \cos (\mathbf{k} \cdot \mathbf{r}+\omega \tau)\right\}
$$

Equation (4.2) can be rewritten with the help of (4.10) and (4.13) as

$$
R_{i j}(\mathbf{r}, \tau)=\iiint_{-\infty}^{\infty} d^{3} \mathbf{k} \int_{-\infty}^{\infty} d \omega\left\{\frac{1}{4 \pi k^{2}} \mathcal{E}(k, \omega)\left[\delta_{i j}-\frac{k_{i} k_{j}}{k^{2}}\right] \cos (\mathbf{k} \cdot \mathbf{r}+\omega \tau)\right\}
$$

Finally, the comparison of (4.45) and (4.46) gives the scaling function $\Gamma(k, \omega)$

$$
\Gamma^{2}(k, \omega)=\frac{\mathcal{E}(k, \omega)}{4 \pi k^{2} N p_{1}(\mathbf{k}) p_{2}(\omega)}
$$

In order to obtain the expression for $\mathcal{E}(k, \omega)$, phenomenological thinking was applicu' by Fung et al. [1992]. We shall follow their path. They assume that frequency is spread in a Gaussian manner with the variance $\sigma_{\omega}^{2}$ around the characteristic frequency $\bar{\omega}$. (Both parameters of the distribution are functions of $k$.) The spectrum $\mathcal{E}(k, \omega)$ is then

$$
\mathcal{E}(k, \omega)=E(k) \frac{1}{\sqrt{2 \pi} \sigma_{\omega}} \exp \left(-\frac{(\omega-\bar{\omega})^{2}}{2 \sigma_{\omega}^{2}}\right) .
$$

Fung et al. [1992] assume after Leslie [1973] that

$$
\sigma_{\omega}=\bar{\omega}=\epsilon^{\frac{1}{3}} k^{\frac{2}{3}}
$$

The numerical calculation of Fung et al. shows that the above choice of $\mathcal{E}(k, \omega)$ results in the Eulerian-Lagrangian spectrum (4.40). Finally, let us recall that $E(k)$ has the form of the Pao spectrum.

Equation (4.47) will now be complete if the wavenumber and frequency probability density functions are specified. The problem at hand requires relatively wide spectral bands. This suggests that all three wavenumber components, and the frequency be distributed with rectangular distributions in their respective ranges: $\left(k_{l} \leq k_{i} \leq k_{0}\right.$; $\left.-k_{0} \leq k_{i} \leq-k_{l}\right)$ and $\left(\omega_{l} \leq \omega \leq \omega_{0} ;-\omega_{0} \leq \omega \leq-\omega_{l}\right)$. While the magnitude of $\omega_{0}$ 
is naturally determined by the viscous cutoff (the inverse of the Kolmogorov time scale), the determination of the magnitude of $k_{0}$ calls for a more laborious approach. With higher and higher wavenumbers the amplitude of the motion as well as of the velocity gradient become smaller and smaller. Both criteria can be used to perform the truncation of high wavenumbers. In the wavenumber space, the amplitude of the motion is represented by the total kinetic energy, $\int E(k) d k$, and the velocity gradient by the dissipation, $\int k^{2} E(k) d k$. Thus, the following criteria were obtained

$$
\begin{gathered}
\frac{\int_{k_{*}}^{k_{o f f}} E(k) d k}{\int_{k_{*}}^{\infty} E(k) d k}=1-\delta, \\
\frac{\int_{k_{*}}^{k_{o f f}} k^{2} E(k) d k}{\int_{k_{*}}^{\infty} k^{2} E(k) d k}=1-\delta,
\end{gathered}
$$

where $k_{*}$ is the lower limit of the universal range and $\delta$ is a small number. Both criteria agree well, giving a value of $k_{\text {off }}$ of the order of $\frac{2}{\eta_{K}}$ for $\delta=0.001 . k_{0}$, then, must be chosen either equal or somewhat smaller than $k_{\text {off }}$. Note that both spectra are valid only in their respective universal ranges, lower limits of which are determined by the respective values of the energy containing eddies, $k_{*}$ and $\omega_{*}$. These, in turn, are associated with the integral scales of turbulence. We obtain crude estimates of the above scales on the basis of dimensional thinking as follows

$$
k_{*}=\frac{1}{l_{*}}=\frac{\epsilon}{q^{3}} \quad \text { and } \quad \omega_{*}=\frac{1}{t_{*}}=\frac{q}{l}=\frac{\epsilon}{q^{2}}
$$

where $l_{*}$ and $t_{*}$ are the integral scales and represent the length and time scales characterizing the energy containing eddies. $\omega_{l}$ shäll be later identified with $\omega_{*}$, and $k_{l}$ with $k_{*} / \sqrt{3}$. For a developing cumulus cloud, the r.m.s. value of the velocity, $q$ is assumed to be between 1 and $10 \mathrm{~m} \mathrm{sec}^{-1}$ (based on the measurements by MacPherson [1979].) The model is insensitive to the choice of $k_{*}$ and $\omega_{*}$. Thus, the choice of $q$ is not very important. ( $k_{*}$ and $\omega_{*}$ are at least $10^{4}$ times smaller than $k_{0}$ and $\omega_{0}$ ) In practice, some other truncations were introduced; these, however, were determined

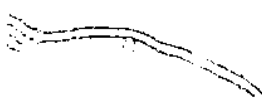


by the drop characteristic scales, and, therefore, are presented together with the detailed description of the calculation of collision kernels. Nevertheless, in the following section, some aspects of computer generation of the turbulent field as well as some obtained statistics are discussed.

\subsection{Realizations of the Turbulent Field}

Before employing the turbulence model to examine collisions of drops, we examined whether or not the generated turbulent field is robust. After making the appropriate substitutions for $\mathcal{E}(k, \omega)$ : (4.48), $E(k)$ : (4.34), and the probability density functions

$$
p_{1}(k)=\frac{1}{\left(2 k_{0}\right)^{3}} \quad \text { and } \quad p_{2}(\omega)=\frac{1}{2 \omega_{0}}
$$

the equation for the scaling function $\Gamma(k, \omega)$ is

$$
\Gamma^{2}(k, \omega)=\frac{4}{\sqrt{2} \pi^{\frac{3}{2}}} \frac{\alpha k_{0}^{3} \omega_{0} \epsilon^{\frac{2}{3}}}{N} \epsilon^{\frac{1}{3}} k^{-\frac{4}{3}} \exp \left(-\frac{3}{2} \alpha \nu \epsilon^{-\frac{1}{3}} k^{\frac{4}{3}}\right) \exp \left(-\frac{\left(\omega-\epsilon^{\frac{1}{3}} k^{\frac{2}{3}}\right)^{2}}{2 \epsilon^{\frac{2}{3}} k^{4}}\right) .
$$

Note that in (4.53), it was assumed that $k_{l}$ and $\omega_{l}$ are much smaller than $k_{0}$ and $\omega_{0}$, and thus $k_{l}=\omega_{l} \approx 0$. The calculation presented here were performed for $\epsilon=10 \mathrm{~cm}^{2}$ $\sec ^{-3}$. The parameters characterizing the probability distribution furictions $p_{1}(k)$ and $p_{2}(\omega)$ are chosen according to the discussion in the previous section. $\omega_{0}$ is the viscous cutoff of the frequency spectrum, and, as it was described in Section 4.4, is equal to the inverse of the Kolmogorov time scale. In its nondimensional form, $\tilde{\omega}_{n}=1$. Nondimensional $\tilde{k}_{\text {off }}=k_{\text {off }} \eta_{K}=3$, and, consequently, we choose $\tilde{k}_{0}=k_{0} \eta_{K}=1.8$. (Note that the r.m.s. value of $k$ is equal $\sqrt{3} \sqrt{\overline{k_{r}^{2}}}$.) The respective wavenumber and frequency of the energy containing eddies which constitute the lower limits of the spectra are (again nondimensional) $\tilde{k}_{*}=k_{*} \eta_{K}=10^{-6}$ and $\tilde{\omega}_{*}=\omega_{*} \eta_{K}=10^{-4}$. The most troublesome parameter to be determined in (4.54) is the number of Fourier modes. Obviously, the more Fourier modes the better the representation but also 
the longer the simulation time. Tests showed that the values of collision efficiency are slightly different for $N$ of the order of 100 compared to the values for $N$ of the order of 1000 . No tests were performed with $N$ of the order of 10,000 because of long calculation times. With this in mind we chose $N=2000$.
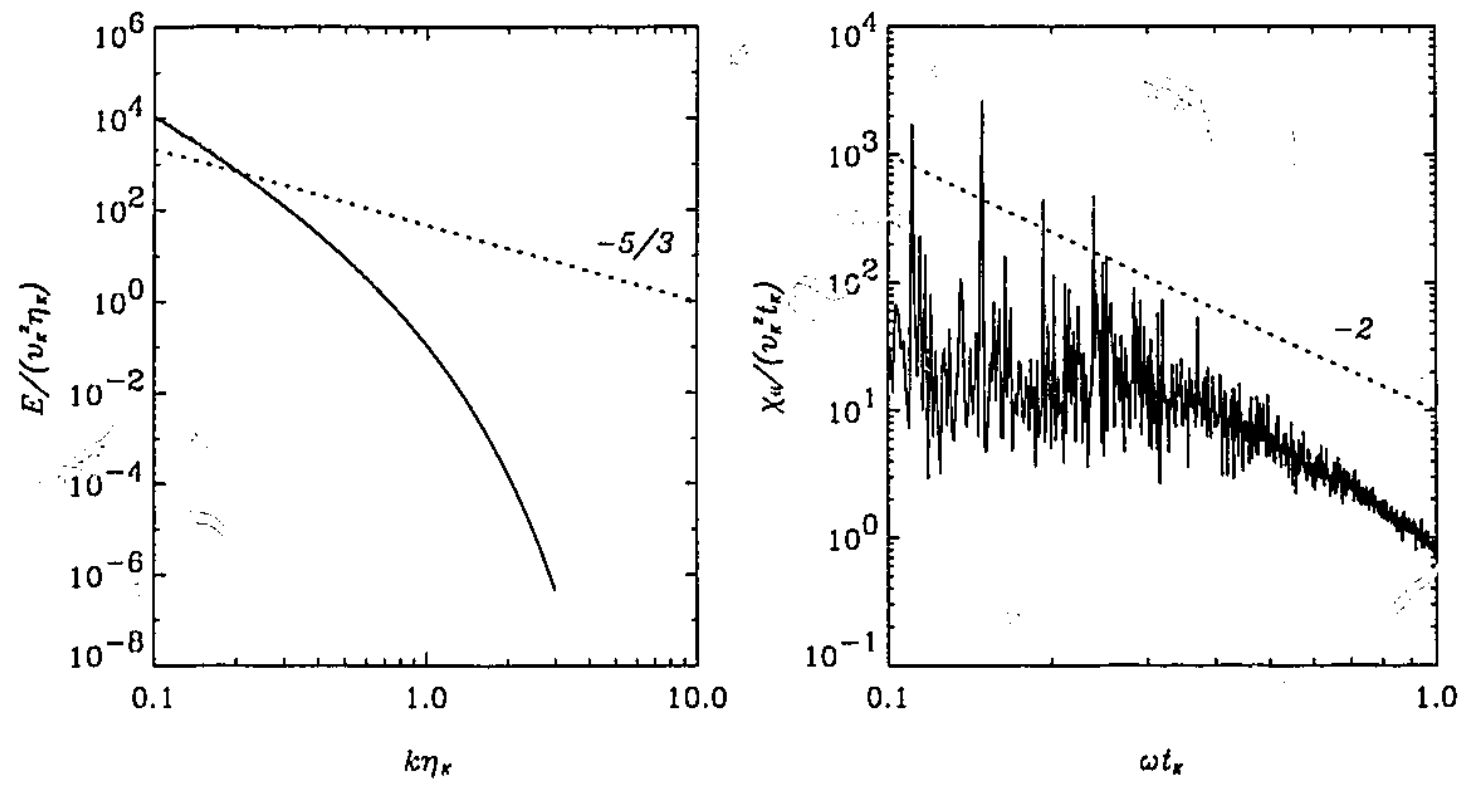

Fig. 4.1 The nondimensional wavenumler $(E)$ and frequency $\left(\chi_{i i}\right)$ spectra.

Two types of tests were performed with the turbulent field. They involved calculations of: 1 . the average spectra, and 2 . the two-point velocity correlations. The average space and time spectra for 20,000 realizations of the turbulent field are presented in Fig. 4.1. Both figures are plotted on a log-log scale. The appropriate slopes (i.e. $-5 / 3$ for the wavenumber spectrum and -2 for the frequency spectrum) are included for comparisons. The range of wavenumbers consist of the pure dissipation subrange as well as the transition region between the inertial and dissipation subranges. No pure inertial range is modelled. Therefore, the spectrum is steeper than 
$-5 / 3$ slope. The time spectrun captures relatively well the -2 slope. Also, for smaller frequencies, the tendency of the time spictrum to follow the less stcep $-5 / 3$ slope is visible. (This is due to the sweeping eifec" of larger eddies.) The only worrisome fact is the noisy character of the time spectrum. The reason is intrinsically built in the method of modelling of the time spectrum (a Gaussian distribution of frequencies for each wavenumber.) Probably, including more Fourier modes could help to obtain less noisy time spectra. This, however, was not investigated. Finally, a crude estimate of $\beta_{E L}$ in (4.40) was obtained - this, as expected, was of the order of unity, specifically 0.8 .

The second test was designed to verify the two-point velocity correlations. With the correct spectrum embedded in our model, we expect that, the velocity correlation ought to give us a reasonable agreement for the two situations $k \ll \eta_{K}$ and $k_{i} \gg \eta_{K}$. (Although, the working conditions of our model are near the Kolmogorov length scale, there are no theoretical expressions for the velocity correlation in the transitional period of interest.) Let us define the longitudinal $\left(B_{r r}\right)$ and transverse $\left(B_{n n}\right)$ velocity correlations between two points joined by vector $\mathbf{r}$ as

$$
\begin{aligned}
& B_{r r}=\overline{\left[u_{r}(\mathbf{x}+\mathbf{r})-u_{r}(\mathbf{x})\right]^{2}} \\
& B_{n n}=\overline{\left[u_{n}(\mathbf{x}+\mathbf{r})-u_{n}(\mathbf{x})\right]^{2}}
\end{aligned}
$$

where $u_{r}$ and $u_{n}$ are the longitudinal and transverse components of the velocity. According to Landau \& Lifshitz [1959], the following expressions hold in the respective pure inertial and pure dissipation regions

$$
\begin{aligned}
B_{r r}=C_{I} \epsilon^{\frac{2}{2}} r^{\frac{2}{3}} & B_{n n}=\frac{4}{3} C_{I} \epsilon^{\frac{2}{3} r^{2}} \\
B_{r r}=C_{D} \frac{\epsilon r^{2}}{\nu} & B_{n n}=2 C_{D} \frac{\epsilon r^{2}}{\nu}
\end{aligned}
$$

where $C_{I}$ is of order unity, and $C_{D}$ was calculated [Landau \& Lifshitz, 1959] as $1 / 15 \approx 0.067$. We tried to establish the lower limit of $r$ for the pure inertial region 
and the upper limit of $r$ for the pure dissipation region by calculating the ratio of the longitudinal correlation to the transverse correlation. The pure inertial range was clearly established above $50 \mathrm{~cm}$, while the pure dissipation range below $0.01 \mathrm{~cm}$. (Note that $/ \gamma_{K}$ in the test was $0.13 \mathrm{~cm}$.) The next step involved the comparison of coefficiersts $C_{I}$ and $C_{D}$ with the calculated values. The approximate values obtained in the numerical calculations were respectively $C_{I} \approx 0.9$ and $C_{D} \approx 0.056$.

We conclude that the method stands up to our expectations. The most important aspect of turbulence with respect to the process of drop collisions is its spatial variability. That aspect seems to be captured relatively well. Probably, the increase of Fourier modes by a factor of, say, five would improve the performance of the model. It will, however, increase the amount of computer time needed for calculation. 


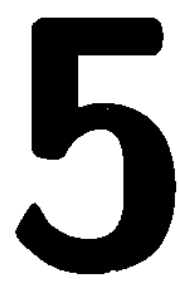

\section{Modelling Drop Trajectories}

With the tools described in Chapters 3 and 4 we are now able to model the motion of drops. In this chapter we will show the details of the model with which to obtain the trajectories. We wiil also show the examples of modelling both laminar and turbulent motion.

\subsection{The Model}

Let us recall the set of equations governing the motion of two drops. Because this is the essential part of the model to be presented, we feel at liberty to write down the equations introduced in Section 3.2 once again:

$$
\begin{gathered}
m_{\alpha} \frac{d v_{i}^{\alpha}}{d t}=F_{i}^{\alpha}+m_{\alpha} g_{i} \\
I_{\alpha} \frac{d \Omega_{i}^{\alpha}}{d t}=T_{i}^{\alpha}, \\
\frac{d x_{i}^{\alpha}}{d t}=v_{i}^{\alpha}
\end{gathered}
$$

where

$$
\begin{gathered}
F_{i}^{\alpha}=\mu A_{i j}^{\alpha \alpha}\left[u_{j}\left(\mathbf{x}^{\alpha}\right)-v_{j}^{\alpha}\right]+\mu A_{i j}^{\alpha \beta}\left[u_{j}\left(\mathbf{x}^{\beta}\right)-v_{j}^{\beta}\right]-\mu \tilde{B}_{i j}^{\alpha \alpha}\left(\Omega_{j}^{\alpha}-\Omega_{j}^{\infty}\right)-\mu \widetilde{B}_{i j}^{\alpha \beta}\left(\Omega_{j}^{\beta}-\Omega_{j}^{\infty}\right) \\
+\mu \tilde{G}_{i j k}^{\alpha \alpha} E_{j k}^{\infty}+\mu \widetilde{G}_{i j k}^{\alpha \beta} E_{j k}^{\infty}
\end{gathered}
$$




$$
\begin{gathered}
T_{i}^{\alpha}=\mu B_{i j}^{\alpha \alpha}\left[u_{j}\left(\mathbf{x}^{\alpha}-v_{j}^{\alpha}\right)\right]+\mu B_{i j}^{\alpha \beta}\left[u_{j}\left(\mathbf{x}^{\beta}-v_{j}^{\beta}\right)\right]-\mu C_{i j}^{\alpha \alpha}\left(\Omega_{j}^{\alpha}-\Omega_{j}^{\infty}\right)-\mu C_{i j}^{\alpha \beta}\left(\Omega_{j}^{\beta}-\Omega_{j}^{\infty}\right) \\
+\mu \tilde{H}_{i j k}^{\alpha \alpha} E_{j k}^{\infty}+\mu \tilde{H}_{i j k}^{\alpha \beta} E_{j k}^{\infty} .
\end{gathered}
$$

In order to proceed with the solution, the coefficients in the expressions defining forces and torques have to be determined. As well, the velocity, and the velocity gradient of the turbulent field at desired locations have to be specified.

\subsubsection{The Scalar Resistance Functions}

The coefficients $A_{i j}^{\alpha \beta}, B_{i j}^{\alpha \beta}, \widetilde{B}_{i j}^{\alpha \beta}, C_{i j}^{\alpha \beta}, \widetilde{G}_{i j}^{\alpha \beta}$, and $\tilde{H}_{i j}^{\alpha \beta}$ can be expressed using the scalar resistance functions (SRF hereafter), $X_{\alpha \beta}^{A}, Y_{\alpha \beta}^{A}, Y_{\alpha \beta}^{B}, X_{\alpha \beta}^{C}, Y_{\alpha \beta}^{C}, X_{\alpha \beta}^{G}, Y_{\alpha \beta}^{G}$, and $Y_{\alpha \beta}^{H}$ (Eqns. 3.15-3.19). As it was pointed out in Chapter 3, all the SRF's are calculated with no-slip boundary conditions. Because the evaluation of the SRF's turned out, to be a rather time consuming task, we aimed for producing convenient look-up tables.

The function $X_{\alpha \beta}^{A}$ was calculated as a superposition of the Stimson \& Jeffery [1926] solution for spheres moving with equal velocities with the modified Maud [1961] solution for spheres moving with equal speeds in opposite direct:as. The infinite series of Legendre polynomials was truncated in such a way that the truncation error was of the order of the machine roundoff error $\left(2.2 \times 10^{-16}\right.$ for the Silicon Graphics Unix work station with double precision.) The functions $Y_{\alpha \beta}^{A}, Y_{\alpha \beta}^{B}$, and $Y_{\alpha \beta}^{C}$ were obtained in quite an analogous way using the expressions of Davis [1969]. The coefficients $X_{\alpha \beta}^{C}$ were calculated as in Jeffrey \& Onishi [1984], and the coefficients $Y_{\alpha \beta}^{G}, Y_{\alpha \beta}^{H}$ as in Jeffrey [1992]. For the latter, the FORTRAN code as well as the pre-calculated coefficients were kindly given to the author by Prof. D. J. Jeffrey. All the SRF's depend only on two nondimensional parameters, the radius ratio, $\lambda$, and the parameter, $s=$ $2 D /\left(r_{1}+r_{2}\right)$, where $D$ is the distance between centres. For the sake of clarity, in the 
graphic representations, we prefer to use a slightly different parameter, $S /\left(r_{1}+r_{2}\right)$, where $S$ is the separation between the drop surfaces. In Figs 5.1-5.3, there are presented the SRF's versus $S$ for $\lambda=0.7$. Note the dramatic increase in most of the functions (especially $X_{\alpha \beta}^{A}$ i.e. coefficients associated with the motion along the line of centres) for drops in close proximity. This had some implications for the way the look-up tables were constructed, in particular, a nonuniform (logarithmic) spacing between the data points was implemented. The cubic spline interpolation was chosen as a preferred method of interpolation. In order to facilitate the search within the table all the coefficients were tabulated with the same spacing, and only one pointer was used. The effectiveness and accuracy of the interpolation sclieme was tediously tested on the calculation of laminar grazing trajectories. The adopted criterion was that the grazing trajectories obtained with both the directly calculated and the interpolated coefficients differed less than $0.1 \%$. Similar tests were also carried out for several turbulent trajectories resulting in drop collisions.

\subsubsection{Turbulent Velocity}

The method of randorn Fourier modes used here to generate the turbulent velocity field is described in Chapter 4. In this section, only the verification of the applicability of the Taylor series expansion to the turbulent velocity field, i.e. expression (3.5), is discussed. The verification applies exclusively to the length scales subsequently used in calculations which is, at maximum, of the order of 20 radii of the collector drop. Since the expansion is centred midway between the two considered drops, that scale reduces to 10 radii of the collector drop. For a $20 \mu \mathrm{m}$ collector drop this length scale is $0.02 \mathrm{~cm}$. (This is $\delta x_{j}$ in (3.5).) The question which arose was what is the value of $o\left(\delta^{2} x\right)$-is it indeed smali? We calculated the r.m.s. value of $o\left(\delta^{2} x\right) / u_{i}$ which represents the error for many realizations of the turbulent field (4000 in par- 

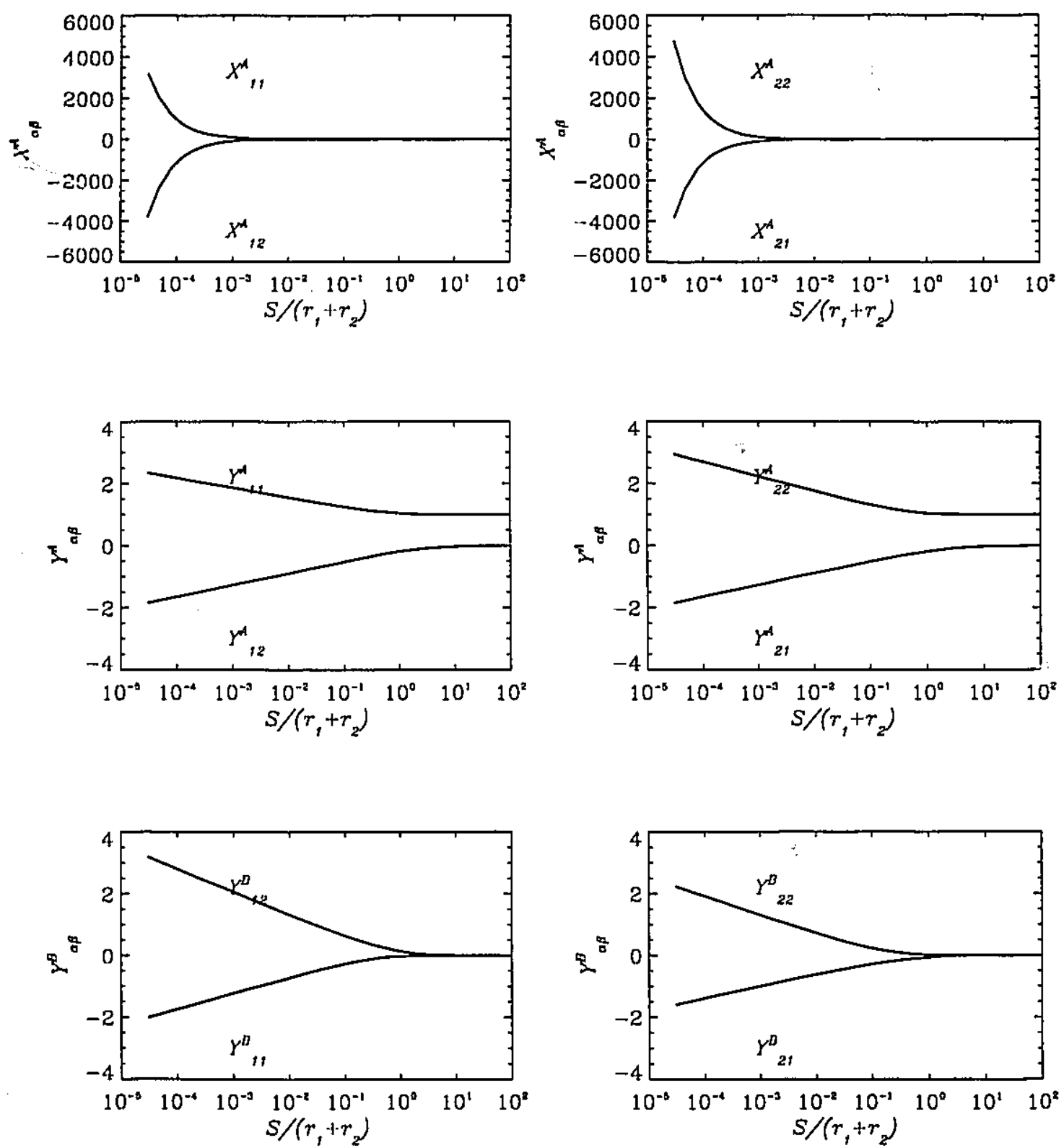

Fig. 5.1 Scalar resistance functions $X_{\alpha \beta}^{A}, Y_{\alpha \beta}^{A}$, and $Y_{\alpha \beta}^{B}$ vs. the nondimensional separation betweeii drop surfaces $S /\left(r_{1}+r_{2}\right)$ for the radii ratio $\lambda=0.7$. 

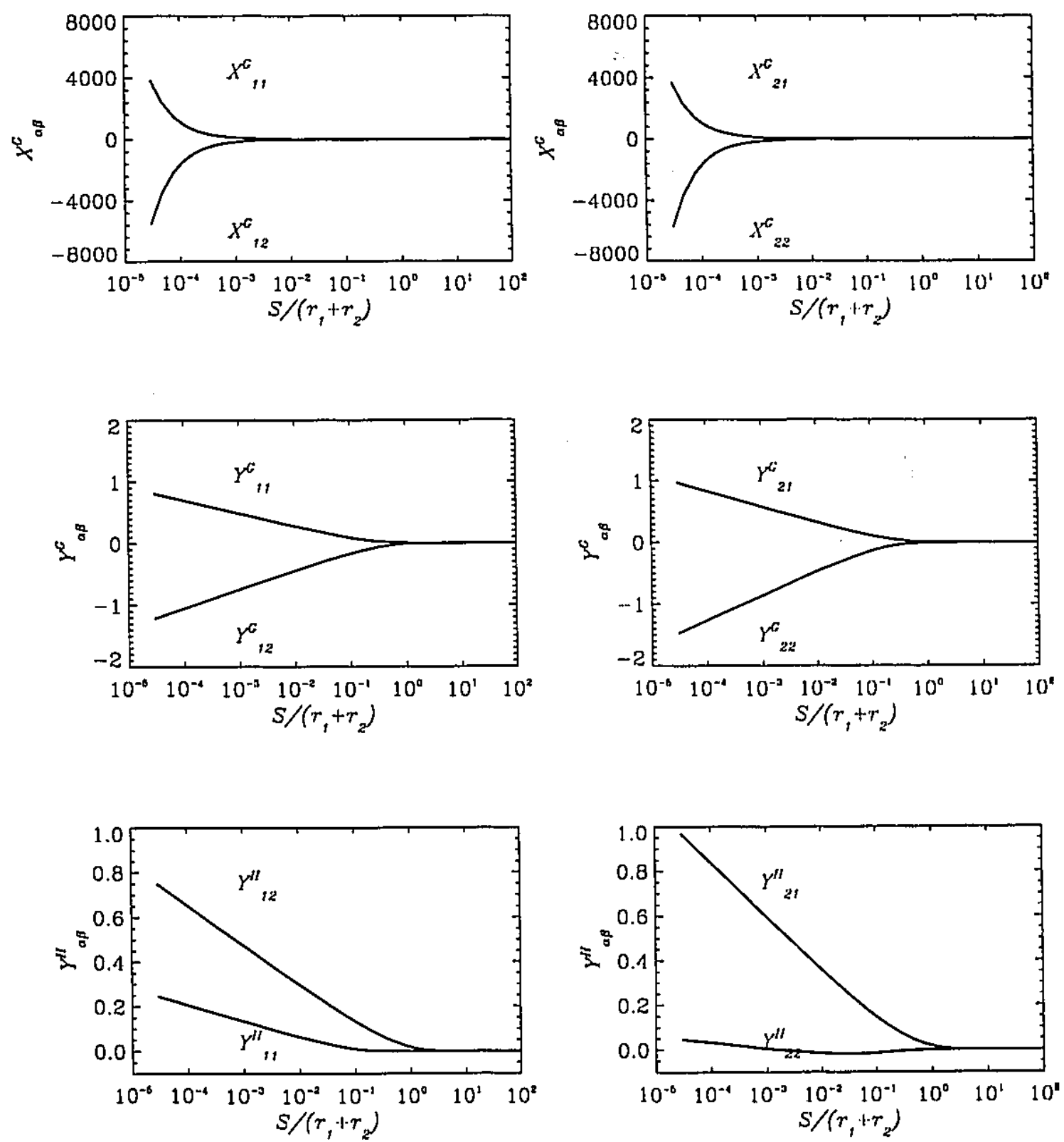

Fig. 5.2 Scalar resistance functions $X_{\alpha \beta}^{G}, Y_{\alpha \beta}^{G}$, and $Y_{\alpha \beta}^{H}$ vs. the nondimensional separation between drop surfaces $S /\left(r_{1}+r_{2}\right)$ for the radii ratio $\lambda=0.7$. 

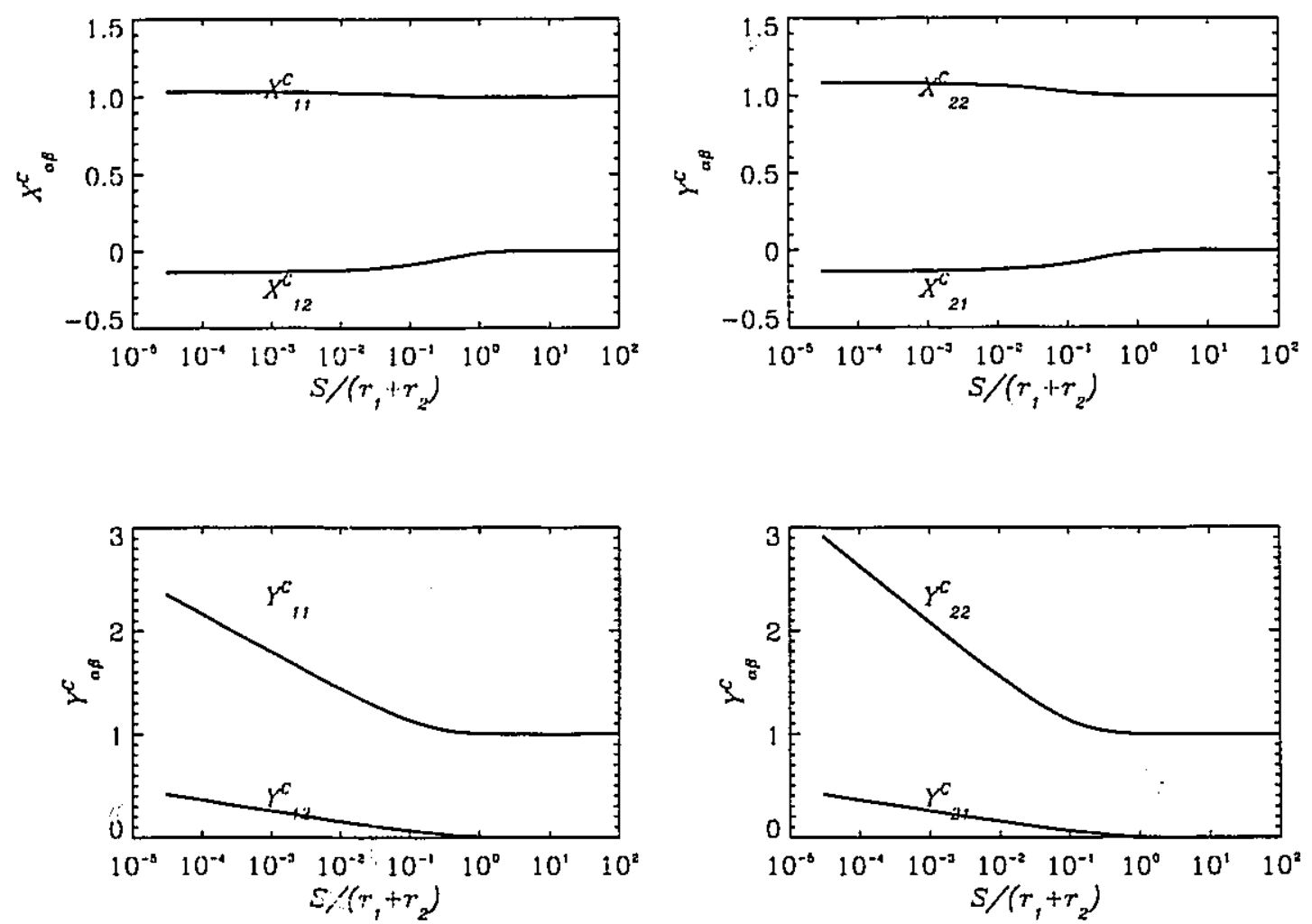

Fig. 5.3 Scalar resistance functions $X_{\alpha \beta}^{C}$, and $Y_{\alpha \beta}^{C}$ vs. the nondimensional separation between drop surfaces $S /\left(r_{1}+r_{2}\right)$ for the radii ratio $\lambda=0.7$.

ticular). For $\delta x=0.02 \mathrm{~cm}$ and $\epsilon=100 \mathrm{~cm}^{2} \mathrm{sec}^{-3}$, the error was of the order of $1 \%$. For smaller length scales and smaller rates of energy dissipation the error was smaller (e.g. for $\delta x=0.01 \mathrm{~cm}$ and $\epsilon=100 \mathrm{~cm}^{2} \mathrm{sec}^{-3}$, r.m.s. $\left[o\left(\delta^{2} x\right) / u_{i}\right]=0.1 \%$, and for $\delta x=0.02 \mathrm{~cm}$ and $\epsilon=1 \mathrm{~cm}^{2} \mathrm{sec}^{-3}$, r.m.s. $\left.\left[o\left(\delta^{2} x\right) / u_{i}\right]=0.1 \%\right)$.

Let us return to the equations governing the motion of the drops. Clearly, at each time step, it is necessary to find the velocity field at the drop positions, and the velocity gradient at the midpoint between drops. Forward differences were used to 
calculate the gradient. This meant that the velocity had to be additionally calculated at the midpoint, and at three grid points in all three directions. The total number of the velocity estimations per time step would then be six. Because the most time consuming element of the calculation consisted of the velocity estimation, we decided to use the Taylor expansion in order to calculate the velocity at the drop positions as well (note the time reduction by the factor of 1.5.) Tests were performed to check whether the latter approximation would affect the trajectories. No effect was found on trajectories resulting in collision events. Consequently, neither was there any effect. on the value of the probability of collision.

\subsection{Numerical Procedures}

The system of equations (5.1)-(5.3) represents an initial value problem composed of 18 ordinary differential equations. The method of numerical solution of this system is based on Shampine \& Gordon [1975]. Also, the computer code is a modification of the one presented in this text. The predictor (Adams-Bashforth)-correctior (Adams-Moulton) method is used to approximate the solution. Let us schematically write the differential equation to be solved as

$$
y^{\prime}(x)=f(x, y(x))
$$

where prime denotes the derivative with respect to $x$. Both Adams schemes are based on the following integral equation to find the approximate vaiue of $y\left(x_{n+1}\right)\left(x_{n+1}\right.$ being a subsequent point of the mesh)

$$
y\left(x_{n+1}\right)=y\left(x_{n}\right)+\int_{x_{n}}^{x_{n+1}} f(t, y(t)) d t
$$

The function under integral is then approximated by a polynomial which uses the preceding points. Both Adams method use the Lagrange interpolation with backward 
differences. For the Adanis-Bashforth method of order $p$, points $x_{n-p}, \ldots, x_{n}$ serve as a basis ior the interpolation, while for the Adams-Moulton method of order $p$, points $x_{n-p+1}, \ldots, x_{n+1}$ serve the same purpose. Clearly, the former scheme is explicit while the latter implicit. However, the corrector scheme uses the value of $y\left(x_{n+1}\right)$ obtained in the prediction stage. Thus, practically, the predictor-corrector sequence works as an explicit scheme. The Adams family of predictor-corrector methods combine sufficient accuracy with very good stability properties. Shampine \& Gordon [1977] found that the scheme with the corrector one order lower than the predictor yields the best results. In the Shampine \& Gordon implementation of Adams methods, the mesh is variable, so is the order of the method. The criteria determining both the step size and the order of the method were based on the estimate of the local error associated with one step of iteration. This local error is calculated as a difference between the obtained value of the function and the value of the function which would be obtained if the order of the method was one order higher. The weighted local error must be smaller than the user supplied small number which represents the accuracy. (For the system of equations, the r.m.s. of the weighted local errors of all components was used instead.) A series of tests to determine the desired order of the accuracy was performed. For the trajectories which resulterin a collision, the accuracy was decreased to find out its upper limit. Based on the test results, a value of $10^{-7}$ was uniformly applied. The choice of the initial time step turned out to be quite insignificant-the method given a reasonable first guess handled it by itself. 


\subsection{Trajectory Simulations}

The method, as described in the preceding sections, was subsequently used to model trajectories. Here, we present the examples of trajectories of both types: laminar and turbulent. A few comments are in order. Firstly, the SRF's were interpolated for turbulent calculations, while for laminar calculations the coefficients were calculated directly. Secondly, the system of equations for the laminar case reduces to only five equations because the flow becomes two-dimensional: two components of both position and velocity ( $x$ and $z$ ), and one component of angular velocity perpendicular to the plane of motion $(y)$. In our convention the motion takes place on the $x-z$ plane.

In order to illustrate the interaction between drops of different, sizes, we have selected two drop pairs: 15 and $8 \mu \mathrm{m}$, and 15 and $14 \mu \mathrm{m}$. The laminar trajectories to be presented are chosen in such a way that the drops do not collide but pass by each other closely. In Figs. 5.4a, b and 5.5a, b (laminar cases), the solutions of the differential equations are plotted vs. time. Note that only the final time period is captured in the figures-the initial period of time with weak interactions amounts to straight-line motion, and, therefore, is not presented. The initial vertical separation is equal to that used for grazing trajectory calculations, $z^{2}-z^{1}=0.7 \mathrm{~cm}$. The horizontal offset is slightly larger than that corresponding to the grazing trajectory, $x^{2}-x^{1}=28$ $\mu \mathrm{m}$ for 15 and $8 \mu \mathrm{m}$ drops, and $x^{2}-x^{1}=32 \mu \mathrm{m}$ for 15 and $14 \mu \mathrm{m}$ drops. The initial velocities of drops are equal to their terminal velocities. The initial angular velocities are equal to zero. On each of Figs. 5.4a and 5.5a, there are six panels. Left-hand-side panels feature (from top to bottom) the drop positions, $x$ and $z$, and the distance between drops $D$. The latter is plotted in order to better visualize the time period when drops are in a close proximity. The right-hand-side panels contain the velocity 
components, $v_{x}$ and $v_{z}$, and the angular velocity, $\Omega_{y}$. Additionally, in Figs. $5.4 \mathrm{~b}$ and $5.5 \mathrm{~b}$, the relative coordinates $\left(x^{1}-x^{2}\right.$ and $\left.z^{1}-z^{2}\right)$ and the relative velocities $\left(v_{x}^{1}-v_{x}^{2}\right.$ and $v_{z}^{1}-v_{z}^{2}$ ) are plotted vs. time to facilitate comparisons. The major difference between the behaviour of pairs of drops witli small and large radius ratios can be synthesized to only two elements: distortion of trajectories and time of interaction. For the 15 and $8 \mu \mathrm{m}$ drop pair, the smaller drop experience a much more pronounced disturbance in the $x$-direction than the larger one, while for drops 15 and $14 \mu \mathrm{m}$, the character of the disturbances is similar for both droplets. The time of interaction is also different. The comparison of the distances between droplets for both pairs shows clearly that drops of 15 and $14 \mu \mathrm{m}$ are in a close proximity (the flat part of the curve) for longer time than drops of 15 and $8 \mu \mathrm{m}$ : approximately 10 times longer. We will return to these two aspects of the interaction between droplets while discussing the results for collision rates.

Turbulent calculations are presented in Figs. $5.6 \mathrm{a}, \mathrm{b}, \mathrm{c}$ and $5.7 \mathrm{a}, \mathrm{b}, \mathrm{c}(\epsilon=1$ $\left.\mathrm{cm}^{2} \mathrm{sec}^{-3}\right)$, Figs. 5.8a, b, c and 5.9a, b, c $\left(\epsilon=10 \mathrm{~cm}^{2} \mathrm{sec}^{-3}\right)$, and Figs. $5.10 \mathrm{a}, \mathrm{b}$, c and $5.11 \mathrm{a}, \mathrm{b}, \mathrm{c}\left(\epsilon=100 \mathrm{~cm}^{2} \mathrm{sec}^{-3}\right)$. Each case results in a collision. The initial distance between drops $R=\left|\mathbf{x}^{2}-\mathbf{x}^{1}\right|=0.03 \mathrm{~cm}$ (except for drops 15 and $14 \mu \mathrm{m}$, and $\epsilon=100 \mathrm{~cm}^{2} \mathrm{sec}^{-3}$, where $R=0.015 \mathrm{~cm}$.) These are the distances used later in the calculation of collision kernels. In order to describe the drop initial positions for turbulent calculations, we use the spherical coordinate system $(r, \theta, \phi)$ with the collector drop placed in the origin (the reasons will be explained in Chapter 6.) Therefore, the distance $R$ and the angle $\theta$ of the smaller drop are the two parameters determining the initial, relative drop positions. (The angle $\phi$ of the smaller drop is always assumed to be zero.) The chosen examples represent trajectories with the angles $\theta$ significantly wider than those for the corresponding grazing trajectories (see figure captions). The initial drop velocities are equal to the superposition of 
their terminal velocities and the turbulent velocities at their initial coordinates. The initial angular velocities are equal to zero. The drop coordinates are plotted on the left-hand-side panels of the ' $a$ ' figures, the drop velocity components on the righthand-side panels of the 'a' figures, and the drop angular velocities on the left-handside panels of the ' $b$ ' figures. The right--hand-side panels of the ' $b$ ' figures arc reserved for the ambient turbulent velocity field at drop coordinates. The latter are included in order to illustrate the hydrodynamic interactions. Note that in the horizontal planc, if not disturbed by their mutual presence, drops follow closely the trajectories of fluid parcel. Finally, the 'c' figures show the differences of the coordinates and the velocity components of two drops.

No conclusions can be derived from turbulent trajectories in the same manner as it was done for laminar ones. This is because for turbulent calculation orly the quantities which are appropriately averaged can shed some light on the occurring processes. Figs. 5.6-5.11, however, clearly show that as the ratio of drop radii becomes larger and the rate of energy dissipation stronger, the character of the curves resembles less and less that of the laminar solution. From the 'technical' point of view, note that the initial parts of some of the solutions show the relaxation of the initial conditions. 

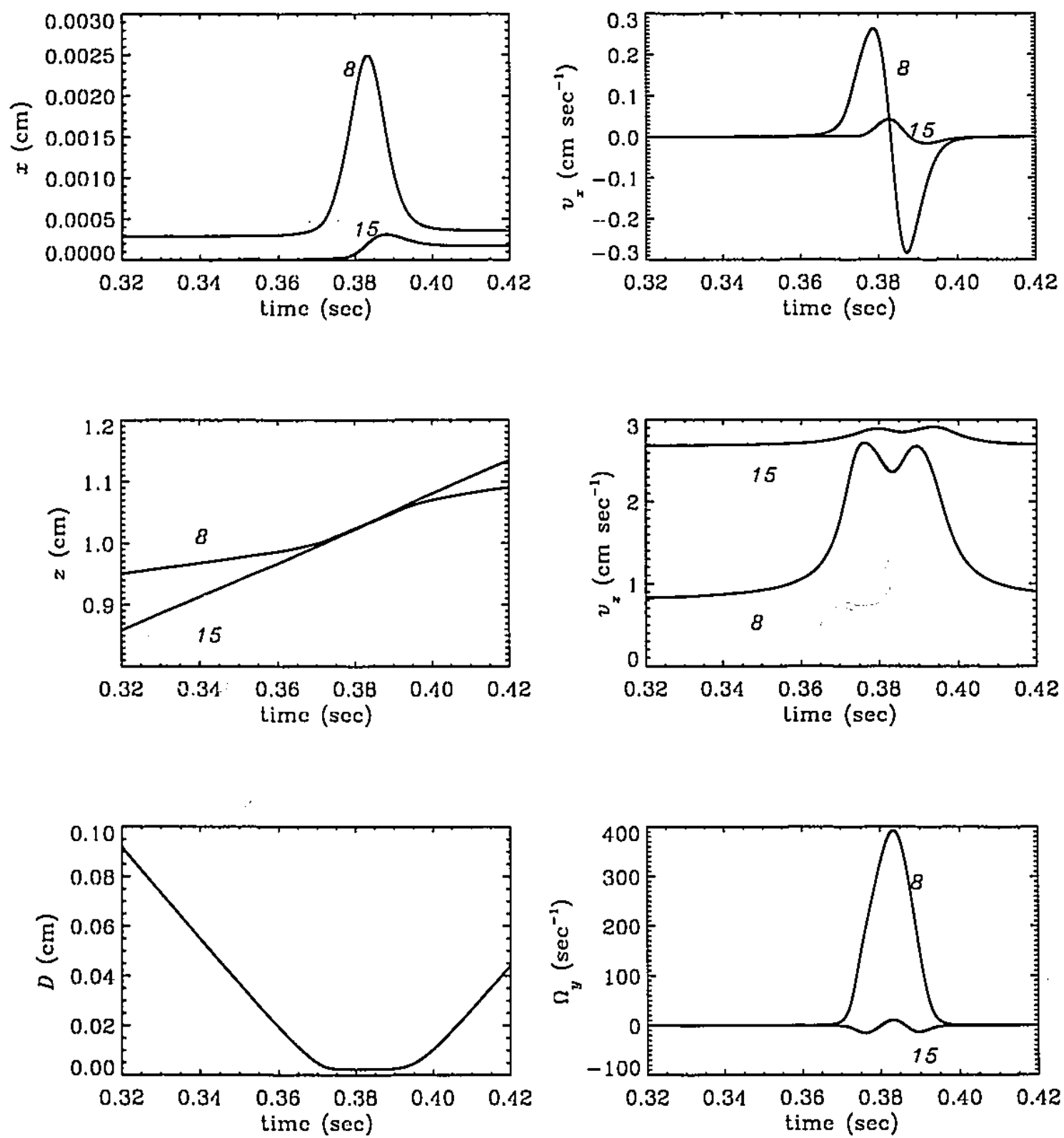

Fig. 5.4a The drop coordinates, $x$ and $z$, the relative distance between drops, $D$, the drop velocity components, $v_{x}$ and $v_{z}$, and the drop angular velocity component, $\Omega_{y}$ vs. time for the laminar settling of 15 and $8 \mu \mathrm{m}$ drops. Numbers indicate radii. No collision occurs. 

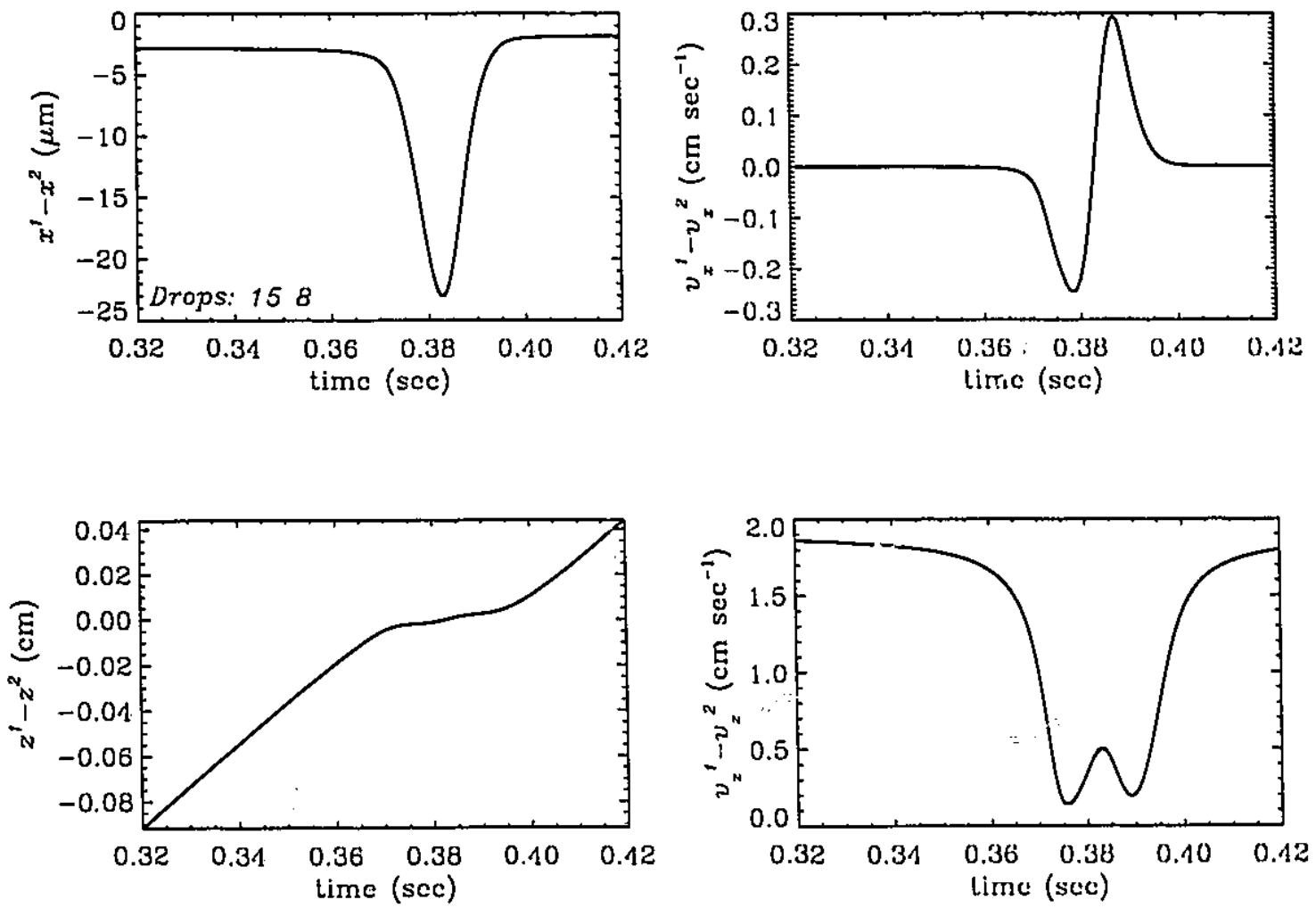

Fig. 5.4b The drop relative coordinates, $x^{1}-x^{2}$ and $z^{1}-z^{2}$, and the drop relative velocity components, $v_{x}^{1}-v_{x}^{2}$ and $v_{z}^{1}-v_{z}^{2}$ vs. time for the laminar settling of 15 and $8 \mu \mathrm{m}$ drops. No collision occurs. 

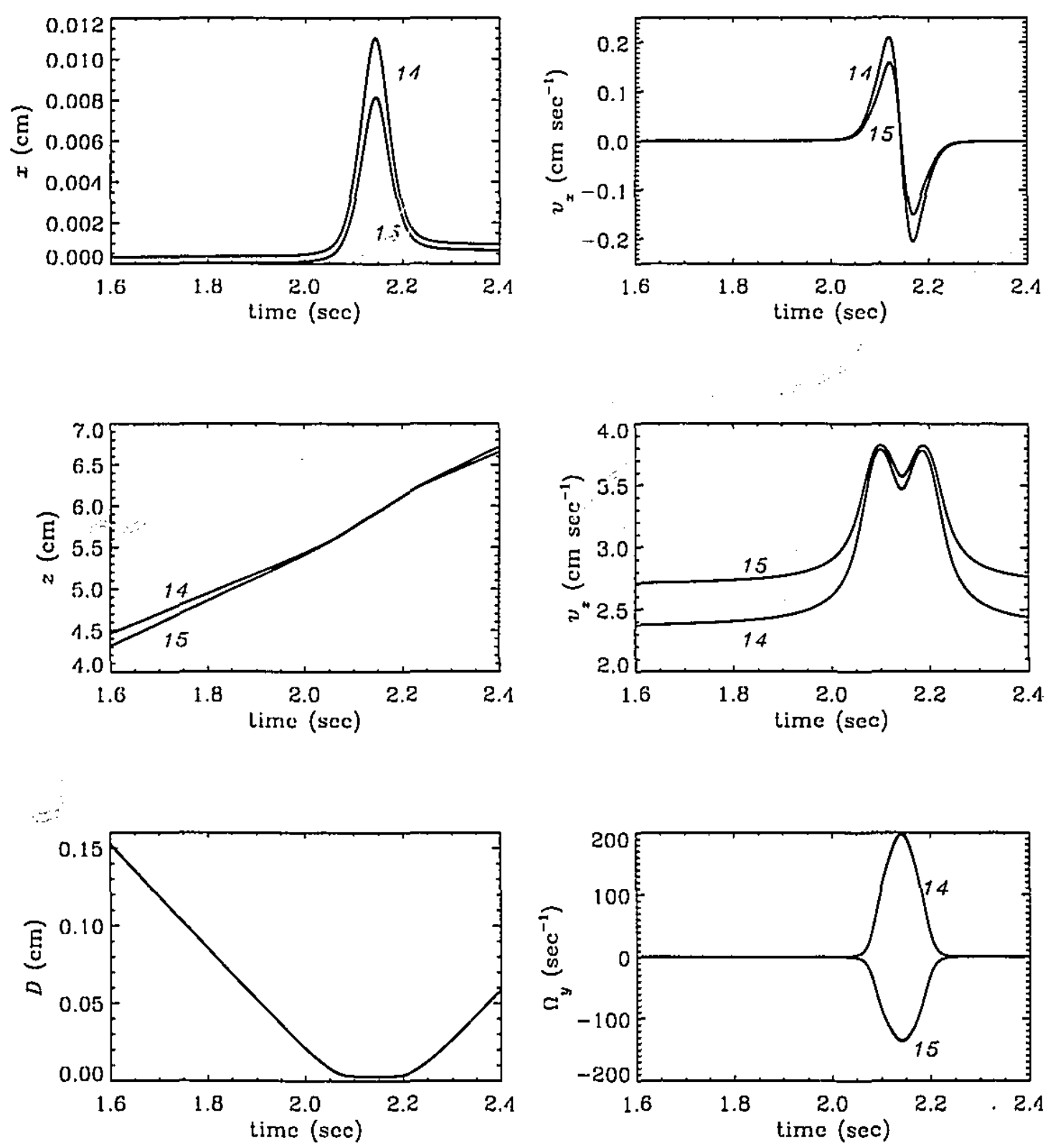

Fig. 5.5a The drop ccordinates, $x$ and $z$, the relative distance between drops, $D$, the drop velocity components, $v_{x}$ and $v_{z}$, and the drop angular velocity component, $\Omega_{y}$ vs. time for the laminar settling of 15 and $14 \mu \mathrm{m}$ drops. Numbers indicate radii. No collision occurs. 

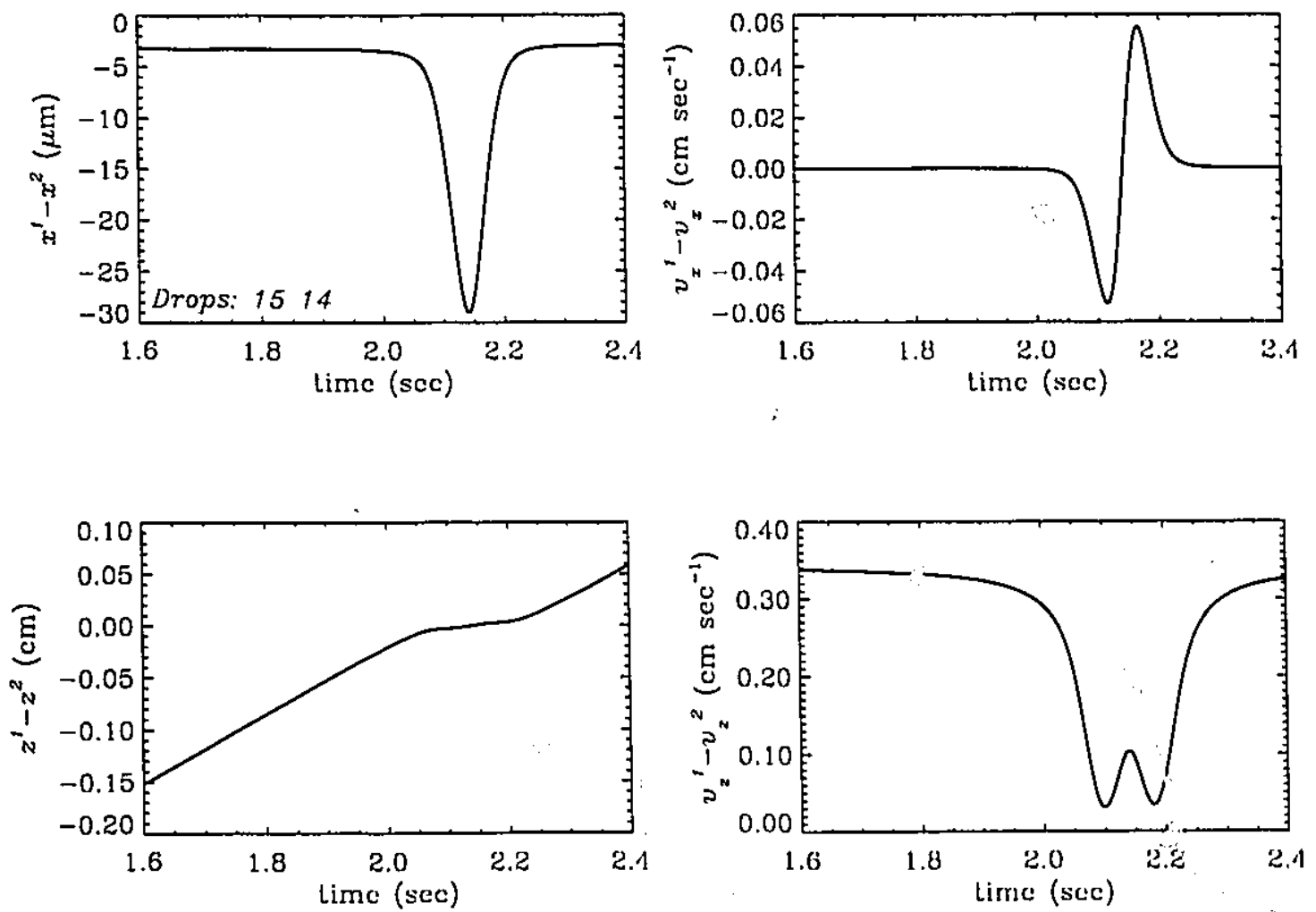

Fig. 5.5b The drop relative coordinates, $x^{1}-x^{2}$ and $z^{1}-z^{2}$, and the drop relative velocity components, $v_{x}^{1}-v_{x}^{2}$ and $v_{z}^{1}-v_{z}^{2}$ vs. time for the laminar settling of 15 and $14 \mu \mathrm{m}$ drops. No collision occurs. 

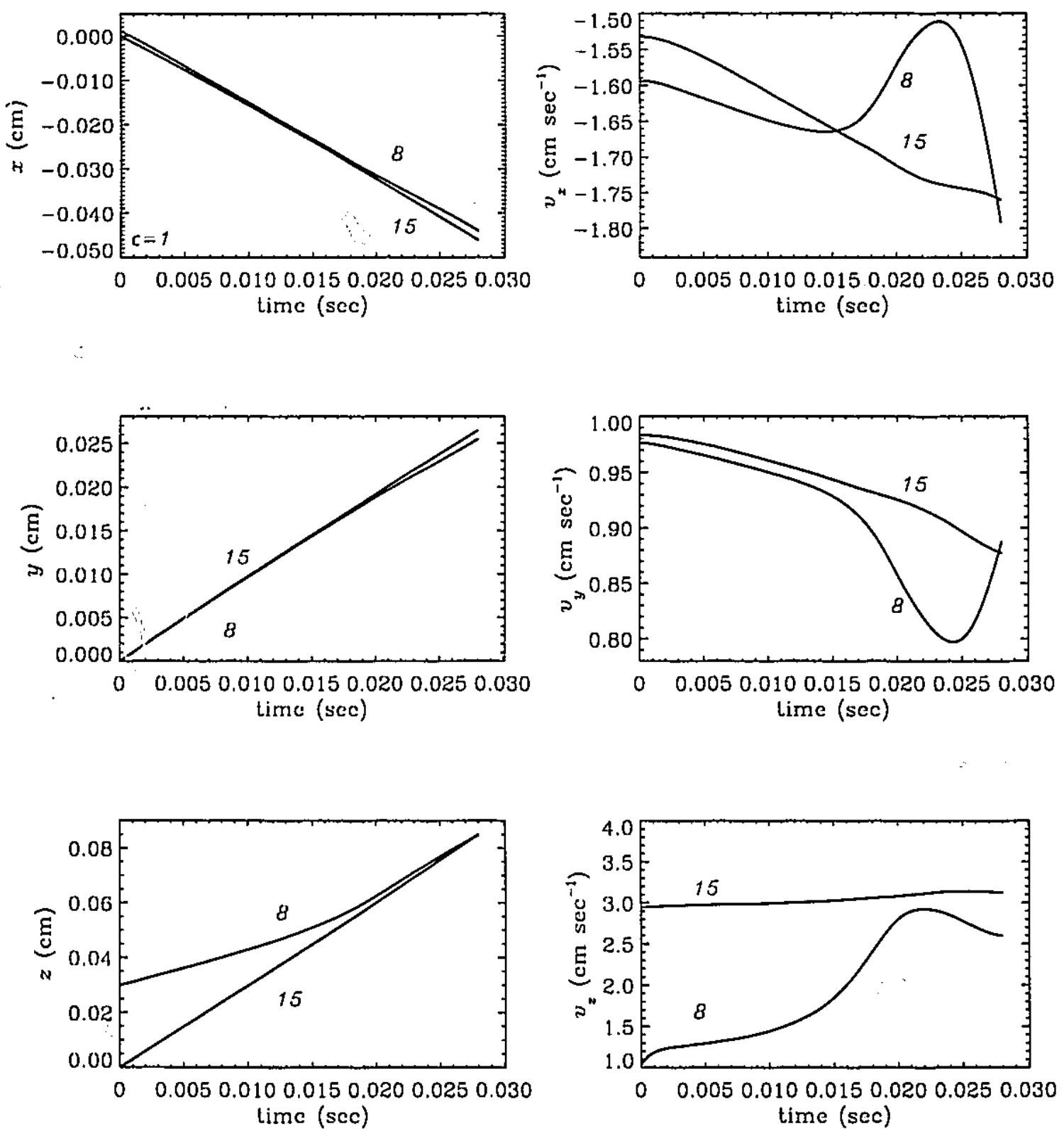

Fig. 5.6a The drop coordinates, $x, y$, and $z$, and the drop velocity components, $v_{x}$, $v_{y}$, and $v_{z}$ vs. time for the turbulent settling of 15 and $8 \mu \mathrm{m}$ drops and the rate of energy dissipation $1 \mathrm{~cm}^{2} \mathrm{sec}^{-3}$. The initial position of the smaller drop: $R=0.03 \mathrm{~cm}$ and $\theta=2.25^{\circ}$. Numbers indicate radii. The calculation results in a collision. 

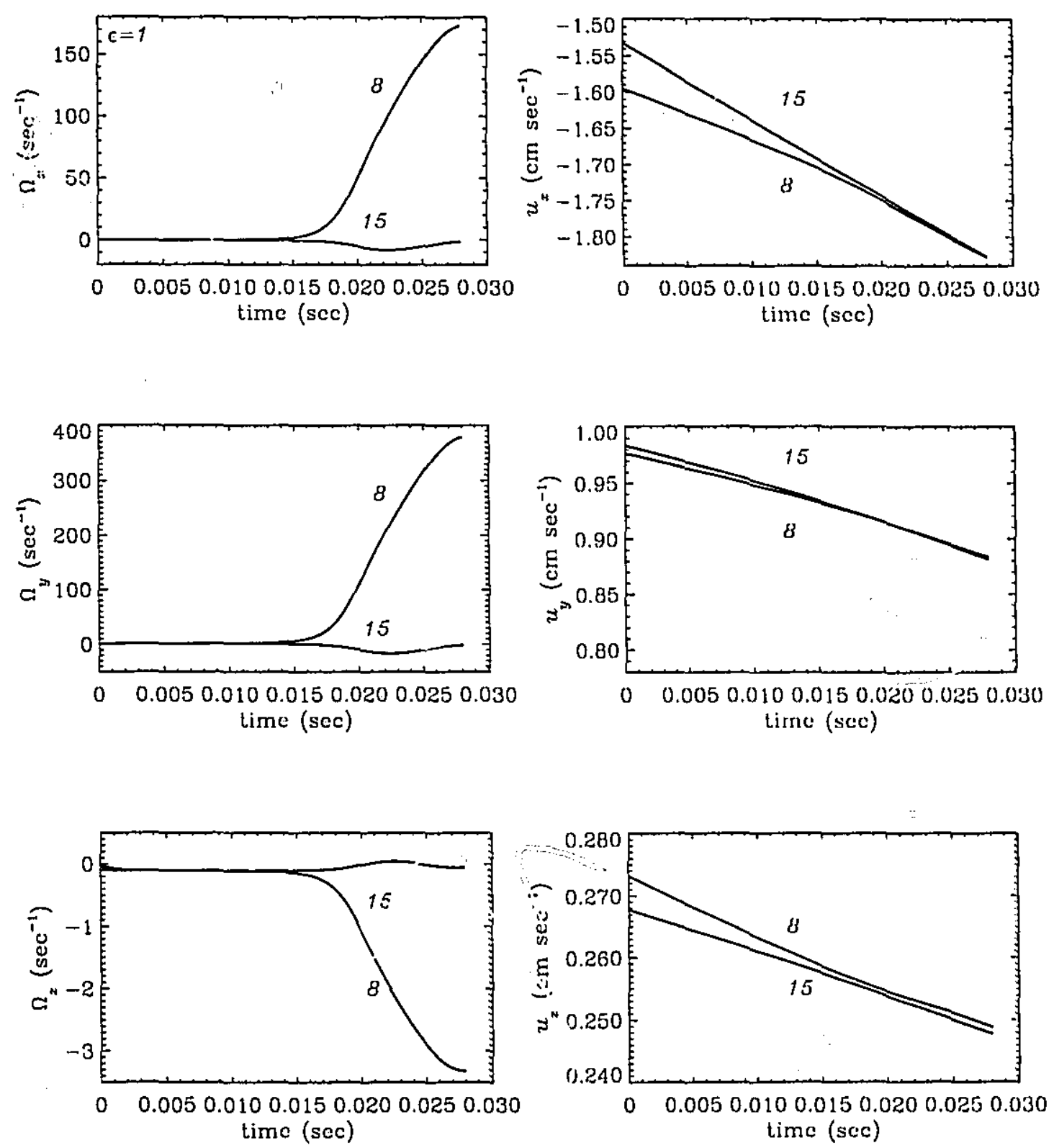

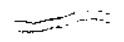

Fig. 5.6b The drop angular velocity components, $\Omega_{x}, \Omega_{y}$, and $\Omega_{z}$, and the turbulent. velocity components, $u_{x}, u_{y}$, and $u_{z}$ at drop coordinates vs. time for the turbulent settling of 15 and $8 \mu \mathrm{m}$ drops and the rate of energy dissipation $1 \mathrm{~cm}^{2}$ $\mathrm{sec}^{-3}$. The initial position of the smaller drop: $R=0.03 \mathrm{~cm}$ and $\theta=2.25^{\circ}$. Numbers indicate radii. The calculation results in a collision. 

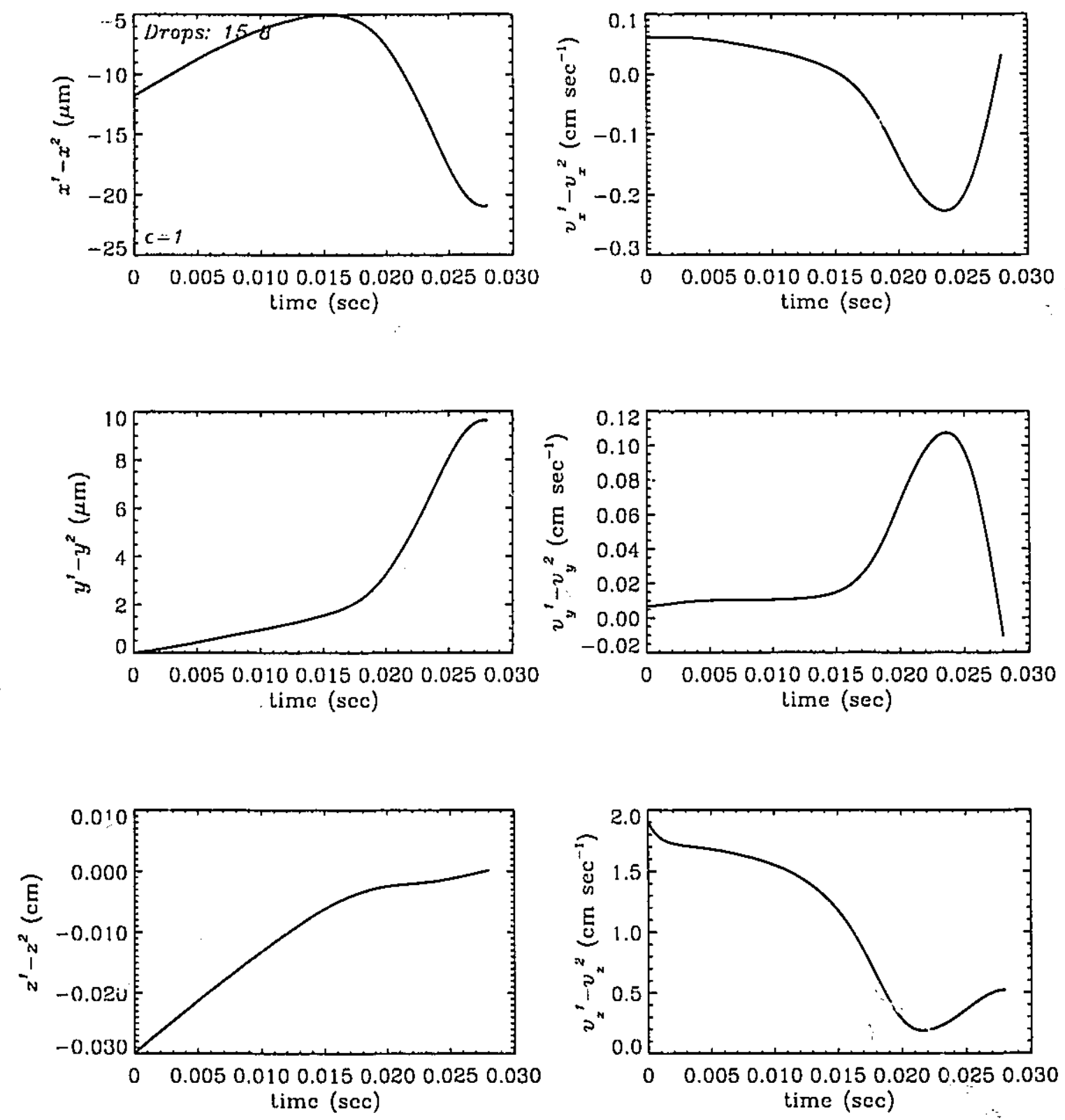

Fig. 5.6c The drop relative coordinates, $x^{1}-x^{2}, y^{1}-y^{2}$, and $z^{1}-z^{2}$, and the drop relative velocity components, $v_{x}^{1}-v_{x}^{2}, v_{y}^{1}-v_{y}^{2}$, and $v_{z}^{1}-v_{z}^{2}$ vs. time for the turbulent settling of 15 and $8 \mu \mathrm{m}$ drops and the rate of energy dissipation 1 $\mathrm{cm}^{2} \mathrm{sec}^{-3}$. The initial position of the smaller drop: $R=0.03 \mathrm{~cm}$ and $\theta=2.25^{\circ}$. The calculation results in a collision. 

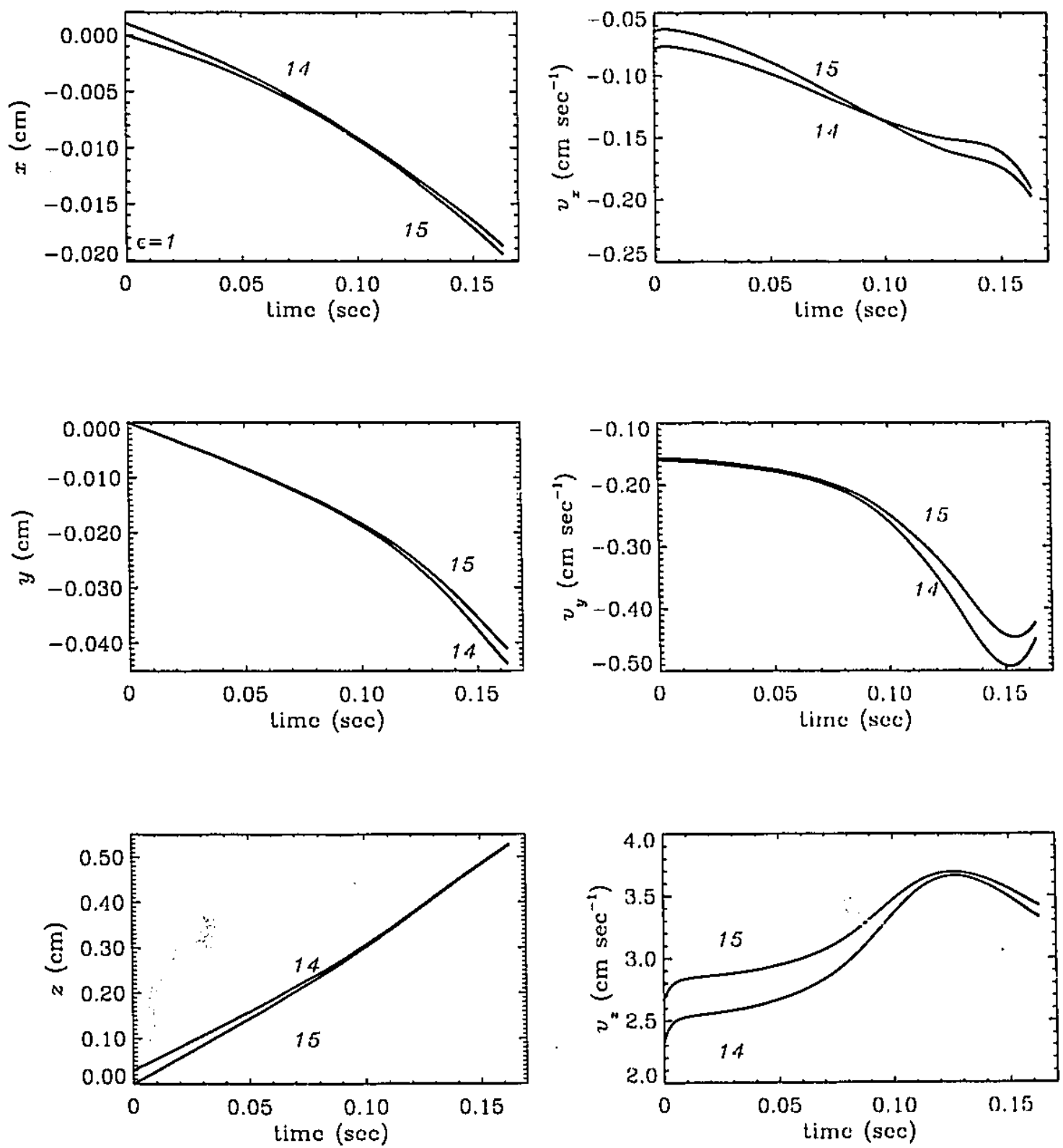

Fig. 5.7a The drop coordinates, $x, y$, and $z$, and the drop velocity components, $v_{x}$, $v_{y}$, and $v_{z}$ vs. time for the turbulent settling of 15 and $14 \mu \mathrm{m}$ drops and the rate of energy dissipation $1 \mathrm{~cm}^{2} \mathrm{sec}^{-3}$. The initial position of the smaller clrop: $R=0.03 \mathrm{~cm}$ and $\theta=2^{\circ}$. Numbers indicate radii. The calculation results in a collision. 

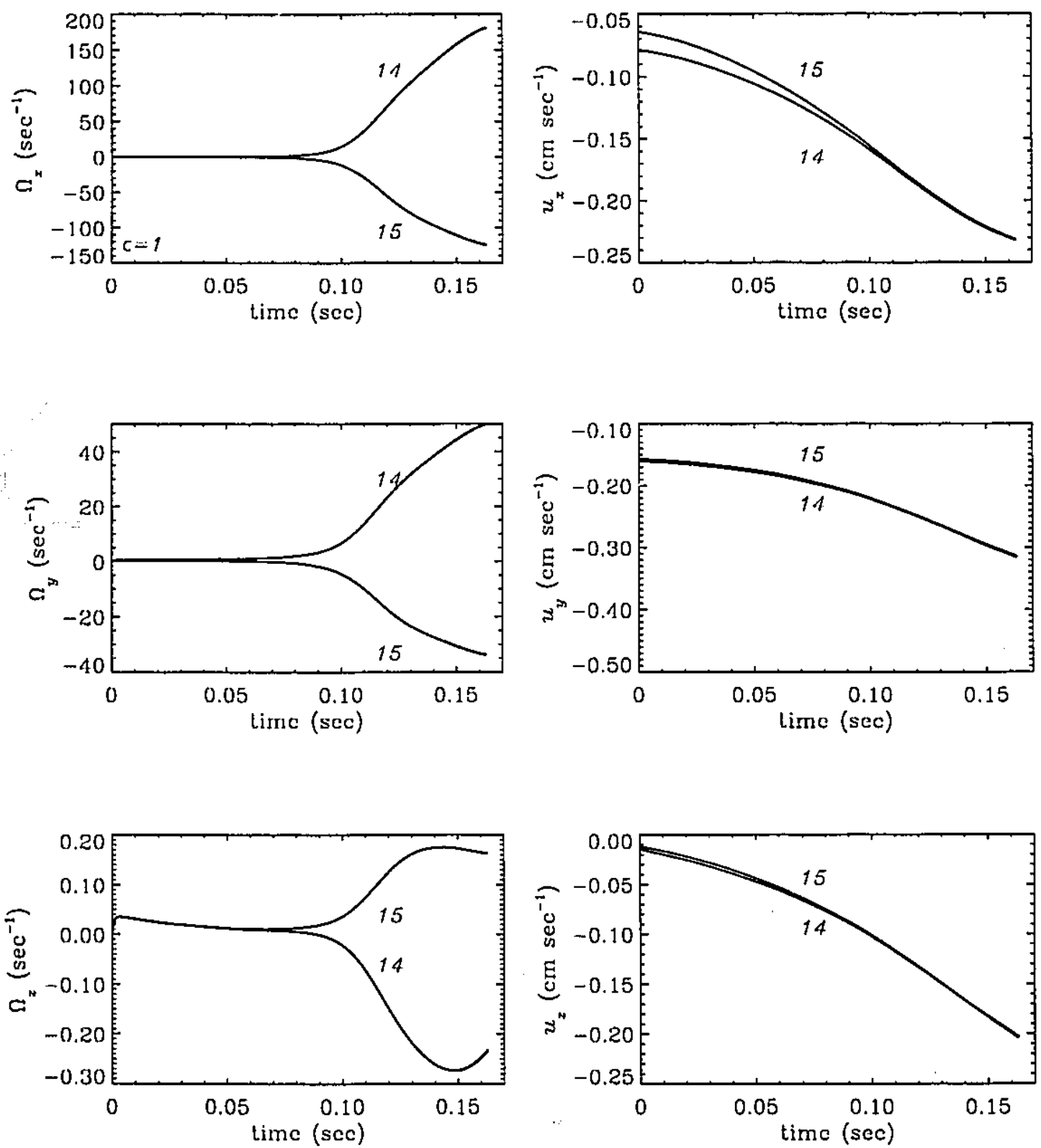

Fig. 5.7b The drop angular velocity components, $\Omega_{x}, \Omega_{y}$, and $\Omega_{z}$, and the turbulent velocity components, $u_{x}, u_{y}$, and $u_{z}$ at drop coordinates vs. time for the turbulent settling of 15 and $14 \mu \mathrm{m}$ drops and the rate of energy dissipation 1 $\mathrm{cm}^{2} \mathrm{sec}^{-3}$. The initial position of the smaller drop: $R=0.03 \mathrm{~cm}$ and $\theta=2^{\circ}$. Numbers indicate radii. The calculation results in a collision. 

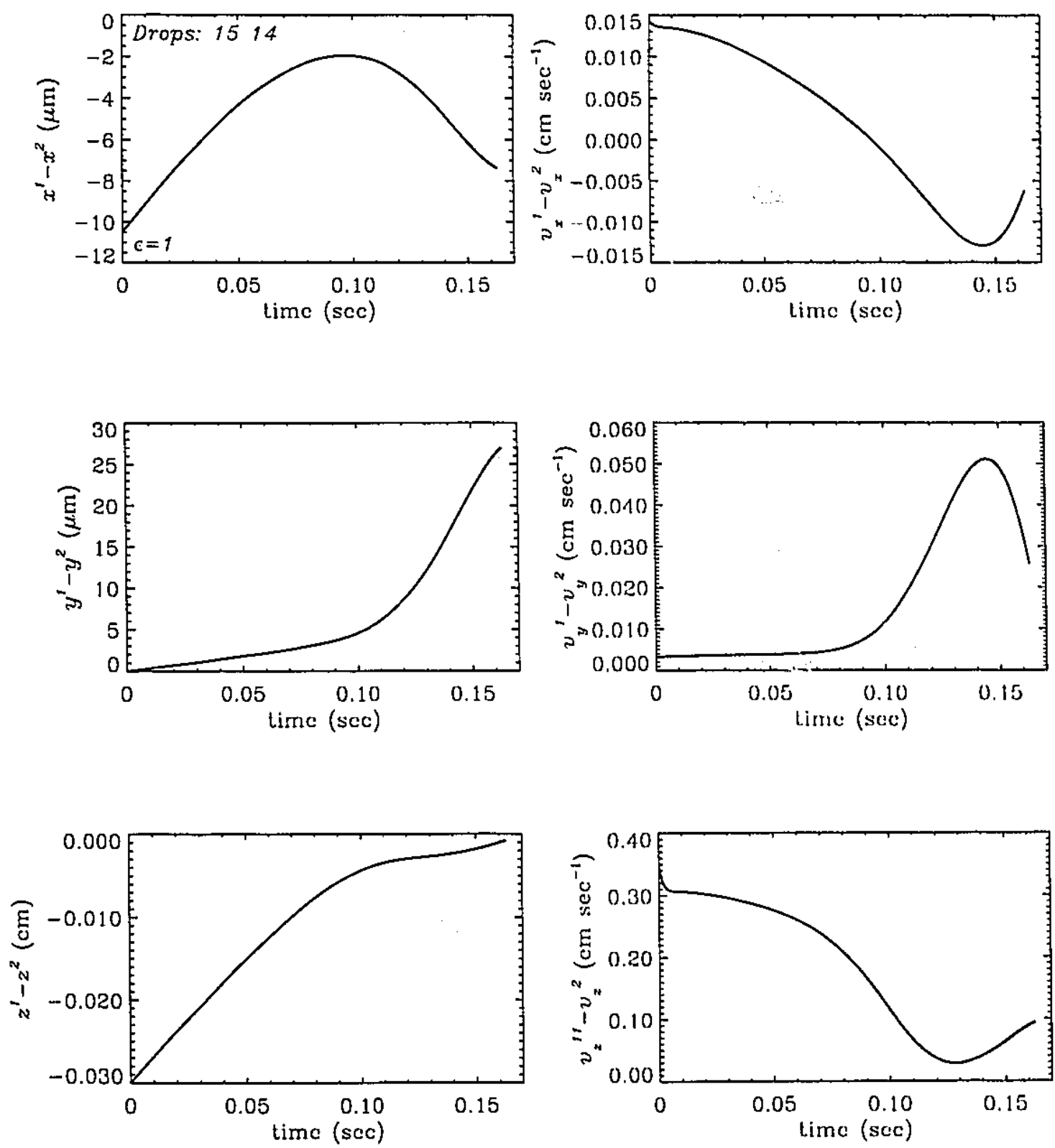

Fig. 5.7c The drop relative coordinates, $x^{1}-x^{2}, y^{1}-y^{2}$, and $z^{1}-z^{2}$, and the drop relative velocity components, $v_{x}^{1}-v_{x}^{2}, v_{y}^{1}-v_{y}^{2}$, and $v_{z}^{1}-v_{z}^{2}$ vs. time for the turbulent settling of 15 and $14 \mu \mathrm{m}$ drops and the rate of energy dissipation 1 $\mathrm{cm}^{2} \mathrm{sec}^{-3}$. The initial position of the smaller drop: $R=0.03 \mathrm{~cm}$ and $\theta=2^{\circ}$. The calculation results in a collision. 

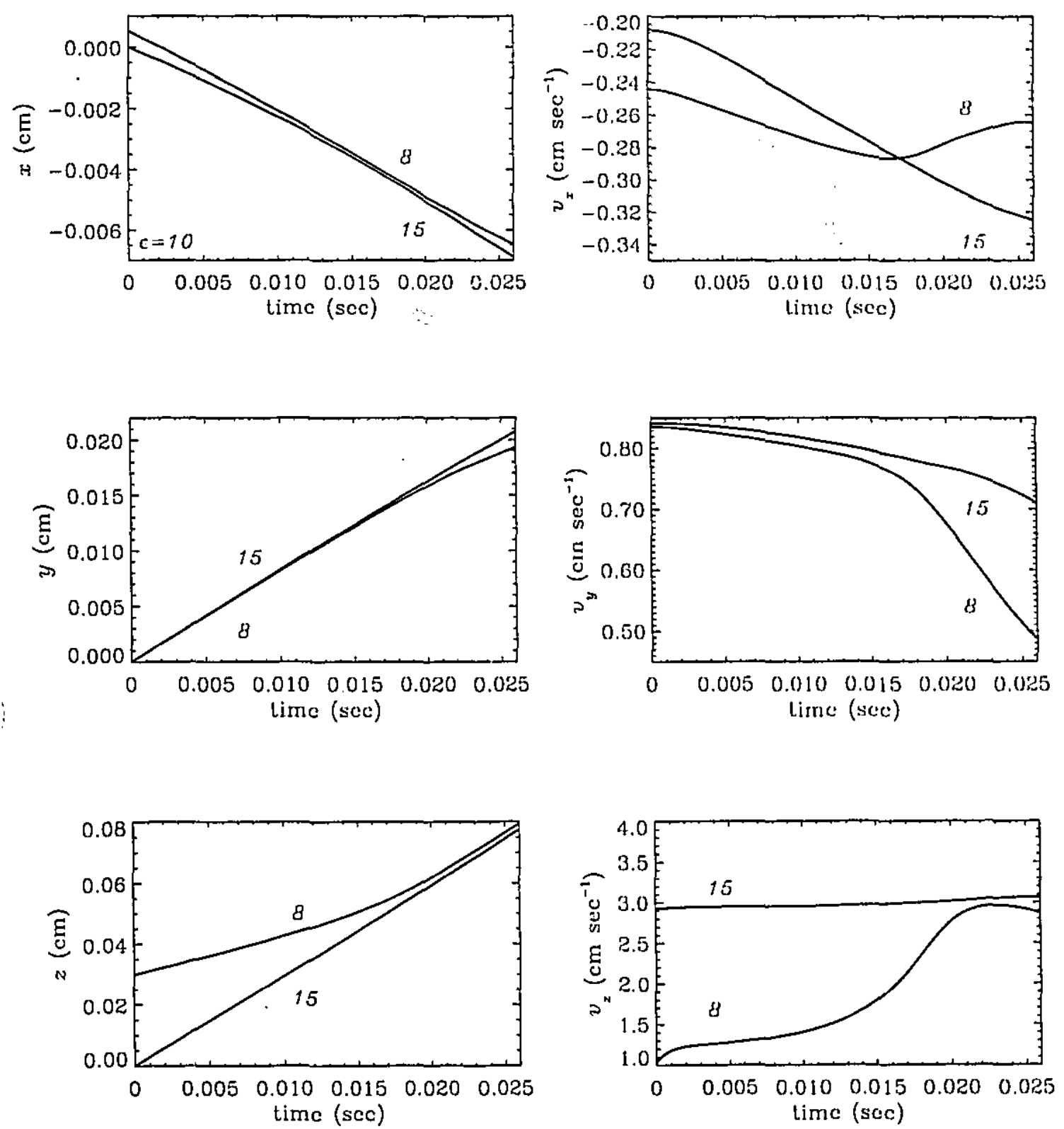

Fig. 5.8a The drop coordinates, $x, y$, and $z$, and the drop velocity components, $v_{x}$, $v_{y}$, and $v_{z}$ vs. time for the turbulent settling of 15 and $8 \mu \mathrm{m}$ drops and thic rate of energy dissipation $10 \mathrm{~cm}^{2} \mathrm{sec}^{-3}$. The initial position of the smaller drop: $R=0.03 \mathrm{~cm}$ and $\theta=1^{\circ}$. Numbers indicate radii. The calculation results in a collision. 

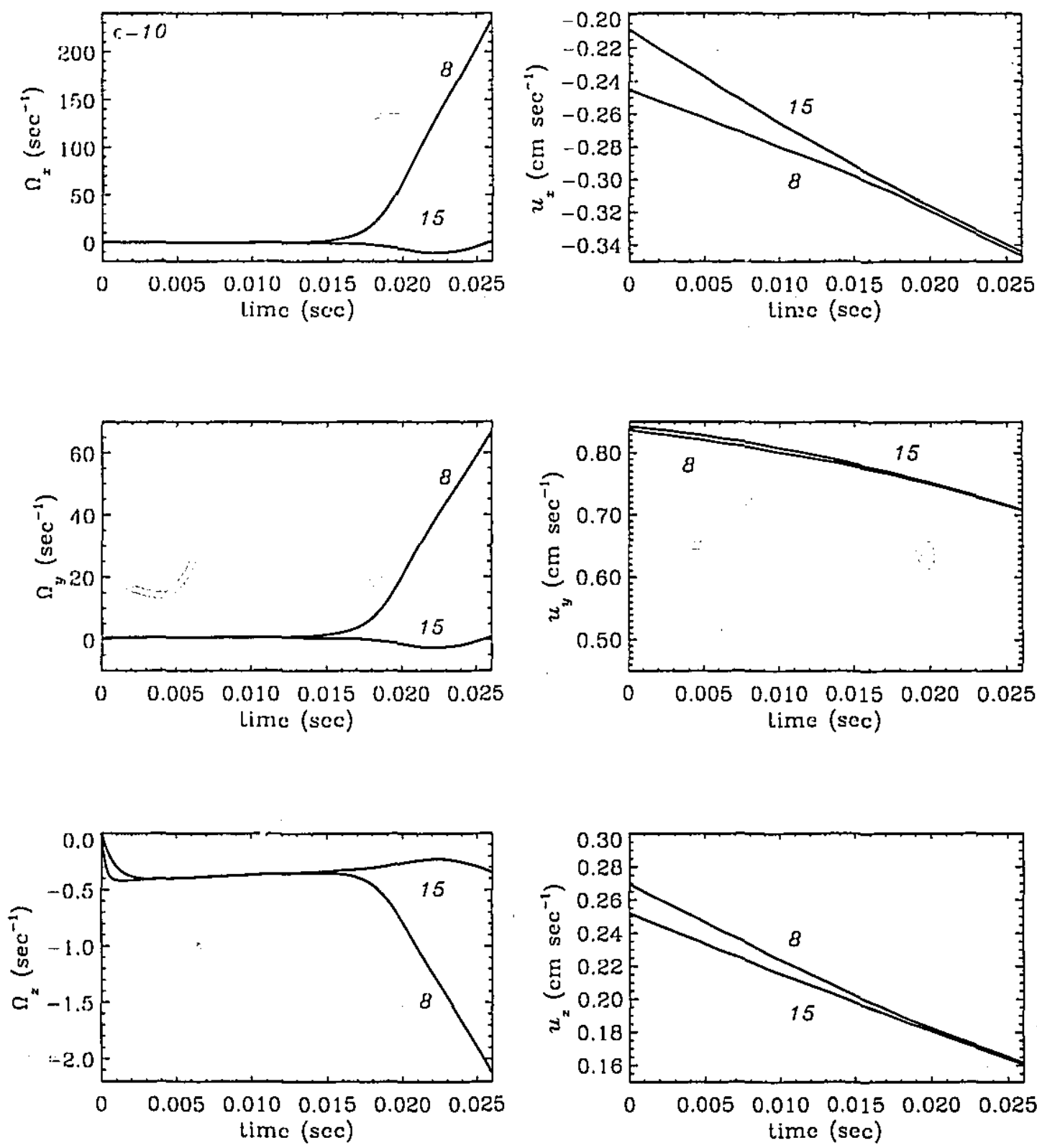

Fig. 5.8b The drop angular velocity components, $\Omega_{x}, \Omega_{y}$, and $\Omega_{z}$, and the turbulent velocity components, $u_{x}, u_{y}$, and $u_{z}$ at drop coordinates vs. time for the turbulent settling of 15 and $8 \mu \mathrm{m}$ drops and the rate of energy dissipation 10 $\mathrm{cm}^{2} \mathrm{sec}^{-3}$. The initial position of the smaller drop: $R=0.03 \mathrm{~cm}$ and $\theta=1^{\circ}$. Numbers indicate radii. The calculation results in a collision. 

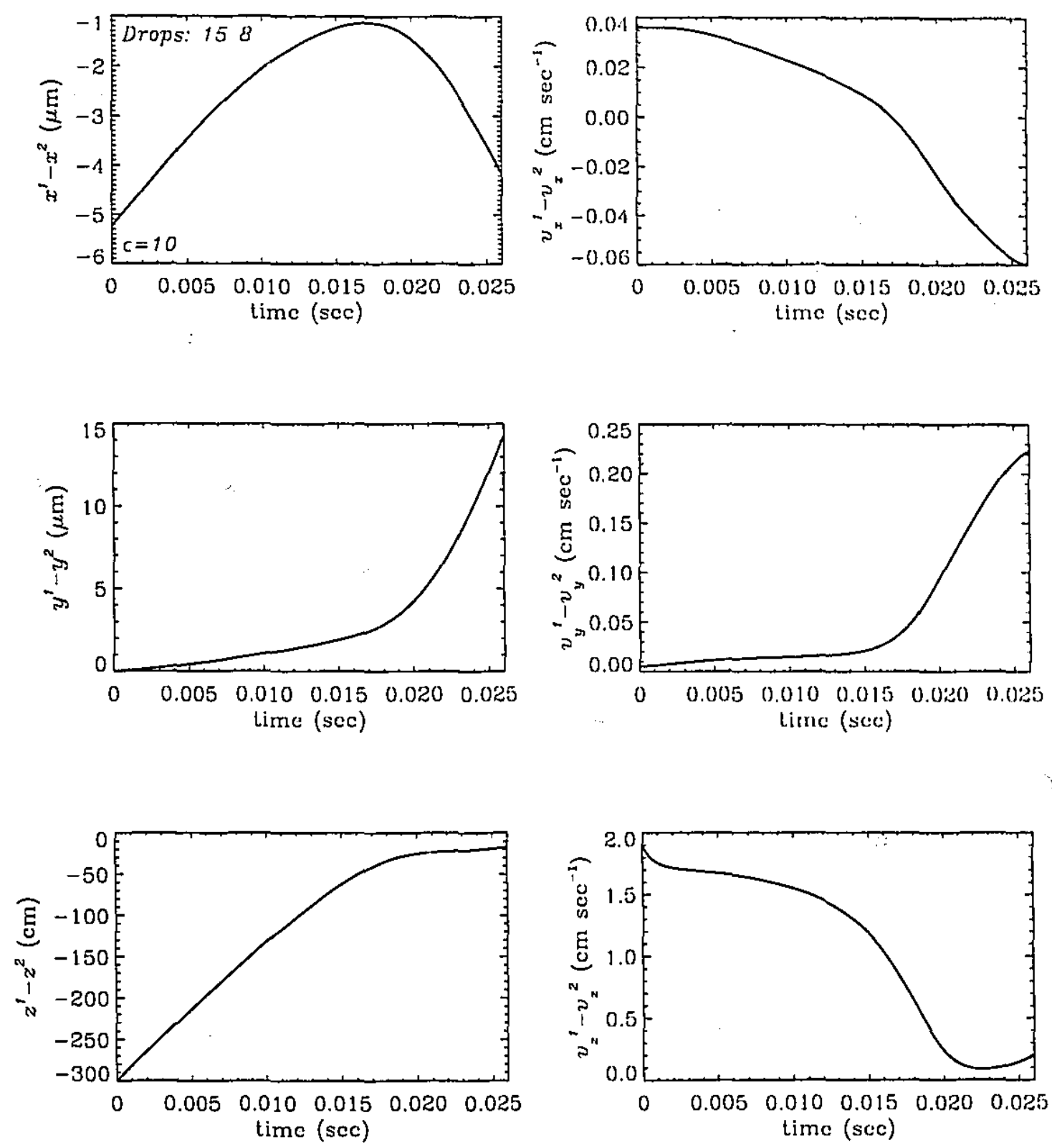

Fig. 5.8c The drop relative coordinates, $x^{1}-x^{2}, y^{1}-y^{2}$, and $z^{1}-z^{2}$, and the drop relative velocity components, $v_{x}^{1}-v_{x}^{2}, v_{y}^{1}-v_{y}^{2}$, and $v_{z}^{1}-v_{z}^{2}$ vs. time for the turbulent settling of 15 and $8 \mu \mathrm{m}$ drops and the rate of energy dissipation 10 $\mathrm{cm}^{2} \mathrm{sec}^{-3}$. The initial position of the smaller drop: $R=0.03 \mathrm{~cm}$ and $\theta=1^{\circ}$. The calculation results in a collision. 

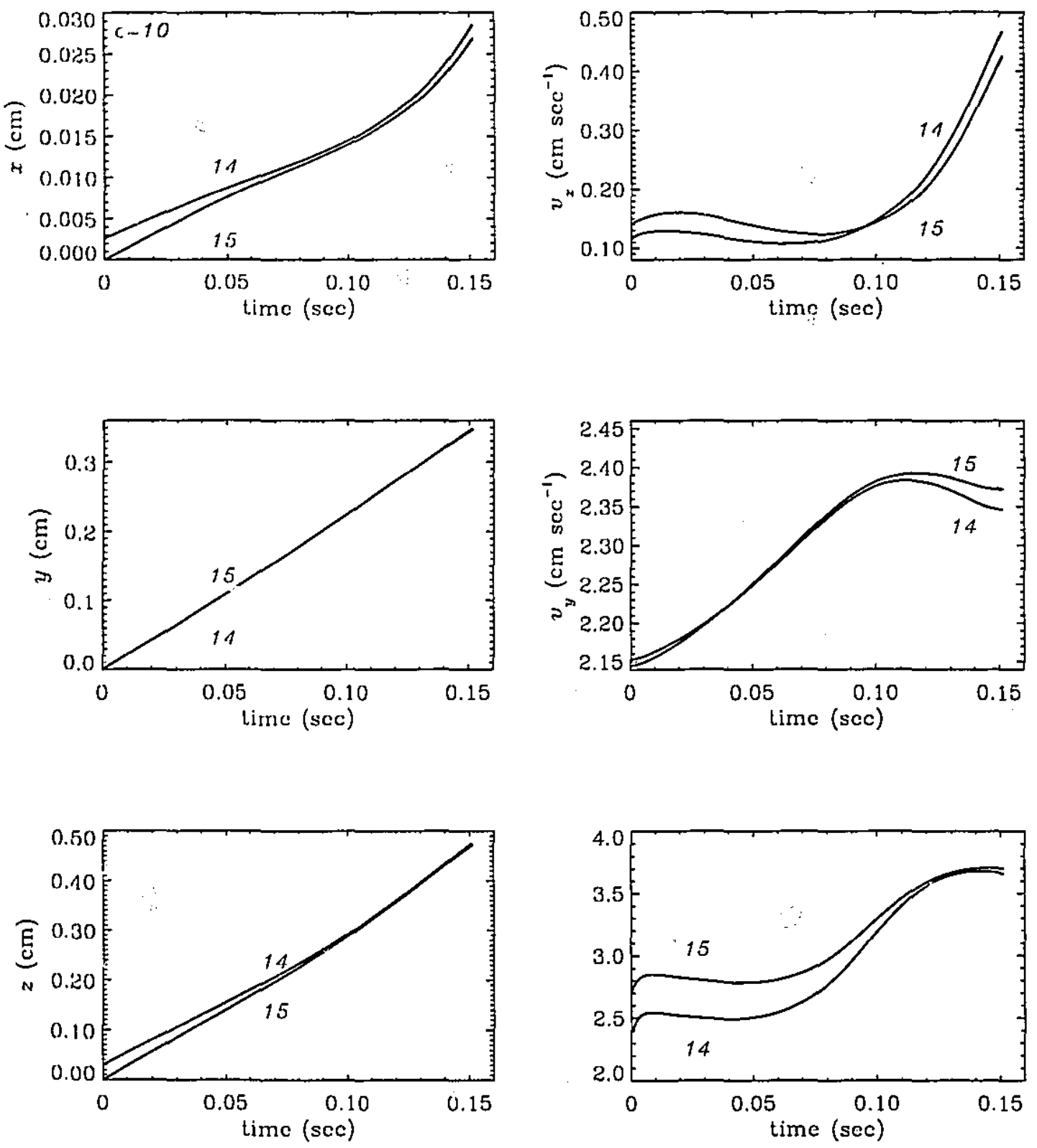

Fig. 5.9a The drop coordinates, $x, y$, and $z$, and the drop velocity components, $v_{x}$, $v_{y}$, and $v_{z}$ vs. time for the turbulent settling of 15 and $14 \mu \mathrm{m}$ drops and the rate of energy dissipation $10 \mathrm{~cm}^{2} \mathrm{sec}^{-3}$. The initial position of the smaller drop: $R=0.03 \mathrm{~cm}$ and $\theta=5^{\circ}$. Numbers indicate radii. The calculation results in a collision. 

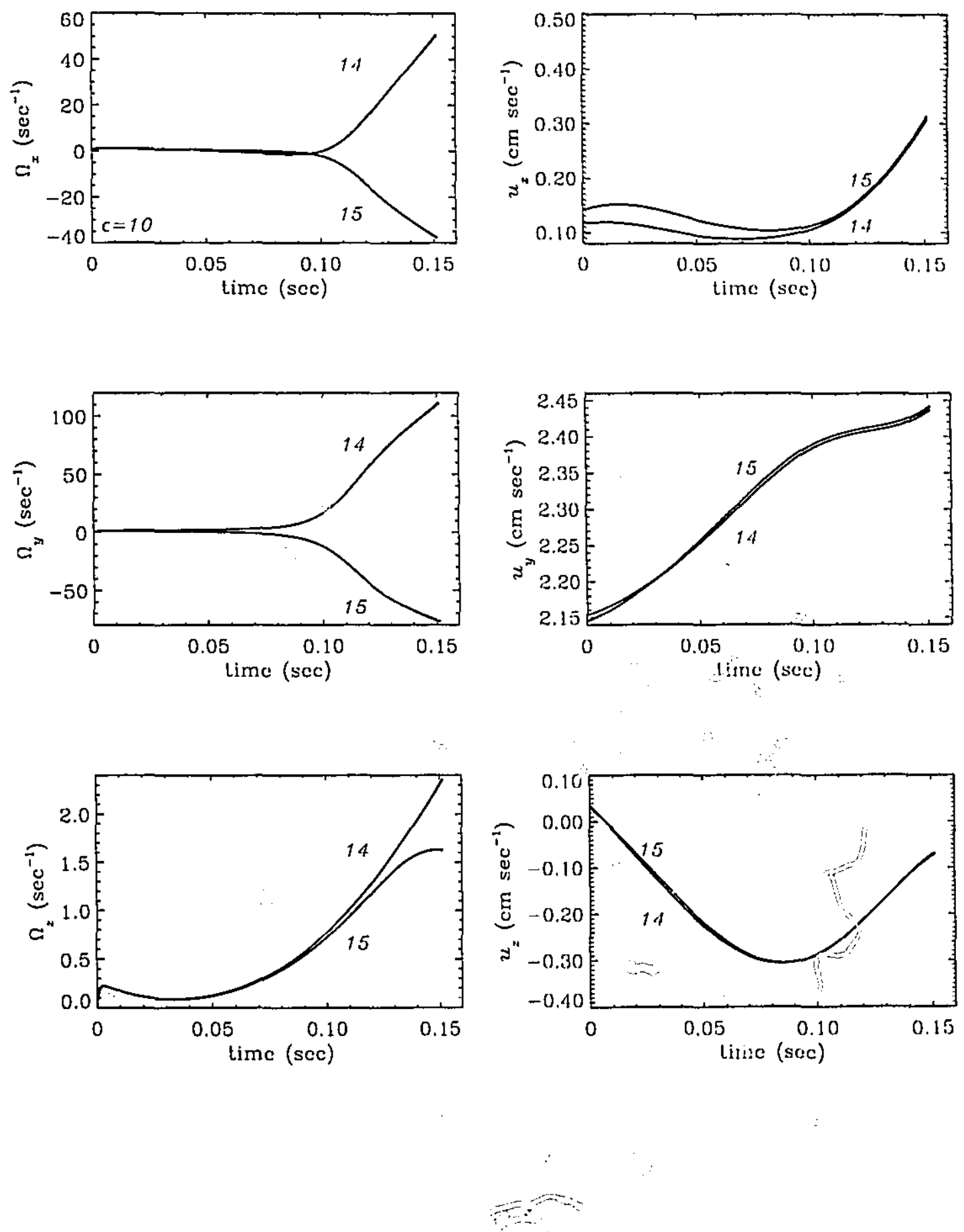

Fig. 5.9b The drop angular velocity components, $\Omega_{x}, \Omega_{y}$, and $\Omega_{z}$, and the turbulent velocity components, $u_{x}, u_{y}$, and $u_{z}$ at drop coordinates vs. time for the turbulent settling of 15 and $14 \mu \mathrm{m}$ drops and the rate of energy dissipation 10 $\mathrm{cm}^{2} \mathrm{sec}^{-3}$. The initial position of the smaller drop: $R=0.03 \mathrm{~cm}$ and $0=5^{\circ}$. Numbers indicate radii. The calculation results in a collision. 

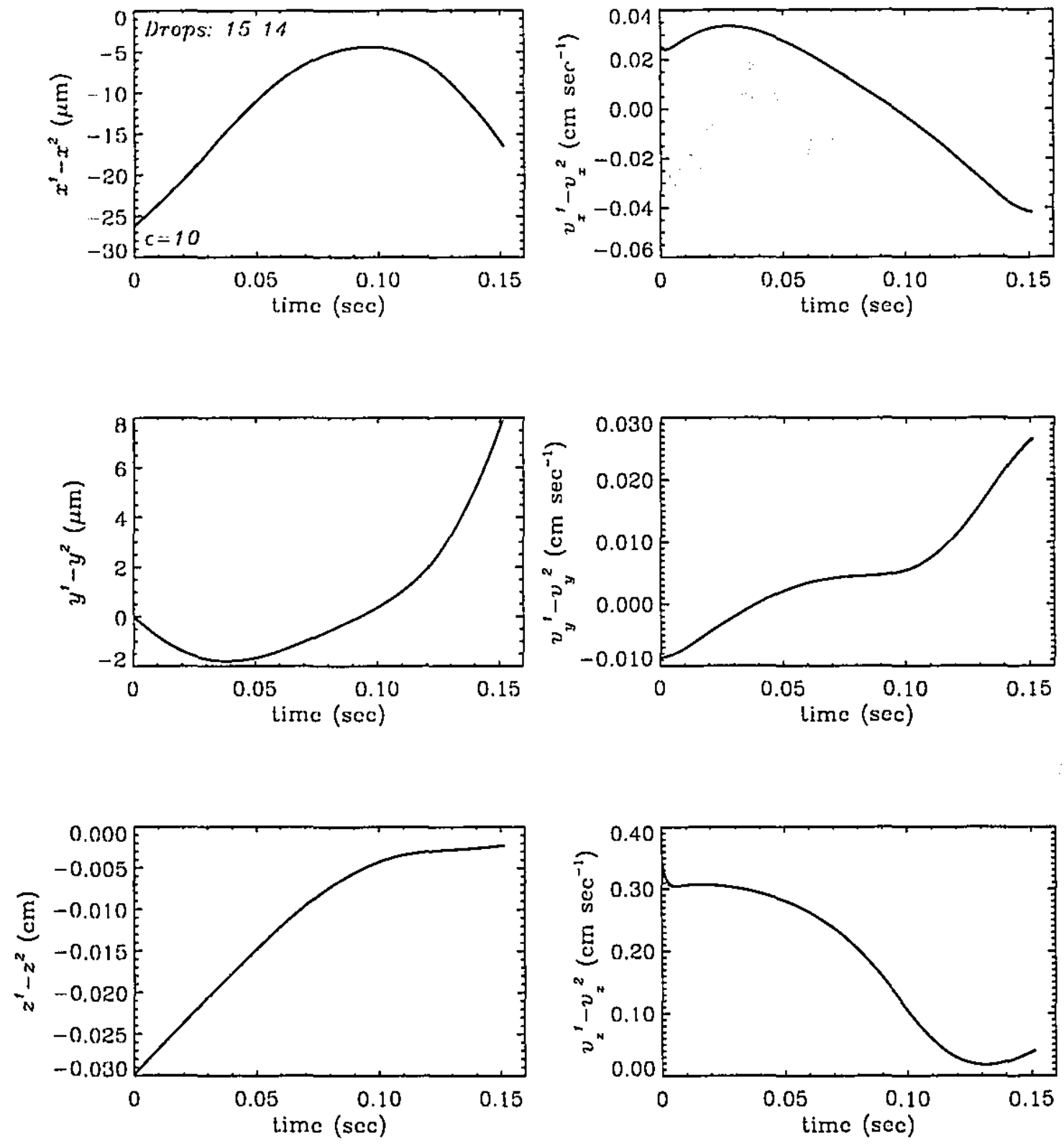

Fig. 5.9c The drop relative coordinates, $x^{1}-x^{2}, y^{1}-y^{2}$, and $z^{1}-z^{2}$, and the drop relative velocity components, $v_{x}^{1}-v_{x}^{2}, v_{y}^{1}-v_{y}^{2}$, and $v_{z}^{1}-v_{z}^{2}$ vs. time for the turbulent settling of 15 and $14 \mu \mathrm{m}$ drops and the rate of energy dissipation 10 $\mathrm{cm}^{2} \mathrm{sec}^{-3}$. The initial position of the smaller drop: $R=0.03 \mathrm{~cm}$ and $\theta=5^{\circ}$. The calculation results in a collision. 

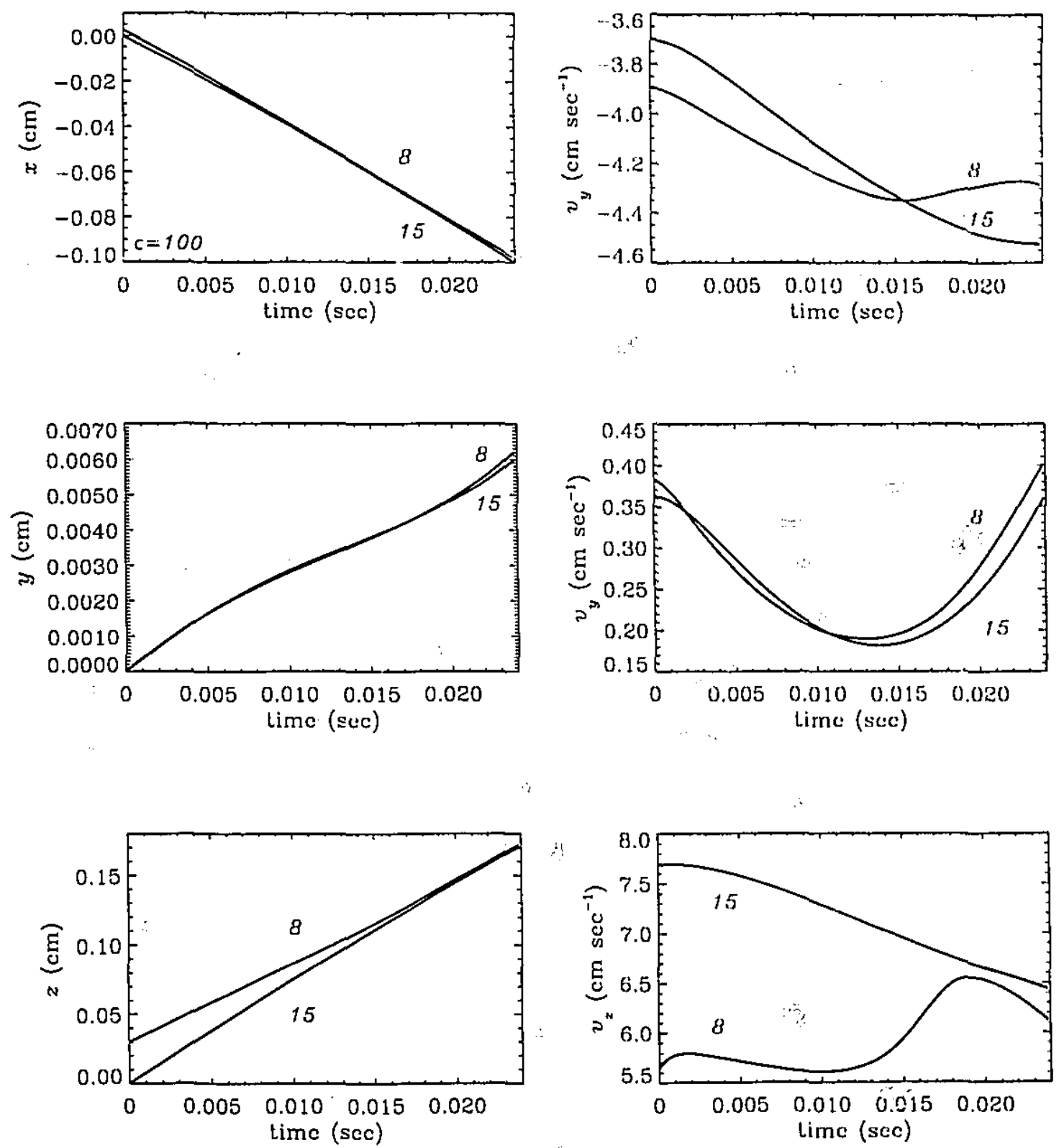

Fig. 5.10a The drop coordinates, $x, y$, and $z$, and the drop velocity components, $v_{x}$, $v_{y}$, and $v_{z}$ vs. time for the turbulent settling of 15 and $8 \mu \mathrm{m}$ drops and the rate of energy dissipation $100 \mathrm{~cm}^{2} \mathrm{sec}^{-3}$. The initial position of the smaller drop: $R=0.03 \mathrm{~cm}$ and $\theta=5^{\circ}$. Numbers indicate radii. The calculation results in a collision. 

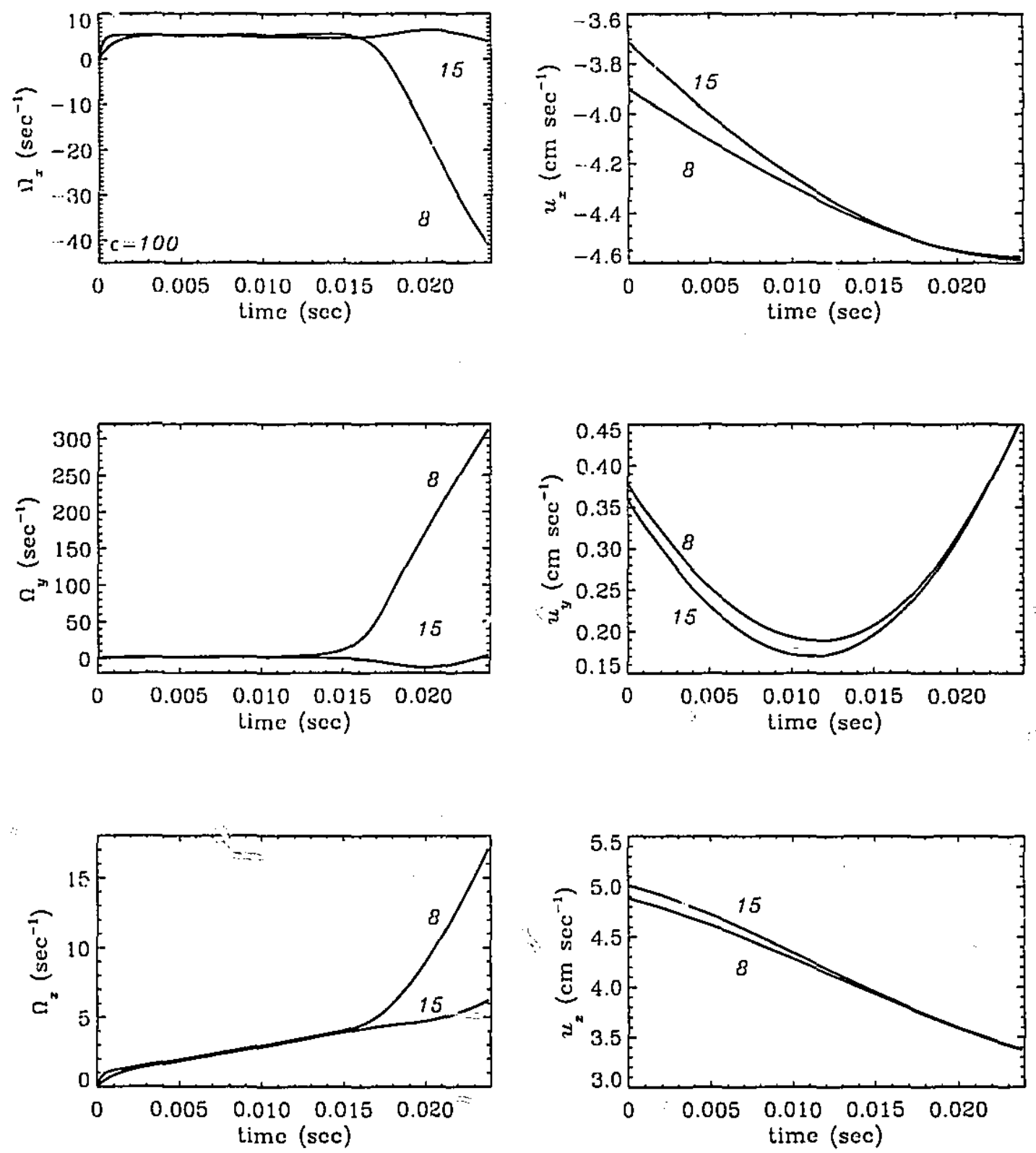

Fig: 5.10b The drop angular velocity components, $\Omega_{x}, \Omega_{y}$, and $\Omega_{z}$, and the turbulent velocity components, $u_{x}, u_{y}$, and $u_{z}$ at drop coordinates vs. time for the turbulent settling of 15 and $8 \mu \mathrm{m}$ drops and the rate of energy dissipation 100 $\mathrm{cm}^{2} \mathrm{sec}^{-3}$. The initial position of the smaller drop: $R=0.03 \mathrm{~cm}$ and $\theta=5^{\circ}$. Numbers indicate radii. The calculation results in a collision. 

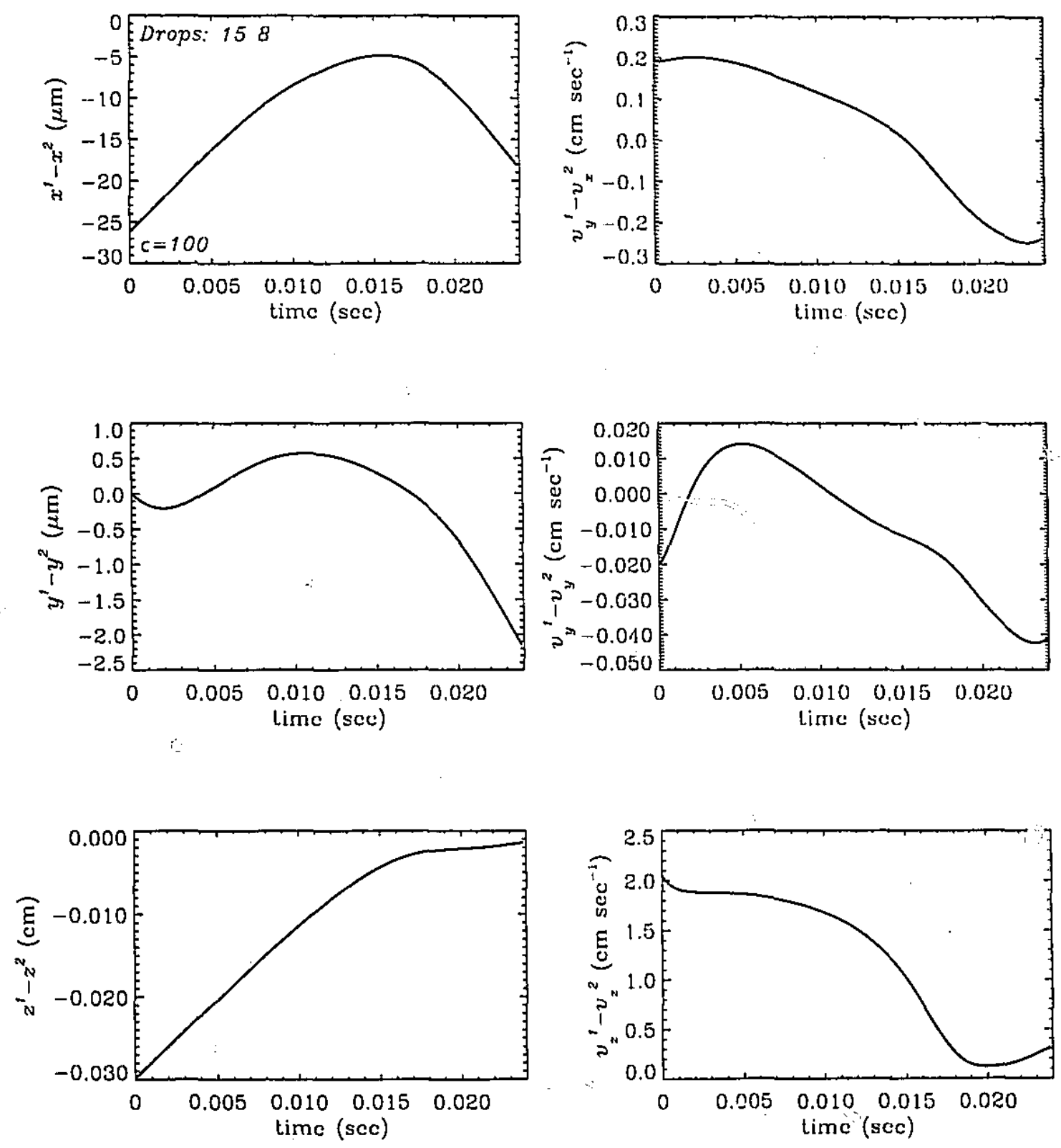

Fig. 5.10c The drop relative coordinates, $x^{1}-x^{2}, y^{1}-y^{2}$, and $z^{1}-z^{2}$, and the drop relative velocity components, $v_{x}^{1}-v_{x}^{2}, v_{y}^{1}-v_{y}^{2}$, and $v_{z}^{1}-v_{z}^{2}$ vs. time for the turbulent settling of 15 and $8 \mu \mathrm{m}$ drops and the rate of energy dissipation 100 $\mathrm{cm}^{2} \mathrm{sec}^{-3}$. The initial position of the smaller drop: $R=0.03 \mathrm{~cm}$ and $\theta=5^{\circ}$. The calculation results in a collision. 

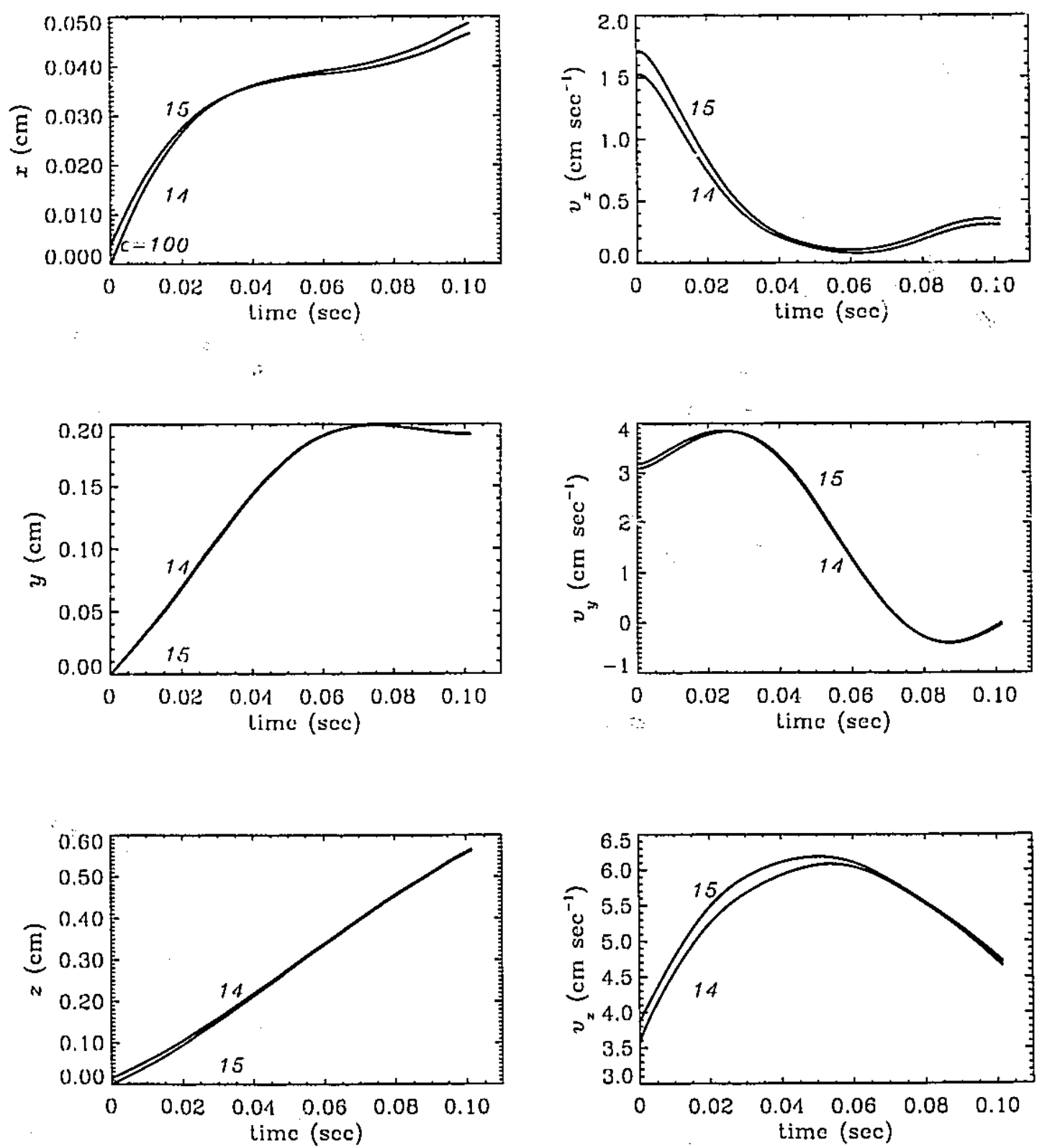

Fig. 5.11a The drop coordinates, $x, y$, and $z$, and the drop velocity components, $v_{x}$, $v_{y}$, and $v_{z}$ vs. time for the turbulent settling of 15 and $14 \mu \mathrm{m}$ drops and the rate of energy dissipation $100 \mathrm{~cm}^{2} \mathrm{sec}^{-3}$. The initial position of the smaller drop: $R=0.03 \mathrm{~cm}$ and $\theta=15^{\circ}$. Numbers indicate radii. The calculation results in a collision. 

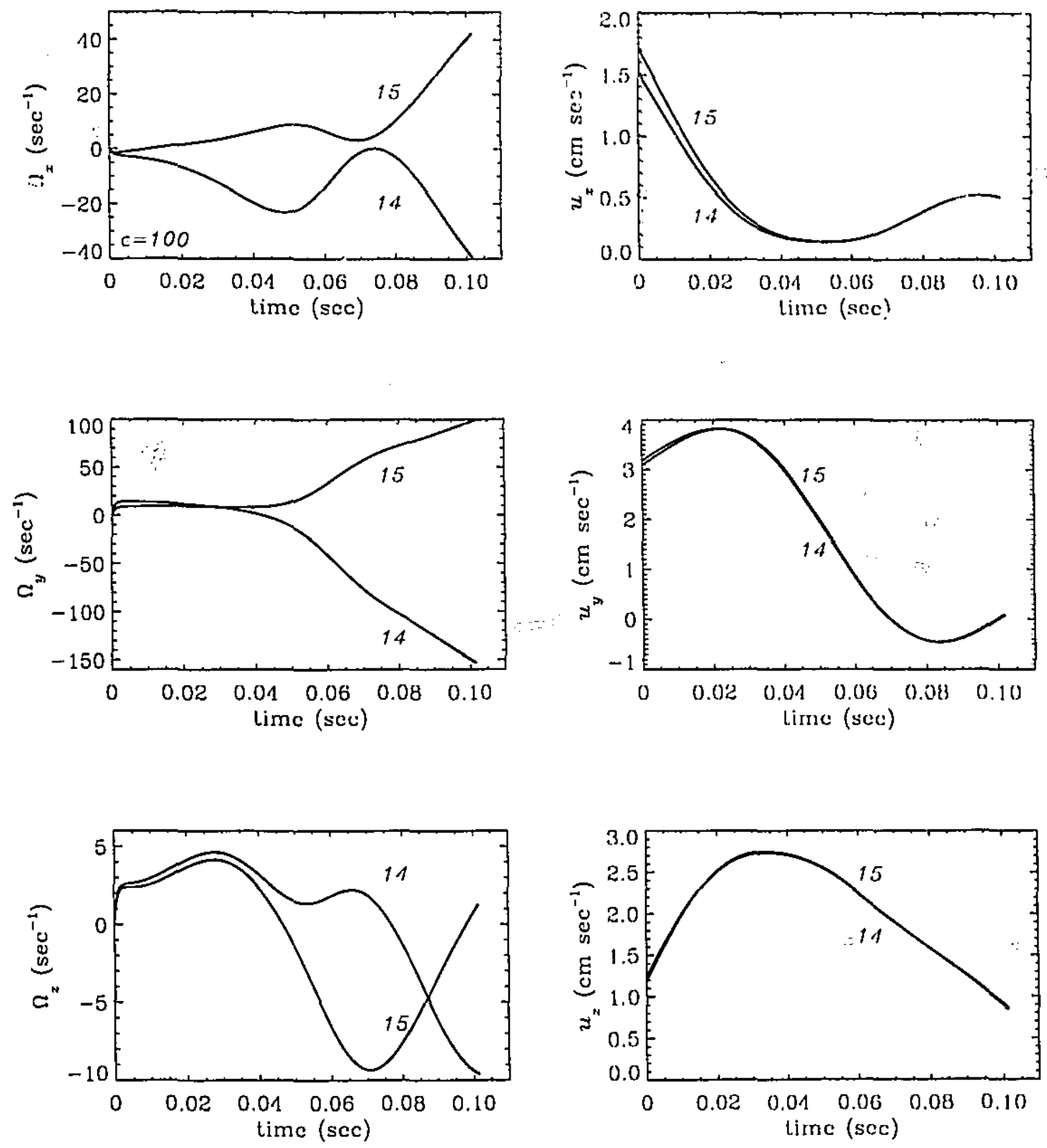

Fig. 5.11b The drop angular velocity components, $\Omega_{x}, \Omega_{y}$, and $\Omega_{z}$, and the turbulent velocity components, $u_{x}, u_{y}$, and $u_{z}$ at drop coordinates vs. time for the turbulent settling of 15 and $14 \mu \mathrm{m}$ drops and the rate of energy dissipation 100 $\mathrm{cm}^{2} \mathrm{sec}^{-3}$. The initial position of the smaller drop: $R=0.03 \mathrm{~cm}$ and $\theta=15^{\circ}$. Numbers indicate radii. The calculation results in a collision. 

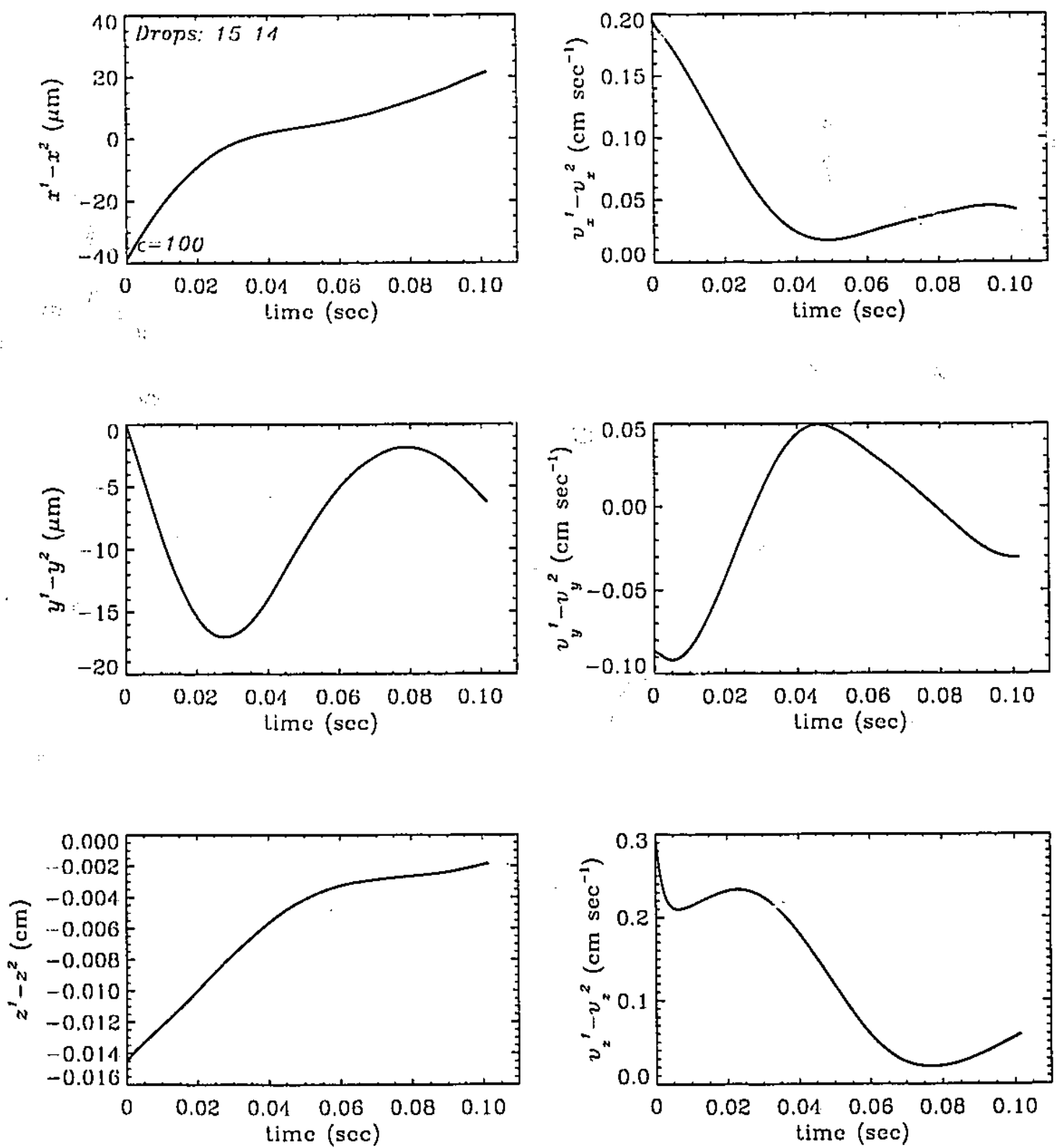

Fig. 5.11c The drop relative coordinates, $x^{1}-x^{2}, y^{1}-y^{2}$, and $z^{1}-z^{2}$, and the drop relative velocity components, $v_{x}^{1}-v_{x}^{2}, v_{y}^{1}-v_{y}^{2}$, and $v_{z}^{1}-v_{z}^{2}$ vs. time for the turbulent settling of 15 and $14 \mu \mathrm{m}$ drops and the rate of energy dissipation 100 $\mathrm{cm}^{2} \mathrm{sec}^{-3}$. The initial position of the smaller drop: $R=0.03 \mathrm{~cm}$ and $\theta=15^{\circ}$. The calculation results in a collision. 


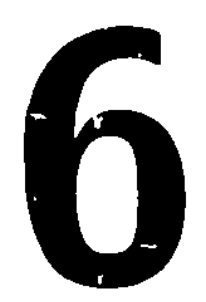

\section{Collision Kernels and Collision Efficiencies}

We have developed a conceptual model the purpose of which is to calculate collision rates. The inspiration for the model was drawn from the concept of the pure stochastic coalescence equation. With this equation as a starting point, we define collision kernels and collision efficiencies for turbulent flous.

\subsection{The Stochastic Coalescence Equation}

Since Telford [1955] introduced the coagulation equation (or kinctic equation) into cloud physics, it has been successfully apj)lied as a modelling tool. Cloud physicists tend to use the term stochastic coalescence equation (SCE hereaftcr). SCE describes the time evolution of drop spectra during the collision-coalescence process. Let us write the SCE in its discrete form (see for example Mason, 1971). (We use this form because of the convenience of notation-the general line of thinking cloes not change for the continuous version.)

$$
\frac{d n_{i}(t)}{d t}=\frac{1}{2} \sum_{j=1}^{i-1} K_{j, i-j} n_{j}(t) n_{i-j}(t)-\sum_{j=1}^{\infty} K_{i, j} n_{i}(t) n_{j}(t)
$$

where $n_{i}$ is the concentration of droplets ' $i$ ', and $K_{i j}$ is the collection kernel which represents the 'physics' of an unspecified yet process of collection of drops ' $j$ ' by drops ' $i$ '. Occasionally, however, questions have been asked whether the SCE is complete 
and what is the appropriate interpretation of the quantities occurring in the equation. (See for example Chin \& Neiburger [1972] or Gillespie [1972 and 1975].) Gillespie [1972] derives (6.1) from the first principles with only a few assumptions made on the way. He assumes that the probability that at the time instant $t$, there are exactly $N_{i}$ drops of type ' $i$ ' in the cloud does not depend on the number of drops of any other size, and that there are no collisions between identical drops. The spectrum predicted by the SCE, according to Gillespie, should be interpreted as an expected or averaged spectrum. Although, the form of the equation derived by Gillespie is identical to (6.1), in his later paper, Gillespie [1975] terms such a model as a pure stochastic model and provides the following physical interpretation of the collection kernel $A_{i j}=K_{i, j} / V$ ( $V$ is the cloud volume):

$$
\underbrace{A_{12} N_{2}}_{K_{12} n_{2}} \delta t=\mathcal{P}=\left\{\begin{array}{l}
\text { probability that any drop ' } 1 \text { ' will collect } \\
a \text { droplet ' } 2 \text { ' in time } \delta t .
\end{array}\right.
$$

For the laminar case this probability is

$$
\mathcal{P}=N_{2} \frac{\delta V_{\text {coll }}}{V}=n_{2} \delta V_{\text {coll }}
$$

where $\delta V_{\text {coll }}$ is the volume swept out by each drop ' 1 ' relative to each drop ' 2 ' (collection volume) in time $\delta t$. The standard expression for the collection volume for drops that do not interact hydrodynamically is

$$
\delta V_{\text {coll }}^{g}=\pi\left(r_{1}+r_{2}\right)^{2} w_{t} \delta t
$$

and for interacting drops

$$
\delta V_{\text {coll }}^{h}=\pi y^{2} w_{t} \delta t
$$

where $w_{t}=w_{1}-w_{2}$ is the difference of terminal velocities of drops ' 1 ' and ' 2 ', and $y$ is the distance from the centre of the collector drop representing the grazing trajectory, the, so called, impact parameter. A schematic view of two drops is depicted in Fig. 6.1. Using (6.2)-(6.5) one finds the respective kernels

$$
K_{12}^{g}=\pi\left(r_{1}+r_{2}\right)^{2} w_{t} \quad K_{12}^{h}=\pi y^{2} w_{t}
$$


If the collision efficiency is introduced as

$$
E_{12}=\frac{y^{2}}{\left(r_{1}+r_{2}\right)^{2}}
$$

the hydrodynamic kernel has the form

$$
K_{12}^{h}=\left[\pi\left(r_{1}+r_{2}\right)^{2} w_{t}\right] E_{12}
$$

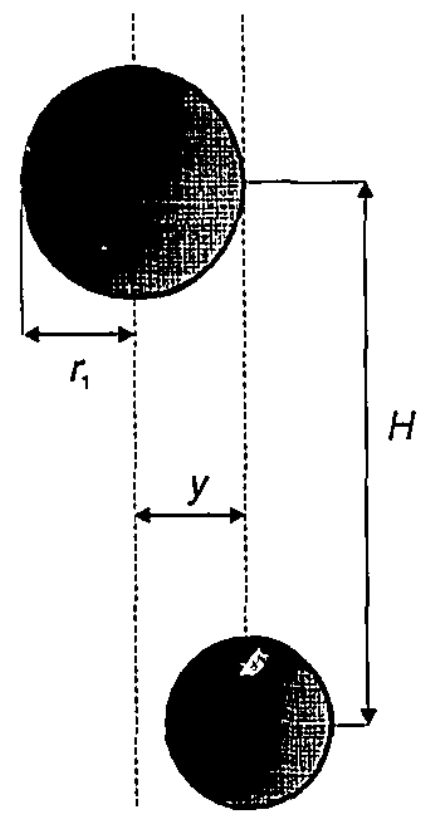

a) Before collision

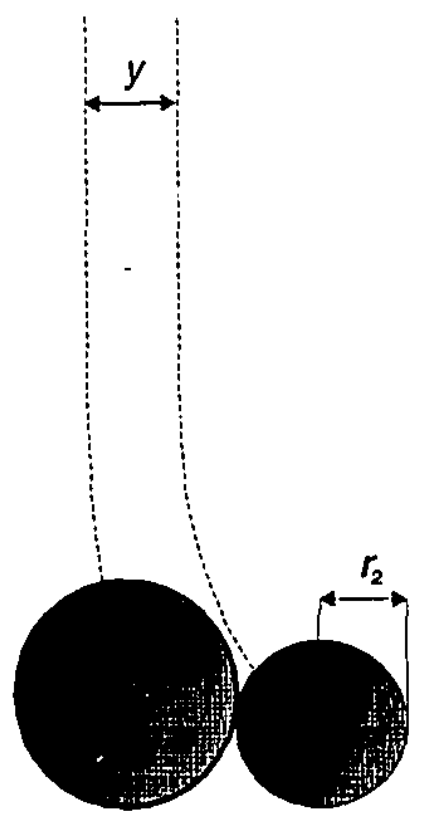

b) Collision

Fig. 6.1 Collision in a still fluid. Schematic representation of the grazing trajectory and the impact parameter $y$. 


\subsection{Turbulent Kernels and Collision Efficiencies}

\subsubsection{The Method of Collection Volume}

The extension of the concept of collection volume for the case of random (turbulent), ambient velocity field was introduced by de Almeida [1976]. The collection volume was envisioned by de Almeida as an infinite thin disc of thickness $w_{t} \delta t$. Here we modify the interpretation of some quantities in order to be consistent with the model of Gillespie [1975]. Because of symmetry, the disc is divided into infinitesimal rings, and the probability for each ring as described by (6.2)-i.e. the probability that a droplet ' 2 ' is found in the ring and subsequently collides with drop ' 1 '一is

$$
d \mathcal{P}=\underbrace{n_{2} V_{\text {coll }}(y)}_{\text {Probulility that droplet is in the ring }} \times \underbrace{P(y)}_{\text {Probability that droplet collides }}
$$

where $y$ is the distance from the centre of the disc (see Fig. 6.2). The collection volume of the ring is

$$
d V_{\text {coll }}=(2 \pi y d y) w_{t} \delta t
$$

Combining (6.9) and (6.10), and, subsequently, integrating results in

$$
\mathcal{P}=2 \pi n_{2} w_{t} \delta t \int_{0}^{\infty} y P(y) d y
$$

The respective collection kernel and collection efficiency are

$$
K_{12}=2 \pi w_{t} \int_{0}^{\infty} y P(y) d y=\left[\pi\left(r_{1}+r_{2}\right)^{2} w_{t}\right] E_{12}
$$

where

$$
E_{12}=\frac{2}{\left(r_{1}+r_{2}\right)^{2}} \int_{0}^{\infty} y P(y) d y .
$$

The above expression is an exact 'quotation' from de Almeida [1976]. In our opinion it should be modified as follows

$$
E_{12}=\frac{2}{\left(r_{1}+r_{2}\right)^{2}} \int_{0}^{\infty} y P(y ; H) d y
$$


where $H$ is the vertical distance between drops. This is because the probability $P$ depends significantly on $H$. (For example, the higher the value of $H$, the less likely a small droplet initially directly below a larger collector drop will collide.) The question which now arises is whether the entire value of the integral is independent of $H$. De Almeida did not mention that such a problem might exist. We do not attempt. to resolve it because a similar problem perplexes our method as well. We will rather address our own problem later in this chapter.

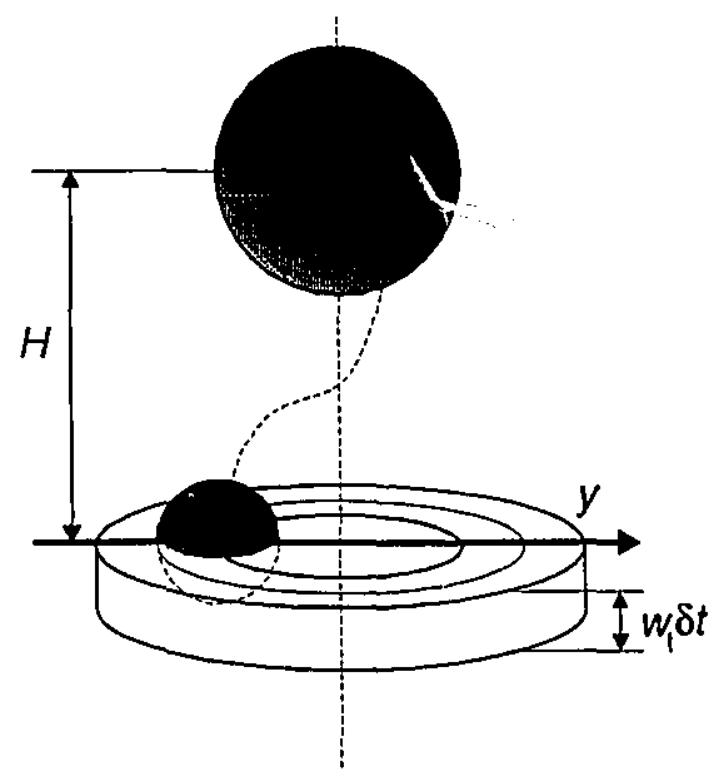

Fig. 6.2 Schematic view of the geometry for the method of collection volume.

\subsubsection{The Flux Method}

The method of collection volume described in the previous section is based on a cylindrical geometry. For a problem dominated by gravity this is a perfectly justified approach. However, as the turbulence gains more and more importance, (this happens when either turbulence becomes stronger or droplets are of similar sizes) spherical 
geometry may prevail. This is why we chose spherical geometry over cylindrical geometry. We follow the ideas of Smoluchowski [1916] for Brownian coagulation, and Saffman \& Turner [1956] for drop collisions.

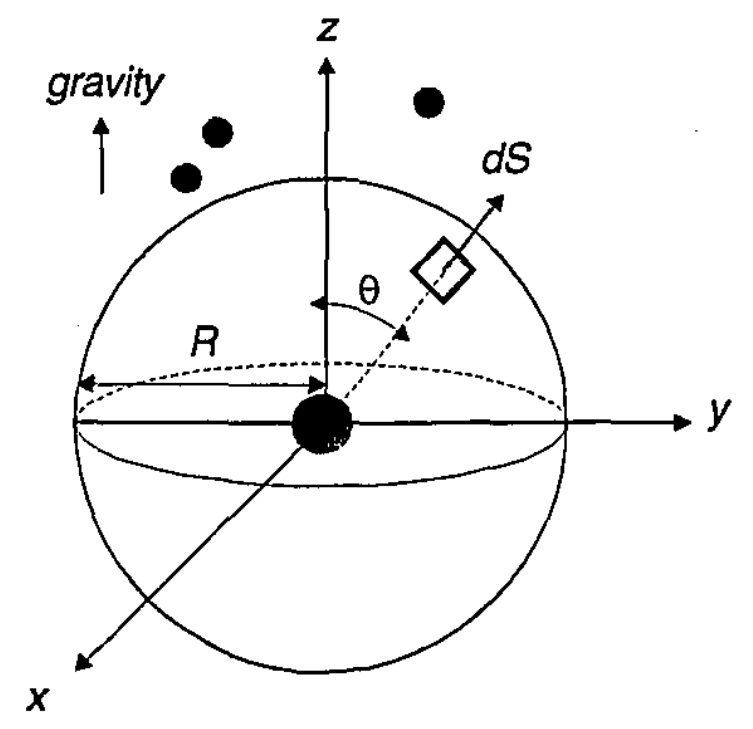

Fig. 6.3 Schematic view of the geometry for the flux method.

$\therefore$ Let us consider a flux of droplets ' 2 ' through the sphere of radius $R$ centred at the position of the collector drop ' 1 ' (see Fig. 6.3). The radius of the sphere is associated with the distance of interaction between drops. Note that the process under consideration-collisions between drop ' 1 ' and drops ' 2 '-is a statistically stationary one. The probability that a droplet ' 2 ' enters this sphere through the surface element $d \mathbf{S}$, and, subsequently, collides between the time instants $t$ and $t+\delta t$ is

$$
d \mathcal{P}=-\mathbf{j} \cdot d \mathbf{S} \delta t P(\mathbf{S}),
$$

where $\mathbf{j}$ is the average current of droplets ' 2 ', and $P(\mathbf{S})$ is the probability of collision for a droplet passing through the surface element $d \mathrm{~S}$. Because $\mathbf{j}=n_{2} \mathbf{w}$, w being the average relative velocity of drops ' 1 ' and ' 2 ',

$$
\mathcal{P}=-n_{2} \delta t \int_{\mathcal{S}} P(\mathbf{S}) \mathbf{w} \cdot d \mathbf{S} .
$$


Further, with the introduction of spherical coordinates,

$$
\mathcal{P}=2 \pi n_{2} \delta t R^{2} w_{t} \int_{0}^{\pi} P(\theta ; R) \cos \theta \sin \theta d \theta
$$

Note that $\mathbf{w} \cdot d \mathbf{S}=-w_{t} d S \cos \theta, d S=2 \pi R^{2} \sin \theta d \theta$, and, because of symmetry, $P(\mathbf{S})=P(\theta ; R)$. Finally, we arrive with the expressions for the collision kernel and collision efficiency as follows (we used (6.2) on the way)

$$
\begin{gathered}
K_{12}=\pi R^{2} w_{t} \int_{0}^{\pi} P(\theta ; R) \sin 2 \theta d \theta \\
E_{12}=\frac{R^{2}}{\left(r_{1}+r_{2}\right)^{2}} \int_{0}^{\pi} P(\theta ; R) \sin 2 \theta d \theta .
\end{gathered}
$$

The above formulation assumes that the radial 'in' component of the drop relat:ve velocity is equal to that resulting from the difference of drop terminal velocities $\left(w_{t} \cos \theta\right)$. If the need arises, e.g. if the radial 'in' component of the relative velocity depends on the turbulent velocity, the flux method can be modified, and new definitions of collision kernels and collision efficiencies can be formulated. (Still, we are considering the scenarios with the relative 'in' velocity being slightly perturbed in comparison to that resulting from the gravitational settling alone.) Thus, the flux of droplets ' 2 ' through the element of the sphere is

$$
\mathbf{w} \cdot d \mathbf{S}=-w^{i n} d S
$$

where $w^{\text {in }}$ is the absolute value of the average radial 'in' component, of the relative velocity. Further, the probability of collision is

$$
\mathcal{P}=2 \pi n_{2} \delta t R^{2} \int_{0}^{\pi} w^{i n}(\theta ; R) f(\theta ; R) P(\theta ; R) \sin \theta d \theta
$$

The new quantity which appears under the integral, $f(\theta ; R)$ represents the fraction of drops ' 2 ' for which the radial component of the relative velocity is directed toward 
the collector drop. (In the previous scenario $f$ would be 1 for $\theta<90^{\circ}$ or 0 otherwise.) Now the collision kernel is

$$
K_{12}=2 \pi R^{2} \int_{0}^{\pi} f(\theta ; R) w^{i n}(\theta ; R) P(\theta ; R) \sin \theta d \theta
$$

We can also define a somewhat artificial collision efficiency as

$$
E_{12}=\frac{2 R^{2}}{\left(r_{1}+r_{2}\right)^{2} w_{t}} \int_{0}^{\pi} f(\theta ; R) w^{i n}(\theta ; R) P(\theta ; R) \sin \theta d \theta
$$

Here we adapt the convention that the collision efficiency is defined on the basis of the collision kernel according to (6.8).

There is an obvious problem with the flux method which has to be addressed. Similarly to the method of collection volume, the dependence of the collision kernel on $R$ seems to obstruct the uniqueness of this quantity. This uncertainty can be easily resolved with the help of heuristic arguments. Let us not forget that the basic assumption behind the flux method is that the radius $R$ is the minimum distance beyond which the hydrodynamic interactions can be neglected. Therefore, the sensitivity of the obtained collision kernels for different values of $R$ needs to be evaluated for a small range of radii, in particular, between 10 and 20 radii of the collector drop. For the radius ratios and the rates of energy dissipation under consideration, the maximum angles $\theta$ with nonzero probability of collisions are of the order of $40^{\circ}$. Our calculations show that for $0^{\circ}<\theta<40^{\circ}$, both $f(\theta ; R)$ and $w^{i n}(\theta ; R)$ are independent of $R$ (in the mentioned above range: $10 r_{1}-20 r_{1}$ ). With the above in mind, we can safely assume that the flux of colliding drops represented by (6.22) depends on the number of colliding drops initiated at a particular sphere. This number, however, is constant. (Eacl colliding drop initiated at the sphere of a minimum considered radius must have been passing at an earlier instant of time through the sphere of the maximum considered radius because the relative drop velocity has always a nonzero gravitational component.) Thus, $K_{i j}$ is independent of $R$. 
It can be also shown that for the laminar case, the flux method converges to the method of collection volume. Let us rewrite (6.19) as follows

$$
E_{12}=\frac{R^{2}}{\left(r_{1}+r_{2}\right)^{2}} \int_{0}^{o_{\max }} P(\theta ; R) \sin 2 \theta d \theta
$$

where

$$
P(\theta, R)= \begin{cases}1, & \text { for } \theta \leq \theta_{\max } \\ 0, & \text { otherwise. }\end{cases}
$$

After simple calculation, the collision efficiency is

$$
E_{12}=\frac{R^{2} \sin ^{2} \theta_{\max }}{\left(r_{1}+r_{2}\right)^{2}}
$$

From Fig. 6.4, $y=R \sin \theta_{\max }$, and the collision efficiency takes the standard form of (6.7).

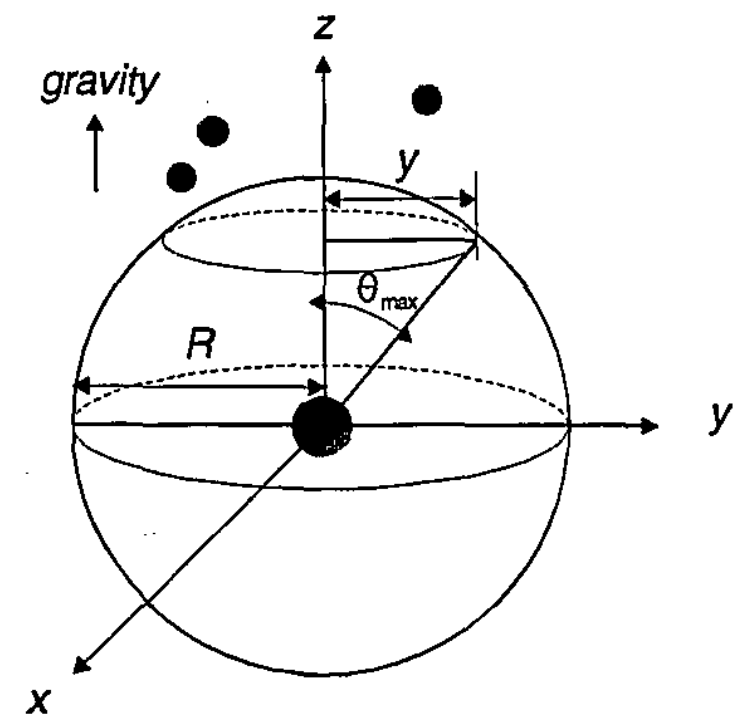

Fig. 6.4 Schematic vicw of the geometry for the flux method. The laminar case. 


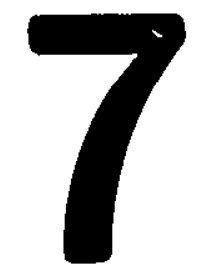

\section{Laminar Calculations}

Initially, laminar calculations were exclusively designed to test the methods which were later used in turbulent calculations. With time, however, the status of these tests was upgraded to become an integral part of the research. Both aspects of laminar calculations will be briefly discussed and also the methodology of laminar calculations in the literature will be outlined.

\subsection{An Overview of Methods of Calculating Collision Efficiencies in Still Air}

\subsubsection{The Superposition of Flow Fields}

Langmuir [1948] proposed that the motion of each of two drops be modelled by assuming that one drop is placed in the flow field generated by the other. With the above assumption, the equations of motion state

$$
\begin{aligned}
& m_{1} \frac{d \mathbf{v}_{1}}{d t}=m_{1} \mathrm{~g}-\left(\frac{C_{D}\left(\mathrm{Re}_{1}\right) \mathrm{Re}_{1}}{24}\right) 6 \pi \mu r_{1}\left(\mathbf{v}_{1}-\mathrm{u}_{2}\right) \\
& m_{2} \frac{d \mathbf{v}_{2}}{d t}=m_{2} \mathrm{~g}-\left(\frac{C_{D}\left(\operatorname{Re}_{2}\right) \mathrm{Re}_{2}}{24}\right) 6 \pi \mu r_{2}\left(\mathbf{v}_{2}-\mathbf{u}_{1}\right)
\end{aligned}
$$

where $\mathbf{v}$ denotes the drop velocities, and $\mathbf{u}$ the flow fields generated by drops; $C_{D}$ is the drag coefficient. In order to obtain the velocity field $\mathbf{u}$, the Navier-Stokes equations or the linearized Oseen equations are solved for each sphere in isolation. For example, 
Pearcey \& Hill [1956] utilized Goldstein's [1929] solution of linearized Oseen's [1910] equation; Shafrir \& Neiburger [1963] applied Jenson's [1959] method to obtain tine solution of the Navier-Stokes equation; and Lin \& Lee [1975] used a numerical solution of the Navier-Stokes equation [Lin \& Lee, 1973]. The superposition method does not capture exactly the nature of hydrodynamic interactions but, because of its ability to take the fluid inertia into account, is well suited to model the motion of large drops, up to the Reynolds number of the order of 100 [Pruppacher \& Klet,t, 1980].

\subsubsection{Direct Calculation of Hydrodynamic Forces}

The more accurate method of calculating drop trajectories involves a directi calculation of hydrodynamic forces (and torques.) The procedurc consists of two steps: the solution of the boundary value problem for two drops, and the subsequent: integration of the pressure field at drop surfaces to obtain forces and torques. For the Stokes approximation, the procedure was outlined in Chapter 3, except that for the still-air case the procedure is simpler because there is no external flow. Estimates of the collision efficiencies for Stokesian hydrodynamics were obtained by Davis \& Sartor [1969], and by Hocking \& Jonas [1970]. Further refinement to this method included the slip-flow effect as described in Chapter 2. The collision efficiencies with slip-flow were calculated by Davis [1969], and by Jonas [1972]. In the slip-flow appirdch, the dependence of the results on the minimum gap between surfaces was eliminated.

The Stokesian approximation is expected to yield satisfactory results for small drops, for which inertial effects can be neglected. If this is not the case, the effect, of fluid inertia needs to be addressed. Klett \& Davis [1973] proposed $\varepsilon$ method of solution to the boundary value problem with the Oseen-type corrections included. We present a brief outline of this method. In the Klett \& Davis approach, the flow 
around drops was governed by Oseen's equations for each sphere. (The solutions for velocity and pressure fields were represented by the superpositions of two flows.) The governing equations were solved with no-slip boundary conditions at the surfaces of the spheres. After obtaining the expressions for the forces in the usual way, by integrating the surface pressure, some corrections were introduced to the final force expressions. These were based on the work by Carrier [1953]. (The latter paper introduced correction to the Oseen-type drag on a sphere based on experimental data.) Klett \& Davis defined two Reynolds numbers for each drop, one based on the drop velocity and its radius ( $\mathrm{Re}$ ), and another based on the drop velocity and the distance between drops $\left(\mathrm{Re}^{\prime}\right)$. In the final solution, these Reynolds numbers were replaced in the following manner

$$
\operatorname{Re}=c \operatorname{Re} \quad \text { and } \quad \operatorname{Re}^{\prime}=c^{\prime} \operatorname{Re}^{\prime}
$$

where $c$ and $c^{\prime}$ are the Carrier constants

$$
c=1-0.08 \log (1+50 \mathrm{Re}), \quad \operatorname{Re} \leq 2 \quad \text { and } \quad c^{\prime}=0.43
$$

In the limit of zero Reynolds numbers (this is the range of special interest in the present research), the collision efficiencies of Klett \& Davis are higher than those obtained with the help of Stokesian approximation with no-slip boundary conditions [Davis \& Sartor, 1969]. Klett \& Davis explained that this is due to the force coefficients (scalar resistance functions in our preferred terminology) being incorrect at close distances. However, they noted too that the Stokesian solution is also incorrect for close separations. Although both statements are correct, the sources of the problems at small separations are different in the two methods. The Klett \& Davis method of solution does not resolve well the flow for close separations. The Stokesian formulation is not valid when the assumption of continuity breaks down due to molecular forces. While the former method underestimates the hydrodynamic forces 
(and overestimates the collision efficiencies), the latter overestimates the hydrodynamic forces (and underestimates the collision efficiencies). The built-in error of each of these methods is difficult to assess.

As far as the results for larger drops (with important inertial effects) are considered the method of Klett \& Davis performed well. The experimental verification will be discussed later.

\subsubsection{The Choice of the Hydrodynamic Representation}

In Chapters 3 and 5, we clearly stated that the method of modelling of lydrodynamic; interactions applied in the present work is that of direct, calculation of hydrodynamic forces with no-slip boundary conditions. Is it the best possibly approach? As far as the feasibility of calculations is concerned-yes-this seems to be the best way of proceeding. In this section, we will try to convince the reader that from the point of view of the accuracy of a representation the method chosen in the present research if not the best is, at least, adequate for the problem at hand. A number of experiments have been conducted in order to verify the theoretical collision efficiencies in still air [Woods \& Mason, 1964; Picknett, 1967; Jonas \& Goldsmith, 1972; Kranogorskaya \& Neizvestnyy, 1973; and Kobzunenko \& Neizvestnyy, 1980.] In the last of these papers, a very comprehensive summary of the theoretical and experimental data was performed. Kobzunenko \& Neizvestnyy [1980] divided drops into three size ranges with the radius of the collector drop as the criterion: 1 . $38-100$ $\mu \mathrm{m}, 2$. 25-38 $\mu \mathrm{m}$, and 3. $12-25 \mu \mathrm{m}$. It was found that in the first range, the method of superposition is applicable, in the second range, the method by Klett, \& Davis performs the best, while in the third range, the Stokesian approximation seems to be quite appropriate. In particular, in the third range, both the slip-flow approach 
[Davis, 1969; Jonas, 1972], and the no-slip approach with $\varepsilon=8 \times 10^{-3}$ [Davis \& Sartor, 1969; Hocking \& Jonas, 1970] perform equally well.

The collector droplets under investigation are in the $10-20 \mu \mathrm{m}$ range. Therefore, we feel that the use of the Stokesian approach with no-slip boundary conditions is well justified. In Fig. 7.1, there is presented a comparison of the collision efficiencies for Stokesian approximation obtained by other researches with those obtained in the present research for $\varepsilon=10^{-3}$. (The vertical distance between drops used in the calculation is $0.7 \mathrm{~cm}$.) As expected, our collision efficiencies are lower than those obtained with the slip-flow theory [Jonas, 1972] and higher than those with no-slip and $\varepsilon=10^{-4}$ [Hocking \& Jonas, 1970]. The reader may be puzzled by the fact that our collision efficiencies for $30 \mu \mathrm{m}$ collector drops in the middle range of radius ratios are somewhat lower than those obtained by Hocking \& Jonas [1970]. The similar tendency-a flatness of the collision efficiency curve in the middle of the range of radius ratios can be also seen in our collision efficiency curve for the $20 \mu \mathrm{m}$ collector drop. We performed a simple test in order to clarify this issue. While modelling drop trajectories in order to determine the impact parameters, it is important to choose the initial separation large enough so that drops do not interact hydrodynamically. (This distance, for the still-fluid case, corresponds to the vertical separation between drops-horizontal separations are much smaller.) Researchers seldom specify this vertical separation, and the mutual reader-author understanding is that these vertical distances are sufficiently large. In the paper by Hocking \& Jonas [1970], the mentioned distance is described as "large". We calculated the collision efficiencies for the $30 \mu \mathrm{m}$ collector drop for $\varepsilon=10^{-4}$ and different initial vertical separations. Our results converged to those by Hocking \& Jonas for the vertical separations of the order of 20 collector drop radii. Although, from the point of view of the dimensional analysis, the interactions between drops decrease quickly for larger distances than 
that of 10 collector drop radii, still, if the time of interaction is prolonged, these weak interactions cannot be neglected.

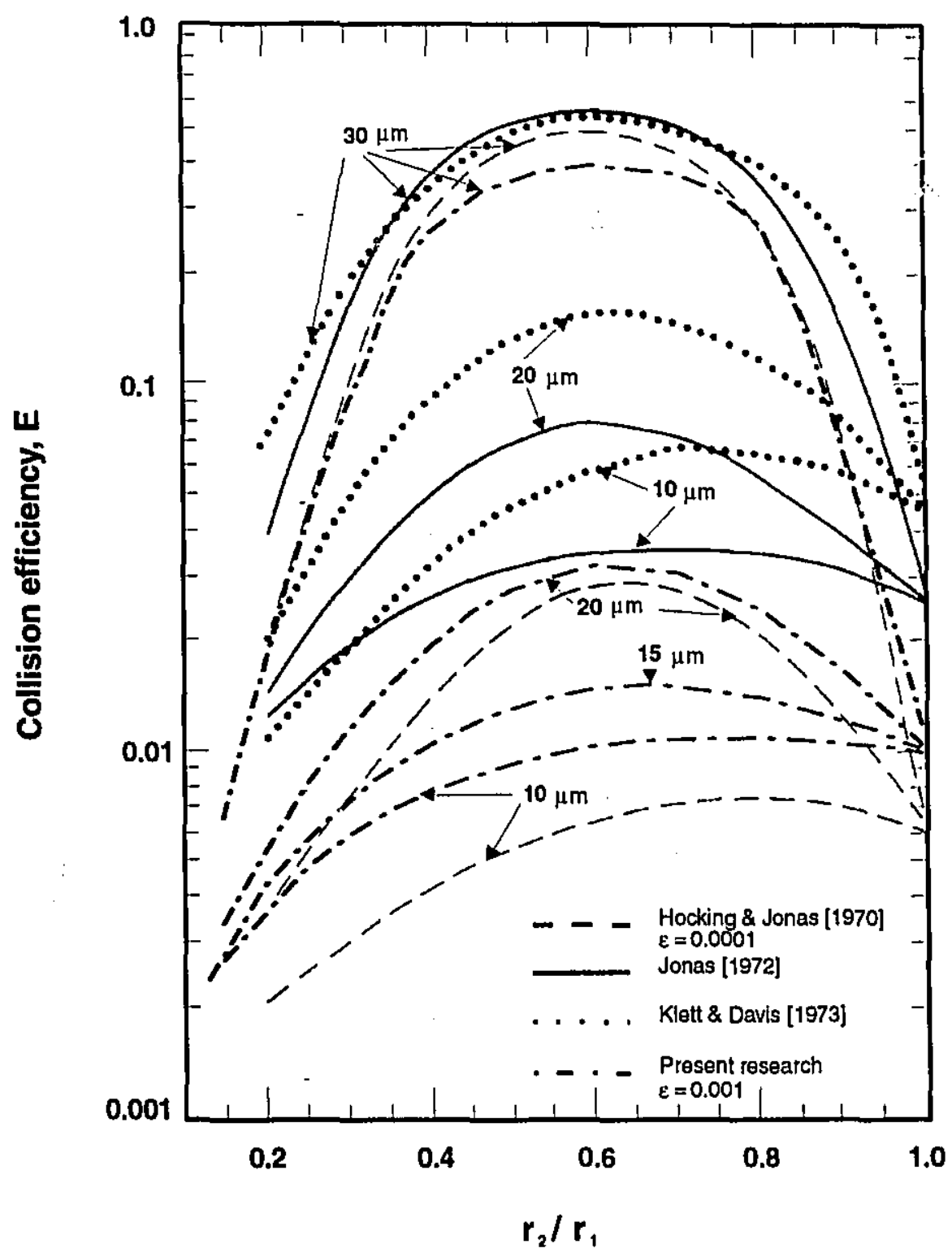

Fig. 7.1 Collision efficiencies in still fluid calculated with the Stokes approximation and with the Oseen approximation in the limit of zero Reynolds number. 


\subsection{The Case of a Linear Shear}

This and the following section are dedicated to the calculation of collision efficiencies for gravitational settling of drops embedded in simplified types of laminar flows. The motivation for these numerical experiments was twofold: to check the flux model as well as to gain some insight into how simple steady flows with nonzero space derivatives of the velocity can influence collision efficiencies. In particular, we examined a linear shear flow and a two-dimensional deformation field with the hope that the conclusions might be applicable to real turbulent flows. Although, in a real turbulent field, both elements vary in a random manner, the examination of the sensitivity of collision efficiencies in steady laminar flows may provide some insight into turbulent cases.

The flux method can be easily exteilded to the case of a linear shear (see Fig. 7.2). The only major difference is that related to the lack of axial symmetry, and, as a consequence, the integration with respect to $\phi$ cannot be as easily performed. Let us consider a linear shear in the form

$$
L=\frac{\partial u_{x}}{\partial z}
$$

The collision kernel is now

$$
K=R^{2} \int_{\phi_{1}}^{\phi_{2}} d \phi \int_{0_{1}(\phi)}^{\theta_{2}(\phi)} w^{i n}(\phi, \theta ; R) \sin \theta d \theta
$$

where the radial 'in' component of the velocity is composed of the gravitational and shearing parts as follows

$$
w^{i n}=w_{t} \cos \theta+L z \sin \theta \cos \phi=w_{t} \cos \theta+L R \sin ^{2} \theta \cos \phi .
$$

The limits of integration determine that area of the sphere where the colliding smaller drops originated at the initial time instant. These limits of integrations were obtained 
with the help of our hydrodynamic model described in Chapters 3 and 5. The value of $R$ was chosen similar to the value for settling in a still flow, i.e. equal to $0.7 \mathrm{~cm}$. Although the calculation is laminar, in order to make comparisons, we assume that. the linear shear is equal to the average turbulent shear [Taylor, 1935]

$$
L=\sqrt{\left(\frac{\partial u_{x}^{t u r b}}{\partial z}\right)^{2}}=\sqrt{\frac{2 \epsilon}{15 l}} .
$$

For $\epsilon=100 \mathrm{~cm}^{2} \mathrm{sec}^{-3}$, this shear is equal to $9.3 \mathrm{sec}^{-1}$. Two pairs of drops were considered, 10 and $9 \mu \mathrm{m}$, and 15 and $9 \mu \mathrm{m}$. No change of collision efficiency when compared with the still-air case was observed.

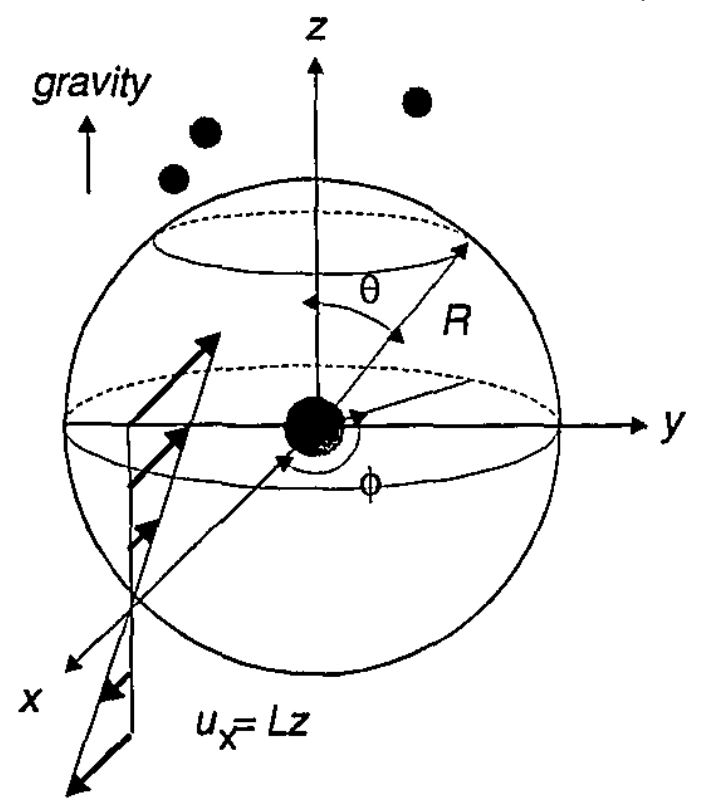

Fig. 7.2 The flux method for the case of a laminar shear flow.

Our results are in accord with the numerical calculation by .Jonas \& Goldsmith [1972]. (Jonas \& Goldsmith performed a series of experiments which showed a significant increase of collision efficiencies of 10-15 $\mu \mathrm{m}$ collector drops for $9 \mu \mathrm{m}$ drops. However, their numerical calculation did not show such an effect.) Our results can be compared with the experimental value of 8 and $10 \mathrm{sec}^{-1}$ of turbulent shear in the Jonas \& Goldsmith experiment. 


\subsection{The Case of a Two-Dimensional Deformation Field}

Now, let us consider a pure two-dimensional deformation field (see Fig. 7.3). Let, us also give it the magnitude of the average strain for a turbulent flow

$$
\begin{gathered}
D=\sqrt{\left(\frac{\partial u_{z}^{\text {turb }}}{\partial z}\right)^{2}}=\sqrt{\frac{\epsilon}{15 \nu}} \\
\frac{\partial u_{z}}{\partial z}=-D \quad \text { and } \quad \frac{\partial u_{x}}{\partial x}=D .
\end{gathered}
$$

The strain corresponding to $\epsilon=100 \mathrm{~cm}^{2} \mathrm{sec}^{-3}$ is $6.7 \mathrm{sec}^{-1}$. Note that in the method of fluxes there are now three constituents of the radial 'in' component of the velocity:

$$
w^{i n}=w_{t} \cos \theta+D R \cos ^{2} \theta-D R \cos \phi \sin ^{2} \theta
$$

The collision kernel was expressed in the same way as for the linear shear case, and the procedure of obtaining the limits of integration were the same as those for a linear shear. The collision efficiencies for the collector drops in the radius range 10-30 $\mu \mathrm{m}$, and $9 \mu \mathrm{m}$ collected drops are plotted in Fig. 7.4. Recall that in order to obtain the collision efficiency, the collision kernel is divided by $\pi w_{t}\left(r_{1}+r_{2}\right)^{2}$. In Fig. 7.4, the highest relative increase of the collision efficiency can be observed for drops of radii 10 and $9 \mu \mathrm{m}$. Further, for larger collector drops, there is almost no increase. Then, again, for collector drops of radii larger than $25 \mu \mathrm{m}$, a steady relative increase of the collision efficiency with respect to the still-air values is present. In summary, the effect of a deformation field seems to be more pronounced for small $(<0.4)$ and large ( $>0.7)$ radius ratios. Incidentally, we have observed a similar tendency in our calculations with the realistic turbulent field. These will be shown in Chapter 9 . 


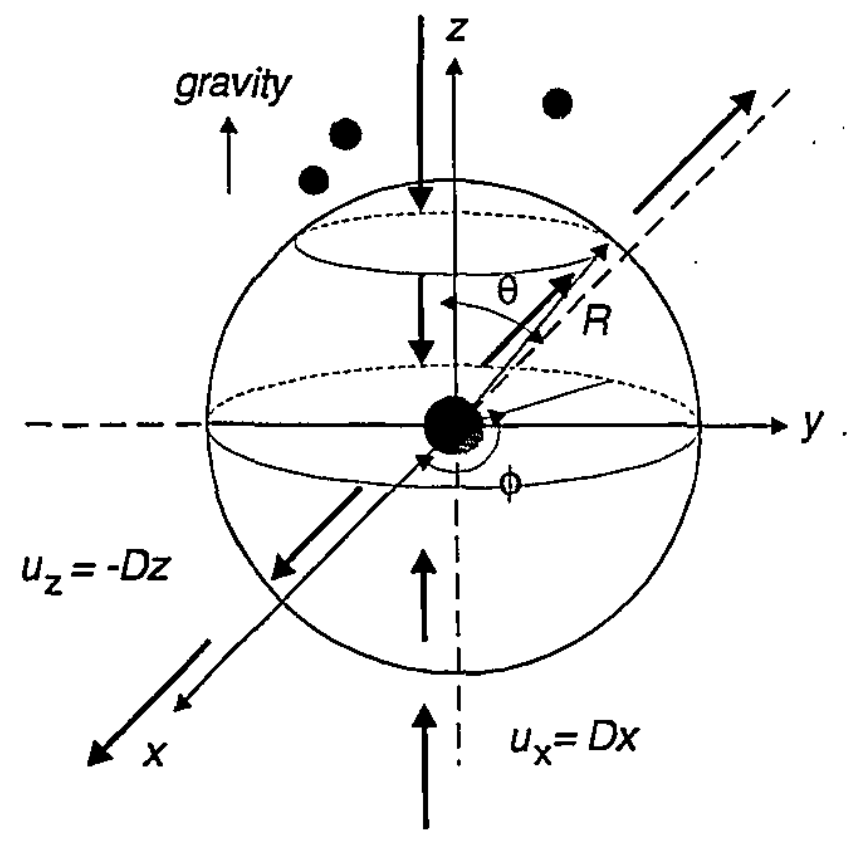

Fig. 7.3 The flux method for the case of a twodimensional deformation field.

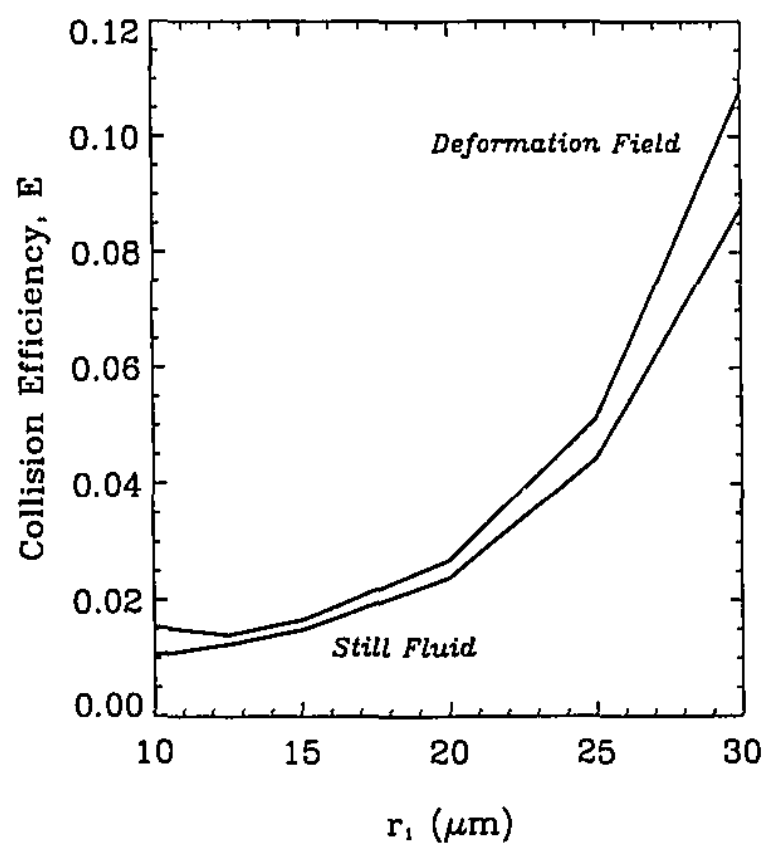

Fig. 7.4 Collision efficiencies for $9 \mu \mathrm{m}$ drops for still flow and deformation field. 


\subsection{Collision Efficiency for Almost Equal Drops}

Drops of equal (or almost equal) sizes represent a different category than drops of markedly different sizes. This is because gravity, the dominant factor for the latter, is no more a major factor determining the character of the motion. Time and scale of interaction of such drops increase to infinity. Therefore, the inertia of the fluid, which is neglected for very small droplets, may become increasingly important. The method of Klett \& Davis [1973] attempted to include the fluid inertia. Their results, however, are too high. (Recall that we consider very small drops of radii equal or smaller than $20 \mu \mathrm{m}$.) In a recent paper, Böhm [1992] applies the concept of a boundary layer around a drop in order to formulate a general hydrodynamic theory. The results are in a reasonably good agreement with the collision efficiencies presented by Hall [1980] for drops of different sizes (at least in a qualitative sense). The two sets of collision efficiencies, however, contradict each other for similar drops and small collector drops (radii smaller than $25 \mu \mathrm{m}$ ). (Note that the paper by Hall [1980] assemblies the most reliable collision efficiency curves available at that time.) The disagreement in the two sets of collision efficiencies concerns the increase or absence of increase of the collision efficiencies as the radius ratios tend to one. In other words, in the boundary layer approach, the collision efficiencies of very small, almost identical drops increase dramatically due to the effect of the fluid inertia while in the method of direct calculation of hydrodynamic forces the collision efficiencies do not reveal such a behaviour. Böhm compares his results with the available experimental data. The comparison is favourable, but again, for drops larger than those of interest here. There are no experimental data for smaller drops. Also, from the theoretical point of view, the extension of the boundary layer approach to very small drops is incorrect. The derivation of the boundary layer equations (for the axisymmetrical shape of the body in this particular case) requires that the drop radius be much greater than the 
thickness of the boundary layer. If this was so, the following could be written (see for example Schlichting, 1968)

$$
\frac{\delta}{r} \sim \sqrt{\frac{1}{\operatorname{Re}}}
$$

where $\delta$ is the boundary layer thickness, $r$ is the drop radius, and Re is the Reynolds number as defined in Chapter 2. For a $10 \mu \mathrm{m}$ radius drop, the above condition gives $\delta / r \propto 11$ not a small number at all. Thus, the application of the boundary value approach was incorrect at the onset.

Clearly then, even if the consensus has been reached in the literature [Böhm; 1992] that the collision efficiencies for large collector drops increase steeply as the radius ratio tends to one, for small collector drops the question seems to be still open.

Let us now return to our turbulent case. With the present version of our mode', the calculation of the turbulent collision kernels for small, almost identical drops cannot be accomplished in the same way as for drops markedly different in size. (Without the 'support' of gravity, the number of collisions is too low to obtain any statistically significant results.) Therefore, we are interested to obtain the estimates of the turbulent collision rates for equal sized drops on the basis of the assumption that, hydrodynamic interactions do not influence the relative separation between drops. In the 'laminar still-air terminology', the latter is equivalent to the collisional crosssection being equal to the geometrical cross-section, and, consequently, the collision efficiency equal to unity. If there are no hydrodynamic interactions, we could use the flux model, and calculate $w^{\text {in }}$ at $R=r_{1}+r_{2}$-the probability of collision, $P(\theta ; R)$, assumed one. Theoretically, we could perform a turbulent calculation which will show us whether or not the above hypothesis is true. However, due to large statistical uncertainties resulting from the small number of collisions in the turbulent calculations, the numerical experiment will not be conclusive. Nevertheless, we can apply the deformation field, as defined in Section 7.3, to calculate the collision kernels for 
equal drops for both with and without hydrodynamic interactions. The results of the above experiments for two pairs of drops of radii 15 and $15 \mu \mathrm{m}$, and 15 and $9 \mu \mathrm{m}$ are presented in Table 7.1. All collision kernels were obtained for $R=0.7 \mathrm{~cm}$.

TABLE 7.1

Collision Kernels for Two-Dimensional Deformation Field

\begin{tabular}{cccc}
\hline $\begin{array}{c}\text { Radius } r_{1} \\
(\mu \mathrm{m})\end{array}$ & $\begin{array}{c}\text { Radius } r_{2} \\
(\mu \mathrm{m})\end{array}$ & $\begin{array}{c}\text { Hydrodynamic } \\
\text { Interactions }\end{array}$ & $\begin{array}{c}\text { Collision Kernel } \\
\left(\mathrm{cm}^{3} \mathrm{sec}^{-1}\right)\end{array}$ \\
\hline 15 & 15 & Yes & $3.38 \times 10^{-8}$ \\
15 & 15 & No & $3.01 \times 10^{-7}$ \\
15 & 9 & Yes & $5.13 \times 10^{-7}$ \\
15 & 9 & No & $3.26 \times 10^{-5}$ \\
\hline
\end{tabular}

Although, for identical drops, the collision kernels are not equal for the interacting and non-interacting drops still, the ratio of these two kernels is significantly higher for equal than for unequal drops ( 0.11 and 0.02 respectively.) Thus, with the help of this simple experiment we have shown that for drops of almost identical sizes embedded in a deformation field, the effect of hydrodynamic interactions on collision kernels becomes substantially less important than for drops of different sizes. We might, therefore, assume that if we werc to decide to estimate the collision kernels for equal drops in a real turbulent field, neglecting hydrodynamic forces would result in an overprediction by a factor of about 9 . 


\section{8}

\section{Turbulent Calculations}

We are ready to present the calculation of the turbulent collision efficiencies at. last. In this chapter, we describe the details concerning the use of the model designed to estimate collision kernels and collision efficiencies. We also show the results of the tests designed to validate our model. Finally, we outline the method of estimating errors.

\subsection{The Operational Details of the Model}

In Chapters 3, 4, 5, and 6, we described the structural elements of our model. In particular, in Chapter 6, we introduced a statistical approach which allows us to calculate collision kernels for known values of the probability of collision as a function of the azimuthal angle, $\theta$. The role of our dynamic model was to facilitate the calculation of this probability. It was accomplished through multiple repetitions of the same experiment for different realizations of the turbulent velocity field. In each experiment, the large drop was placed at, the origin and the small drop on the surface of the sphere of radius $R$ centred at the origin, in particular at $(R, \theta, \phi)$. The coordinate $\theta$ was varied while the coordinate $\phi$ was always assumed to be $0^{\circ}$ (recall symmetry in $\phi$ ). Then, the initial value problem for two drops was carried out up to the time instant when either droplets collided or the large drop passed the small one, and there was no chance for the occurrence of collision. (The details of trajectory calculations were given in Chapter 5.) After a sufficient number of trajectories were 
examined, the probability of collision was estimated as

$$
P(\theta ; R)=\frac{N_{\text {coll }}}{N_{\text {exp }}}
$$

where $N_{\text {coll }}$ is the number of collisions, and $N_{\text {exp }}$ the total number of the performed experiments. The subsequent integration of $P(\theta ; R)$ allowed us to obtain the collision kernels and collision efficiencies as follows

$$
\begin{gathered}
K=\pi R^{2} w_{t} \int_{0}^{\theta_{\max }} P(\theta ; R) \sin 2 \theta d \theta \\
E=\frac{R^{2}}{\left(r_{1}+r_{2}\right)^{2}} \int_{0}^{\theta_{\max }} P(\theta ; R) \sin 2 \theta d \theta .
\end{gathered}
$$

Before, however, the numerical values could be obtained a number of choices had to be made. The list of such tasks follows.

1. The choice of radius $R$.

2. The truncation of turbulence modes which do not influence the relative motion of drops.

3. The number and spacing of angles $\theta_{i}$ for the integration.

4. The value of $\theta_{\max }$.

5. The number of experiments for each $\theta_{i}$.

We will comment on each of the above items. The choice of $R$ was determined by two factors, the strength of the hydrodynamic interactions, and the effectiveness of calculations. (The latter required that a reasonably sufficient number of collisions was encountered-this number of collisions per number of experiments decreases with $R$ ) Unlike the case of a still fluid, the turbulent case is not sensitive to weak interactions between drops. These can be neglected because at large separations, the relative displacements of drops due to the hydrodynamic interactions are much smaller than those due to turbulence. A more rigorous proof will be given later in this chapter. In the majority of calculations $R=20 r_{1}$. Only in situations in which the relative 
displacement of drops due to turbulence is much larger than the drop radii, e.g. for drop radius ratios of the order or greater than 0.9 , and for the rate of energy dissipation 10 and $100 \mathrm{~cm}^{2} \mathrm{sec}^{-3}, R=10 r_{1}$.

The second issue on our list was dictated by the necessity to limit computer time. Different drop pairs are characterized by different time and length scales of interaction. Therefore, the range of the Fourier modes which actively participate in binary drop interactions vary. There is no need to include those modes which have time and length scales much larger than the scales of interaction. This is because they do not contribute to the relative displacement but merely impose a background velocity field, identical for both drops. The practical criterion which we applied was that all the scales larger than 50 times the scales of interaction were truncated. This rule was relaxed for the situations with very short time scales of interaction-of the order or shorter than the Kolmogorov time scale. In such fields drops do not sce time variation because the frequencies associated with the wavelengths which affect the motion of the drops are much smaller than the inverse of the time scale characterizing the two-drop problem. Therefore, the strict implementation of the above rule would result in the rejection of all the modes. Sensitivity tests for the truncations of the Fourier modes will be presented in Section 8.3.2.

All the decision-making procedures in items 3,4 and 5 were based on a subjective judgment. Initially, we started with $\theta_{i}$ uniformly distributed in what we anticipated to be the range of nonzero probability of collision. Then, looking at the curve $P(\theta) \sin 2 \theta$ vs. $\theta$ (rather than $P(\theta)$ vs. $\theta$ ), decisions concerning addition of extra points were made. This type of a subjective judgment worked well for the 'weak-turbulence' cases (smooth curves) as opposed to 'strong-turbulence' cases (noisy curves). In the latter category, error analysis was used in order to decide whether more values of $\theta$ needed to be examined, but, again, the final decisions were 
made in a rather arbitrary way. In order to illustrate what we mean by a 'weak-' and a 'strong-turbulence' case, in Fig 8.1, there are presented curves of $P(\theta)$ and $P(\theta) \sin 2 \theta$ vs. $\theta$ for both cases. The integration limit $\theta_{\max }$ was determined by the vanishing probability of collision.
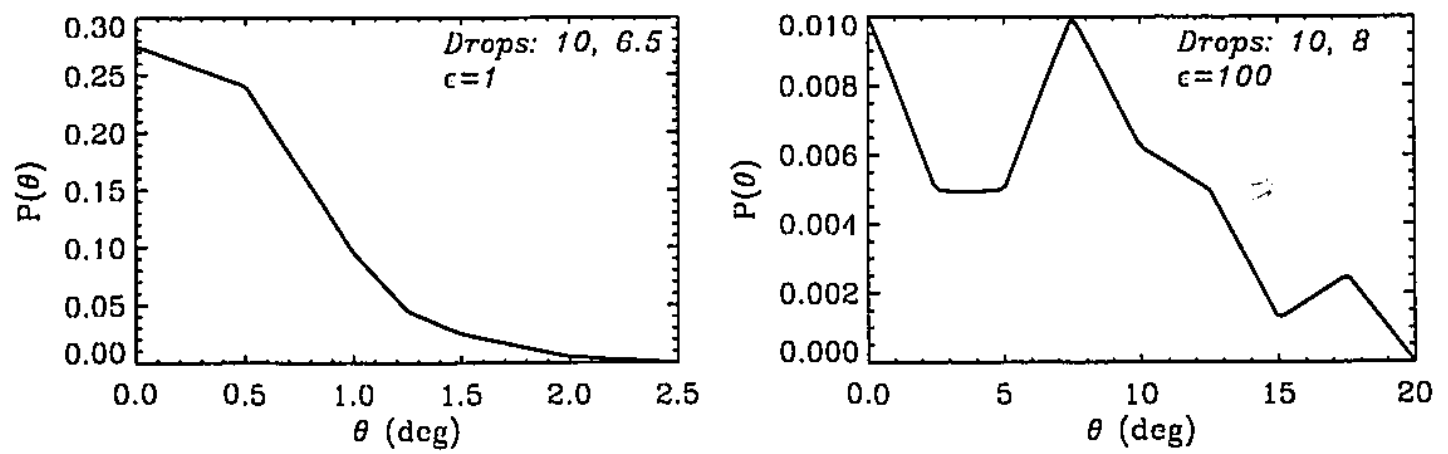

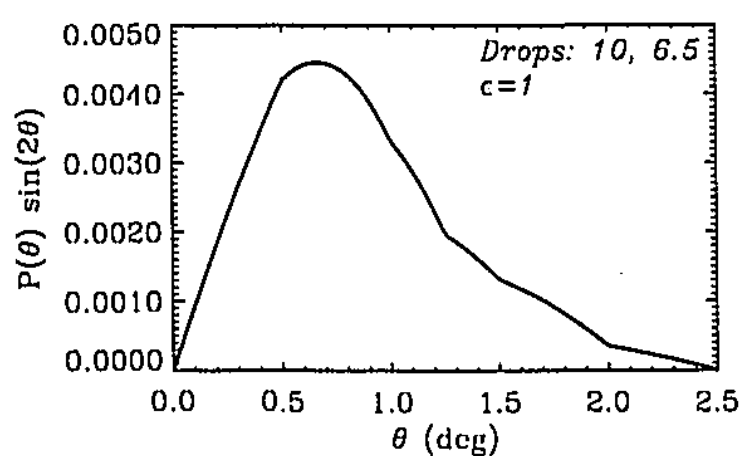

a) Weak turbulence case

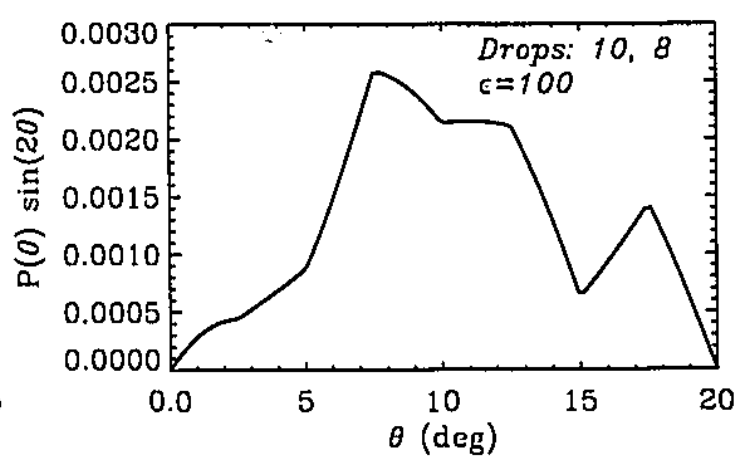

b) Strong turbulence case

Fig. 8.1 Probability $P(\theta)$ and $P(\theta) \sin 2 \theta$ for the typical case of: a) a 'weak turbulence' case $\left(r_{1}=10 \mu \mathrm{m}, r_{2}=6.5 \mu \mathrm{m}, \epsilon=1 \mathrm{~cm}^{2} \mathrm{sec}^{-3}\right)$, and b) a 'strong turbulence' case $\left(r_{1}=10 \mu \mathrm{m}, r_{2}=8 \mu \mathrm{m}, \epsilon=100 \mathrm{~cm}^{2} \mathrm{sec}^{-3}\right)$. 
In order to determine the total number of experiments we made an extensive use of error analysis. (Error analysis is described in Section 8.4.) Our goal was to obtain errors within the limit of $15 \%$. For many parameters $\left(r_{1}, r_{2}\right.$, and $\left.\epsilon\right)$, however, much better accuracies were obtained. Unfortunately, for some other parameters to meet the above criterion was very difficult, and, sometimes, impossible. These were scenarios with very few collisions, and, consequently, very low probabilities $P(\theta)$ which required literally thousands of experiments to be performed.

\subsection{Calculation of the Average 'in' Component of the Radial Velocity}

In Chapter 6, we introduced a modification to the flux method aimed at including the variation of the radial 'in' component of the drop relative velocity. For large drop radius ratios and stronger turbulence, the average value of the radial 'in' component of the drop relative velocity, $w_{r}^{\text {in }}$ is not equal to $w_{\iota} \cos \theta$. We expected that difference to be rather small, yet, it seemed to be necessary to investigate the issue more thoroughly. The following numerical experiment was designed to calculate $w_{r}^{i n}$. The geometry of the experiment was identical to that used in the calculation of trajectories. The collector drop was placed at the origin, and the collected drop at the position $\left(R+\Delta R, \theta_{0}, \phi=0\right) ; \Delta R \ll R$. Then, the ordinary trajectory modelling was carried out up to the point when the distance between drops was $R$. Subsequently, $w_{r}^{i n}$ and the azimuthal angle $\theta$ were recorded, and later averaged. Starting the cxperiments at the surface of the sphere of radius $R+\Delta R$ was aimed toward relaxing the initial conditions imposed on the drops. The averaged value of $\theta$ was very close to the initial value of $\theta_{0}$. In Fig. 8.2, there are shown two examples of the curves $w_{r}^{i n}$ vs. $\theta$ for the laminar and turbulent cases for two sets of parameters: a) $r_{1}=10 \mu \mathrm{m}$, 
$r_{2}=9.5 \mu \mathrm{m}, \epsilon=100 \mathrm{~cm}^{2} \mathrm{sec}^{-3}$, and b) $r_{1}=10 \mu \mathrm{m}, r_{2}=5 \mu \mathrm{m}, \epsilon=1 \mathrm{~cm}^{2} \mathrm{sec}^{-3}$. The experiments demonstrate that the effect of turbulence on $w_{r}^{i n}$ is very small, in fact negligible in most of the considered sets of parameters.

In Chapter 6, we also introduced the weighting function, $f(\theta)$ which represents the fraction of drops which have a positive $w_{r}^{i n}$. For $\epsilon=100 \mathrm{~cm}^{2} \mathrm{sec}^{-3}$, this fraction was found to be equal to unity for all angles $\theta<90^{\circ}$ up to the radii ratio of the order of 0.9 . For higher radii ratio, $f(\theta)$ decreases being, as expected, of the order of 0.5 for equal drops. In our experiment aimed toward obtaining collision efficiencies for unequal drops, we found that $f^{\prime}(\theta)=1$ for all angles $\theta$ with nonzero probability of collision.

a)

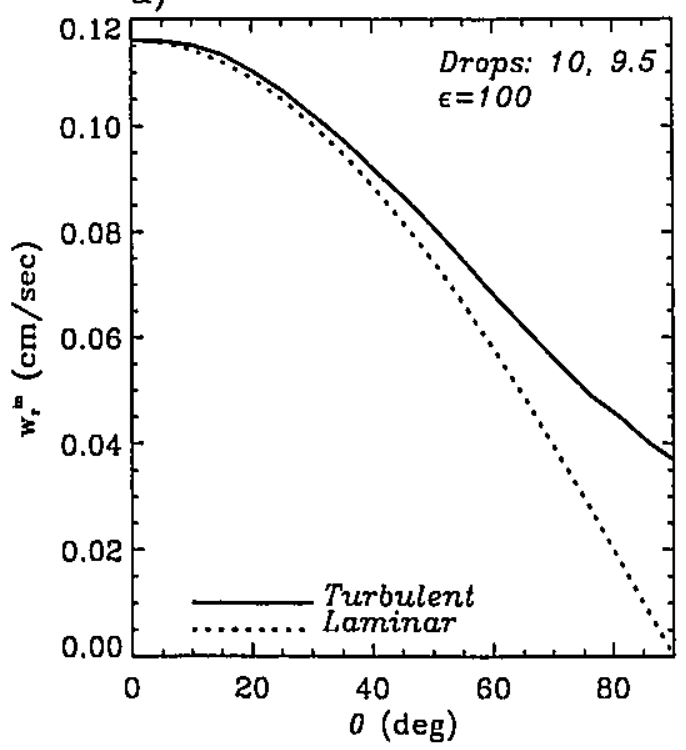

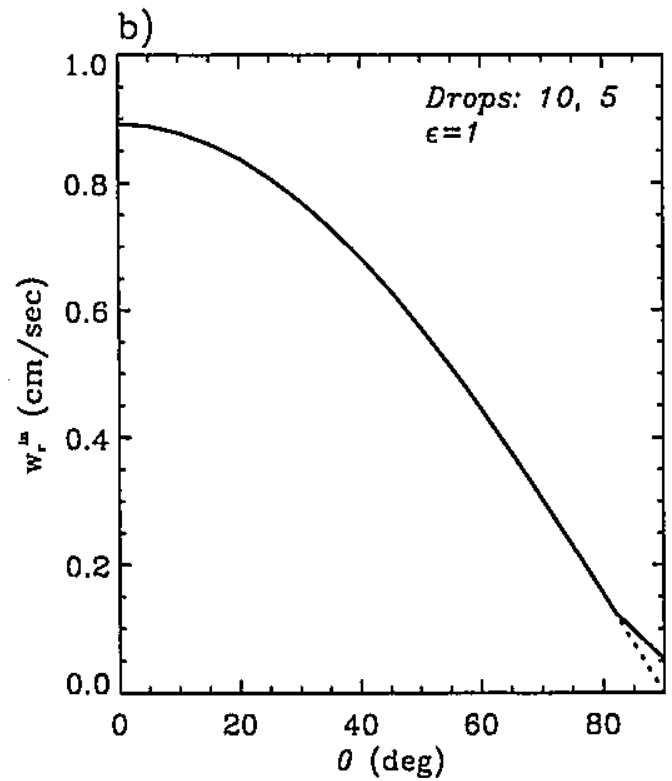

Fig. 8.2 Comparison of the average radial 'in' component of the drop relative velocity for turbulent and laminar cases: a) $r_{1}=10 \mu \mathrm{m}, r_{2}=9.5 \mu \mathrm{m}, \epsilon=100 \mathrm{~cm}^{2}$ $\mathrm{sec}^{-3}$, b) $r_{1}=10 \mu \mathrm{m}, r_{2}=5 \mu \mathrm{m}, \epsilon=1 \mathrm{~cm}^{2} \mathrm{sec}^{-3}$. if 


\subsection{Tests and Justifications}

\subsubsection{The Dependence of the Collision Efficiency on the Initial Distance between Drops}

In this section, we attempt to answer two questions: 1 . whether the initial distance between drops, $R$ of the order of 10 or 20 radii of the collector drop is sufficient from the point of view of the hydrodynamic interactions for calculation of the collision efficiencies? 2. whether or not the collision efficiencies depend on $R$ in general? In order to answer the first question, let us once again take a look at the laminar still-air case. The relative displacements of drops are more dramatic for the nonzero initial horizontal offsets of drops as opposed to the zero horizontal offset. In the former case, the strongest hydrodynamic forces along the line of centres have horizontal components, and in the latter case, do not. We will direct our attention to two cases of nonzero horizontal offsets: the case of grazing trajectory, and the case of the horizontal offset equal to the sum of radii. The idea is to compare the relative horizontal displacements due to the hydrodynamic forces with those due to turbulence. We simplify the problem by assuming that drop inertia is neglected, and that drop movement in the horizontal plane is the same as that of fluid parcels. Also, we assume that the relative horizontal displacement of the drops is composed of the turbulent and hydrodynamic parts as follows

$$
\Delta \xi=\Delta \xi_{T}-\Delta \xi_{H},
$$

where $\Delta \xi$ is the total horizontal displacement of drops during the time period in which the vertical distance between drops changes from $\zeta$ to $\zeta-\Delta \zeta$, and $\Delta \xi_{T}$ and $\Delta \xi_{H}$ are the corresponding displacements due to turbulence and hydrodynamic interactions. Further, for the displacement due to turbulence, we can write

$$
\Delta \xi_{T}=\frac{\partial u_{x}}{\partial z} \zeta \Delta t=\frac{\partial u_{x}}{\partial z} \zeta \frac{\Delta \zeta}{w_{t}} .
$$


It is convenient to use the ratio

$$
\frac{\Delta \xi}{\Delta \zeta}=\frac{\partial u_{x}}{\partial z} \frac{\zeta}{w_{t}}-\underbrace{\frac{\Delta \xi_{H}}{\Delta \zeta}}_{h(\zeta)}
$$

$h(\zeta)$ represents the rate of drop horizontal displacement per unit change in vertical separation due to hydrodynamic interactions. Because $u_{x}$ is the instantaneous turbulent velocity, the r.m.s. of $\Delta \xi / \Delta \zeta$ gives the magnitude of the displacement. Let us calculate the average

$$
\overline{\left(\frac{\Delta \xi}{\Delta \zeta}\right)^{2}}=\overline{\left(\frac{\partial u_{x}}{\partial z}\right)^{2}}\left(\frac{\zeta}{w_{t}}\right)^{2}-2 \underbrace{\overline{\frac{\partial u_{x}}{\partial z}}}_{=0} \frac{\zeta}{w_{t}} h(\zeta)+h(\zeta)^{2}=\overline{\left(\frac{\partial u_{x}}{\partial z}\right)^{2}}\left(\frac{\zeta}{w_{t}}\right)^{2}+h(\zeta)^{2} .
$$

The r.m.s. value of the velocity derivative can be approximated by

$$
\sqrt{\left(\frac{\partial u_{x}}{\partial z}\right)^{2}}=\underbrace{\sqrt{\frac{2 \epsilon}{15 \nu}}}_{L}
$$

[Taylor, 1935]. Finally,

$$
\sqrt{\left(\frac{\Delta \xi}{\Delta \zeta}\right)^{2}}=\sqrt{\left(\frac{L \zeta}{w_{t}}\right)^{2}+h(\zeta)^{2}}
$$

where $(L \zeta) / w_{t}$ represents the r.m.s. rate of horizontal displacement per unit change in vertical separation due to turbulence alone. Therefore, if the rate of displacement due to hydrodynamic forces, $h(\zeta)$, is much smaller than the r.m.s. rate of displacement due to turbulence, the hydrodynamic interactions can be neglected.

Let us now be more precise about what 'much smaller' really means. In order to quantitatively determine the distance between drops beyond which the hydrodynamic interactions can be neglected, we calculate the error of the rate of horizontal displacement per unit change in vertical separation if the hydrodynamic forces are indeed neglected

$$
\frac{\left|\sqrt{\left(\frac{\Delta \xi}{\Delta \zeta}\right)^{2}}-\frac{L \zeta}{w_{t}}\right|}{\sqrt{\left(\frac{\Delta \xi}{\Delta \zeta}\right)^{2}}}=\left|1-\frac{1}{\sqrt{1+\delta(\zeta)^{2}}}\right| \approx \delta^{2},
$$


where

$$
\delta(\zeta)=\frac{h(\zeta)}{(L \zeta) / w_{t}}
$$

Note that for the case of a constant shear, the analogous error is equal to

$$
\left|\frac{\delta(\zeta)}{1-\delta(\zeta)}\right| \approx \delta
$$

Therefore, if $\delta$ is small, the hydrodynamic interactions can be neglected more convincingly for the case of turbulent flow than for the case of linear shear. Further, if we assume the error tolerance $<1 \%, \delta<0.1$.

In Fig. 8.3, there are presented the r.m.s values of the rate of horizontal displacements per unit change in vertical separation, $(L \zeta) / w_{t}$ for different values of the rate of energy dissipation $\left(0.01-100 \mathrm{~cm}^{2} \mathrm{sec}^{-3}\right)$, and the rates of horizontal displacements per unit change in vertical separation due to hydrodynamic interaction, $h(\zeta)$ for two laminar trajectories, the grazing trajectory, and the trajectory obtained for the initial horizontal offset, $y$ equal to the sum of radii. In particular, we chose to present the above quantities for the case of large radius ratio (drop radii 10 and 9 $\mu \mathrm{m}$ ), and for small radius ratio (drop radii 15 and $5 \mu \mathrm{m}$ ). From the information displayed in Fig. 8.3, we conclude that for all the considered cases, a relative separation of the order of 20 radii of the collector drop is sufficient to assure errors $<1 \%$ for $\epsilon \geq 1 \mathrm{~cm}^{2} \mathrm{sec}^{-3}$. Also, for radius ratios greater than 0.9 , that distance can be diminished to 10 radii for stronger turbulence, $\epsilon \geq 100 \mathrm{~cm}^{2} \mathrm{sec}^{-3}$. We used the trajectory $y=r_{1}+r_{2}$ to estimate $\delta$. Because the displacements due to the hydrodynamic forces for $y=r_{1}+r_{2}$ are the largest, and they decrease with increasing $\zeta$, our estimates are rather conservative. 

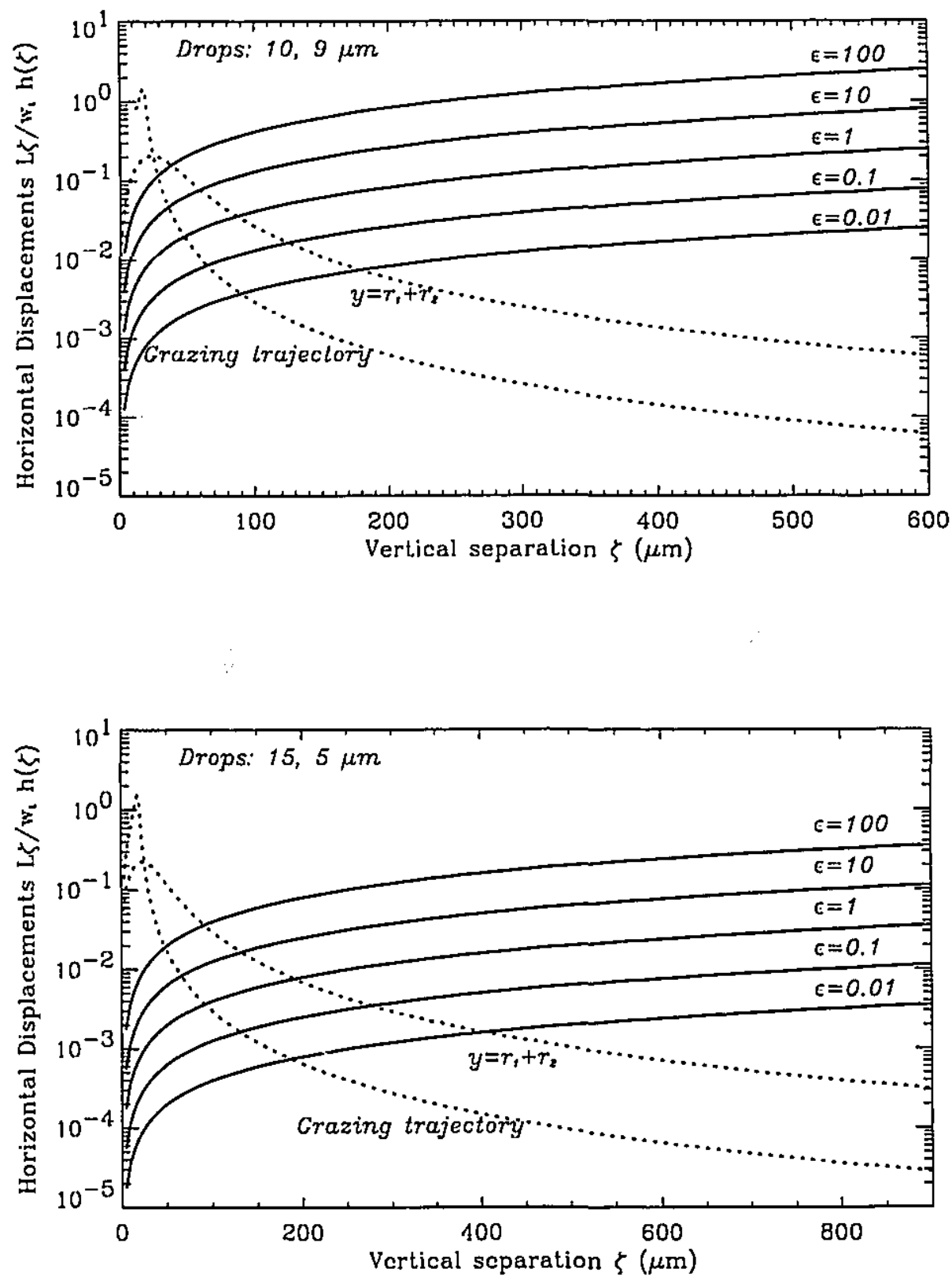

Fig. 8.3 Comparison of the r.m.s. rate of horizontal displacements per unit change in vertical separation due to turbulence and the rate of horizontal displacements per unit change in vertical separation due to hydrodynamic forces for two pairs of drops: 10 and $9 \mu \mathrm{m}$, and 15 and $5 \mu \mathrm{m}$. Solid lines represent the displacements due to turbulence for the energy dissipation rates from 0.01 to $100 \mathrm{~cm}^{2} \mathrm{sec}^{-3}$. Dashed lines represent the displacements due to the hydrodynamic forces for laminar still-air settling. 
In Chapter 6, we proved that the collision kernels (and the collision efficiencies) calculated using the flux method do not depend on the initial distance between drops, $R$. We chose two sets of parameters $\left(r_{1}, r_{2}\right.$, and $\left.\epsilon\right)$ in order to practically assess the dependence on $R$. The results of these tests are presented in Table 8.1. We purposely choose cases with the radius ratios equal to 0.8 in order to have a stronger effect of turbulence-not so strong, however, as to make the calculation completely inconclusive due to statistical errors. In Table 8.1, we include also the statistical errors of the calculations. The collision efficiencies obtained for two different values of $R$ are within the error range. Thus, we conclude that our numerical calculation does not contradict the assumption that the collision efficiencies are independent of $R$.

TABLE 8.1

The Dependence of the Collision Efficiency on the Initial Distance between Drops

\begin{tabular}{cccccc}
\hline $\begin{array}{c}\text { Radius } r_{1} \\
(\mu \mathrm{m})\end{array}$ & $\begin{array}{c}\text { Radius } r_{2} \\
(\mu \mathrm{m})\end{array}$ & $\begin{array}{c}\text { Radius } R \\
(\mu \mathrm{m})\end{array}$ & $\begin{array}{c}\epsilon \\
\left(\mathrm{cm}^{2} \mathrm{sec}^{-3}\right)\end{array}$ & Collision Efliciency & $\begin{array}{c}\text { Error } \\
\%\end{array}$ \\
\hline 15 & 12 & 150 & 100 & 0.017 & 10 \\
15 & 12 & 300 & 100 & 0.015 & 10 \\
10 & 9 & 100 & 10 & 0.016 & 6 \\
10 & 9 & 200 & 10 & 0.017 & 19 \\
\hline
\end{tabular}

\subsubsection{The Dependence of the Collision Efficiency on the Trun- cation of the Turbulent Field}

A series of experiments was designed in order to prove that the truncation performed on the basis of scale analysis does not affect the calculated values of collision 
efficiencies. The experiments resulted in a full success. In Table 8.2, we present the results for two pairs of drops, with weak and strong turbulence. The quantity termed in the table 'Truncation Factor' represents the truncation factor of the length and time scales of turbulence with comparison to the length and time scale of the drop interactions. In particular, the truncation factor of 50 means that all the scales (space and time) greater than 50 times the respective length and time scale of interaction are truncated. (Note that the truncation factor of 25 represents a higher truncation than that of 50.) Clearly, for the cases with higner truncations, the values of the collision kernels are reproduced very accurately in comparison to the less truncated cases. In other words, the modes which were rejected did not have any influence on the collisional problem.

TABLE 8.2

The Dependence of the Collision Efficiency on Turbulence Truncation

\begin{tabular}{ccccccc}
\hline $\begin{array}{c}\text { Radius } r_{1} \\
(\mu \mathrm{m})\end{array}$ & $\begin{array}{c}\text { Radius } r_{2} \\
(\mu \mathrm{m})\end{array}$ & $\begin{array}{c}\text { Radius } R \\
(\mu \mathrm{m})\end{array}$ & $\begin{array}{c}\epsilon \\
\left(\mathrm{cm}^{2} \mathrm{sec}^{-3}\right)\end{array}$ & $\begin{array}{c}\text { Truncation } \\
\text { Factor }\end{array}$ & $\begin{array}{c}\text { Collision } \\
\text { Efficiency }\end{array}$ & $\begin{array}{c}\text { Error } \\
\%\end{array}$ \\
\hline 20 & 6 & 400 & 1 & 200 & 0.0126 & 3 \\
20 & 6 & 400 & 1 & 100 & 0.0127 & 2 \\
10 & 8 & 200 & 100 & 50 & 0.0139 & 20 \\
10 & 8 & 200 & 100 & 25 & 0.0144 & 19 \\
\hline
\end{tabular}

\subsubsection{The no-Effect Limit of the Rate of Energy Dissipation}

The questions which we were frequently asked during the test stage of the model were whether we can reproduce laminar collision efficiencies by calculations with very weak turbulence, and how weak must the turbulent field be to do so. Because for very weak turbulence, the values of $R$ equal to 20 radii of the collector drop is not sufficient 
to neglect the hydrodynamic interaction on the sphere of radius $R$, the comparisons were made with laminar values obtained for the same geometry (i.e. $R=20 r_{1}$ ). In Fig. 8.4, there are presented the collision efficiency curves for 10 and $15 \mu \mathrm{m}$ collector drops for the laminar case, and for the turbulent case with $\epsilon=0.01 \mathrm{~cm}^{2} \mathrm{sec}^{-3}$. The agreement is very good. Using the results of this experiment we were able not only to reassure ourselves that the model is robust but also to find the lower limit of the rate of energy dissipation which might have an effect, on collision rates.

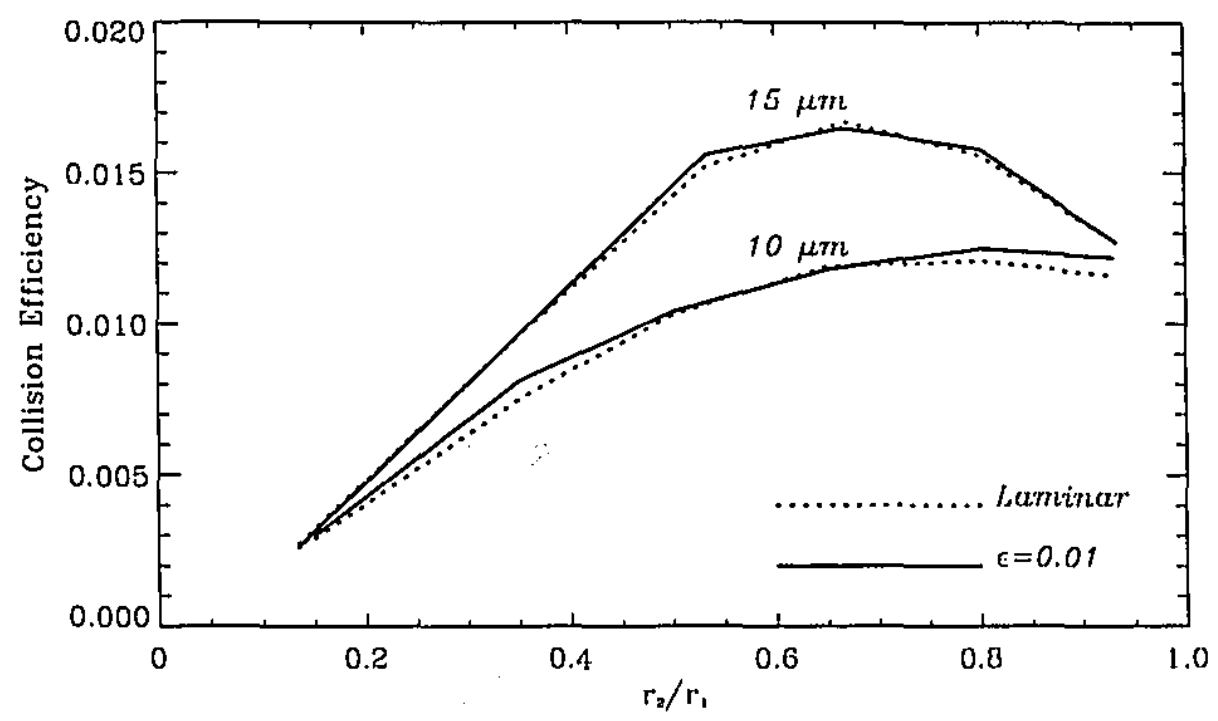

Fig. 8.4 Comparison of the laminar coilision efficiencies with the turbulent collision efficiencies for the rate of energy dissipation equal to $0.01 \mathrm{~cm}^{2} \mathrm{sec}^{-3}$.

\subsection{Error Analysis}

Let us recall the expression for the collision efficiency

$$
E=\frac{R^{2}}{\left(r_{1}+r_{2}\right)^{2}} \int_{0}^{o_{\max }} P(\theta ; R) \sin 2 \theta d \theta
$$


The accuracy of the above expression depends on the accuracy with which we calculate the probability of collision, $P(\theta ; R)$. The latter, being a product of modelling numerous drop trajectories, has two sources of errors: one associated with the accuracy of the trajectory calculation, and the other associated with the statistical variance. We discussed the accuracy of calculations of drop trajectories in Chapter 5-the numerical errors are negligible. In Section 8.3.1, we also discussed the errors due to neglecting the effect of hydrodynamic interactions for the drop separations greater than 20 radii of the collector drop. We showed that the maximum errors for a single trajectory in the most unfavourable situations were very small $(\leq 1 \%)$. Therefore, the errors due to neglecting the hydrodynamic interactions at larger drop separations are small in comparison to the statistical errors which are usually greater than $5 \%$.

Let us present the method with which to obtain the statistical variance of the collision efficiency. In order to do so, we represent the collision efficiency in the following approximate form

$$
E=\frac{R^{2}}{\left(r_{1}+r_{2}\right)^{2}} \sum_{i=1}^{n-1} \frac{P\left(\theta_{i}\right)+P\left(\theta_{i+1}\right)}{2} \int_{\theta_{i}}^{\theta_{i+1}} \sin 2 \theta d \theta=\frac{R^{2}}{\left(r_{1}+r_{2}\right)^{2}} \sum_{i=1}^{n-1} I_{i},
$$

where $n$ is the number of the values of $\theta$ used to estimate the collision efficiency. (Fig. 8.5 should be helpful to understand the notation.) The variance of the collision efficiency is then

$$
\operatorname{var}(E)=\left[\frac{R^{2}}{\left(r_{1}+r_{2}\right)^{2}}\right]^{2} \sum_{i=1}^{n-1} \operatorname{var}\left(I_{i}\right)
$$

Further, we can calculate

$$
\begin{gathered}
\operatorname{var}\left(I_{i}\right)=\frac{1}{4}\left(\int_{\theta_{i}}^{\theta_{i+1}} \sin 2 \theta d \theta\right)^{2}\left[\operatorname{var}\left(P_{i}\right)+\operatorname{var}\left(P_{i+1}\right)\right]= \\
\frac{1}{16}\left(\cos 2 \theta_{i}-\cos 2 \theta_{i+1}\right)^{2}\left[\operatorname{var}\left(P_{i}\right)+\operatorname{var}\left(P_{i+1}\right)\right]
\end{gathered}
$$

where $P_{i}=P\left(\theta_{i}\right):$ In the next step we will show how to estimate $\operatorname{var}\left(P_{i}\right) . \quad P_{i}$ is calculated as a ratio of the number of collisions, $N_{\text {coll }}^{i}$, to the total number of 
experiments, $N_{e x p}^{i}$. Let the occurrence of a collision be called an event. Suppose that we perform an experiment which results in the indicator of an event taking either the value of 1 (collision) or the value of 0 (no collision). Let us also assume that the probability of the occurrence of a collision is $p$ and the probability of nocollision is $1-p$. The variance of obtaining $N_{\text {coll }}^{i}$ collisions in $N_{e x p}^{i}$ experiments is then $N_{e x p}^{i} p(1-p)$. Thus the variance of the calculated value $P_{i}$ is

$$
\operatorname{var}\left(P_{i}\right)=\frac{p(1-p)}{N_{e x p}^{i}} .
$$

Finally, if we estimate the value of $p$ by $P_{i}$ we have

$$
\operatorname{var}\left(P_{i}\right)=\frac{P_{i}\left(1-P_{i}\right)}{N_{e x p}^{i}}
$$

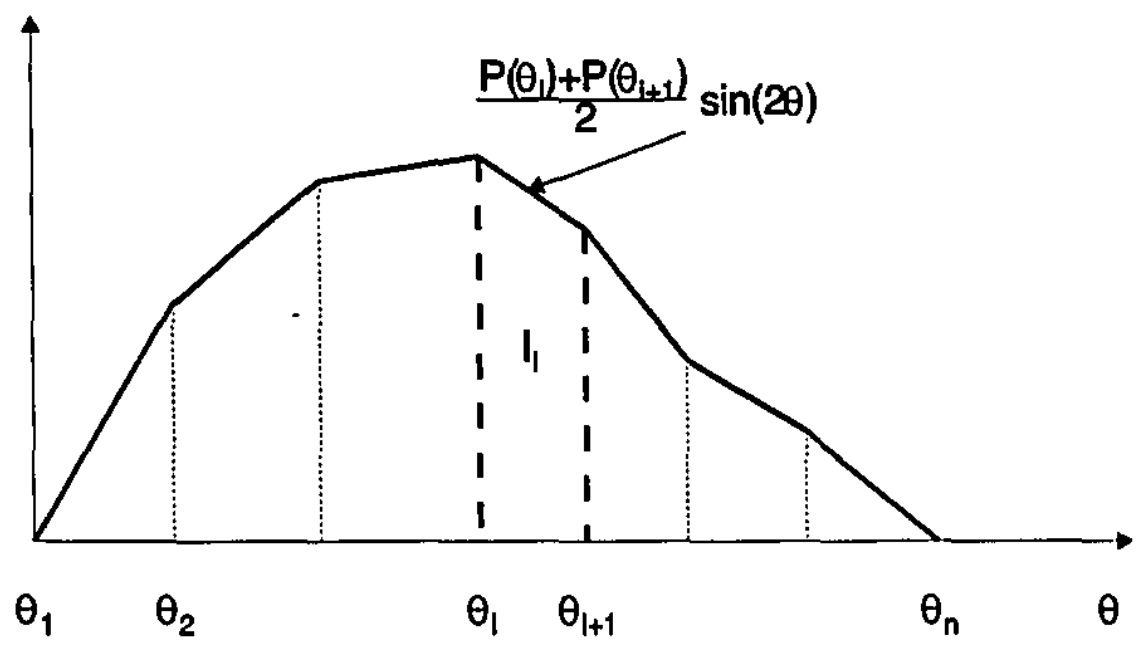

Fig. 8.5 Schematic sketch for error calculation.

Naturally, the standard deviation of a quantity represents the error. Therefore, the final expression for the error of the collision efficiency is

$$
\sigma(E)=\frac{R^{2}}{\left(r_{1}+r_{2}\right)^{2}} \frac{1}{4}\left\{\sum_{i=1}^{n-1}\left(\cos 2 \theta_{i}-\cos 2 \theta_{i+1}\right)^{2}\left[\frac{P_{i}\left(1-P_{i}\right)}{N_{\text {exp }}^{i}}+\frac{P_{i+1}\left(1-P_{i+1}\right)}{N_{e x p}^{i+1}}\right]\right\}^{\frac{1}{2}} .
$$


It follows from the above equation that for $N_{\text {coll }}^{i} \rightarrow N_{e x p}^{i}, \sigma \rightarrow 0$, and for very small $N_{\text {coll }}^{i}, \sigma \propto 1 / \sqrt{N_{e x p}^{i}}$. Thus, in the latter case $\sigma$ decreases with the number of experiments. 


\section{9 \\ Discussion of the Results}

Here, there are presented the collision efficiencies for different radii and different strengths of turbulence. The results are also discussed in terms of a scale analysis. Finally, the extension of the model for equal drops is outlined.

\subsection{Presentation of the Basic Results}

We systematically examined three different collector drops, radii 10, 15, and 20 $\mu \mathrm{m}$. For each collector drop, there were chosen six to seven small drops for which we obtained the collision efficiencies. The selected rates of energy dissipation were 1,10 , and $100 \mathrm{~cm}^{2} \mathrm{sec}^{-3}$. In Table 9.1, there are summarized the collision efficiencies for different values of the drop radii and the rate of energy dissipation. The parameters required by the model (as described in Chapter 8) which were applied in the calculation, and the estimated statistical errors are also included. In Figs. 9.1, and 9.2-9.4, data from Table 9.1 are juxtaposed with the laminar collision efficiencies. The four panels of Fig. 9.1 feature the respective collision efficiencies for three cases of turbulent calculations, and the laminar calculation. They illustrate different characters of the collision efficiency curves for different strengths of turbulence (and no-turbulence). Figs. 9.2-9.4 are designed to compare the values of the collision efficiencies for the laminar and turbulent cases for the same collector drop. 
TABLE 9.1

Turbulent Collision Efficiencies

for Different Radii and Different Rates of Energy Dissipation

\begin{tabular}{|c|c|c|c|c|c|c|c|}
\hline $\begin{array}{c}\text { Racdius } r_{1} \\
\qquad(\mu \mathrm{m})\end{array}$ & $\begin{array}{c}\text { Radius } r_{2} \\
(\mu \mathrm{m})\end{array}$ & $\left(\operatorname{cm}^{2} \sec ^{-3}\right)$ & $\begin{array}{l}\text { Radius } R \\
\quad(\mu \mathrm{m})\end{array}$ & $\begin{array}{l}\text { Truncation } \\
\text { Factor }\end{array}$ & $\begin{array}{l}\text { Collision } \\
\text { Efficiency }\end{array}$ & $\begin{array}{c}\text { Error } \\
(\%)\end{array}$ & $\begin{array}{c}\text { Relative } \\
\text { Change (\%) } \dagger\end{array}$ \\
\hline 10 & 1.5 & 1 & 200 & 100 & 0.0040 & 7 & +53 \\
\hline 10 & 3.5 & 1 & 200 & 100 & 0.0092 & 9 & +33 \\
\hline 10 & 5.0 & 1 & 200 & 100 & 0.0114 & 11 & +22 \\
\hline 10 & 6.5 & 1 & 200 & 50 & 0.0124 & 6 & +15 \\
\hline 10 & 8.0 & 1 & 200 & 50 & 0.0122 & 10 & +12 \\
\hline 10 & 9.3 & 1 & 200 & 50 & 0.0124 & 14 & -36 \\
\hline 10 & 1.5 & 10 & 200 & 50 & 0.0036 & 9 & +32 \\
\hline 10 & 3.5 & 10 & 200 & 50 & 0.0107 & 15 & +55 \\
\hline 10 & 5.0 & 10 & 200 & 50 & 0.0127 & 7 & +46 \\
\hline 10 & 6.5 & 10 & 200 & 50 & 0.0135 & 15 & +25 \\
\hline 10 & 8.0 & 10 & 100 & 50 & 0.0160 & 6 & +47 \\
\hline 10 & 9.3 & 10 & 100 & 50 & 0.0101 & 14 & -2 \\
\hline 10 & 1.5 & 100 & 100 & 50 & 0.0033 & 21 & +24 \\
\hline 10 & 3.5 & 100 & 100 & 50 & 0.0088 & 11 & +28 \\
\hline 10 & 5.0 & 100 & 100 & 50 & 0.0101 & 10 & +8 \\
\hline 10 & 6.5 & 100 & 100 & 50 & 0.0110 & 21 & +2 \\
\hline 10 & 8.0 & 100 & 100 & 50 & 0.0144 & 19 & +32 \\
\hline 10 & 9.3 & 100 & 100 & 50 & 0.0137 & 11 & +29 \\
\hline 15 & 2.0 & 1 & 300 & 200 & 0.0024 & 12 & -2 \\
\hline 15 & 5.0 & 1 & 300 & 50 & 0.0100 & 6 & +20 \\
\hline 15 & 8.0 & 1 & 300 & 50 & 0.0156 & 3 & +12 \\
\hline 15 & 10.0 & 1 & 300 & 50 & 0.0175 & 6 & +15 \\
\hline 15 & 12.0 & 1 & 300 & 50 & 0.0171 & 5 & +22 \\
\hline 15 & 14.0 & 1 & 300 & 50 & 0.0124 & 6 & -1 \\
\hline 15 & 2.0 & 10 & 300 & 50 & 0.0035 & 12 & +43 \\
\hline 15 & 5.0 & 10 & 300 & 50 & 0.0112 & 7 & +34 \\
\hline 15 & 8.0 & 10 & 300 & 50 & 0.0156 & 3 & +13 \\
\hline 15 & 10.0 & 10 & 300 & 50 & 0.0155 & 8 & +1 \\
\hline 15 & 12.0 & 10 & 300 & 50 & 0.0178 & 8 & +26 \\
\hline 15 & 14.0 & 10 & 300 & 50 & 0.0100 & 8 & -12 \\
\hline 15 & 2.0 & 100 & 150 & 50 & 0.0026 & 15 & +7 \\
\hline 15 & 5.0 & 100 & 300 & 50 & 0.0052 & 9 & -38 \\
\hline 15 & 8.0 & 100 & 300 & 50 & 0.0136 & 8 & -2 \\
\hline 15 & 10.0 & 100 & 300 & 50 & 0.0129 & 18 & -15 \\
\hline 15 & 12.0 & 100 & 150 & 50 & 0.0171 & 9 & +22 \\
\hline 15 & 14.0 & 100 & 150 & 50 & 0.0138 & 11 & +21 \\
\hline
\end{tabular}

† This column refers to the relative change of the turbulent collision efficiency with respect to the laminar collision efficiency. 
TABLE 9.1 Cont.

Turbulent Collision Efficiencies

for Different Radii and Different Rates of Energy Dissipation

\begin{tabular}{cccccccc}
\hline $\begin{array}{c}\text { Radius } r_{1} \\
(\mu \mathrm{m})\end{array}$ & $\begin{array}{c}\text { Radius } r_{2} \\
(\mu \mathrm{m})\end{array}$ & $\begin{array}{c}\epsilon \\
\left(\mathrm{cm}^{2} \mathrm{sec}^{-3}\right)\end{array}$ & $\begin{array}{c}\text { Radius } R \\
(\mu \mathrm{m})\end{array}$ & $\begin{array}{c}\text { Truncation } \\
\text { Factor }\end{array}$ & $\begin{array}{c}\text { Collision } \\
\text { Efficiency }\end{array}$ & $\begin{array}{c}\text { Error } \\
(\%)\end{array}$ & $\begin{array}{c}\text { Relutive } \\
\text { Chumge }(\%)\end{array}$ \\
\hline 20 & 3.0 & 1 & 400 & 200 & 0.0037 & 8 & +8 \\
20 & 6.0 & 1 & 400 & 200 & 0.0126 & 3 & +10 \\
20 & 9.0 & 1 & 400 & 200 & 0.0278 & 4 & +17 \\
20 & 12.0 & 1 & 400 & 100 & 0.0387 & 2 & +20 \\
20 & 14.0 & 1 & 400 & 100 & 0.0391 & 6 & +27 \\
20 & 16.0 & 1 & 400 & 50 & 0.0284 & 2 & +18 \\
20 & 19.0 & 1 & 400 & 50 & 0.0160 & 8 & +26 \\
\hline 20 & 3.0 & 10 & 400 & 50 & 0.0046 & 14 & +36 \\
20 & 6.0 & 10 & 400 & 50 & 0.0144 & 2 & +26 \\
20 & 9.0 & 10 & 400 & 50 & 0.0277 & 7 & +16 \\
20 & 12.0 & 10 & 400 & 50 & 0.0323 & 3 & -0 \\
20 & 14.0 & 10 & 400 & 50 & 0.0360 & 6 & +19 \\
20 & 16.0 & 10 & 400 & 50 & 0.0262 & 4 & +9 \\
20 & 19.0 & 10 & 400 & 50 & 0.0111 & 14 & -13 \\
\hline 20 & 3.0 & 100 & 400 & 50 & 0.0003 & 14 & -91 \\
20 & 6.0 & 100 & 400 & 50 & 0.0164 & 7 & +44 \\
20 & 9.0 & 100 & 400 & 50 & 0.0331 & 13 & +39 \\
20 & 12.0 & 100 & 400 & 50 & 0.0287 & 7 & -11 \\
20 & 14.0 & 100 & 400 & 50 & 0.0349 & 11 & +13 \\
20 & 16.0 & 100 & 400 & 50 & 0.0389 & 6 & +61 \\
20 & 19.0 & 100 & 200 & 50 & 0.0140 & 11 & +10 \\
\hline
\end{tabular}

In Chapters 6 and 8 , we introduced corrections to the collision kernels and collision efficiencies related to the discrepancy between the laminar and turbulent radial 'in' component of the relative velocity. These corrections turned out to be insignificant for the majority of considered cases. In Table 9.1, and Figs. 9.1-9.4, the corrections are included only for ratios of drop radii greater than 0.9 , and $\epsilon=100$ $\mathrm{cm}^{2} \mathrm{sec}^{-3}$. For such values, the corrections are of the order of $1-7 \%$ while for smaller ratios of drop radii and lower rates of energy dissipation, the corrections are less than $1 \%$. 

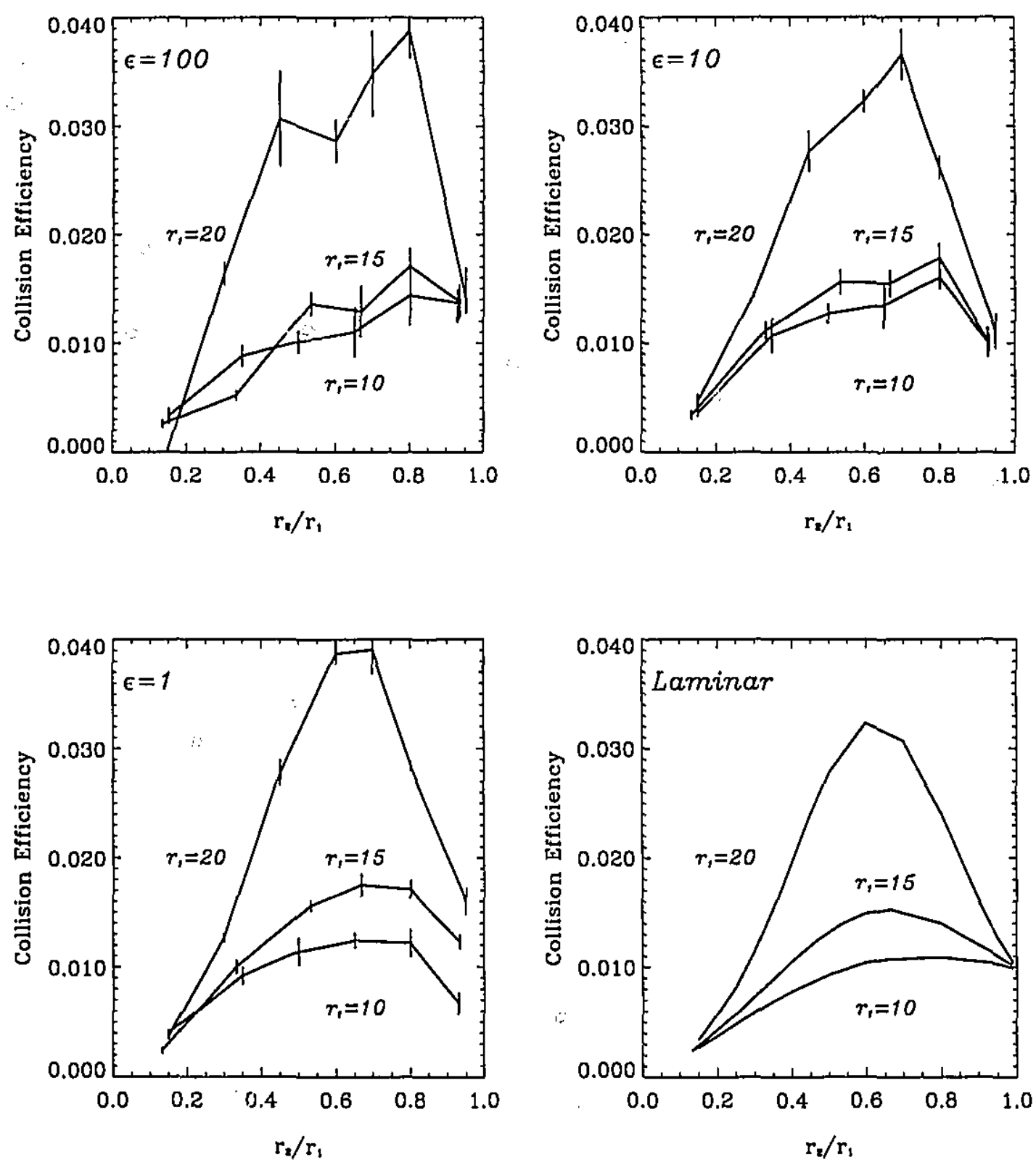

Fig. 9.1 Collision efficiencies for turbulent flows with different rates of energy dissipation $\left(\epsilon=100,10,1 \mathrm{~cm}^{2} \mathrm{sec}^{-3}\right)$, and for laminar flow. 
Returning to the interpretation of the results, from Fig. 9.1, it is clear that the behaviour of the turbulent collision efficiency curves reveals two regimes, one for 'weak' turbulence $\left(\epsilon \leq 1 \mathrm{~cm}^{2} \mathrm{sec}^{-3}\right)$, and another for 'strong' turbulence $(\epsilon \geq 10$ $\left.\mathrm{cm}^{2} \mathrm{sec}^{-3}\right)$. Both regimes are characterized by a moderate increase of the collision efficiencies with respect to laminar flow. However, the domains of radius ratios where the increases take place are different. The 'strong' turbulence regime is responsible for shifts of the peaks of the collision efficiencies toward higher radius ratios in comparison to the laminar collision efficiencies. Also, in this regime, one can observe peculiar local minima of the collision efficiencies which occur for ratios of drop radii equal roughly to 0.7. The 'weak' turbulence regime, on the other hand, seems to be more consistent with the laminar case. The character of the collision efficiency curves resembles that: of the laminar collision efficiency curves except for ratios of drop radii greater than 0.9. For large radius ratios, the collision efficiency values for different sized collector drops are quite different whereas, according to the applied hydrodynamic model, in the laminar case they tend to the same value as the radius ratio approaches 1.

Switching our attention to Figs. 9.2-9.4, we can determine the strength of turbulence which causes the highest increases of the collision efficiencies for different ratios of drop radii. (Table 9.1 is helpful as well.) For $10 \mu \mathrm{m}$ collector drops, the effecti seems to be the strongest $(\approx 50 \%$ increase) for the rate of energy dissipation equal to $10 \mathrm{~cm}^{2} \mathrm{sec}^{-3}$, except for the radius ratio equal to 0.93 . The collision efficiency for $\epsilon=1 \mathrm{~cm}^{2} \mathrm{sec}^{-3}$ increases in the same manner as for $\varepsilon=10 \mathrm{~cm}^{2} \mathrm{sec}^{-3}$ but in a more moderate way. For $\epsilon=100 \mathrm{~cm}^{2} \mathrm{sec}^{-3}$, the increases occur for smail and large radius ratios $(\approx 30 \%)$.

For $15 \mu \mathrm{m}$ collector drops, the overall largest increase of the collision efficiencies associated with $\epsilon=1 \mathrm{~cm}^{2} \mathrm{sec}^{-3}$ takes place in the middle range of radius ratios $(\approx 20 \%)$, the largest increase associated with $\epsilon=10 \mathrm{~cm}^{2} \mathrm{sec}^{-3}$ in the lower range of 
radius ratios $(\approx 30 \%)$, and the largest increase associated with $\epsilon=100 \mathrm{~cm}^{2} \mathrm{sec}^{-3}$ in the upper range of radius ratios $(\approx 20 \%)$.

Finally, for $20 \mu \mathrm{m}$ collector drops, some similarities with the collision efficiencies for $15 \mu \mathrm{m}$ collector drops can be found. The region of the increase of the collision efficiency for $\epsilon=1 \mathrm{~cm}^{2} \mathrm{sec}^{-3}$ broadens toward higher ratios of drop radii compared to that for $15 \mu \mathrm{m}$ drops. Again, for $\epsilon=10 \mathrm{~cm}^{2} \mathrm{sec}^{-3}$, the increase is limited to small radius ratios. While, for $\epsilon=100 \mathrm{~cm}^{2} \mathrm{sec}^{-3}$, the highest increases occur for the radius ratios equal 0.4 and 0.8 ; these local maxima being the highest increases observed for $20 \mu \mathrm{n}$ collector drops $(\approx 50 \%)$.

The overall picture of the effect of turbulence on the collision efficiencies is a very complicated, not to say, a very obscure one. In the next section, we will try to comment on some of the results from the point of view of scale analysis.

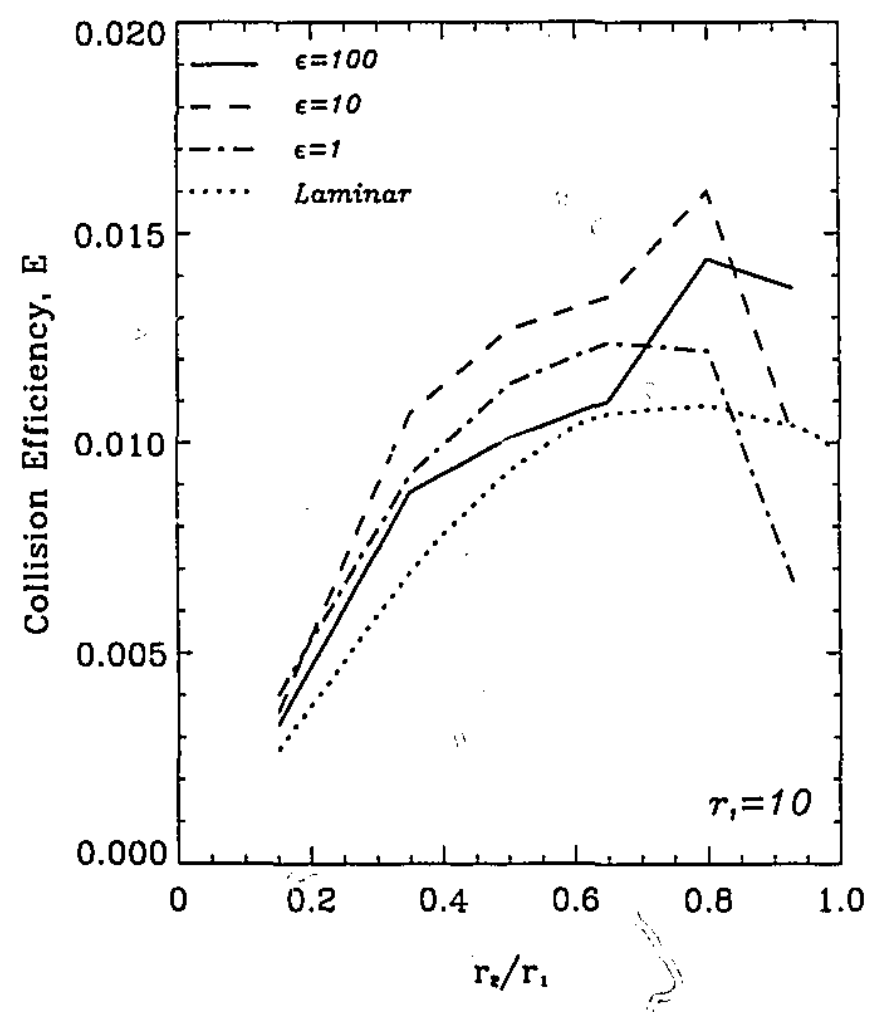

Fig. $\quad 9.2$ Comparison of the collision efficiencies for different strengths of turbulence with the laminar collision efficiencies for $10 \mu \mathrm{m}$ collector drops. 


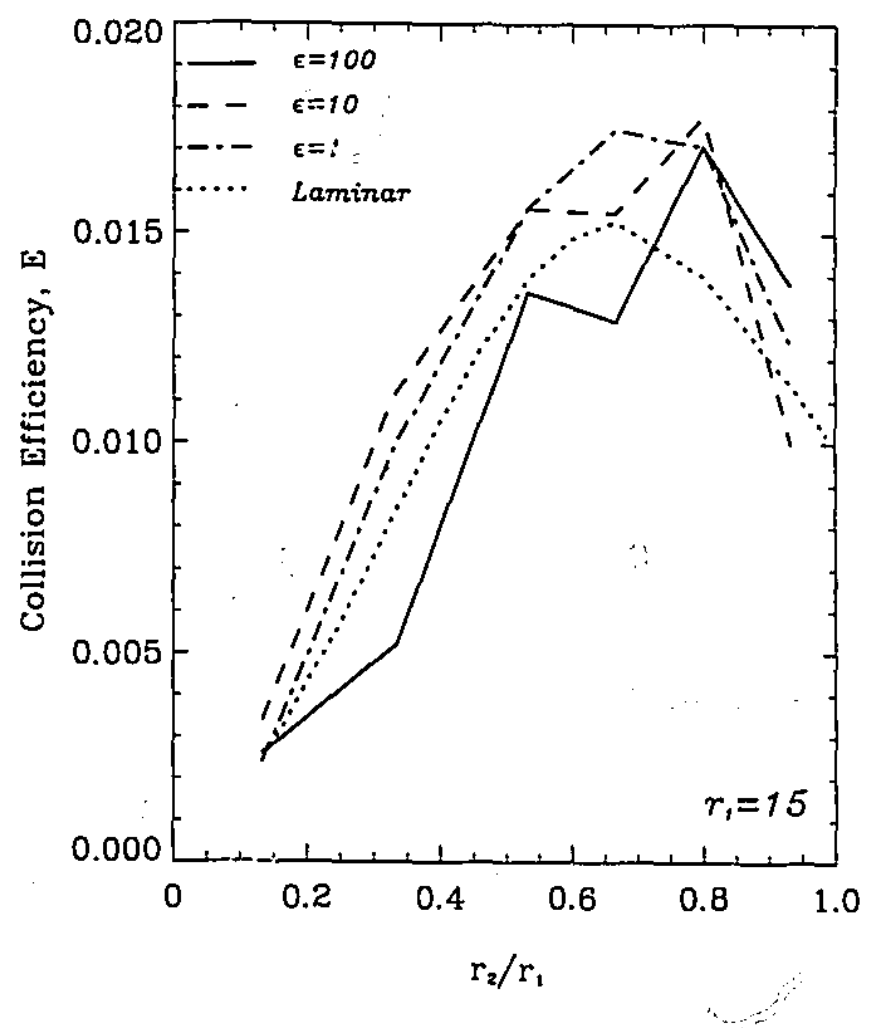

Fig. $\quad 9.3$ Comparison of the collision efficiencies for different strengths of turbulence with the laminar collision efficiencies for $15 \mu \mathrm{m}$ collector drops.

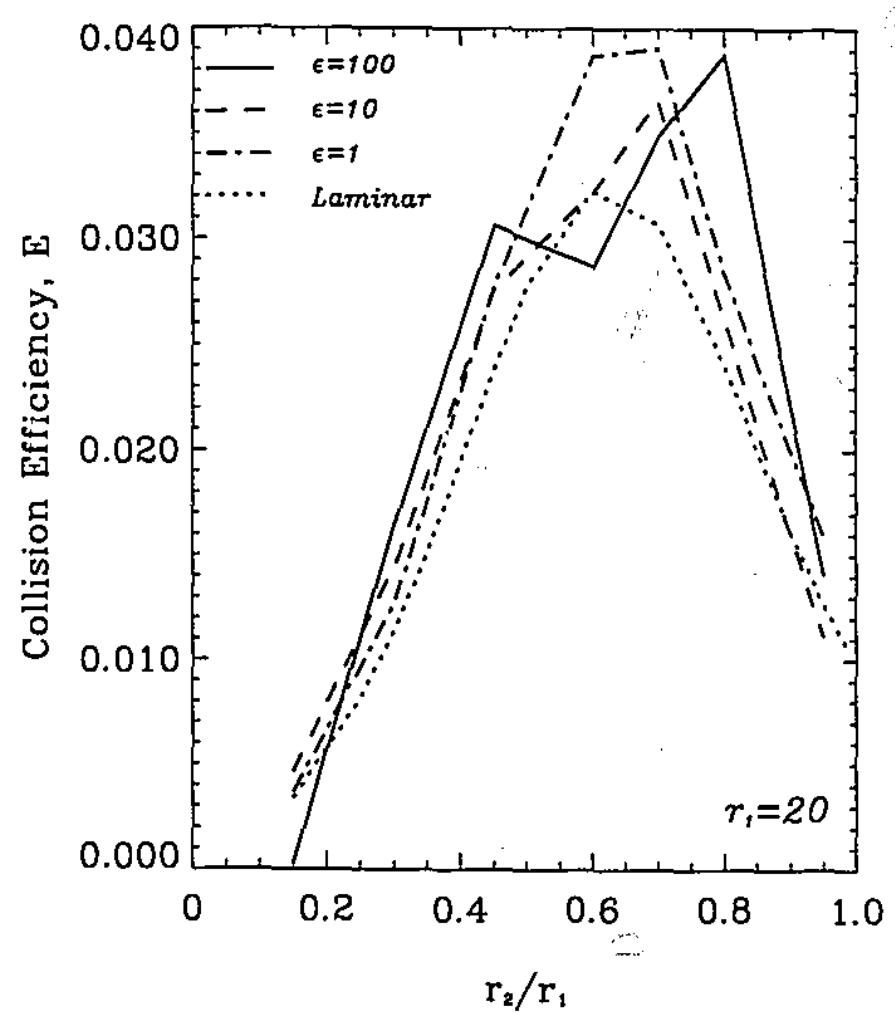

Fig. $\quad 9.4$ Comparison of the collision efficiencies for different strengths of turbulence with the laminar collision efficiencies for $20 \mu \mathrm{m}$ collector drops. 


\subsection{Results from the Point of View of Scale Analysis}

Turbulence which is characterized by multiple scale behaviour should not be expected to interact with drop pairs of different sizes in a similar manner. In Chapter 2 , we introduced scales characterizing both turbulence and the binary drop interactions. We will return to the concepts which were introduced there. In Figs. 9.5-9.7, we plot the length, time, and velocity scales respectively for all three collector drops vs. radius ratio. Also, we mark in the figures the Kolmogorov length, time, and velocity for different strengths of turbulence. Additionally, in Fig. 9.6, not only the Kolmogorov time scales are juxtaposed with the scales characterizing drops but also the relaxation times for all three collector drops. These figures will be crucial in our later arguments concerning behaviour of the collision efficiencies generated by our model.

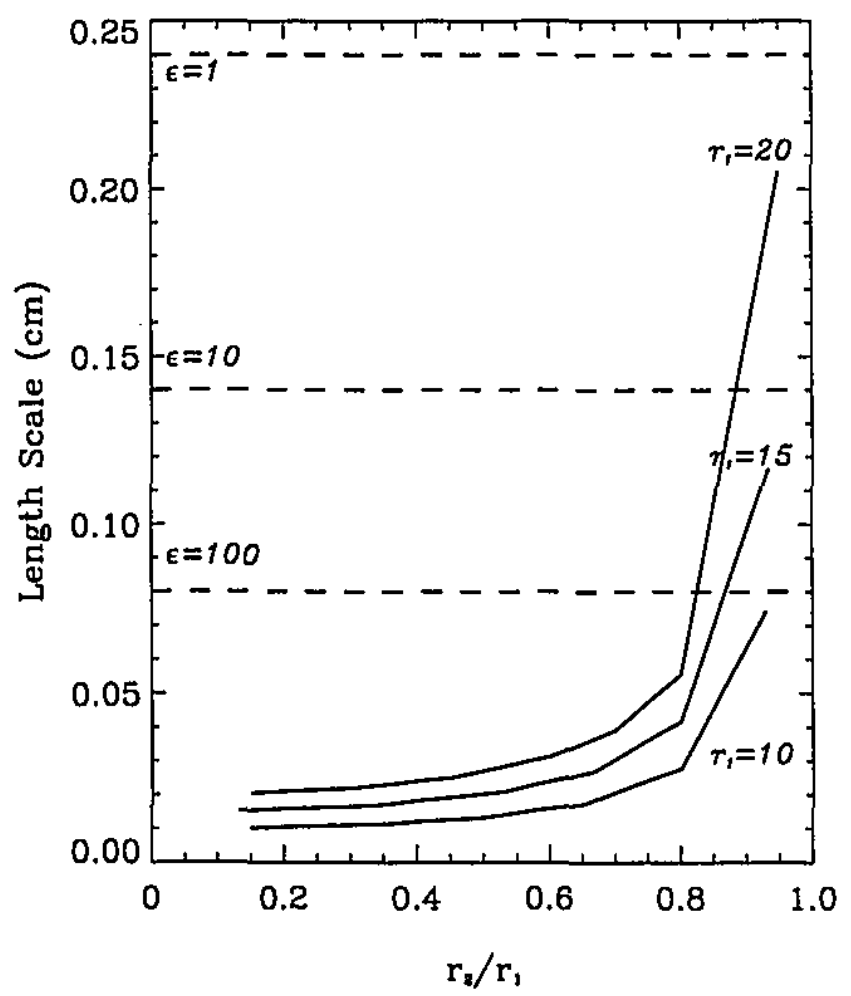

Fig. 9.5 Comparison of the two-drop length scales for different drops (solid lines) with the Kolmogorov length scales for different strengths of turbulence (dashed lines). 

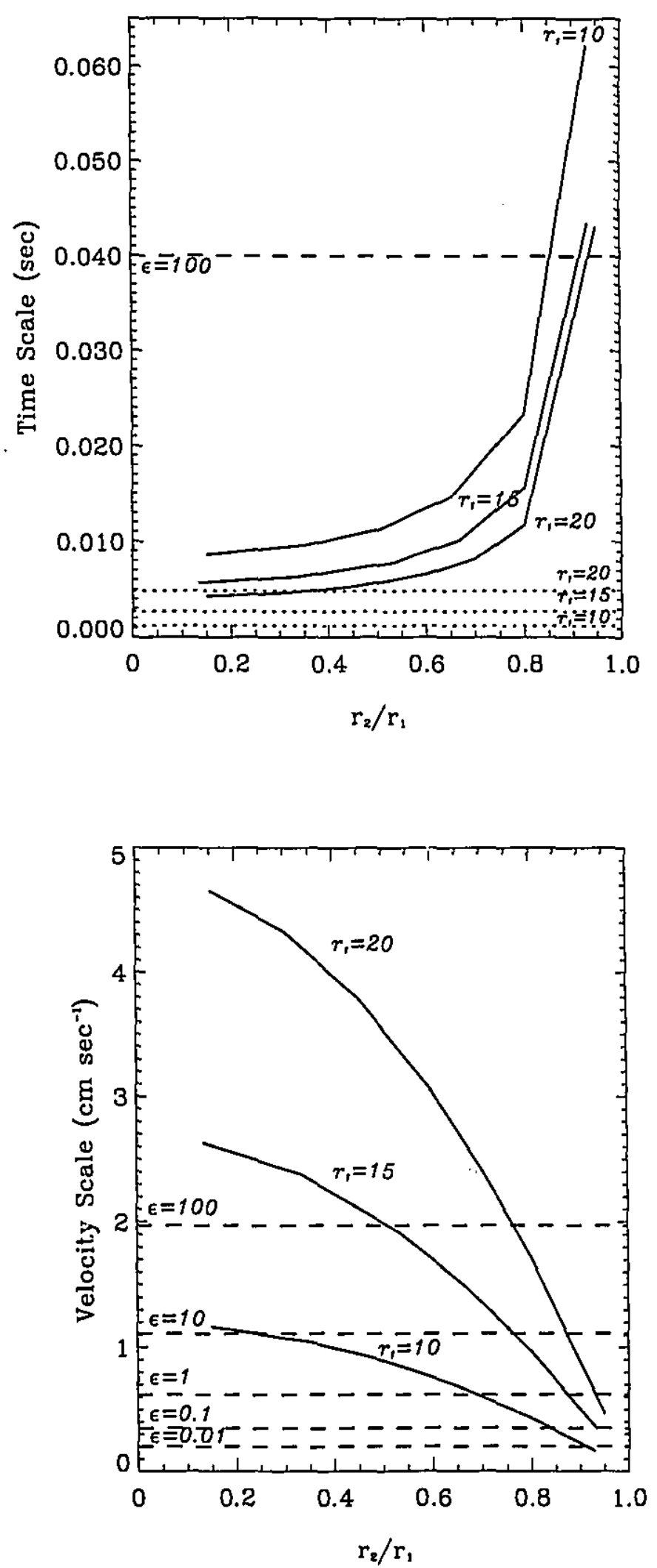

Fig. $\quad 9.6$ Comparison of the two-drop time scales for different drops (solid lines) with the Kolmogorov time scales for different strengths of turbulence (dashed line; values for $\epsilon=1$ and 10 $\mathrm{cm}^{2} \mathrm{sec}^{-3}$ are off-scale and can be found in $\mathrm{Ta}$ ble 2.3), and the relaxation times for different collector drops (dotted lines).
Fig. 9.7 Comparison of the terminal velocity differences for different pairs of drops (solid lines) with the Kolmogorov velocity scales for different strengths of turbulence (dashed lines). 
As it was mentioned before, no simple dependence of collision efficiency on parameters is expected to be established. We may, however, attempt to identify different ways in which turbulence interferes with gravitational settling of droplets. Because the collision efficiency is calculated as an integral of $P(\theta) \sin 2 \theta$, we use this curve to define our criteria. $(P(\theta) \sin 2 \theta$ provides much more information than the collision efficiency itself.)

\subsubsection{Small Pelturbation to Gravitational Laminar Settling}

Let us consider a class of drop pairs for which the curve $P(\theta) \sin 2 \theta$ resembles that for gravitational settling in a still fluid (see Fig. 9.8). Let the abscissa of the maximum of $P(\theta) \sin 2 \theta$ for the turbulent flow be $\theta^{\text {tur }}$, and for the laminar flow $\theta^{\text {lari }}$. We define the cases of small perturbation to the gravitational laminar settling according to the following criterion

$$
\theta^{\text {lam }} \geq 0.8 \theta^{t u r}
$$

The following sets of parameters fit the above criterion:

$r_{1}=10 \mu \mathrm{m}$ and $\epsilon=1 \mathrm{~cm}^{2} \mathrm{sec}^{-3} ; r_{2}=5,6.5 \mu \mathrm{m}$,

$r_{1}=15 \mu \mathrm{m}$ and $\epsilon=1 \mathrm{~cm}^{2} \mathrm{sec}^{-3} ; r_{2}=5,8,10,12 \mu \mathrm{m}$,

$r_{1}=20 \mu \mathrm{m}$ and $\epsilon=1 \mathrm{~cm}^{2} \mathrm{sec}^{-3} ; r_{2}=3,6,9,12,14,16 \mu \mathrm{m}$.

Notice that all drop pairs which we consider in this section are characterized by both length and time scales significantly smaller than the Kolmogorov length and time (respectively 25 and 200 times smaller). Therefore, the effect of turbulent eddies on the relative trajectories of the drops is small. Still, a relatively significant effect on the collision efficiencies can be observed. All the above cases are characterized by a moderate increase of the collision efficiency. Clearly, the majority of droplet pairs for $\epsilon=1 \mathrm{~cm}^{2} \mathrm{sec}^{-3}$ belong to this category. The few remaining cases involving small and 
large radius ratios will be discussed later.
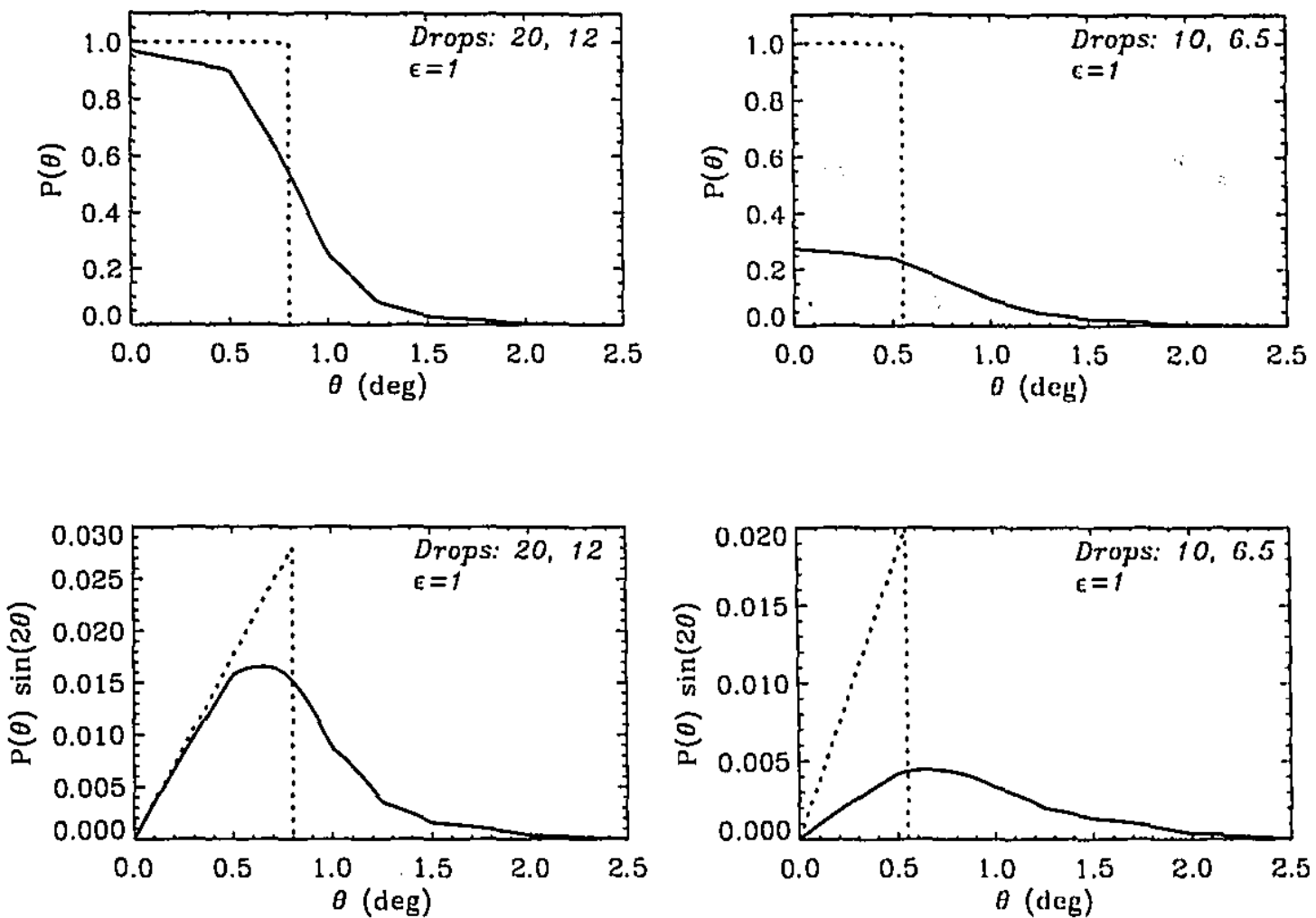

Small perturbation to gravitational laminar settling

Fig. 9.8 Probability $P(\theta)$ and $P(\theta) \sin 2 \theta$ illustrating the small perturbation mechanism: $\left(r_{1}=20 \mu \mathrm{m}, r_{2}=12 \mu \mathrm{m}, \epsilon=1 \mathrm{~cm}^{2} \mathrm{sec}^{-3}\right)$, and $\left(r_{1}=10 \mu \mathrm{m}, r_{2}=6.5\right.$ $\left.\mu \mathrm{m}, \epsilon=1 \mathrm{~cm}^{2} \mathrm{sec}^{-3}\right)$; increase of the collision efficiency. Dotted lines represent the laminar cases.

Let us present more results. These wore obtained for very weak turbulent fields $\left(\epsilon=0.1\right.$ and $\left.0.01 \mathrm{~cm}^{2} \mathrm{sec}^{-3}\right)$, but the initial distance between drops $R$ was, similarly to the cases with strong turbulence, equal to 20 radii of the collector drop. Therefore, the results do not have quantitative value (we underestimate the hydrodynamic interactions). The calculations were performed only. to illustrate the effect of very 
weak turbulence on collision efficiencies. Table 9.2 is analogous to Table 9.1 for weak turbulence, and Figs. 9.9, and 9.10-9.11 are analogous to Figs. 9.1 and 9.2-9.3, except that the laminar values of the collision efficiencies were obtained for the initial distance between drops equal to 20 radii of the collector drop, in order to facilitate comparisons. According to our criterion, all combinations of parameters $\left(\epsilon, r_{1}\right.$, and $r_{2}$ ) yield $P(\theta) \sin 2 \theta$ slightly perturbed compared to the laminar settling.

Characteristically, for very weak turbulence, if the ratio of the Kolmogorov velocity to the drop terminal velocity difference becomes small (e.g. 0.5 for $\epsilon=0.1$ $\mathrm{cm}^{2} \mathrm{sec}^{-3}$, and $r_{1}=10 ; 0.3$ for $\epsilon=0.1 \mathrm{~cm}^{2} \mathrm{sec}^{-3}$, and $r_{1}=15$; etc.), no effect on collision efficiency is found.
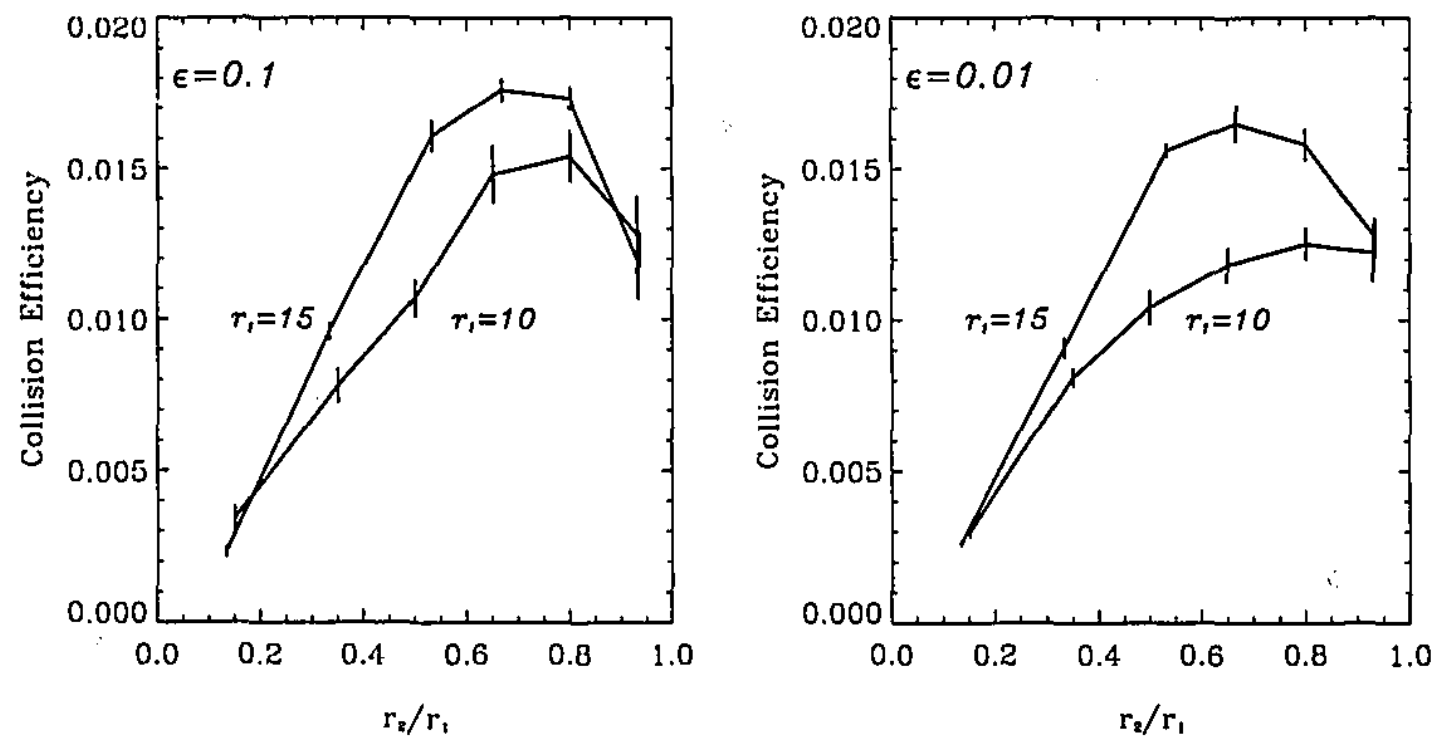

Fig. 9.9 Collision efficiencies for very weak turbulent flows with different rates of energy dissipation $\left(\epsilon=0.1,0.01 \mathrm{~cm}^{2} \mathrm{sec}^{-3}\right)$. 

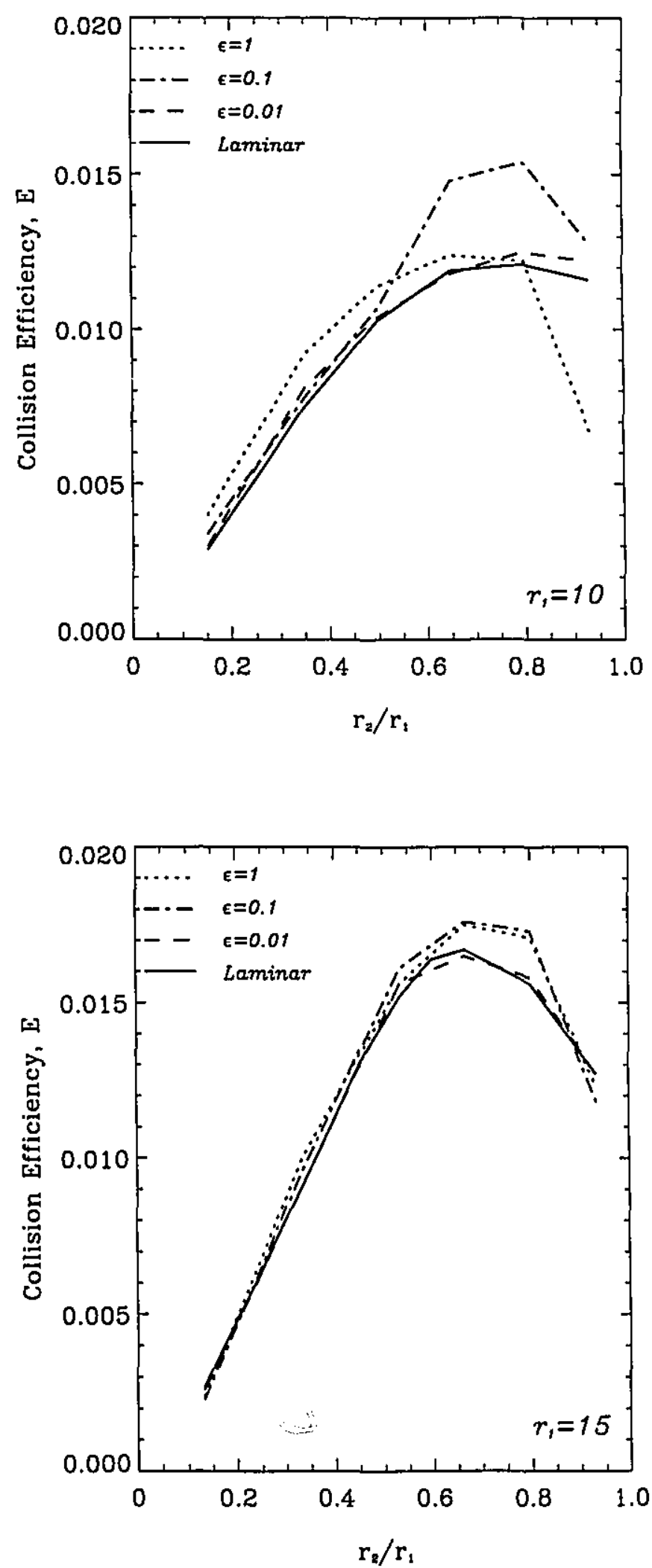

Fig. $\quad 9.10$ Comparison of the collision efficiencies for different strengths of weak turbulence with the laminar collision efficiencies for the $10 \mu \mathrm{m}$ collector drop. (The laminar collision efficiencies were obtained for the same drop separations as those for turbulent, flows.)
Fig. $\quad 9.11$ Comparison of the collision efficiencies for different strengths of weak turbulence with the laminar collision efficiencies for the $15 \mu \mathrm{m}$ collector drop. (The laminar collision effieiencies were obtained for the same drop separations as those for turbulent flows.) 
TABLE 9.2

Turbulent Collision Efficiencies

for Very Weak Turbulence

\begin{tabular}{|c|c|c|c|c|c|c|c|}
\hline $\begin{array}{l}\text { Radius } r_{1} \\
\qquad(\mu \mathrm{m})\end{array}$ & $\begin{array}{l}\text { Radius } r_{2} \\
(\mu \mathrm{m})\end{array}$ & $\left(\mathrm{cm}^{2} \mathrm{sec}^{-3}\right)$ & $\begin{array}{l}\text { Radius } R \\
\quad(\mu \mathrm{m})\end{array}$ & $\begin{array}{l}\text { Truncation } \\
\text { Factor }\end{array}$ & $\begin{array}{l}\text { Collision } \\
\text { Efficiency }\end{array}$ & $\begin{array}{c}\text { Error } \\
(\%)\end{array}$ & $\begin{array}{c}\text { Relative } \\
\text { Change (\%)† }\end{array}$ \\
\hline 10 & 1.5 & 0.01 & 200 & 1000 & 0.0030 & 7 & +3 \\
\hline 10 & 3.5 & 0.01 & 200 & 1000 & 0.0081 & 4 & +8 \\
\hline 10 & 5.0 & 0.01 & 200 & 600 & 0.0104 & 5 & +1 \\
\hline 10 & 6.5 & 0.01 & 200 & .400 & 0.0118 & 5 & -1 \\
\hline 10 & 8.0 & 0.01 & 200 & 400 & 0.0125 & 4 & +3 \\
\hline 10 & 9.3 & 0.01 & 200 & 200 & 0.0122 & 8 & +5 \\
\hline 10 & 1.5 & 0.1 & 200 & 400 & 0.0034 & 11 & +17 \\
\hline 10 & 3.5 & 0.1 & 200 & 200 & 0.0078 & 7 & +4 \\
\hline 10 & 5.0 & 0.1 & 200 & 200 & 0.0107 & 6 & +4 \\
\hline 10 & 6.5 & 0.1 & 200 & 200 & 0.0148 & 7 & +24 \\
\hline 10 & 8.0 & 0.1 & 200 & 200 & 0.0154 & 5 & +27 \\
\hline 10 & 9.3 & 0.1 & 200 & 50 & 0.0128 & 10 & +10 \\
\hline 15 & 2.0 & 0.01 & 300 & 1500 & 0.0026 & 3 & +4 \\
\hline 15 & 5.0 & 0.01 & 300 & 1000 & 0.0091 & 4 & +0 \\
\hline 15 & 8.0 & 0.01 & 300 & 800 & 0.0156 & 1 & +3 \\
\hline 15 & 10.0 & 0.01 & 300 & 800 & 0.0165 & 4 & -1 \\
\hline 15 & 12.0 & 0.01 & 300 & 400 & 0.0158 & 3 & +1 \\
\hline 15 & 14.0 & 0.01 & 300 & 200 & 0.0127 & 5 & +0 \\
\hline 15 & 2.0 & 0.1 & 300 & 400 & 0.0023 & 8 & +16 \\
\hline 15 & 5.0 & 0.1 & 300 & 400 & 0.0096 & 3 & +6 \\
\hline 15 & 8.0 & 0.1 & 300 & 400 & 0.0161 & 3 & +6 \\
\hline 15 & 10.0 & 0.1 & 300 & 200 & 0.0176 & 2 & +5 \\
\hline 15 & 12.0 & 0.1 & 300 & 200 & 0.0173 & 2 & +11 \\
\hline 15 & 14.0 & 0.1 & 300 & 200 & 0.0118 & 9 & -7 \\
\hline
\end{tabular}

With the help of scale analysis, we shed some light on the behaviour of the collision efficiency curves for weak turbulence. The relatively uniform increase of the collision efficiencies for $\epsilon \leq 1 \mathrm{~cm}^{2} \mathrm{sec}^{-3}$ semms to be caused by small perturbation to the gravitational laminar settling. Previously in this chapter, we noticed that for ratios of drop radii greater than 0.9 the values of collision efficiencies for different collector drops vary, and are different from those for laminar flow. It is clear now 
that this behaviour is not caused by small perturbations to the gravitational laminar settling but rather by some other mechanism, possibly turbulent mixing. Finally, the collision efficiencies for small radius ratios and collector drops of radii 10 and $15 \mu \mathrm{m}$, for which (9.1) also does not apply must be affected by some other mechanism.

\subsubsection{Turbulent Mixing}

For stronger turbulence, a significant departure of the $P(\theta) \sin 2 \theta$ curves from the laminar ones can be observed. (See Fig. 9.12.) All examined cases for $\epsilon \geq 10$ belong to this category. No simple conclusions can be derived for stronger turbulence. This is because the motion of drops and the possibility of collisions between them is determined by several factors each of them strongly depending on the drop radius ratio as well as drop masses.

Let us specify these factors. Gravity causes collision while hydrodynamic interactions prevents collisions. For gravitational laminar settling, the highest collision efficiencies occur in the middle range of radius ratios. Turbulent eddies affect, the relative motion the most for large radius ratios-that is probably why for stronger turbulence, the maxima of the collision efficiency curves are shifted toward higher values of radius ratios. Time variations of the turbulent field are also felt by drop pairs with high radius ratios, and also for smaller collector drops which follow the turbulent trajectories more closely (small inertia). Further, there is the effect of inertia which could influence the relative motion of drops with small radius ratios, and larger collector drops. Finally, the hydrodynamic interactions are modified by the presence of a turbulent velocity field (recall the presence of a velcity gradient term in the drop equations of motion). So many are the factors acting to influence (positively and negatively) the process of drop collisions that it is not surprising the

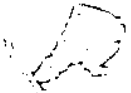


final result-the collision efficiency-is not a simply function of the drop sizes and the strength of turbulence.
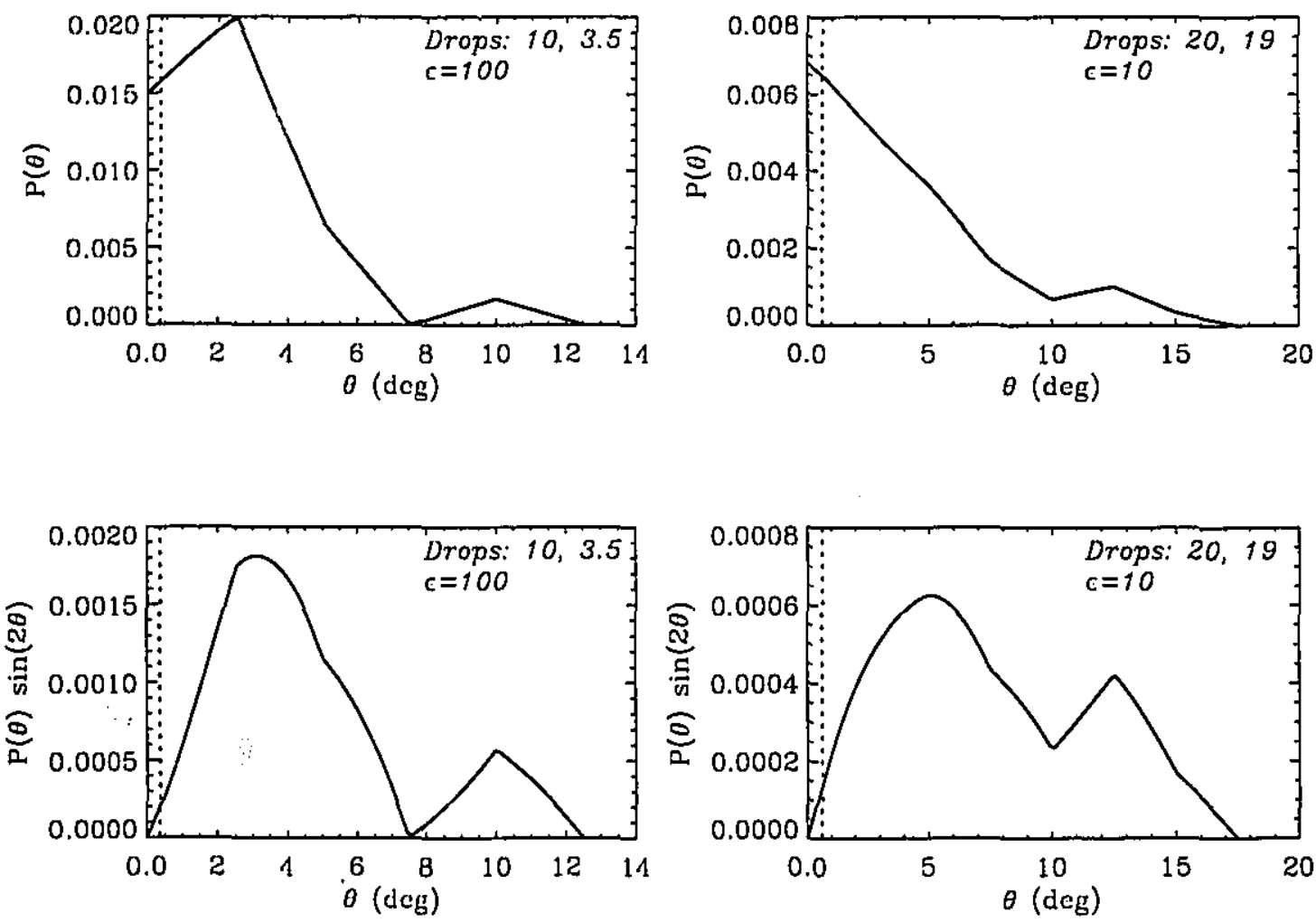

Turbulent mixing

Fig. 9.12 Probability $P(\theta)$ and $P(\theta) \sin 2 \theta$ illustrating turbulent mixing: $\quad\left(r_{1}=10\right.$ $\left.\mu \mathrm{m}, r_{2}=3.5 \mu \mathrm{m}, \epsilon=100 \mathrm{~cm}^{2} \mathrm{sec}^{-3}\right)$, and $\left(r_{1}=20 \mu \mathrm{m}, r_{2}=19 \mu \mathrm{m}, \epsilon=10 \mathrm{~cm}^{2}\right.$ $\left.\sec ^{-3}\right)$. Dotted lines represent angles $\theta$ corresponding to the grazing trajectories.

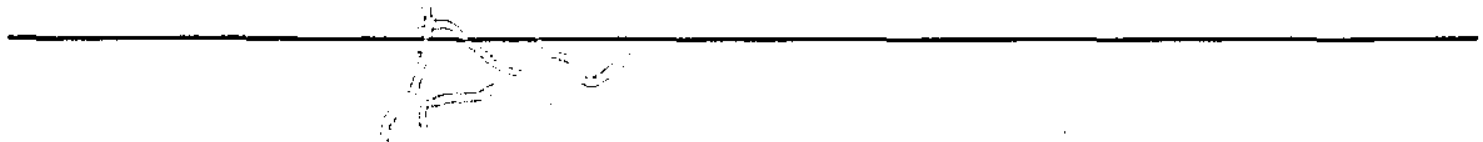




\subsection{Collision Kernels and the Extension of the Re- sults for Identical Drops}

From the point of view of the Stochastic Growth Equation, the collision kernels provide more physical insight than the collision efficiencies. This is because they are proportional to the collision rates. We provide the collision kernels for all three strengths of turbulence $\left(100,10\right.$, and $\left.1 \mathrm{~cm}^{2} \mathrm{sec}^{-3}\right)$, and laminar flow in Fig. 9.13. The tendencies observed for the collision kernels are obviously the same as those for the collision efficiencies. We shall, however, point out a general trend which makes the turbulent collision kernel curves more flat in the middle range of radius ratios in comparison to the laminar collision kernels curves as turbulence becomes more vigorous. In other words, for strong turbulence, the collision kernels tend to be independent of radius ratio in the range of radius ratios from 0.3 to 0.8 .

We want to extend the range of radius ratios in order to account for collisions between almost identical drops. The methodology applied to derive the previously presented results became insufficient for radius ratios approaching one because of the small number of collisions per number of experiments. The number of trajectory calculations which would have had to be carried out in order to obtain useful results was unacceptable large. Therefore, we introduced a shortcut approach to rather estimate the order than to calculate the collision kernels. In our approach, there is a built-in but not quite accurate assumption that the effect of hydrodynamic interactions can be neglected altogether for the range of radius ratios under consideration. In order to obtain the collision kernels, the 'in' component of the relative velocity as well as the fraction of droplets which are going 'in' were calculated at the sphere of radius $r_{1}+r_{2}$. The details of the numerical procedure were described in the section dedicated to the calculation of the 'in' component of the relative drop velocity in Chapter 8 . 

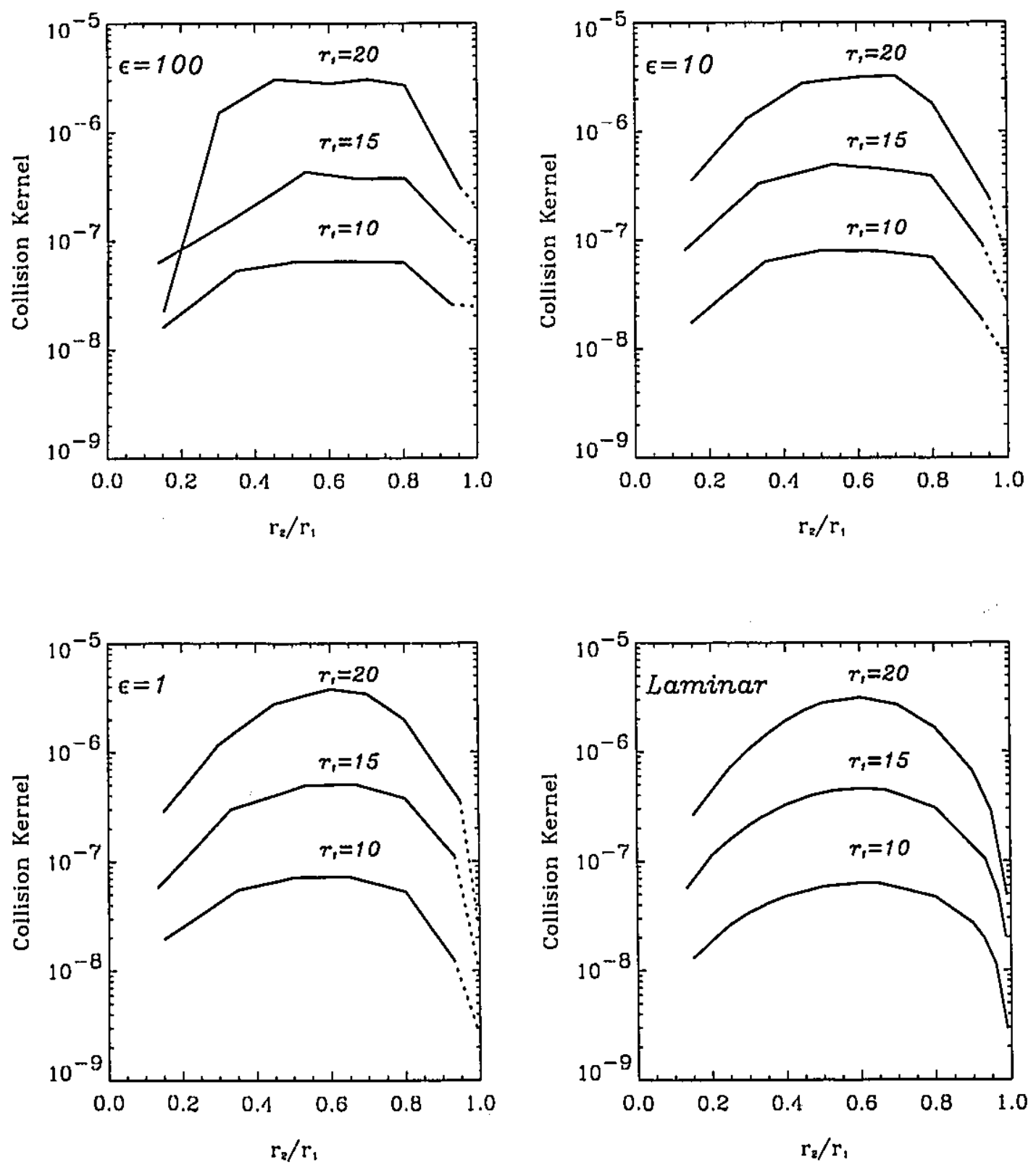

Fig. 9.13 Collision kernels for turbulent flows with different rates of energy dissipation $\left(\epsilon=100,10,1 \mathrm{~cm}^{2} \mathrm{sec}^{-3}\right)$, and for laminar flow. Dotted lines mark the extension of the collision kernels for identical drops. 
The final touch to our calculation was performed in a rather crude way. In Chapter 7 , we found that in the case of two-dimensional deformation field, the ratio of the collision kernel of non-interacting drops to the collision kernel of interncting drops (for $15 \mu \mathrm{m}$ drops) was 9 . We used that number to modify the obtained collision kernels. (These are the underestimates of the collision kernels while the unmodified values are the overestimates.) In Fig. 9.15, the extended values of the collision kernels are marked with dotted lines. The increases, as expected, are monotonically increasing functions of the rate of energy dissipation.

\subsection{Suggestions for the Improvement of the Results}

We divide the possible improvements into two categories: the first includes the treatment of the hydrodynamic interactions and the representation of turbulence; and the second includes some refinements to the procedure of obtaining collision efficiencies and collision kernels. As far as the hydrodynamic interactions are concerned, 1 the major improvements would involve the calculation of the force and torque coefficients with the slip-flow boundary conditions. Further, the turbulent field used in the present research might be substituted by the direct simulation of turbulence. Both changes would require a major modification of the methodology, a rather time consuming one, and, in the case of the direct simulation of turbulence, probably computationally expensive. With the present results suggesting a very moderate influence of turbulence on the process of collisional growth of cloud droplets, to invest in new methods seems to be an extravagant step.

On the other hand, the methods of calculating collision efficiencies and collision kernels have potential for some improvement without major restructuring of the existing model. The most troublesome set of parameters for which we tried to obtain 
collision efficiencies involved strong turbulence and very high values of radius ratios. The scatter of drops due to turbulence did not allow us to obtain satisfactory results. It is worthwhile to point out the two effects which we try to capture while calculating the probability of collision, $P(\theta)$. These are turbulent mixing and hydrodynamic interactions. A skillful and methodologically sound method of separating these two effects may lead to a better way of calculating collision efficiencies for the troublesome parameters. In particular, the probability of collision for interacting drops may be represented by the probability of collision for non-interacting drops modified by a function of $\theta$ representing the average interaction between drops. This funciion can be obtained in an analogous way as the collision efficiency for laminar flows (along the line of thinking presented in Chapter 7\%, and, later, averaged. Presumably, such a separation would allow one to calculate the time consuming statistics using noninteracting drops with significant savings of the computer time.

We also see some relatively simple ways to improve the method of estimating collision kernels for almost equal and equal drops. In the previous section, we obtained the collision kernels as 'in' fluxes of small drops through the sphere of radius equal to the sum of drop radii centred at the position of the collector drop. The relative 'in' velocity distribution which we calculated was a correct one. The problem is that the concentration of small droplets in the vicinity of a large drop differs from the ambient concentration. We think that a simple method to obtain the bulk degree of attenuation of the number of small drops in the vicinity of a collectcr drop as a function of the relative distance between drops can be easily developed with the help of our numerical model. 


\section{Conclusions}

Up until present, the only systematic and comprehensive examination of the influence of turbulence on coliision efficiencies was that by de Almeida $[1975,1976$, and 1979]. The effect of turbulence which de Almeida's drops experienced was dramaticthe increase of collision efficiencies being ten fold and larger. No such dramatic behaviour here. Our model predicts moderate, at maximum 1.5-fold increases; and, for some combinations of drop radii and rate of energy dissipation our model predicts even decreases of the collision efficiency. (In view of our results some of the critics of de Almeida's work were certainly correct.)

Let us summarize our results in a concise way. Weak turbulence $\left(1 \mathrm{~cm}^{2} \mathrm{sec}^{-3}\right)$ causes a moderate and relatively uniform increase of the collision efficiencies for all examined collector drops, except for the cases with small (0.15) and large (0.93) radius ratios. Drops belonging to these categories behave in a different way for different collector drops. In particular, for $r_{2} / r_{1}=0.15$, the increase is significant for the 10 $\mu \mathrm{m}$ collector drop but very small for the $20 \mu \mathrm{m}$ collector drop. The opposite is true for $r_{2} / r_{1}=0.93$, where for the $10 \mu \mathrm{m}$ collector drop the collision efficiency decreases, and for the $20 \mu \mathrm{m}$ collector drop it increases.

For strong turbulence (10 and $\left.100 \mathrm{~cm}^{2} \mathrm{sec}^{-3}\right)$, the behaviour is more complex. Still, some general conclusions can be derived. Persistently, there is a tendency for the turbulent collision efficiency to differ little from the laminar collision efficiency in the range of radius ratios from 0.55 to 0.7 (this range varies for different combinations 
of parameters.) The significant increases occur for both the radius ratios below and above the 'no-change' region. For $\epsilon=10 \mathrm{~cm}^{2} \mathrm{sec}^{-3}$, the preferred range of increases is predominantly below the 'no change' region while for $\epsilon=100 \mathrm{~cm}^{2} \mathrm{sec}^{-3}$ above that region. There are though, some exceptions from the above rules that, as we hypothesized about in Chapter 9, can be attributed to the different mechanisms of interaction between turbulence and drop gravitational settling.

How does the behaviour described above affect the growth of drop spectra? The application of the collision efficiencies that we obtained in the Stochastic Growth Equation was not planned as a part of this thesis. The task may be performed later on. Certainly we do not expect a very pronounced effect because the values of the turbulent collision efficiencies differ relatively little from laminar collision efficiencies. However, there are situations for which some subtle effects may be expected. Perhaps, these might have a distinctive character for different rates of energy dissipation. Our first candidate for an effect is $\epsilon=10 \mathrm{~cm}^{2} \mathrm{sec}^{-3}$. (The highest overall increase of the collision efficiencies for $10 \mu \mathrm{m}$ collector drops were obtained for $\epsilon=10 \mathrm{~cm}^{2} \mathrm{sec}^{-3}$.) The effect of the increase of collision efficiencies due to turbulence for such small collector drops on the drop spectra has not been examined. The effect of $\epsilon=100$ $\mathrm{cm}^{2} \mathrm{sec}^{-3}$, because of the peculiar forms of the collision efficiency curves, may also yield interesting results. On the other hand, the effect of weak turbulence will be the least interesting because the calculated collision efficiencies are roughly proportional to the laminar collision efficiencies, and the maximum relative increases are generally somewhat smaller than those for stronger turbulence.

Finally, let us quote Aristotle (384-322 B.C.): "Dear is Plato, but dearer still the truth". Whoever attempted to resolve the issue of the effect of turbulence on collision rates before, did it probably with a hope that the effect is important. So, did we. The answer, however, is no, and must be accepted with equal enthusiasm. 


\section{Bibliography}

Ackerman, B., 1967: The nature of meteorological fluctuation in clouds. J. Appl. Meteor., 6, 61-71.

AlmeidA DE, F. C., 1975: On the Effect of Turbulent Fluid Motion in the Collisional Growth of Aerosol Particles. Ph.D. Thesis, University of Wiscinsin, Madison, $185 \mathrm{pr}$.

AlmeidA DE, F. C., 1976: The collision problem in cloud droplets moving in a turbulent environment-Part I: A method of solution. J. Atmos. Sci., 33, 1571-1878.

AtsmeIDA DE, F. C., 1979: The collisional problem of cloud droplets moving in a turbulent environment-Part II: Turbulent collision efficiencies. J. Atmos. Sci., 36, 1564-1576.

ARENBERG, D., 1939: Turbulence as a major factor in the growth of cloud droplets. Bull. Amer. Met. Soc, 20, 444-445.

Baker, M. B., R. E. Breidental, T. W. Choularton, and J. Latham, 1984: The effect of turbulent mixing in clouds. J. Atmos. Sci., 41, 1123-1138.

BAKER, M. B., AND J. LATHAM, 1982: A diffusive model of the turbulent mixing of dry and cloudy air. Quart. J. Roy. Meteor. Soc., 108, 871-898.

Batchelor, G. K., 1953: The Theory of Homogeneous Turbulence. Cambridge University Press, Cambridge, $197 \mathrm{pp}$.

BeARD, K. V., AND H. R. PRUP.FACHER, 1969: A determination of the terminal velocity and drag of small water drops be means of a wind tunnel. J. Atmos. Sci., 26, 1066-1072.

BöHM, J. P., 1992: A generallhydrodynamic theory for mixed-phase microphysics. Part II: collision hornels for coalescence. Atmos. Res., 27, 275-290.

Brown, G. P., A. Di Nardo, G. K. Cheng, and T. K. Sherwood, 1946: The flow of gases in pipes at low pressures. J. Appl. Phys., 17, 802-813.

Carrier, G. F., 1953: On slow viscous flow. Final Rept., Brown University, Contract No-653(00), $31 \mathrm{pp}$.

Chapman, S., AND T. G. Cowling, 1970: The Mathematical Theory of Nonuniform Gases. 3rd ed., Cambridge at the University Press, 423 pp. 
Chin, E. H. C., ANd M. Neiburger, 1972: A numerical simulation of the gravitational coagulation process for cloud droplets. J. Atmos. Sci., 29, 718-727.

Comte-Bellot, G., ANd S. ĆORRSin, 1971: Simple Eulerian time correlation of full- and Narrow-band signals in grid-generated, 'isotropic' turbulence. $J$. Fluid Mech., 48, part 2, 273-337.

CoOley, M. D. L., AND M. E. O'Neill, 1969: On the slow motion generated in a viscous fluid by the approach of a sphere to a plane wall or stationary sphere. Mathematika, 16, 37-49.

CoOper, W. A., AND D. BAumgardner, 1988: Comment on "The collection kernel of two falling cloud drops subjected to random perturbations in a turbulent air flow: A stochastic model". J. Atmos. Sci., 46, 1165-1167.

Corrsin, S., 1963: Estimates of the relations between Eulerian and Lagrangian scales in large Reynolds number turbulence. J. Atmos. Sci., 20, 115-119.

Corrsin, S., AND J. LUMLEY, 1956: On the equations of motion for a particle in turbulent fluid. Appl. Sci. Res., A, 6, 114:

Cramír, H., 1940: On the theory of stationary random processes: Ann. Math., 41, 215-230.

DAvis, M. H., 1969: The slow translation and rotation of two unequal spheres in a viscous fluid. Chem. Eng. Sci., 24, 1769-1776.

DAvis, M. H., 1972: Collisions of small cloud droplets: Gas kinetic effects. J. Atmos. Sci., 29, 911-915.

Davis, M. H., AND J. D. SARTOR, 1967: Theoretical collision efficiencies for small cloud droplets in Stokes flow. Nature, 215, 1371-1372.

DEAN, W. R., AND M. E. O'NEILL, 1963: A slow motion of viscous liquid caused be the rotation of a solid body. Mathematika, 10, 13-24.

EAST, T. W. R., AND J. S. MARShaLL, 1954: Turbulence in clouds as a factor in precipitation. Quart. J. R. Met. Soc., 80, 26-47.

FrISCH, H. L., 1954: Steady-state diffusion into a streaming sphere at low Reynolds number. J. Chem. Phys., 20, No. 1, 123-125.

Fuchs, N. A., 1964: The Mechanics of Aeroscis.: Pergamon Press, New York, 398 pp.

Fung, J. C. H., J. C. R. Hunt, N. A. Malik, and R. J. Perkins, 1992: Kinematic simulation of homogeneous turbulence by unsteady random Fourier modes. J. Fluid Mech., 236, 281-318.

GABILLY, A., 1949: On the role that turbulence can play in the coalescence of cloud droplets. Ann. Geophys., 5, 232-234.

Ganatos, P., R. Pfeffer, And S. Weinbaum, 1978: A numerical-solution technique for three-dimensional Stokes flows, with application to the motion of strongly interacting spheres in a plane. J. Fluid Mech., 84, part 1. 79-111. 
Gargett, A. E., T. R. OSBORN, AND P. W. NASMYTH, 1981: Local isotropy and the decay of turbulence in a stratified fluid. J. Fluid Mech., 144, 231-280.

Gibson, M. M., 1963: Spectra of turbulence in a round jet. J. Fluid Mech., 15, 161-173.

Gillespie, D. T., 1972: The stochastic coalescence model for cliud droplet growth. J. Atmos. Sci., 29, 1496-1510.

Gillespie, D. T., 1975: Three models of the coalescence growth of cloud droplets. J. Atmos. Sci., 32, 600-607.

Goldstein, S., 1929: The forces on a solid body moving through viscous fluid. Proc. Roy. Soc. London, A123, 216-235.

Grant, H. L., R. W. Stewart, AND A. Mollliett, 1962: Turbulence spectra from a tidal channel. J. Fluid Mech., 12, 241-263.

HALL, W. D., 1980: A detailed microphysical model within a two-dimensional dynamic framework: Model desiription and preliminary results. J. Atmos. Sci., 37, 2486-2507.

HAPPEL J., AND H. BRENNER, 1965: Low Reynolds Number Hydrodynamics with Special Applications to Particulate Media. Prentice-Hall, Englewood Clifts, N.J., 553 pp.

Heisenberg, W., 1948: On the theory of statistical and isotropic turbulence. Proc. R. Soc. Lond. A, 195, No. 1042, 402-406.

Hinze, J. O., 1975: Turbulence. 2nd ed., McGraw-Hill, New York, 790pp.

Hocking, L. M., AND P. R. Jonas, 1970: The collision efficiency of small drops. Quart. J. R. Met. Soc., 96, 722-729.

INOUE, E., 1951: On the Lagrangian correlation coefficient for the turbulent diffusion and its application to the atmospheric diffusion phenomena. Unpublished report, Geophys. Inst., Univ. of Tokyo.

JEFFREY, D. J., 1982: Low Reynolds number flow between converging splieres. Mathematika, 29, 58-66.

JEFFREY, D. J., 1989: Stresslet resistance functions for low Reynolds number flow using deforming spheres. J. Appl. Math. Phys. (ZAMP), 40, 163-171.

JEFFREY, D. J., 1991: The lubrication analysis for two spheres in a two-dimensional pure-straining motion. Phys. Fluids A, 3, No. 7, 1819-1821.

JEFFREY, D. J., 1992: The calculation of the low Reynolds number resistance functions for two unequal spheres. Phys. Fluids A, 4, No. 1, 16-29.

JEFFREY, D. J., AND Y. ONISHI, 1984: Calculation of the resistance and mobility functions for two unequal rigid spheres in low Reynolds number flow. J. Fluid Mech., 130, 261-290.

JENSEN, J. B., AND BAKER, 1989: The effect of turbulent mixing in clouds. $J$. Atmos. Sci., 41, 2812-2829. 
JENSon, V. G., 1959: Viscous flow around a sphere at low Reynolds numbers (Re $\leq 40)$. Proc. Roy. Soc. London, A249, 346-366.

JoNAS, P. R., 1972: The collision efficiency of small drops. Quart. J. Roy. Met. Soc., 98, 681-683.

JoN AS, P. R., AND P. Goldsmith, 1972: The collection efficiencies of small droplets falling through a sheared air flow. J. Fluid Mech., 52, 593-608.

KERR, R. M., 1990: Velocity, scaír and transfer spectra in numerical turbulence. J. Fluid Mech., 211, 309-232.

K.IM, S., AND S. J. KARRILA, 1991: Microhydrodynamics: Principles and Selected Applications. Butterworth-Heinemann, Boston, $507 \mathrm{pp}$.

Leighton H. G., 1977: Collision efficiency and cloud spectrum evolution. J. Atmos. Sci., 34, 2005-2006.

Kobzunenko, A. G., AND A. I. Neizvestnyy, 1981: Experimental determination of the gravitational coagulation coefficient of water droplets for small Reynolds number. Izv. Atmos. Oceanic Phys., 16, no. 7, 544-547.

Kolmogorov, A. N., 1941: The local structure of turbulence in incompressible viscous fluid for very large Reynolds number. Dokl. Akad. Nauk SSSR, 30, no. 4, 299-303. (Also: 1991, Proc. R. Soc. Lond. A, 434, 9-13.)

Kovasznay, L. S. G., 1948: Spectrum of locally isotropic turbulence. J. Aeronaut. Sci., 16 no. $12,745-753$.

Kraichnan, R. H., 1970: Diffusion by random velocity field. Phys. Fluids, 13, no. $1,22-31$.

Kranogorskaya, N. V., AND A. I. NeizVestnyy, 1973: Experimental studies of the coagulation of charged and neutral droplets of comparable sizes. Meteorologiya $i$ Gidrologiya, 1, 43-47.

LAMB, H., 1932: Hydrodynamics. 6th ed., Dover, New York, 738 pp.

LANDAU, L. D., AND E. M. Lifshitz, 1959: Fluid Mechanics. Pergamon Press, London, 536 pp.

LANGMUIR, I., 1948: The production of rain by a chain reaction in cumulus clouds at temperature above freezing. J. of Meteorol., 5, 175-192.

Lesieur, M., 1990: Turbulence in Fluids. 2nd ed., Kluwer Academic Publishers, Dodrecht, $412 \mathrm{pp}$.

LesliE, D. C., 1983: Developments in the Theory of Turbulence. Claredon Press, Oxford, $367 \mathrm{pp}$.

LEVIN, L. M., AND Y. S. SEDunOV, 1966: Gravitational coagulation of charged cloud drops. Pure Appl. Geophys., 64, 185-196.

LIN, C. L., AND S. C. LEE, 1973: Transient state analysis of separated flow around a sphere. Intern. J. Comp. Fluids, 1,235-250. 
LiN, C. L., AND S. C. LEE, 1975: Collision efficiency of water drops in the atmosphere. J. Atmos. Sci., 32, 1412-1418.

MacPherson, 1979: A Comparison of the Turbulent Characteristics of Cumulus Clouds Measured near Yellowknife and Thunder Bay. Reprint of article from DME/NAE Quarterly Bulletin No. 1979(1), Ottawa, 20 pp.

MACPherson, J. I., AND G. A. IsAAC, 1977: Turbulent characteristics of some Canadian cumulus clouds. J. Appl. Meteor., 16, 81-90.

Manley, R. ST. J., AND S. G. MASON, 1952: Particle motion in sheared suspensions. II. Collisions of uniform spheres. J. Coll. Sci., 7, 355-369.

Mason, B. J., 1971: The Physics of Clouds. Claredon Press, Oxford, $671 \mathrm{pp}$.

Maude, A. D., 1961: End effects in falling-sphere viscometer. Brit. J. Appl. Plyys., 12, 293-295.

MAXEY, M. B., 1987: The gravitational settling of aerosol particles in homogeneous turbulence and random flow fields. J. Fluid Mech., 174, 441-465.

MAXEY, M. B., AND J. J. RILEY, 1983: Equation of motion for a small rigid sphere in a nonuniform flow. Phys. Fluids, 26, 883-889.

Monin, A. S., ANd A. M. Yaglom, 1975: Statistical Fluid Mechanics: Mechanics of Turbulence. Vol. 2, The MIT Press, Cambridge, Massachusetts, $874 \mathrm{pp}$.

NARAshima, R., 1990: Wither Turbulence? Turbulence at the Crossroads. J. L. Lumley, ed., Springer-Verlag, Berlin, 13-48.

NELKIN, M., 1992: In what sense is turbulence an unsolved problem?. Science, 255, $566-570$.

Oвuкноv, A. M., 1941: Spectral energy distribution in a turbulent flow. Dokl. Akad. Nauk SSSR, 32, No. 1, 22-24.

Obukнov, A. M., 1949a: Structure of the temperature field in a turbulent flow. Izv. Akad. Nauk SSSR, Ser. Geogr. i Geofiz., 13, No. 1, 58-69.

Obukнov, A. M., 1949b: Pressure fluctuation in a turbulent flow. Dokl. Akad. Nauk SSSR, 66, No. 1, 17-20.

OCHS III, H. T., R. R. CZYS, AND K. V. BEARD, 1986: Laboratory measurements of coalescence efficiencies for small precipitation drops. J. Atmos. Sci., 43, 225232.

O'NEILL, M. E., 1964: A slow motion of viscous liquid caused by a slowly moving solid sphere. Mathematika, 11, 67-74.

O'Neill, M. E., AND S. R. MAJUmDar, 1970a: Asymmetrical slow viscous fluid motions caused by the translation or rotation of two spheres. Part I: The determination of exact solutions for any values of the ratio of radii and separation parameters. Z. Angew. Math. Phys., 21, 164-179. 
O'Neill, M. E., AND S. R. Majumdar, 1970b: Asymmetrical slow viscous fluid motions caused by the translation or rotation of two spheres. Part II: Asymptotic forms of the solutions when the minimum clearance the spheres approaches zero. Z. Angew. Math. Phys., 21, 180-187.

O'Neill, M. E., AND K. Stewartson, 1967: On the slow motion of a sphere parallel to a nearby plane wall. J. Fluid Mech., 27, part 4, 705-724.

Orszag, S. A., AND G. S. PATterson, 1972: Numerical simulation of turbulence. Statistical Models and Turbulence. ed. M. Rosenblatt, and C. Van Atta, Berlin, Springer-Verlag, 127-147.

Oseen, C. W., 1910: Über die Stokessche Formel und über die verwandte Aufgabe in der Hydrodynamik. Ark. Math. Astron. Fys., 6, No. 29.

Panchev, S., 1971: Random Functions and Turbulence. Pergamon Press, Oxford, $444 \mathrm{pp}$.

PAO, Y.-H., 1965: Structure of turbulent velocity and scalar fields at large wavenumbers. Phys. Fluids, 8, No. 6, 1063-1075.

Pearcey, T., AND G. W. Hill, 1956: A theoretical estimate of the collection efficiencies of small droplets. Quart. J. Roy. Meteor. Soc., 83, 77-92.

Perry, J., 1950: Chemical Engineers' Handbook. 3rd ed., McGraw-Hill, New York, p. 1017.

Picknett, R. G., 1960: Collection Efficiency for water drops in air. Int. J. Air Pollut., 3, 160-167.

Praskovsky, A. A., E. B. Gledzer, M. Yu. Karyakin, and Y. Zhou, 1993: The sweeping decorrelation hypothesis and energy-inertial scale interaction in high Reynolds number flows. J. Fluid Mech., 248, 493-511.

PruppaCher, H. R., AND K. V. BEARD, 1970: A wind tunnel investigation of the internal circulation and shape of water drops falling at terminal velocity in air. Quart. J. Roy. Meteor. Soc., 96, 247-256.

Pruppacher, H. R., AND J. D. KLetT, 1980: Microphysics of Clouds and Precipitation. D. Reidel Publishing Company, Dodrecht, 714 pp.

Reuter, G. W., R. DE Villiers, AND Y. YAViN, 1988: The collection kernel for two falling cloud drops subjected to random perturbations in a turbulent air flow: A stochastic model. J. Atmos. Sci., 45, 765-773.

REYNolDs, O., 1876: Formation of raindrops and hailstones. Nature, 15, 163-165.

Riley, J. J., AND G. S. PATterson, 1974: Diffusion experiments with numerically integrated integrated isotropic turbulence. Phys. Fluids, 17, No. 2, 292-297.

Rogers, R. R., AND M. K. YAU, 1989: Cloud Physics. 3rd ed., Pergamon Press, Oxford, $293 \mathrm{pp}$.

Saffman, P. G., AND J. S. TURNer, 1956: On the collision of drops in turbulent clouds. J. Fluid Mech., 1, 16-30. 
Schlichting, H., 1968: Boundary-Layer Theory. 6rd ed., McGraw Hill, New York, 747 pp.

Shafrir, U., AND M. NEIBURger, 1965: Collision Efficiencies of two spheres falling in a viscous medium. J. Geophys. Res., 68, 4141-4147.

Shampine, L. F., AND M. K. Gordon, 1975: Computer Solution of Ordinary Differential Equations. The Initial Value Problem. W. H. Freeman and Company, San Francisco, 444 pp.

Smirnov, V. I., 1968: Coagulation coefficients of cloud drops (collective effect of different factors). Proc. Intern. Conf. Cloud Physics, Toronto, 91-97.

SMoluchowsKI, M., 1911: On the mutual action of spheres which move in a viscous liquid. Bull. Acad. Sci. Cracovie A, 1, 28-39.

Smoluchowski, M., 1916: Drei Vorträge über Diffusion Brownsche Molecularbewegung und Koagulation von Kolloidteilchen. Physik. Z., 17, 557-571.

SMoluchowsKi, M., 1917: Versuch einer mathematischen Theorie der Koagulationskinetik kolloider Lösungen. Z. Phys. Chem., 92, 9-168.

Stimson M., AND G. B. JefFERY, 1926: The motion of two spheres in viscous fluid. Proc. Roy. Soc, A111, 110-116.

TAYlor, G. I., 1920: Diffusion by continuous movements. Proc. London Math. Soc., Ser. 2, 20, 196-211.

Taylor, G. I., 1935: Statistical theory of turbulence. Proc. Roy. Soc. , A151, $421-478$.

TChen, C. M., 1947: Mean Value and Correlation Problems Connected with the Small Particles Suspended in a Turbulent Fluid. Ph.D. Thesis, Delft., Martinus Nijhoff, The Hague.

TELford, J. W., 1955: A new aspect of coalescence theory. J. Meteor, 12, 436-444.

TENNEKES, H., 1975: Eulerian and Lagrangian time microscales in isotropic turbulence. J. Fluid Mech., 67, part 3, 561-567.

Tennekes, - H., AND J. L. LUMLey, 1972: A First Course in Turbulence. The MIT Press, Cambridge, Massachusetts, $300 \mathrm{pp}$.

TENNEKES, H., AND J. D. Woods, 1973: Coalescence in a weakly turbulent cloud. Quart. J. R. Met. Soc., 99, 758-763.

WAKIYA, S., 1967: Slow motions of a viscous fluid around two spheres. J. Phys. Soc. Japan, 22, 1101-1109.

Woods, J. D., J. C. DRAKe, AND P. Goldsmith, 1972: Coalescence in turbulent cloud. Quart. J. R. Met. Soc., 98, 135-149.

WOODS, J. D., AND B. J. MASON, 1964: Experimental determination of collision efficiencies for small water droplets in air. Quart. J. R. Met. Soc., 90, 373-381.

Yudine, M. I., 1959: Physical consideration of heavy-particle diffusion. Advances in Geophysics, 6, 185-191. 